\title{
Neurocognition and behaviour
}

Citation for published version (APA):

Hellebrekers, D. M. J. (2021). Neurocognition and behaviour: diagnostic work-up and interventions in Duchenne and Becker muscular dystrophy. [Doctoral Thesis, Maastricht University]. Maastricht University. https://doi.org/10.26481/dis.20210423dh

Document status and date:

Published: 01/01/2021

DOI:

10.26481/dis.20210423dh

Document Version:

Publisher's PDF, also known as Version of record

\section{Please check the document version of this publication:}

- A submitted manuscript is the version of the article upon submission and before peer-review. There can be important differences between the submitted version and the official published version of record.

People interested in the research are advised to contact the author for the final version of the publication, or visit the DOI to the publisher's website.

- The final author version and the galley proof are versions of the publication after peer review.

- The final published version features the final layout of the paper including the volume, issue and page numbers.

Link to publication

\footnotetext{
General rights rights.

- You may freely distribute the URL identifying the publication in the public portal. please follow below link for the End User Agreement:

www.umlib.nl/taverne-license

Take down policy

If you believe that this document breaches copyright please contact us at:

repository@maastrichtuniversity.nl

providing details and we will investigate your claim.
}

Copyright and moral rights for the publications made accessible in the public portal are retained by the authors and/or other copyright owners and it is a condition of accessing publications that users recognise and abide by the legal requirements associated with these

- Users may download and print one copy of any publication from the public portal for the purpose of private study or research.

- You may not further distribute the material or use it for any profit-making activity or commercial gain

If the publication is distributed under the terms of Article $25 \mathrm{fa}$ of the Dutch Copyright Act, indicated by the "Taverne" license above, 


\section{Neurocognition and behaviour:}

diagnostic work-up and interventions

in

Duchenne and Becker muscular dystrophy 
Copyright (C) Danique Hellebrekers 2021

All rights reserved. No part of this publication may be reproduced or transmitted in any form or by means, electronical or mechanical, including photocopy, recording or any information storage or retrieval system, without written permission from the author or, when applicable, by the publisher from the published articles.

Cover design: Kimberly R. Buzbulas en tekening door Jeanot S.J. Hellebrekers Lay-out: Tiny Wouters

Printed by: Drukkerij Walters

ISBN: 978-94-6423-187-8 


\title{
Neurocognition and behaviour:
}

\author{
diagnostic work-up and interventions
}

in

\section{Duchenne and Becker muscular dystrophy}

\author{
PROEFSCHRIFT
}

ter verkrijging van de graad van doctor aan de Universiteit Maastricht, op gezag van de Rector Magnificus, Prof. dr. Rianne M. Letschert, volgens het besluit van het College van Decanen, in het openbaar te verdedigen op vrijdag 23 april 2021 om 12.00 uur

door

Danique Mariëtte Josephine Hellebrekers

Geboren op 07 oktober 1991 te Maastricht 


\section{Promotoren}

Prof. dr. J.S.H. Vles (1e promoter)

Dr. J.G.M. Hendriksen (2e promoter, UHD)

\section{Copromoter}

Dr. S. Klinkenberg

\section{Beoordelingscommissie}

Prof. dr. R.J. van Oostenbrugge (voorzitter)

Prof. dr. N. Goemans (Universitair Ziekenhuis Leuven)

Prof. dr. R.W.H.M. Ponds

Prof. dr. C.T.M.R. Stumpel

Prof. dr. J.T. Swaab (Universiteit Leiden) 


\section{Contents}

$\begin{array}{lll}\text { Chapter } 1 & \text { General introduction } & 7\end{array}$

Chapter 2 Cognitive and behavioural functioning in two neurogenetic disorders; how different are these aspects in Duchenne muscular dystrophy and Neurofibromatosis type 1 ? Submitted Journal of the International Neuropsychological Society

Chapter 3 Longitudinal follow-up of verbal span and processing speed in Duchenne muscular dystrophy European Journal of Paediatric Neurology 2020

Chapter $4 \quad$ Minor cognitive impairments in adult males with Becker muscular dystrophy

Chapter 5 The neurocognitive and behavioural profiles of three brothers with Becker muscular dystrophy Child Neurology open 2020

Chapter 6 Instruments for the assessment of behavioural and psychosocial functioning in Duchenne and Becker muscular dystrophy; a systematic review of the literature Journal of Paediatric Psychology 2019

Chapter 7 Computerized working memory training in males with Duchenne muscular dystrophy: a single case experimental design study.

Revisions Neuropsychological Rehabilitation

Chapter 8 Methylphenidate use in males with Duchenne muscular dystrophy and a comorbid attention-deficit hyperactivity disorder European Journal of Paediatric Neurology 2019

Chapter 9 General discussion

\section{Addendum Summary}

Samenvatting

Impact paragraph

Dankwoord

Curriculum vitae 199

List of publications and presentations 


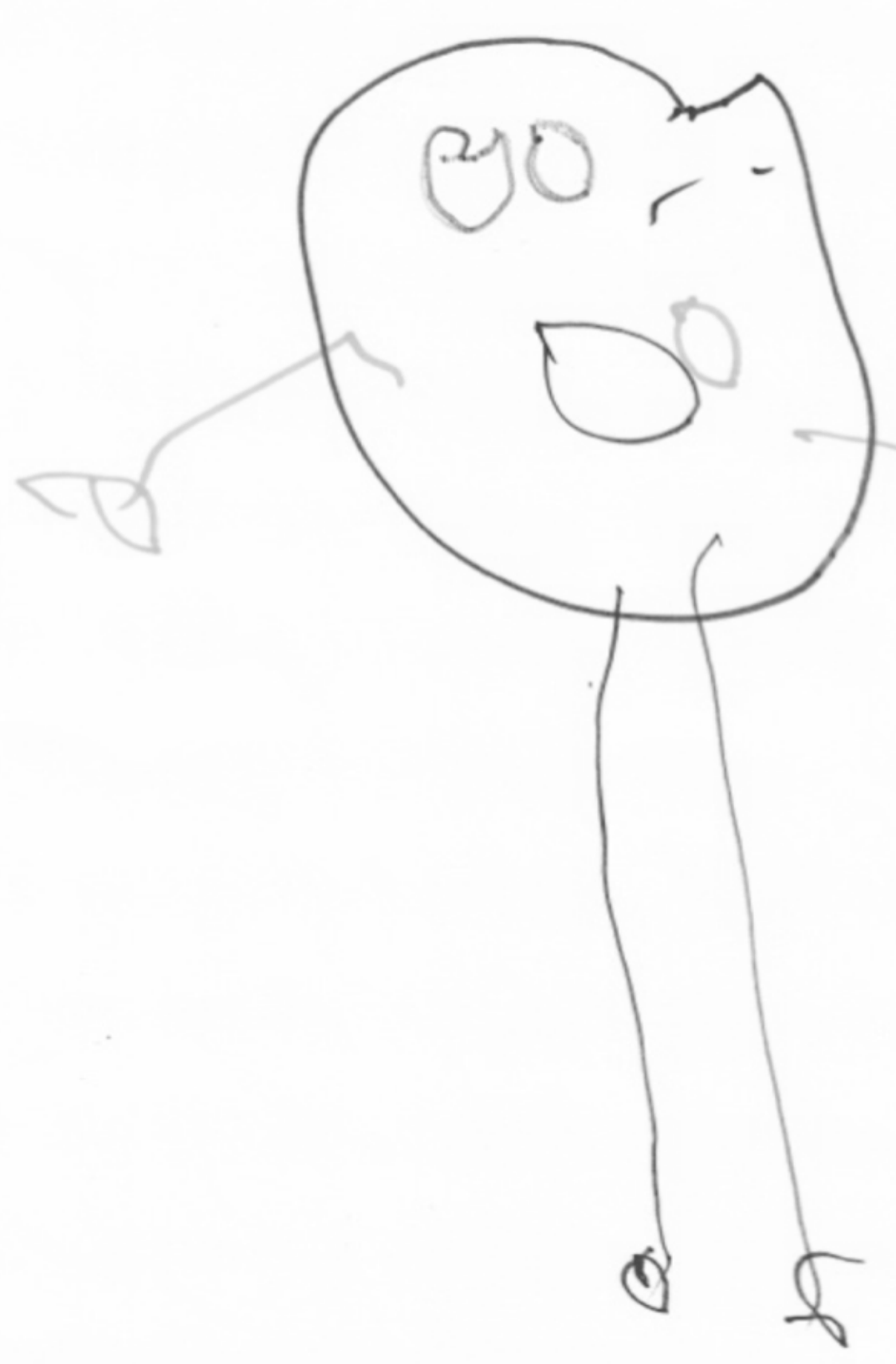




\section{Chapter 1}

General introduction 

Duchenne (DMD) and Becker muscular dystrophy (BMD) are recessive $x$-linked (Xp21) neuromuscular disorders caused by mutations in the dystrophin (DMD) gene that encodes the dystrophin protein. ${ }^{1,2}$ The $D M D$ gene consists of 79 exons and contains at least eight independent tissue-specific isoform promoters. ${ }^{3,4}$ The full length dystrophin protein (Dp427) for instance is located at the inner part of the muscle fibre membrane (striated muscle) and here it interacts with the dystrophin-associated glycoprotein complex (DCG), which is responsible for the stability of muscle fibres during contraction. ${ }^{5}$ In DMD, an absence of the functional full-length dystrophin protein in muscles, results in progressive and severe muscle weakness, respiratory and cardiac complications leading to a life expectancy limited to early adulthood ${ }^{6,7}$ In the milder variant, BMD, the full-length dystrophin protein is partially functional resulting in a heterogenous disease course with features such as, incidental muscle cramps and great life expectancy to severe muscle weakness at adolescents with or without cardiomyopathy. ${ }^{1}$

Expression of the full length dystrophin is also found in the brain (Dp427B) were it anchors a subset of $\mathrm{GABA}_{A}$ receptors at the post-synaptic membrane of neurons. ${ }^{8}$ It is located in the hippocampus, amygdala and cerebral cortex with a slightly higher expression in the temporal and prefrontal cortex than in parietal and occipital cortex. $^{8-11}$ In addition to the full length dystrophin protein (Dp427), many different (shorter) isoforms exist and these are located in the retina (Dp260), in Schwann cells of peripheral nerves (Dp116), in the brain and kidney (Dp140) and an ubiquitously expression (incl. brain) is described for Dp71/Dp40 (see Figure 1.1 for an overview). ${ }^{8,12}$

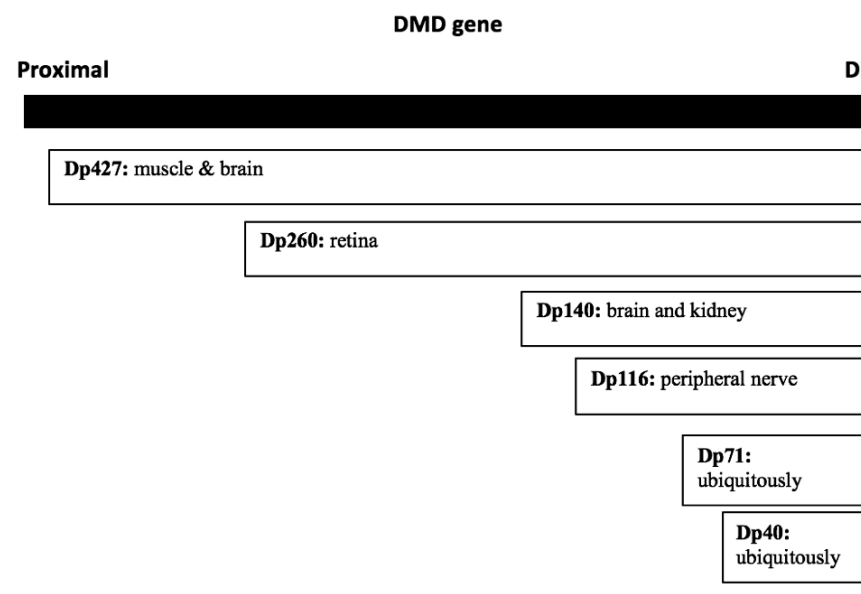

Figure 1.1 Schematic overview of the DMD gene, the dystrophin isoforms and their expression. The image was based on the references: Doorenweerd (2017), Muntoni (2003), and Waite (2012). ${ }^{8,12,18}$ 
The brain isoform Dp140 is linked to microvascular glial cells and is suggested to play a role in transcription factor activity, dendritic development, neuron differentiation and chromatin modification., ${ }^{8,13}$ Dp71/Dp40 are found in neurons and glial cells, more specifically Dp71 is found at specialized end-feet of perivascular astrocytes were it anchors aquaporin 4 receptors and Dp40 is localized at presynaptic and post-synaptic membranes in central excitatory synapses. ${ }^{8,14,15}$ The expression of the brain isoforms show a clear distinction. Dp427 has a relatively low expression throughout life, Dp140 is highly expressed during fatal life stages and Dp71 has an intermediate expression at all life stages. ${ }^{11,16}$ In contrast to the muscle DGC complex, different brain DCG complexes exists due to the various brain isoforms (Dp427, Dp140, Dp71/40). ${ }^{8,17}$ These brain DCG complexes play a crucial role in brain development and functioning due their roles in neuron differentiation and axon guidance. ${ }^{11}$

\section{Neurocognition and behaviour}

In addition to the skeletal muscle pathology, brain-related comorbidities such as neurocognitive and behavioural problems, learning disabilities and epilepsy may be an important part of the disease spectrum of both DMD and BMD. In Duchenne, $30 \%$ of patients have a full-scale intelligence quotient (FSIQ) score of approximately one standard deviation below the normal population (IQ <70) with more difficulties in verbal intelligence (VIQ) than performance intelligence (PIQ). ${ }^{19,20}$ Moreover, frequent reported cognitive deficiencies are limited verbal span capacity/verbal (working) memory deficiencies and problems with language (receptive and expressive), attention, executive functioning, visuo-spatial abilities and fine-motor skills. ${ }^{21-26}$ Longitudinal information on cognitive development is lacking, but the cognitive problems in DMD seem to be non-progressive. ${ }^{27-31}$ Furthermore, high prevalence rates of neurodevelopmental and behavioural disorders are described for DMD compared to the general population, including attention-deficit hyperactivity disorder (ADHD: 11.7-32\%) ${ }^{32-34}$, autism spectrum disorders (ASD: $\left.3.1-21 \%\right)^{33-36}$, obsessive-compulsive disorder (OCD: $4.8-5.1 \%)^{33,34}$ and anxiety disorders (27\%). ${ }^{34}$ High rates of psychosocial problems are also found in DMD (30-50\%), although these rates are comparable to those described for other chronic diseases. ${ }^{37-40}$ In addition, learning disabilities (LD) including verbal difficulties such as reading disabilities and dyslexia, as well as nonverbal learning disabilities such as math disabilities and dyscalculia frequently occur in DMD. $^{41-44}$ For instance, up to $40 \%$ of patients with DMD have reading disabilities. ${ }^{26,41,43,45,46}$ 
In $\mathrm{BMD}$, only five studies focused on cognition, behaviour and learning. In contrast to DMD, it seems that BMD patients have FSIQ scores within the normal range (means reported IQs range from 87.8 to 95.6$).{ }^{47-49}$ With respect to cognitive features, previous studies reported more problems in attention, language/speech delays (35.7\%), and academics skills such as reading $(21 \%)$, spelling $(32 \%)$ and math $(26 \%)$ in BMD compared to the general population. ${ }^{48,50}$ Furthermore, higher prevalence rates of behavioural problems including hyperactive/inattention features (11-36\%), autistic features (11.4-17.6\%), aggressive behaviour, OCD (1.5-20\%), anxiety (21.4-50\%) and depression (2.2-8\%) have been noticed in BMD. ${ }^{47,48,50,51}$ Nevertheless, the reported findings of previous studies were predominantly based on young patients, retrospective cases or unstandardized questionnaires instead of on neurocognitive and behavioural evaluations.

The severity of neurocognitive impairments, behavioural disorders, and learning disabilities in DMD and BMD are likely related to the location of mutation and it effect on the expression of specific brain dystrophin isoforms. ${ }^{11,32,35,42,49,52-54}$ In Duchenne, it seems that patients with mutations affecting multiple brain isoforms (i.e. the full-length Dp427B and shorter brain isoforms including Dp140 and/or Dp71) display more severe brain-related comorbidities than patients missing only Dp427B. ${ }^{11,32,35,42,52-54}$ In Becker, the role of partial functional brain dystrophin expression on neurocognitive and behavioural functioning is limited. Only the study by Bardoni and colleagues found that a lack of the brain isoform, Dp140, in addition to Dp427B was related to lower general intellectual abilities of patients with $\mathrm{BMD} .^{49}$ No relation is found between other neurocognitive disfunctions or behavioural disorders and an aberrant expression of the brain isoforms in BMD patients. For both DMD and BMD additional information on the existence of specific dystrophin-associated neurocognitive impairments and behavioural disorders is recommended.

\section{Diagnostic work-up}

Improved medical care (e.g. improved ventilation assistance) has increased the life expectancy in DMD, but patients are also confronted with long-lasting or new mental health issues that negatively influence their quality of life. ${ }^{55}$ Thereby, improvements in screening and diagnostics of brain-related comorbidities are crucial. Across the lifespan, clinicians should be aware of possible neurocognitive impairments, neurodevelopmental and behavioural disorders, psychosocial problems and learning disabilities. ${ }^{55}$ The DMD standards of care recommended that at each visit and 
particularly during transition phases, the mental health status of patients should be screened. ${ }^{55}$ Further assessment should by applied when screening results display mental health problems. ${ }^{55}$ However, until now various methods have been used to evaluate behavioural and psychosocial problems in both DMD and BMD, leading to a variability in prevalence rates. An overview of adequate instruments and their psychometric quality for both populations is lacking.

\section{Interventions}

Further research on interventions that may limit the influence of brain-related comorbidities in neurocognition and behaviour is necessary and may increase the quality of life of DMD and BMD patients. In the DMD standards of care, recommendations were given on applying known evidence-based interventions to treat behavioural disorders which have been used in populations with other chronic (medical) conditions. ${ }^{55}$ They for instance considered psychopharmacological medication to treat moderate to severe symptoms of neurodevelopmental or behavioural disorders. ${ }^{55}$ Psychopharmacological medication should be applied according to the prescribing guidelines, although special attention must be given to the DMD patients' medical condition i.e. cardiac status, medical interactions and side effects when it is combined with other existing medication, particularly when patients are older. ${ }^{55}$ Stimulant medication is for instance one of the recommended psychopharmacological treatments for DMD patients who have a comorbid ADHD diagnosis. ${ }^{55}$ However the safety and effectiveness of this medication has not been evaluated scientifically previously. Furthermore, the DMD standards of care recommended that neuropsychological and educational interventions should be developed and implemented at home and school. However, current literature lacks information on the effectiveness of these interventions for DMD. 


\section{Aims and outlines of this thesis}

The main aim of this thesis was to provide insights on neurocognition and behaviour, diagnostic work-up and interventions in Duchenne and Becker muscular dystrophy. To achieve this, this thesis addresses the following research questions:

1. Is there a DMD specific dystrophin-associated neurocognitive and behavioural profile?

2. Are cognitive deficits in DMD stable over time?

3. What are the neurocognitive and behavioural features of paediatric and adult males with BMD and are these related to disease severity?

4. Which instruments are valid and reliable to assess behavioural and psychosocial functioning in DMD and BMD?

5. Do males with DMD benefit from psychological (cognitive) interventions such as computerized working memory training?

6. Is stimulant medication i.e. short-acting methylphenidate a safe and effective psychopharmacological treatment for males with DMD and a comorbid ADHD diagnosis? 


\section{Outline}

\section{Neurocognition and behaviour}

- In chapter 2, we evaluate the neurocognitive and behavioural profiles of two neurogenetic disorders, DMD and Neurofibromatosis type 1.

- In chapter 3, we longitudinally assess cognitive functions and explore the role of Dp140 in DMD neurocognitive performance.

- In chapter 4, we describe the neurocognitive and behavioural features of adult males with BMD and correlate these to disease severity.

- In chapter 5, we evaluate the neurocognitive and behavioural profiles of three brothers with BMD with a similar dystrophin gene mutation.

\section{Diagnostic work-up of behavioural and psychosocial assessment}

- In chapter 6, we systematically review studies investigating behavioural and psychosocial functioning in DMD and BMD. Our main aims were to give an overview of the instruments used and their psychometric quality.

\section{Interventions}

- In chapter 7, we explore the benefits of computerized working memory training in males with DMD and a comorbid learning disability.

- In chapter 8, we evaluate the safety and effectiveness of short acting methylphenidate for males with DMD and a comorbid ADHD diagnosis. 


\section{References}

1. Bushby K, Gardner-Medwin D, Nicholson L, et al. The clinical, genetic and dystrophin characteristics of Becker muscular dystrophy. J Neurol 1993;240:105-112.

2. Hoffman EP, Brown Jr RH, Kunkel LM. Dystrophin: the protein product of the Duchenne muscular dystrophy locus. Cell 1987;51:919-928.

3. Roberts RG, Coffey AJ, Bobrow M, Bentley DR. Exon structure of the human dystrophin gene. Genomics 1993;16:536-538.

4. Blake DJ, Weir A, Newey SE, Davies KE. Function and genetics of dystrophin and dystrophin-related proteins in muscle. Physiol Rev 2002;82:291-329.

5. Ehmsen J, Poon E, Davies K. The dystrophin-associated protein complex. J Cell Sci 2002;115:2801-2803.

6. Emery AE. The muscular dystrophies. Lancet 2002;359:687-695.

7. Mercuri E, Muntoni F. Muscular dystrophies. Lancet 2013;381:845-860.

8. Waite A, Brown SC, Blake DJ. The dystrophin-glycoprotein complex in brain development and disease. Trends Neurosci 2012;35:487-496.

9. Chamberlain JS, Pearlman JA, Muzny DM, et al. Expression of the murine Duchenne muscular dystrophy gene in muscle and brain. Science 1988;239:1416-1418.

10. Lidov $\mathrm{H}$, Byers $\mathrm{T}$, Kunkel L. The distribution of dystrophin in the murine central nervous system: an immunocytochemical study. Neuroscience 1993;54:167-187.

11. Doorenweerd N, Mahfouz A, van Putten M, et al. Timing and localization of human dystrophin isoform expression provide insights into the cognitive phenotype of Duchenne muscular dystrophy. Sci Rep 2017;7:12575.

12. Muntoni F, Torelli S, Ferlini A. Dystrophin and mutations: one gene, several proteins, multiple phenotypes. Lancet Neurol 2003;2:731-740.

13. Doorenweerd N. Combining genetics, neuropsychology and neuroimaging to improve understanding of brain involvement in Duchenne muscular dystrophy-a narrative review. Neuromuscul Disord 2020; 30(6):437-442.

14. Fujimoto T, Itoh K, Yaoi T, Fushiki S. Somatodendritic and excitatory postsynaptic distribution of neurontype dystrophin isoform, Dp40, in hippocampal neurons. Biochem Biophys Res Commun 2014;452: 79-84.

15. Lidov HG, Selig S, Kunkel LM. Dp140: a novel 140 kDa CNS transcript from the dystrophin locus. Hum Mol Genet 1995;4:329-335.

16. Morris G, Simmons C, Man N. Apo-Dystrophins (dp140 and dp71) and dystrophin-splicing isoforms in developing brain. Biochem Biophys Res Commun 1995;215:361-367.

17. Waite $A$, Tinsley $C L$, Locke $M$, Blake DJ. The neurobiology of the dystrophin-associated glycoprotein complex. Ann Med 2009;41:344-359.

18. Doorenweerd N. The Duchenne Brain. Leiden2017.

19. Cotton S, Voudouris NJ, Greenwood KM. Intelligence and Duchenne muscular dystrophy: Full-Scale, Verbal, and Performance intelligence quotients. Dev Med Child Neurol 2001;43:497-501.

20. Cotton SM, Voudouris NJ, Greenwood KM. Association between intellectual functioning and age in children and young adults with Duchenne muscular dystrophy: further results from a meta-analysis. Dev Med Child Neurol 2005;47:257-265.

21. Billard C, Gillet P, Signoret J, et al. Cognitive functions in Duchenne muscular dystrophy: a reappraisal and comparison with spinal muscular atrophy. J Neuromuscul Dis 1992;2:371-378.

22. Wicksell RK, Kihlgren M, Melin L, Eeg-Olofsson O. Specific cognitive deficits are common in children with Duchenne muscular dystrophy. Dev Med Child Neurol 2004;46:154-159.

23. Cyrulnik SE, Fee RJ, Batchelder A, Kiefel J, Goldstein E, Hinton VJ. Cognitive and adaptive deficits in young children with Duchenne muscular dystrophy (DMD). J Int Neuropsychol Soc 2008;14:853-861.

24. Hinton V, Fee R, Goldstein E, De Vivo D. Verbal and memory skills in males with Duchenne muscular dystrophy. Dev Med Child Neurol 2007;49:123-128.

25. Hellebrekers DM, Doorenweerd N, Sweere DJ, et al. Longitudinal follow-up of verbal span and processing speed in Duchenne muscular dystrophy. Eur J Paediatr Neurol 2020;25:120-126. 
26. Mento G, Tarantino V, Bisiacchi PS. The neuropsychological profile of infantile Duchenne muscular dystrophy. Clin Neuropsychol 2011;25:1359-1377.

27. Leibowitz D, Dubowitz V. Intellect and behaviour in Duchenne muscular dystrophy. Dev Med Child Neurol 1981;23:577-590.

28. Dorman C, Hurley AD, Avignon JD. Language and learning disorders of older boys with Duchenne muscular dystrophy. Dev Med Child Neurol 1988;30:316-327.

29. Snow WM, Anderson JE, Jakobson LS. Neuropsychological and neurobehavioral functioning in Duchenne muscular dystrophy: a review. Neurosci Biobehav Rev 2013;37:743-752.

30. Miller G, Tunnecliffe M, Psych MA, Douglas PS, Dip G. IQ, prognosis and Duchenne muscular dystrophy. Brain Dev 1985;7:7-9.

31. Prosser EJ, Murphy E, Thompson MW. Intelligence and the gene for Duchenne muscular dystrophy. Arch Dis Child 1969;44:221.

32. Pane $M$, Lombardo $M E$, Alfieri $P$, et al. Attention deficit hyperactivity disorder and cognitive function in Duchenne muscular dystrophy: phenotype-genotype correlation. J Pediatr 2012;161:705-709. e701.

33. Hendriksen JG, Vles JS. Neuropsychiatric disorders in males with Duchenne muscular systrophy: frequency rate of attention-deficit hyperactivity disorders (ADHD), autism spectrum disorder, and obsessive-compulsive disorder. J Child Neurol 2008;23(5):477-481.

34. Banihani R, Smile S, Yoon G, et al. Cognitive and neurobehavioral profile in boys with Duchenne muscular dystrophy. J Child Neurol 2015;30:1472-1482.

35. Ricotti V, Mandy WP, Scoto M, et al. Neurodevelopmental, emotional, and behavioural problems in Duchenne muscular dystrophy in relation to underlying dystrophin gene mutations. Dev Med Child Neurol 2016;58:77-84.

36. Colombo P, Nobile M, Tesei A, et al. Assessing mental health in boys with Duchenne muscular dystrophy: Emotional, behavioural and neurodevelopmental profile in an Italian clinical sample. E Eur J Paediatr Neurol 2017;21(4):639-647.

37. Thompson Jr RJ, Zeman JL, Fanurik D, Sirotkin-Roses M. The role of parent stress and coping and family functioning in parent and child adjustment to Duchenne Muscular Dystrophy. J Clin Psychol 1992;48: 11-19.

38. Uzark K, King E, Cripe L, et al. Health-related quality of life in children and adolescents with Duchenne muscular dystrophy. Pediatrics 2012;130:e1559-e1566.

39. Hendriksen JG, Poysky JT, Schrans DG, Schouten EG, Aldenkamp AP, Vles JS. Psychosocial adjustment in males with Duchenne muscular dystrophy: psychometric properties and clinical utility of a parent-report questionnaire. J Pediatr Psychol 2009;34:69-78.

40. Darke J, Bushby K, Le Couteur A, McConachie H. Survey of behaviour problems in children with neuromuscular diseases. Eur J Paediatr Neurol 2006;10:129-134.

41. Hendriksen JG, Vles JS. Are males with Duchenne muscular dystrophy at risk for reading disabilities? Pediatr Neurol 2006;34:296-300.

42. Wingeier K, Giger E, Strozzi S, et al. Neuropsychological impairments and the impact of dystrophin mutations on general cognitive functioning of patients with Duchenne muscular dystrophy. J Clin Neurosci 2011;18:90-95.

43. Billard C, Gillet $P$, Barthez MA, Hommet $C$, Bertrand P. Reading ability and processing in Duchenne muscular dystrophy and spinal muscular atrophy. Dev Med Child Neurol 1998;40:12-20.

44. Leaffer EB, Fee RJ, Hinton VJ. Digit span performance in children with dystrophinopathy: a verbal span or working memory contribution? J Int Neuropsychol Soc 2016;22:777-784.

45. Lorusso ML, Civati F, Molteni M, Turconi AC, Bresolin N, D'Angelo MG. Specific profiles of neurocognitive and reading functions in a sample of 42 Italian boys with Duchenne Muscular Dystrophy. Child Neuropsychol 2013;19:350-369.

46. Hinton V, De Vivo D, Fee R, Goldstein E, Stern Y. Investigation of poor academic achievement in children with Duchenne muscular dystrophy. Learn Disabil Res Pract 2004;19:146-154.

47. Mori-Yoshimura M, Mizuno Y, Yoshida S, et al. Psychiatric and neurodevelopmental aspects of Becker muscular dystrophy. Neuromuscul Disord 2019;29(12):930-939.

48. Young HK, Barton BA, Waisbren $\mathrm{S}$, et al. Cognitive and psychological profile of males with becker muscular dystrophy. J Child Neurol 2008;23:155-162. 
49. Bardoni A, Felisari G, Sironi M, et al. Loss of Dp140 regulatory sequences is associated with cognitive impairment in dystrophinopathies. Neuromusc Disord 2000;10:194-199.

50. Lambert JT, Darmahkasih AJ, Horn PS, et al. Neurodevelopmental, Behavioral, and Emotional Symptoms in Becker Muscular Dystrophy. Muscle Nerve 2020;61(2):156-162.

51. Mori-Yoshimura M, Mizuno $\mathrm{Y}$, Yoshida S, et al. Social involvement issues in patients with Becker muscular dystrophy: A questionnaire survey of subjects from a patient registry. Brain Dev 2018;40: 268-277.

52. Chamova T, Guergueltcheva V, Raycheva M, et al. Association between loss of dp140 and cognitive impairment in duchenne and becker dystrophies. Balkan J Med Genet 2013;16:21-29.

53. Thangarajh M, Hendriksen J, McDermott M, Martens W, Hart K, Griggs R. Relationships between DMD mutations and neurodevelopment in dystrophinopathy (P5. 4-002). Neurology 2019;93(17): e1597-e1604.

54. Doorenweerd N, Straathof CS, Dumas EM, et al. Reduced cerebral gray matter and altered white matter in boys with Duchenne muscular dystrophy. Ann Neurol 2014;76:403-411.

55. Birnkrant DJ, Bushby K, Bann CM, et al. Diagnosis and management of Duchenne muscular dystrophy, part 3: primary care, emergency management, psychosocial care, and transitions of care across the lifespan. Lancet Neurol 2018;17(5):445-455. 


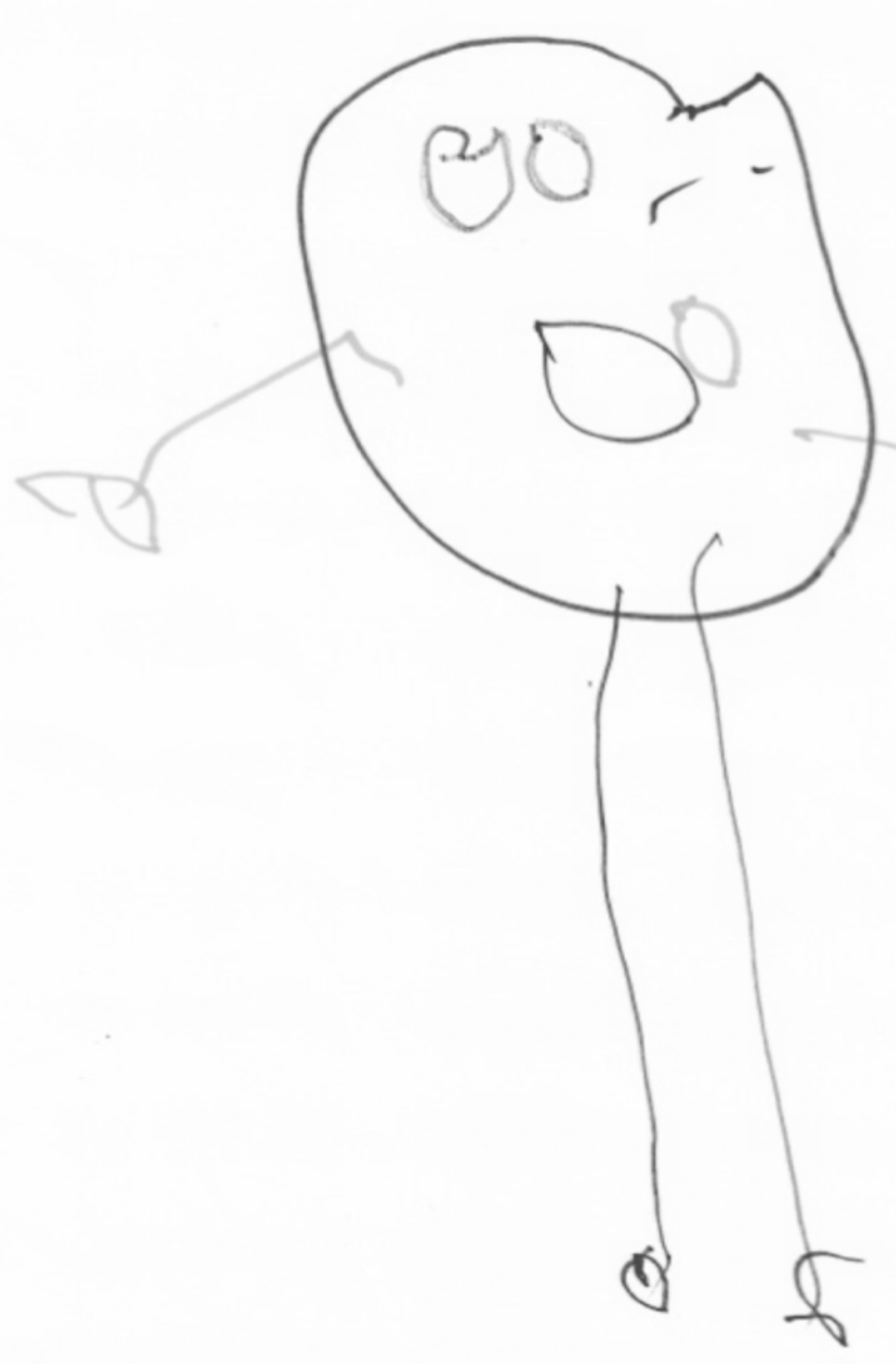




\section{Chapter 2}

Cognitive and behavioural functioning in two neurogenetic disorders; how different are these aspects in Duchenne muscular dystrophy and Neurofibromatosis type 1 ?

Danique M.J. Hellebrekers, Sandra A.M. van Abeelen, Coriene Catsman, Sander M.J. van Kuijk, Annick M. Laridon, Sylvia Klinkenberg, Jos G.M. Hendriksen, Johan S.H. Vles Submitted Journal of the International Neuropsychological Society 


\section{Abstract}

The presence of neurocognitive and behavioural problems are common features in various neurogenetic disorders. In Duchenne muscular dystrophy (DMD), these problems have been linked to mutations along the dystrophin gene affecting different brain dystrophin isoforms. However, comparable cognitive and behavioural problems have been found in Neurofibromatosis type 1 (NF1). This study aims to assess disorder specific differences in cognition and behaviour between DMD and NF1. At total of 38 male patients with DMD were aged-matched to 38 male patients with NF1. Patients of both groups underwent neurocognitive assessment for regular clinical care. Intellectual abilities, sequential and simultaneous processing, verbal memory and sustained attention were evaluated. In addition, parents and teachers completed behavioural questionnaires. Males with DMD exhibited low intellectual abilities and sequential processing problems, but these outcomes not significantly differed from males with NF1. Simultaneous processing, verbal memory and sustained attention outcomes were equal for both groups. Outcomes of questionnaires displayed higher rates of aggressive behaviour (13.2\%) in DMD, whereas in NF1 higher rates of problems with thinking (15.8\%), withdrawn (10.5\%) and social behaviour (10.5\%) were noticed. In the neurogenetic disorders DMD and NF1, on average overlapping cognitive and behavioural problems are noticed, suggesting that these are not only caused by gene mutations resulting in a lack of one specific protein. Furthermore, due to the variability in severity and overlap of problems in both populations, (re) evaluation and monitoring of cognition and behaviour are important aspects for clinical care. 


\section{Introduction}

There is growing evidence that gene mutations can cause abnormal brain development that lead to cognitive and behavioural problems in patients with neurogenetic disorders such as Duchenne muscular dystrophy (DMD), Neurofibromatosis type 1, 22q11.2 deletion syndrome, Prader-Willi syndrome, fragile $X$ syndrome and Turner syndrome. ${ }^{1-6}$ In $\mathrm{DMD}$, gene mutations result in a loss of the full-length dystrophin protein isoform (Dp427) in muscles (M) and the brain (B). ${ }^{7,8} A$ lack of the dystrophin protein Dp427 $7_{M}$ is responsible for the progressive muscle weakness in $D M D{ }^{9}$ The isoform Dp427 $\mathrm{B}$ and three shorter brain isoforms Dp140, Dp71+Dp40 are believed to be expressed throughout the cerebral cortex with the highest expression in the temporal and frontal cortex, the amygdala and hippocampus. ${ }^{10-13}$ The production of one of the brain isoforms (Dp140) is particularly elevated during foetal life stages, suggesting that it may influence brain development. ${ }^{11}$

Patients with Duchenne frequently exhibit cognitive problems, neurodevelopmental-, and behavioural disorders. ${ }^{3,10,14,15}$ The full-scale intelligence quotient (FSIQ) in DMD is on average one standard deviation below the population mean. ${ }^{16}$ In addition, problems with verbal working memory, attention, executive functioning, learning (e.g. reading, writing, math) have been reported. ${ }^{14,16-18}$ The higher rates of neurodevelopmental and behavioural disorders are found for attention-deficit hyperactivity disorders (ADHD; up to $32 \%$ ), autism spectrum disorders (ASD; up to $21 \%$ ), obsessive compulsive disorders (OCD up to $5.1 \%$ ) and anxiety (up to $27 \%$ ). ${ }^{14,19-22}$ The numbers vary due to use of various (screening) instruments. Recent studies have tried to assess whether specific gene mutations affecting the production of brain isoforms can be related to the cognitive problems, neurodevelopmental-, and behavioural disorders of patients with DMD. ${ }^{10,11,14,21-24}$ It seems that patients with mutations affecting multiple brain isoforms exhibit more severe problems in cognition and behaviour than patients missing only Dp427 ${ }^{19,21,23-26}$

Neurofibromatosis type 1 (NF1) is caused by germline mutations in the NF1 gene, resulting in a decreased production of the tumour suppressive protein, neurofibromin. ${ }^{27}$ There are a broad number of possible mutations in the large NF1 gene, resulting in variable phenotypes with various neurocutaneous manifestations including (plexiform) neurofibromas, café-au-lait spots, skinfold freckling, but also skeletal and muscular problems (e.g. scoliosis, pseudo-arthrosis, decreased bone strength, reduced muscle strength and motor problems). ${ }^{27,28}$ Previous studies in mice have showed that deletions involving exons NF1-23a and NF1-9a result in altered 
isoform expression in the brain (i.e. astrocytes) and in the central nervous systems (i.e. in neurons of striatum, cortex and hippocampus). ${ }^{29}$ Due to the role of neurofibromin in the brain, human and mice studies have linked a lack of this protein to the cognitive and learning disabilities that are found for NF1. ${ }^{29-32}$ Low-average IQ levels are usually shown in NF1 patients, but impairments are also found in visuo-spatial perceptual and visuomotor skills, language, learning (e.g. reading) and executive functions (e.g. attention and working memory). ${ }^{31-35}$ In addition, in NF1 higher prevalence rates of ADHD (up to $50 \%$ ), ASD (14\%) and behavioural problems such as anxiety and depression (43\%), have been noticed compared to the general population. ${ }^{31-33,35-40}$ Due to the large number of unique mutations in NF1, it is complicated to define a distinct cognitive and behavioural profile. ${ }^{31}$ However, recent studies have revealed that patients with microdeletions display more pronounced cognitive impairments and learning disabilities than patients with intragenic mutations. ${ }^{31,41-43}$

For neuropsychological diagnostic and treatment purposes we were interested whether patients with different neurogenetic disorders such as DMD and NF1 have specific cognitive and behavioural profiles. Therefore, the current study aimed to assess whether the cognitive and behavioural impairments differ between DMD and NF1.

\section{Materials and methods}

\section{Study population}

Eligible patients for current study were males with DMD and males with NF1 attending to the outpatient clinic of Kempenhaeghe, the Centre for Neurological Learning Disabilities (CNL), Heeze, the Netherlands, as this Centre is predominantly responsible for the neuropsychological care of these patients in the Netherlands. The inclusion criteria comprised of having a previously genetical confirmed mutation of the dystrophin gene for DMD patients or a previously genetical confirmed mutation of the neurofibromin gene for NF1 patients, an age between 6-16 years, an adequate proficiency in Dutch, normal hearing, absence of severe visual impairment and no physical immobility of upper extremities (the reliability of the cognitive tests may be reduced by impairments in hearing, vision and physical immobility of upper extremities). Exclusion criteria were: epilepsy, symptomatic optic pathway glioma, brain tumours, or hydrocephalus. NF1 males with focal abnormal signal intensity (FASI) were not excluded because no equivocal relation is found between the presence of FASI and cognitive, developmental impairments and learning disabilities. ${ }^{44,45}$ Each eligible male 
patient with DMD was matched on age (restriction within 1 year) to an age equivalent male with NF1. The age range of participants (6-16 years) was chosen to allow for the administration of the cognitive test and behavioural questionnaires, standardized for the Dutch population. Ethical approval was granted by the local Medical Ethical Committee of Kempenhaeghe. The study was conducted in accordance with the $18^{\text {th }}$ World Medical Assembly, Helsinki 194.

\section{Study procedure}

DMD and NF1 patients received an extensive neuropsychological assessment between October 2008 and August 2019 to evaluate their cognitive and behavioural functioning as part of regular clinical care at CNL. Cognitive assessment evaluated intellectual abilities (full scale intelligence, verbal intelligence and performance intelligence), processing speed, sequential processing (verbal span capacity and working memory), simultaneous processing (visuospatial functioning), verbal memory (immediate recall, delayed recall, recognition) and sustained visual- and auditory attention. Behavioural functioning was screened using questionnaires for parents and teachers. All collected cognitive and behavioural data were extracted from the patient files for current retrospective study. Demographic (i.e. age, educational level, gender), disease-related characteristics (i.e. genetic mutation, ambulation, comorbid learning disabilities, neurodevelopmental or behavioural DSM classified diagnoses, use of stimulant medication such as methylphenidate $(\mathrm{MPH})$, use of corticosteroids, somatic comorbidities, vision or hearing problems and immobility of upper extremities), sociodemographic characteristics of parents and information on problems during pregnancy and delivery were extracted from the patient files. The comorbid learning disabilities extracted from the patient files included dyslexia and dyscalculia. In addition, learning difficulties such as problems with reading, writing, math, automatization or spelling that did not fulfil the criteria for dyslexia and dyscalculia were extracted from the files. The neurodevelopmental and behavioural DSM-IV/DSM5 that were obtained from the patient files included ADHD, ASD, OCD, developmental coordination disorder (DCD), anxiety, depression and tic disorders. All cognitive, behavioural and learning comorbidities were previously diagnosed by a health or medical professional. The educational status of patients was categorized as regular or special education. Parents educational status was indicated using the Dutch Verhage categories $^{46}$ and was used to estimate the sociodemographic status of patients. The Verhage categories were combined into (1) low level (i.e. <6 years of primary education, finished primary education, $<2$ years low-level secondary education, finished low-level secondary education), (2) middle level (i.e. finished average-level secondary 
education) and (3) high level (i.e. finished high level secondary education, university degree). ${ }^{46}$

\section{Neuropsychological assessment}

\section{Cognition}

The Wechsler Intelligence Scale for Children Third edition (WISC-III) ${ }^{47}$ measured FullScale Intelligence Quotient (FSIQ), Verbal Intelligence (VIQ), Performance Intelligence (PIQ), Verbal Comprehension, Perceptual Organization and Processing Speed. Raw scores of the WISC-III were converted to age-related norm scores (mean=100, SD=15). ${ }^{47}$ The Kaufmann Assessment Battery for Children-II (KABC-II) was used to assess sequential processing (verbal span and auditory working memory) and simultaneous processing (visuospatial functioning). ${ }^{48}$ Sequential processing was based on the subtests Number recall and Word Order. Simultaneous processing was based on the subtests Rover and depending on age the subtests Triangles ( 6 years) or Block Counting (7-16 years). Raw scores of the subtests were converted to age-related scaled scores (mean $=100, S D=15) .{ }^{48}$ Verbal memory of immediate recall, delayed recall and recognition was tested using the Rey auditory learning task (15-word test). ${ }^{49}$ Scores of the 15-word test were computed to (1) a sum of correct responses given during the five consecutive trials (total immediate recall score), (2) total correct response during the delayed trial (delayed recall score) and (3) sum of correct recognition responses (recognition score). ${ }^{49}$ Sustained visual attention was measured using the Bourdon Vos. $^{50}$ The Test of Everyday Attention for Children, Second Edition (TEA-Ch) ${ }^{51}$, subtest Score! was used to measure sustained auditory attention. Teach-Ch raw scores were converted to scaled scores (mean $=10, S D=3$ ). ${ }^{51}$

\section{Behaviour}

Behavioural functioning was screened using two informant rating instruments, the Child Behaviour Checklist for Children (CBCL) and the Teacher report Form (TRF). ${ }^{52}$ Both instruments evaluated behaviour based on eight syndrome scales (anxious/depressed, withdrawn/depressed, somatic complaints, social problems, thought problems, attention problems, rule-breaking behaviour and aggressive behaviour). Two broadband scales on internalizing symptoms (made up of withdrawn, somatic complaints and anxious/depressed scales), externalizing symptoms (made up of rulebreaking behaviour and aggressive behaviour), and a total problem scale score were calculated using the syndrome scale scores. In line with the manual, a cut-off value (clinical range score) of $\mathrm{T} \geq 70$ was used to indicate the clinical range of the eight 
syndrome scales, and $\mathrm{T} \geq 64$ was applied to indicate the clinical range of internalizing, externalizing symptoms and a total problem score. ${ }^{52}$

\section{Statistical analysis}

Age-matching (restriction within 1 year) was randomly performed by case control matching of SPSS. Demographic and disease-related characteristics were presented as mean (SD), or absolute number and proportion. Stochastic regression imputation was applied in case of incomplete variables of cognitive and behavioural data. ${ }^{53}$ The imputed values were drawn using predictive mean matching. ${ }^{53}$ Differences between the DMD and NF1 group on demographic and disease-related parameters as well as cognitive and behavioural outcomes were tested using the independent samples t-test, $\mathrm{X}^{2}$ tests, Fisher exact test, or Mann-Whitney- $U$ tests, as appropriate. Effect sizes (quantified as Cohen's d) were calculated to indicate the strength of differences of the cognitive and behavioural outcomes. ${ }^{54}$ Effect sizes were defined as: $0.20-0.50=$ small, $0.50-0.80=$ medium and $\geq 0.80=$ large. ${ }^{54}$ Multivariate analyses (MANOVA) examined differences between the groups on cognitive and behavioural outcomes corrected for the covariates age, comorbid diagnoses of patients (i.e. learning, neurodevelopmental, or behavioural disorders), use of stimulant medication (MPH), use of steroids, educational status of patients and family history of learning and behavioural problems. Preliminary assumptions associated with all test statistics, such a normality and multivariate normality, homogeneity of variance, homogeneity of variance-covariance matrices, linearity and multicollinearity were examined using a variety of methods including visual inspection of histograms, boxplots, scatterplots, inspection of skewness, kurtosis, Shapiro-Wilk test, Levene's test and Box's M test $(p \geq .001){ }^{55}$ Cognitive outcomes i.e. age-related norm scores were converted to z-scores (mean $=0$, $S D=1)$. Behavioural outcomes were also evaluated using the clinical cut-off value of Tscore $\geq 63 .^{52}$ All statistical analyses were carried out using IBM SPSS version 24.0 for MAC OS $X$.

\section{Results}

\section{Participant characteristics}

Data of 50 patients with DMD were available. Twelve patients were excluded because of age ( $n=7$ were $<6$ years and $n=2$ were $>16$ years old) or having epilepsy $(n=3)$. In total 38 patients with DMD were age-matched with 38 males with NF1. Demographic and disease-related characteristics of both groups are displayed in Table 2.1. 
Table 2.1 Participant characteristics.

\begin{tabular}{|c|c|c|c|}
\hline & $\begin{array}{c}\text { DMD } \\
(\mathrm{N}=38)\end{array}$ & $\begin{array}{c}\text { NF1 } \\
(\mathrm{N}=38)\end{array}$ & $\mathbf{p}$ \\
\hline \multicolumn{4}{|l|}{ Demographic characteristics } \\
\hline Mean age in years $(S D)$ & $9.6(2.6)$ & $9.7(2.6)$ & .839 \\
\hline Education of participants (\%) & & & $.000^{* *}$ \\
\hline Regular education & $6(15.8)$ & $23(60.5)$ & \\
\hline Special education & $32(84.2)$ & $15(39.4)$ & \\
\hline \multicolumn{4}{|l|}{ Educational levels of parents (\%) } \\
\hline Mother: & & & .522 \\
\hline Low level & $6(17.1)$ & $4(10.5)$ & \\
\hline Middle level & $14(40.0)$ & $17(56.7)$ & \\
\hline High level & 15 (42.9) & $9(30.0)$ & \\
\hline Father: & & & .570 \\
\hline Low level & $4(10.5)$ & $2(7.1)$ & \\
\hline Middle level & $6(19.4)$ & $10(35.7)$ & \\
\hline High level & $21(67.7)$ & $16(57.1)$ & \\
\hline Family history learning and behavioural problems (\%) & & & $.002^{*}$ \\
\hline ADHD & $4(10.8)$ & $9(24.3)$ & \\
\hline ASS & $1(2.7)$ & $4(10.8)$ & \\
\hline Dyslexia & $6(16.2)$ & $13(35.1)$ & \\
\hline Learning difficulties & $3(8.1)$ & $3(8.1)$ & \\
\hline Pregnancy \& delivery problems (\%) & & & .229 \\
\hline Hypoxia & $1(2.6)$ & 0 & \\
\hline Premature birth ( 34 to $\leq 37 \mathrm{wk}$ ) & $4(10.4)$ & $1(2.6)$ & \\
\hline C-section & $1(2.5)$ & $2(5.3)$ & \\
\hline Intrauterine growth problems & $2(5.3)$ & $2(5.3)$ & \\
\hline Pre-eclampsia & $1(2.6)$ & 0 & \\
\hline \multicolumn{4}{|l|}{ Disease-related characteristics } \\
\hline Wheelchair dependence (\%) & & & $.000^{* *}$ \\
\hline Permanent & $16(44.4)$ & 0 & \\
\hline Intermittent & $5(13.9)$ & 0 & \\
\hline Never & $15(41.7)$ & 0 & \\
\hline \multicolumn{4}{|l|}{ Medication use (\%) } \\
\hline Steroids (prednisone) & $31(81.6)$ & 0 & $.000^{* *}$ \\
\hline Stimulants (MPH) & $4(10.5)$ & $9(23.7)$ & .222 \\
\hline Sleep problems (\%) & & & .133 \\
\hline Falling asleep & $8(21.6)$ & 15 (39.5) & \\
\hline Staying asleep & 0 & 0 & \\
\hline Comorbid diagnoses (\%) & & & .491 \\
\hline ADHD & $7(18.2)$ & $16(41.6)$ & $.025^{*}$ \\
\hline ADD & $3(7.9)$ & $4(10.5)$ & 1.000 \\
\hline ASD & $10(26.3)$ & $4(10.5)$ & .076 \\
\hline Depression & $1(2.6)$ & 0 & 1.000 \\
\hline Anxiety & $2(5.3)$ & 0 & .493 \\
\hline Tics & $1(2.6)$ & $1(2.6)$ & 1.000 \\
\hline Dyslexia & $1(2.6)$ & $4(10.5)$ & .358 \\
\hline Dyscalculia & $3(7.9)$ & 0 & .240 \\
\hline DCD & 0 & $11(28.9)$ & $.005^{*}$ \\
\hline
\end{tabular}

Results are mean (SD) or median (range) for continuous variables, and frequencies (\%) for categorical variables. Verhage categories are defined as low level (i.e. $<6$ years of primary education, finished primary education, $<2$ years low-level secondary education, finished low-level secondary education), middle level (i.e. finished average-level secondary education), and high level (i.e. finished high level secondary education, university degree). ${ }^{41}$ wk=weeks. Reasons for $\mathrm{C}$-section were: $\mathrm{N}=1$ pelvic presentation, $\mathrm{N}=1 \mathrm{C}$-section at 38 weeks because of intrauterine growth problems, and $\mathrm{N}=1$ emergency $\mathrm{C}$-section but reason was not documented. Reports on family history of learning and behavioural problems are based on $1^{\text {st }}, 2^{\text {nd }}$, and $3^{\text {rd }}$ family degree. * $p<.05$ (two-sided); ** $p<.01$ (two-sided). 
Of the DMD group, 21 males (55.3\%) had mutations affecting Dp140 production (i.e. mutations corrupting the Dp140 promoter, the Dp140 translation start site or were located downstream of exon 50 as the Dp140 ATG start-site is located in exon 51). Ten males (26.3\%) had mutations not affecting Dp140 production (i.e. deletions or duplications upstream of intron 44). Dystrophin expression was undefinable of five males (13.2\%) with deletions or duplication breakpoints between intron 44 and exon $51 .^{24}$ No information on deletions or duplications was available in the electronic patient files of two males (5.3\%). Of the NF1 group, none of the patients had microdeletions.

Disease-related characteristics are displayed in Table 2.1. The majority of the DMD group (81.6\%) used prednisone steroids, six (15.6\%) used deflazacort and one (2.6\%) had no corticosteroid treatment, because it severely affected his emotional status. The prevalence rates of comorbid diagnoses in neurodevelopmental and behavioural disorders differed between the DMD and NF1 group (see Table 2.1). ASD diagnoses were more often found for the DMD group (23.4\%) compared to the NF1 group (13\%), whereas the rate of ADHD diagnoses is higher for the NF1 group (41.6\%) than for the DMD group (18.2\%, see Table 2.1). Diagnoses of learning disorders such as dyslexia and dyscalculia were found in both groups. Furthermore, $n=11$ males with DMD (28.6\%) and $\mathrm{n}=18$ (46.8\%) males with NF1 exhibited learning disabilities in reading, writing, mathematics, spelling and automatization that not fulfil the diagnostic criteria for dyslexia or dyscalculia. Within the NF1 group nine used MPH, whereas in the DMD group four males used MPH (see Table 2.1).

\section{Cognitive outcomes}

\section{Intellectual abilities}

Of the DMD group, the mean FSIQ was 86.4 ( $S D=11.9$ ), mean Verbal IQ was 89.6 $(S D=12.0)$ and mean Performance IQ was 85.3 (SD=12.0). These two latest outcomes indicated no discrepancy between VIQ and PIQ for the DMD group. Additionally, their overall mean score of Verbal Comprehension was 91.5 ( $S D=9.5)$, mean Perceptual Reasoning was $84.9(S D=8.6)$ and mean Processing Speed was 89.5 (SD=16.2). Of the NF1 group, the overall mean FSIQ was 91.5 (SD=15.4), mean Verbal IQ was 93.9 $(S D=14.4)$ and mean Performance IQ was 89.9 ( $S D=17.0)$, signifying no discrepancy between VIQ and PIQ. The overall mean of the NF1 group for Verbal Comprehension was 95.5 ( $S D=15.0)$, mean Perceptual Reasoning was $89.4(S D=14.5)$ and mean Processing Speed was 94.0 (SD=17.3). As shown in Table 2.2, no significant differences were found on all IQ measures between the DMD and NF1 group. 
Table 2.2 Wechsler Intelligence Scale for Children-III outcomes of the DMD and the NF1 group.

\begin{tabular}{lccccccc}
\hline & $\begin{array}{c}\text { Mean (SD) } \\
\text { DMD group } \\
(\mathrm{N}=38)\end{array}$ & $\begin{array}{c}\text { Mean (SD) } \\
\text { NF1 group } \\
(\mathrm{N}=38)\end{array}$ & $\begin{array}{c}\text { Test-statistic } \\
\text { value }\end{array}$ & $\mathrm{p}$ & $\begin{array}{c}\text { Effect } \\
\text { size }\end{array}$ & & $95 \% \mathrm{Cl}$ \\
\hline & & & & & & Lower & Upper \\
\hline FSIQ & $-0.91(0.79)$ & $-0.57(1.03)$ & -1.626 & .108 & -0.4 & -0.76 & 0.07 \\
VIQ & $-0.69(0.81)$ & $-0.41(0.96)$ & -1.387 & .170 & -0.3 & -0.69 & 0.12 \\
PIQ & $-0.98(0.80)$ & $-0.67(1.13)$ & -1.370 & .175 & -0.3 & -0.76 & 0.14 \\
VC & $-0.57(0.63)$ & $-0.30(1.00)$ & -1.411 & .163 & -0.3 & -0.65 & 0.11 \\
PO & $-1.00(0.58)$ & $-0.71(0.96)$ & -1.654 & .103 & -0.4 & -0.72 & 0.00 \\
PS & $-0.77(1.12)$ & $-0.40(1.15)$ & -1.402 & .165 & -0.3 & -0.67 & 0.06 \\
\hline
\end{tabular}

Z-scores are mean (SD), Test statistic values are t-values, Effect size = Cohen's d, 95\% Cl=95\% Confidence Interval. $\mathrm{FSIQ}=$ Full-scale intelligence quotient, $\mathrm{VIQ}=$ Verbal intelligence quotient, $\mathrm{PIQ}=$ Performance intelligence quotient, $\mathrm{VC}=$ Verbal Comprehension, $\mathrm{PO}=$ Perceptual Organization, $\mathrm{PS}=$ Processing speed.

WISC distribution of FSIQ of the two groups are displayed in Figure 2.1. Of the DMD group, four males (10.5\%) had a FSIQ score of $\leq 70$, thirteen males $(34.2 \%)$ scored between $70-85$, seventeen $(44.7 \%)$ fell within the range $85-100$, three $(7.9 \%)$ scored between $100-115$ and one (2.6\%) had a FSIQ score $\geq 115$ (see Figure 2.1). Of the NF1 group, four $(10.5 \%)$ scored below $\leq 70$, six males $(15.8 \%)$ had a FSIQ score between 70-85, nineteen (50.0\%) fell within the range $85-100$, six (15.8\%) had a FSIQ score between $100-115$ and three (7.9\%) had FSIQ score $\geq 115$ (see Figure 2.1). The sociodemographic status (measured by educational status (ES) of parents) of both groups were not correlated to the FSIQ outcomes (ES mothers of DMD group $r s=.16$, $p>.05$, ES fathers of DMD group $r s=.19, p>.05$, ES mothers NF1 group, $r s=.13, p>.05$ and ES fathers NF1 group, $r s=.26, p>.05)$.

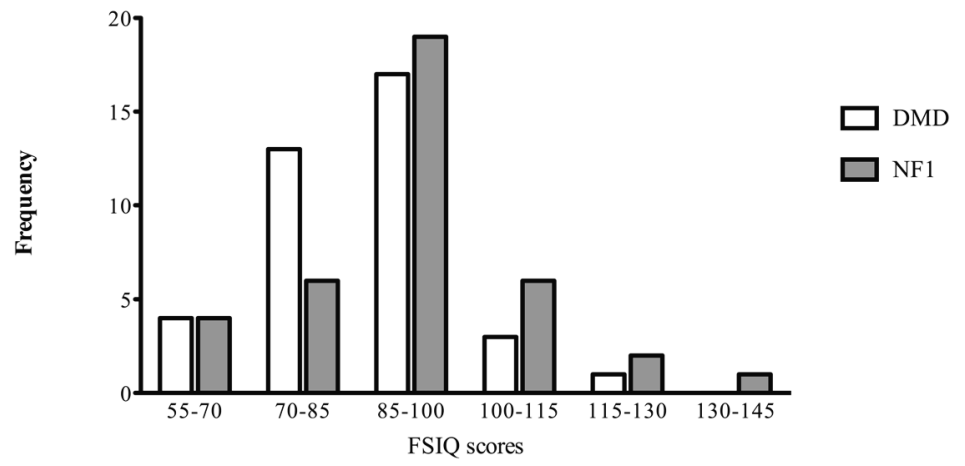

Figure 2.1 Frequencies of the Wechsler full-scale intelligence quotient scores of the DMD (N=38) and NF1 group (n=38). FSIQ=full-scale intelligence quotient, DMD=Duchenne muscular dystrophy, NF1= Neurofibromatosis type 1. 


\section{Sequential and simultaneous processing}

Results showed that mean sequential processing of the DMD group was 81.9 ( $S D=12.5$ ) and of the NF1 group mean sequential processing was 80.6 (SD=7.9). Mean simultaneous processing of the DMD group was 95.6 ( $S D=14.3)$ and of the NF1 group mean simultaneous processing was 97.5 ( $S D=12.0)$. No significant difference was found between the groups on sequential processing and simultaneous processing (see Table 2.3).

Table 2.3 Cognitive outcomes of (working) memory, attention, and visuospatial abilities of the DMD and NF1 group.

\begin{tabular}{lccccccc}
\hline $\begin{array}{l}\text { Cognitive } \\
\text { domains }\end{array}$ & $\begin{array}{c}\text { DMD } \\
(\mathrm{N}=38)\end{array}$ & $\mathrm{NF} 1$ & Test-statistic & $\mathrm{p}$ & Effect size & \multicolumn{2}{c}{$95 \% \mathrm{Cl}$} \\
\cline { 6 - 8 } SEQ & $-1.21(0.84)$ & $-1.29(0.53)$ & 0.536 & .594 & 0.1 & -0.23 & 0.41 \\
SIM & $-0.30(0.96)$ & $-0.17(0.80)$ & -0.620 & .537 & -0.1 & -0.53 & 0.28 \\
SVAS & $-0.55(1.01)$ & $-0.70(1.04)$ & 0.619 & .538 & 0.1 & -0.32 & 0.61 \\
SVAA & $-0.65(1.27)$ & $-0.51(1.36)$ & -0.468 & .641 & -0.1 & -0.74 & 0.46 \\
SAU & $-0.66(1.08)$ & $-0.85(1.05)$ & 0.767 & .445 & 0.2 & -0.30 & 0.67 \\
IR & $-0.27(1.17)$ & $-0.24(1.64)$ & -0.075 & .941 & -0.0 & -0.67 & 0.63 \\
DR & $-0.38(1.14)$ & $-0.54(1.35)$ & 0.542 & .589 & 0.1 & -0.42 & 0.73 \\
RC* & $28.5(24-30)$ & $29(21-30)$ & -0.890 & .374 & NA & NA & NA \\
\hline
\end{tabular}

Z-scores are mean (SD) except of * outcomes are raw median (range) scores, Test-statistic values are t-values, except of ${ }^{*}$ is z-value, Effect size=Cohen's d, 95\% Cl=95\% Confidence Interval. SEQ=Sequential processing, SIM=Simultaneous processing, SVAS=Sustained Visual Attention Speed, SVAA=Sustained Visual Attention Accuracy, SAU=Sustained Auditory Attention, IR=Immediate Recall, $D R=$ Delayed Recall, RC=Recognition, DMD=Duchenne muscular dystrophy, NF1=Neurofibromatosis type 1, NA=not applicable.

See Figure 2.2 for visualization of differences between the DMD and NF1 group on outcomes of sequential and simultaneous processing. Both groups had lower sequential processing than simultaneous processing outcomes (DMD group, $p<.001$ and NF1 group, $p<.001)$. No significant correlation was found between the lower sequential outcomes and FSIQ outcomes of the DMD population $(r=0.23, p>.05)$ and NF1 population $(r=0.05, p>$.05). Simultaneous processing outcomes were moderate but significantly correlated with FSIQ in the DMD group $(r=0.39, p<.05)$, but not in the NF1 group ( $r=0.08, p>.05)$. 


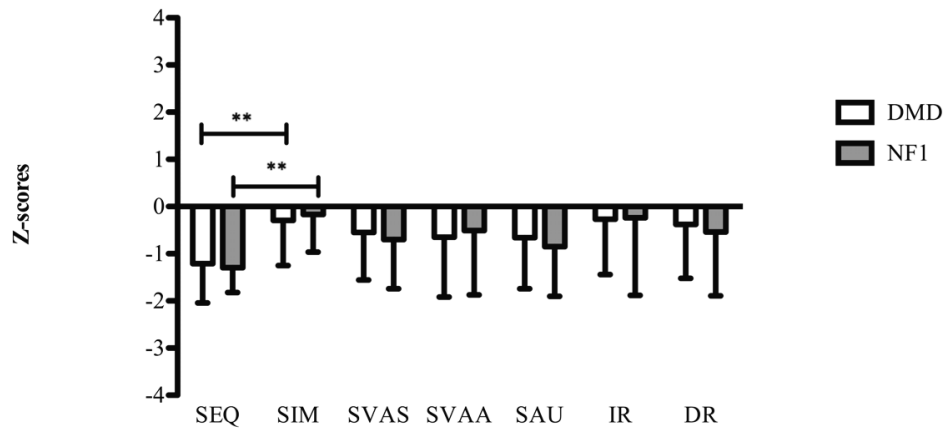

Figure 2.2 Mean (SD) outcomes of the DMD group $(N=38)$ and $N F 1(N=38)$ group. SEQ=Sequential processing, $\mathrm{SIM}=$ Simultaneous processing, SVAS=Sustained Visual Attention Speed, SVAA=Sustained Visual Attention Accuracy, SAU=Sustained Auditory Attention, IR=immediate recall, $D R=$ delayed recall, $D M D=D u c h e n n e$ muscular dystrophy, NF1=Neurofibromatosis type $1 .{ }^{* *} p<.001$ (two-sided).

\section{Verbal memory}

On verbal memory i.e. immediate recall, delayed recall and recognition no significant differences were found between the groups (see Table 2.3). The outcomes on immediate and delayed recall of both groups are visualized in Figure 2.2. Only immediate recall of the NF1 group was significantly correlated with total FSIQ $(r=0.55$, $p<.001)$ and no correlation was found for the DMD group $(r=0.06, p>.05)$.

\section{Sustained attention}

On visual sustained attention results showed no significant differences between the DMD and the NF1 group on speed or accuracy (see Table 2.3). Mean sustained auditory attention was also not significantly different between the groups (see Table 2.3). See Figure 2.2 for visualization of differences between the DMD and NF1 group on outcomes of sustained visual and auditory attention. All sustained attention measures were not significantly correlated with the FSIQ outcomes of the DMD and NF1 group.

A multivariate analysis was run to determine the effect of the covariates, age, comorbid diagnoses of patients (i.e. learning, neurodevelopmental, or behavioural disorders), use of stimulant medication (MPH), use of steroids, educational status of patients and family history of learning and behavioural problems on all cognitive outcomes of both groups. Results showed again non-significant differences between the groups on intellectual abilities, sequential and simultaneous processing, verbal memory, sustained visual and auditory attention after controlling for the influence of the covariates on the cognitive outcomes (see Table 2.4). 
Table 2.4 Multivariate analyses of cognitive outcomes of the DMD and NF1 group.

\begin{tabular}{|c|c|c|c|c|c|c|c|}
\hline \multirow[b]{3}{*}{ Cognitive outcomes } & \multicolumn{3}{|c|}{$\operatorname{DMD}(n=38)$} & \multicolumn{3}{|c|}{ NF1 $(n=38)$} & \multirow{3}{*}{$\mathrm{p}$} \\
\hline & \multirow[t]{2}{*}{ Mean } & \multicolumn{2}{|c|}{$95 \% \mathrm{Cl}$} & \multirow[t]{2}{*}{ Mean } & \multicolumn{2}{|c|}{$95 \% \mathrm{Cl}$} & \\
\hline & & Lower & Upper & & Lower & Upper & \\
\hline FSIQ & -0.52 & -1.01 & -0.03 & -0.93 & -1.40 & -0.45 & .359 \\
\hline VIQ & -0.22 & -0.68 & 0.25 & -0.87 & -1.32 & -0.41 & .121 \\
\hline PIQ & -0.72 & -1.26 & -0.19 & -0.91 & -1.43 & -0.39 & .698 \\
\hline VC & -0.27 & -0.70 & 0.16 & -0.55 & -0.97 & -0.13 & .470 \\
\hline $\mathrm{PO}$ & -0.88 & -1.33 & -0.43 & -0.80 & -1.20 & -0.36 & .841 \\
\hline PS & -0.60 & -1.22 & -0.03 & -0.52 & -1.13 & 0.09 & .883 \\
\hline SEQ & -1.22 & -1.60 & -0.84 & -1.28 & -1.65 & -0.91 & .854 \\
\hline SIM & -0.15 & -0.64 & 0.33 & -0.33 & -0.80 & 0.15 & .691 \\
\hline SVAS & -0.84 & -1.39 & -0.30 & -0.47 & -1.00 & 0.62 & .447 \\
\hline SVAA & -0.26 & -0.95 & 0.44 & -0.92 & -1.60 & -0.24 & .290 \\
\hline SAU & -1.18 & -1.74 & -0.61 & -0.25 & -0.81 & 0.30 & .073 \\
\hline IR & -0.06 & -0.81 & 0.30 & -0.47 & -1.21 & 0.27 & .548 \\
\hline DR & -0.16 & -0.85 & 0.53 & -0.64 & -1.32 & 0.03 & .433 \\
\hline
\end{tabular}

Cognitive outcomes are means of z-scores corrected for the covariates age, the presence of comorbidities (learning, neurodevelopmental or behavioural disorders), use of stimulant medication (MPH), use of steroids, educational status of patients, and family history of learning and behavioural problems. $95 \% \mathrm{Cl}=95 \%$ Confidence Interval. FSIQ=Full-Scale Intelligence quotient, VIQ=Verbal Intelligence quotient, $\mathrm{PIQ}=$ Performance intelligence quotient, $\mathrm{VC}=\mathrm{Verbal}$ Comprehension, $\mathrm{PO}=$ Perceptual Organization, $\mathrm{PS}=$ Processing speed, $\mathrm{SEQ}=$ Sequential processing, $\mathrm{SIM}=$ Simultaneous processing, SVAS=Sustained Visual Attention Speed, SVAA=Sustained Visual Attention Accuracy, SAU=Sustained Auditory Attention, $\mathrm{IR}=$ =immediate recall, $\mathrm{DR}=$ delayed recall, $\mathrm{DMD}=$ Duchenne muscular dystrophy, NF1=Neurofibromatosis type 1. $* p<.05$ (two-sided).

\section{Behavioural reports of parents and teachers}

Outcomes of the behavioural reports of the DMD and NF1 group are displayed in Table 2.5. Parents of the DMD group reported that $23.7-28.9 \%$ of the males had internalizing or externalizing problems, whereas according to teachers $13.2 \%$ displayed internalizing and externalizing problems. Aggressive behaviour was the most frequent observed behavioural problem in DMD (13.2\%) according to parents $(\mathrm{CBCL})$ responses. These five DMD males that displayed aggressive behaviour were aged between 7,1-14,4 years. Problems with thinking and withdrawn were also reported by parents of the DMD group. Results further showed differences in the prevalence rates of behavioural problems reported by parents (CBCL) compared to those reported by teachers (TRF), with limited behavioural problems documented by teachers. Parents of the NF1 group, reported that $18.4-15.8 \%$ of the males had internalizing and externalizing problems, which is approximately comparable to the responses of teachers $(15.8 \%$ internalizing and $15.8 \%$ externalizing). In particular, problems with thinking and withdrawn were documented by parents of the NF1 group, whereas teachers rated more social problems. 


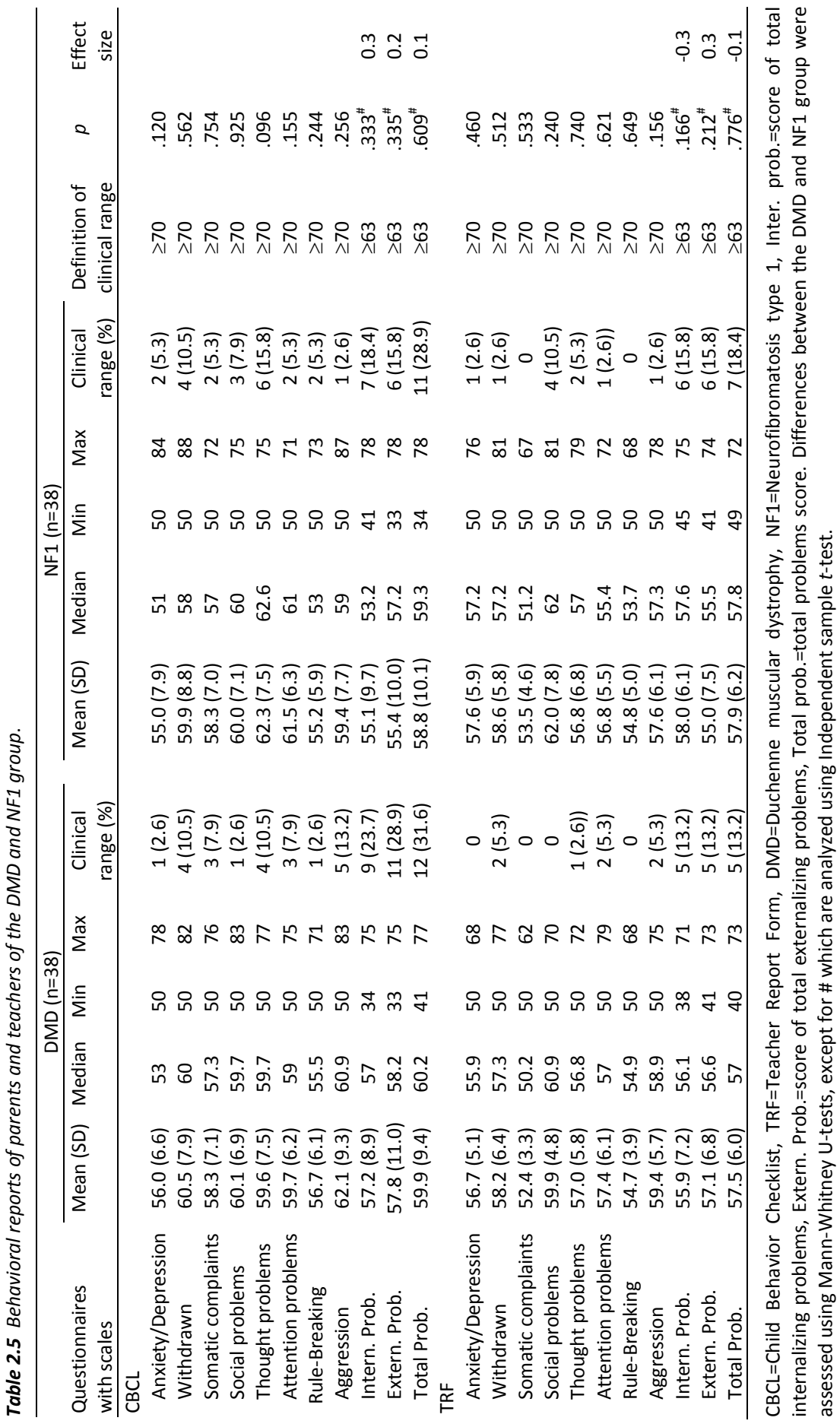


Again, a difference in rates was found between parents (CBCL) and teachers (TRF) responses for the NF1 group. No significant differences were found between the DMD and the NF1 group on all subscales, the broadband scales internalizing- and externalizing problems and total problem scores (see Table 2.5).

A multivariate analysis was run to determine the effect of the covariates, age, comorbid diagnoses of patients (i.e. learning, neurodevelopmental, or behavioural disorders), use of stimulant medication (MPH) or steroids, educational status of patients and family history of learning and behavioural problems on the $\mathrm{CBCL}$ and TRF broadband internalizing- and externalizing scales and the total problem scores. Results showed that the broadband internalizing scale of the $\mathrm{CBCL}$ was significantly higher for the DMD than for the NF1 group after controlling for the covariates (see Table 2.6). No differences were found between the groups on the internalizing TRF scale score, externalizing $\mathrm{CBCL}$ and TRF scale scores and the total problem scores of the $\mathrm{CBCL}$ and TRF (see Table 2.6).

Table 2.6 Multivariate analyses of behavioural reports of parents and teachers of the DMD and NF1 group.

\begin{tabular}{|c|c|c|c|c|c|c|c|}
\hline \multirow[b]{3}{*}{ Questionnaires } & \multicolumn{3}{|c|}{$\operatorname{DMD}(n=38)$} & \multicolumn{3}{|c|}{$N F 1(n=38)$} & \multirow{3}{*}{$p$} \\
\hline & \multirow[t]{2}{*}{ Mean } & \multicolumn{2}{|c|}{$95 \% \mathrm{Cl}$} & \multirow[t]{2}{*}{ Mean } & \multicolumn{2}{|c|}{$95 \% \mathrm{Cl}$} & \\
\hline & & Lower & Upper & & Lower & Upper & \\
\hline CBCL int. & 61.2 & 56.3 & 66.1 & 51.6 & 46.8 & 56.4 & $.031^{*}$ \\
\hline CBCL ext. & 60.1 & 54.3 & 65.9 & 53.6 & 47.9 & 59.3 & .216 \\
\hline $\mathrm{CBCL}$ total & 61.8 & 56.6 & 67.0 & 57.2 & 52.2 & 62.3 & .328 \\
\hline TRF int. & 58.2 & 54.5 & 61.9 & 55.8 & 52.1 & 59.4 & .465 \\
\hline TRF ext. & 57.4 & 53.5 & 61.3 & 54.9 & 51.1 & 58.7 & .478 \\
\hline TRF total & 58.1 & 54.6 & 61.5 & 57.3 & 53.9 & 60.7 & .798 \\
\hline
\end{tabular}

Behavioural outcomes are means of z-scores corrected for the covariates age, the presence of comorbidities (learning, neurodevelopmental or behavioural disorders), use of stimulant medication (MPH), use of steroids, educational status of patients, and family history of learning and behavioural problems. $95 \% \mathrm{Cl}=95 \%$ Confidence Interval. $\mathrm{CBCL}$ int. $=\mathrm{CBCL}$ total internalizing problems scale score, $\mathrm{CBCL}$ ext. $=\mathrm{CBCL}$ total externalizing problems scale score, $C B C L$ total $=C B C L$ total problem score, $T R F$ int. $=T R F$ total internalizing problems scale score, TRF ext.=TRF total externalizing problems scale score, TRF total=TRF total problem score, DMD=Duchenne muscular dystrophy, NF1=Neurofibromatosis type $1 .{ }^{*} p<.05$ (two-sided).

\section{Discussion}

Cognitive- and behavioural problems are well known comorbidities in the neurogenetic disorders, DMD and NF1. A lack of protein expression in the brain may be responsible for the development of these brain-related comorbidities in both disorders. Genotypephenotype studies have investigated whether certain gene mutations result in specific 
and more severe phenotypes. In DMD, studies showed more severe cognitive and behavioural impairments in patients with mutations affecting the full-length and shorter brain isoforms, whereas in NF1 studies revealed more pronounced impairments in cognition, behaviour and learning in patients with microdeletions. Since in both neurogenetic disorders, different proteins are involved, we hypothesized that the cognitive and behavioural profiles of patients with DMD differ from patients with NF1. Surprisingly, no statistical significant differences were found between the groups on cognitive outcomes even after controlling for the covariates (age, comorbid diagnoses of patients (i.e. learning, neurodevelopmental, or behavioural disorders), use of stimulant medication (MPH), use of steroids, educational status of patients and family history of learning and behavioural problems). Results of reported behavioural problems by parents and teachers also displayed no significant differences between the DMD and NF1 group. Though, after controlling for covariates the multivariate results proved that there may be a significant difference between the groups on internalizing behavioural parent reports, reflecting more problems in de DMD group. This is contrary to previous literature showing that rates of depression, anxiety and psychosocial difficulties in DMD are not significantly worse due to disease characteristics compared to individuals with other chronic diseases. ${ }^{56-58}$

\section{Cognitive outcomes}

The intelligence quotients of our total DMD group were in general in accordance with previous data, with an overall mean FSIQ that was approximately one standard deviation below the population mean. ${ }^{16,59,60}$ No discrepancy between verbal IQ and performance IQ was found within our DMD group. This may likely be due to the large number of patients with distal mutations (55.3\%) in our study, of which is known that they exhibit lower intellectual abilities in general. ${ }^{60}$ Despite that our DMD group exhibit more difficulties on all intellectual tasks, their performances were not significantly lower compared to the NF1 group. However, the IQ distribution levels revealed that our DMD males predominantly fell within the low to low-average range, whereas the NF1 males performed low to normal. Higher rates of mental retardation ( $F S I Q<70$ ) have been described previously for DMD (30\%) than for NF1 (4-8\%), with most NF1 patients falling in the low-average to normal range. ${ }^{31,59}$ The IQ of our NF1 group was comparable to previous findings (IQ mean of 90$)^{4}$, which is on average comparable to the general population. Though, a variation in scores was noticed in our NF1 group, underscoring the heterogeneity in IQ in NF1. ${ }^{33}$ We found no correlation between the socialdemographic status of the patients and their IQ levels. 
Deficits in verbal span and working memory have long been documented as consistent cognitive features of DMD, but similar characteristics have been described for NF1. ${ }^{15,18,33,60-63}$ Within the present study both groups equally displayed lower sequential processing outcomes that were likely independent of IQ. Both especially exhibit difficulties in recalling information that increase in load in sequential order. On delayed memory and recognition memory, both groups performed comparable and approximately normal. These findings emphasize that males with DMD often display a limited verbal short-term memory and span capacity in the recall of specific sequence information, but not in consolidation or retrieval. ${ }^{61}$ Seeing the influence of limited verbal capacity on language development, attentional processes and learning it is important that the verbal memory problems are indicated at an early age. Particularly as it is shown that short-term memory and verbal span capacity are more powerful predictors for academic attainment of reading, writing and math than $10 .{ }^{60-62,64,65}$ Furthermore, patients with delays in verbal span capacity seem not to grow out their deficit $^{63}$, underscoring that early diagnosis and treatment of cognitive and academic problems should be part of regular clinical care of both neurogenetic disorders. ${ }^{66,67}$ In terms of psychological interventions clinicians may address tools that enhance or stimulate the learning of verbal auditory information, such as remedial teaching at school. ${ }^{66}$ Cognitive training for instance working memory training seems also a beneficial tool for children with short-term memory problems and learning disabilities $^{68,69}$, and it efficacy for patients with DMD and NF1 with comorbid learning disabilities should be investigated in future studies.

With respect to processing speed and visuospatial abilities (simultaneous processing), we found comparable outcomes for both groups. In DMD, most studies reported normal visuospatial abilities, but for NF1 the visuospatial disabilities are known cognitive features. ${ }^{15,31,70,71}$ A possible explanation for the absence of visuospatial disabilities in our NF1 group may depend on the less sensitive cognitive tasks that we used in current study. For NF1 regular clinical care at CNL, patients underwent various visuospatial and visuomotor tests, however certain tests that are part of the NF1 protocol (i.e. Rey-Osterrieth Complex Figure test) are not collected for patients with DMD. This limited our possibility in comparing visuospatial outcomes in which patients with NF1 display great deficits. ${ }^{4}$ Furthermore, we solely evaluated the cognitive profiles of males with NF1 and females were excluded, as the population of DMD predominantly involves men. However, gender in NF1 strongly influences phenotype expression and it is suggested that the clinical heterogeneity in NF1 likely results from an interplay between genomic determinants such as gender and neurofibromin functioning in specific tissue. ${ }^{72}$ This mechanism should be addressed in future studies 
investigating cognitive profiles in NF1, as most previous studies assessed cognition and behaviour in samples consisting of both sexes.

On sustained deficits in visual as well as auditory attention we found no differences between our groups. Within both neurogenetic disorders attention deficits are frequently reported, but to date most DMD and NF1 studies reference the prevalence rates of $A D(H) D$ as a marker of presence of attention problems, with little to no use of direct neurocognitive measures of attention. Only three previous DMD study used a cognitive attention task to estimate attention ${ }^{15,18,73}$, whereas two other studies used a processing speed task or verbal memory task. ${ }^{70,71}$ Studies addressing attention deficits solely based on ADHD prevalence rates should be interpreted with caution, because evidence is growing on the distinction between patients with ADHD with predominantly behavioural features (hyperactive/impulsive) and patients with the cognitive phenotype (inattention). ${ }^{33}$ Each type is suggested to have its own type of impairments, developmental trajectories and underlying neurobiology, which requires differentiation in diagnosis as well as in treatment. ${ }^{33}$

We noticed that $55.3 \%$ of our DMD group, had distal mutations abolishing the production of Dp140 and it is suggested that these males have more severe cognitive impairments. ${ }^{23,24,26}$ Additional post-hoc analyses checked whether the DMD males with mutations affecting Dp140 production (Dp140-, N=10), DMD males with intact Dp140 (Dp140+, N=21) and males with NF1 ( $N=38)$ differed. After applying Bonferroni correction we found a trend $(p=.60)$ for the group Dp140- indicating that these patients performed less well on processing speed compared to the other two groups (Dp140+ and NF1). It may be considered that in neurogenetic disorders not all cognitive functions are fully attributable to the genotype, but environmental and perinatal factors including maternal factors (e.g. stress, malnutrition, hypertension, substance (abuse) and fatal factors (hypoxia, low birth weight, prematurity) may be contributable and determinative for the phenotypes of patients as well. ${ }^{74}$

\section{Behavioural outcomes}

On average, males of both groups fell below the clinical cut off values on all syndrome scales, the broadband scales and the total scale scores of the CBCL and the TRF, representing that parents and teachers reported no significant elevated behavioural problems. More detailed analyses on abnormal ranges of the groups showed that parents and teachers of males with DMD more often reported aggressive behavioural problems. Prednisone is the standard prescription to stabilize muscle strength, extend 
ambulation and stand abilities in DMD and it is known that boys who take steroids exhibit more externalizing behavioural problems i.e. aggressive behaviour than boys taking no steroids. This may explain the higher rates of aggressive behaviour in our DMD group. However, results on the relation steroid use and higher incidences of externalizing behavioural problems are equivocal. ${ }^{58,75,76}$ Furthermore, DMD patients deal with physical milestones during the disease course, which may induce aggressive behaviour as well. For instance, our males were aged between 7-14 years and this is the age-range at which patients with DMD are confronted with loss of ambulation.

Within the NF1 group, parents more often reported difficulties in thinking and withdrawn, whereas teachers often reported social problems. It is interesting that the behavioural problems reported by parents and teachers of both groups differed in rates, with higher incidences reported by parents. In DMD, it is known that parent ratings are higher probably due to the parents perception of the magnitude of problems belonging to the illness and the increased parental stress resulting from difficult parent-child interactions. ${ }^{77,78}$ Our findings emphasize that screening of behavioural problems should be done by evaluating different perspectives (i.e. parents, teachers, patients and clinicians) on patients functioning. ${ }^{79}$ This is particularly important in neurogenetic disorders due to the presence of more than one cognitive or behavioural comorbidity and their overlap in symptoms. Furthermore, the $\mathrm{CBCL}$ may be no suitable instrument for screening behavioural problems, which we previously described in our systematic review. ${ }^{79}$ For clinicians it is important to know that results of the $\mathrm{CBCL}$ should be interpreted with caution and no definite diagnoses should be made solely on the basis of this instrument, as the symptom items of the $\mathrm{CBCL}$ subscales have no conceptual link with diagnostic criteria of behavioural disorders. ${ }^{80}$ In addition, insufficient psychometric properties have been found recently for the $\mathrm{CBCL}$ especially for patients with DMD. ${ }^{79}$ This may explain why some of the anomalies such as the lack of social problems in our DMD sample are not found, while $25 \%$ of them had a diagnosis of ASD. Due to our recent sensitivity findings of $C B C L$, the neuropsychological diagnostic work-up of patients with DMD is currently adapted in our Centre.

\section{Neurophysiology in relation to cognition and behaviour in DMD and NF1}

In both neurogenetic disorders, the affected proteins (i.e. dystrophin in DMD and neurofibromin in NF1) are expressed in a wide variety of nervous tissues including neurons and glial cells (e.g. astrocytes, oligodendrocytes) in the brain., ${ }^{7,0,30,81-83}$ A loss of the affected proteins result in functional and structural alterations of neurons and glial cells particularly located in corticostratial circuits and the hippocampus. ${ }^{7,30,81,82,84}$ For 
instance, by interacting with other components of the dystrophin-glycoprotein complex (DGC), such as syntrophin, the brain variant of the full-length dystrophin protein isoform $\left(D p 427_{B}\right)$ links to inhibitory $\gamma$-aminobutyric acid type $A\left(G A B A_{A}\right)$ receptors at the postsynaptic neural membrane. ${ }^{7,85} A$ lack of dystrophin results in a decreased density of $\mathrm{GABA}_{A}$ receptor clustering of receptor subunits at inhibitory synapses. ${ }^{84,85}$ Aberrant anchoring of $\mathrm{GABA}_{A}$ receptors causes an increased extrasynaptic expression of $\mathrm{GABA}_{\mathrm{A}}$ receptors, which triggers a disruption of calcium homeostasis and makes cells vulnerable to necrosis. ${ }^{86,87}$ Dystrophin deficiency also induces altered excitatory synapse functions and organizations i.e. abnormal enhanced NMDA receptor activation. ${ }^{10}$ In NF1, the decreased production of neurofibromin causes reduced Ras signalling molecule, leading to increased GABAenergic inhibition in the hippocampus due to impaired long-term potentiation. ${ }^{30,82}$ Furthermore, neurofibromin is localized at excitatory synapses postsynaptically where it interacts with the NMDA receptor. ${ }^{30,82}$ Overall, in both disorders neuronal alterations in $\mathrm{GABA}_{\mathrm{A}}$ and glutamate functions are found and these have been linked to the presence of neurocognitive deficits. ${ }^{7,10,30,81-83}$ It is tempting to speculate that due to the comparable neuronal defects we found no differences between the DMD and NF1 group. Though in DMD an increased excitation is described whereas in NF1 an increased inhibition is found, respectively. Further research should elucidate whether this dissimilarity effects the presence and severity of cognitive deficits. Another possible aetiology for the cognitive (and also learning) abnormalities in DMD and NF1 are glial dysfunctions (e.g. astrocyte abnormalities), but their contributory role needs further investigation. ${ }^{7,30}$ The proposed mechanisms that may underlie the cognitive phenotype in DMD are versatile. In addition, neuroimaging studies revealed individual variability in brain structures, networks, perfusion and metabolism. ${ }^{88}$ Future studies should link the cognitive outcomes to genetics (i.e. dystrophin isoform expression and neurophysiology) and neuroimaging, to better determine the factors involved in the presence and severity of the DMD cognitive phenotype. $^{88}$

\section{Limitations and future perspectives}

Unfortunately, not all collected data of the groups could be evaluated as other standard protocols have been used for regular clinical care for DMD and NF1 in our outpatient clinic. Therefore, academic skills for instance reading, writing and math that are often impaired in both groups were not assessed in current study. Due to the differences in neuropsychological batteries certain data were missing for which we applied stochastic regression imputation, but this does not take patients physical abilities into account. Furthermore, all participants of the present study were referred to the outpatient clinic 
Centre CNL, and these patients frequently have more (severe) learning, cognitive or behavioural than other patients with DMD or NF1, making our results likely less generalizable. Although, most prevalence rates of comorbid neurodevelopmental diagnoses and certain cognitive outcomes were in line with those reported by previous DMD and NF1 literature. In addition, we included participants aged 6-16 years to allow for the administration of the cognitive test and behavioural questionnaires, standardized for the Dutch population. However, cognitive and behavioural functions undergo major changes throughout childhood development, making mean group comparisons with large distributions of performances of young children (i.e. aged 6-7) and older children (15-16 years) difficult. Future studies should evaluate large samples size in which performances of different age groups can be evaluated. Nevertheless, our findings emphasize that comorbidities in cognition, behaviour and learning difficulties may arise in both neurogenetic disorders. Early cognitive and behavioural (re)evaluations are required and should be part of standards of care, in order to facilitate treatment as early as possible when necessary. Future longitudinal studies in DMD and NF1 should evaluate whether patients further grow in or out of their cognitive, behavioural and learning comorbidities. A nice addition to the NF1 literature would be to evaluate genotypes-phenotypes in severity and impact of cognitive, behavioural and learning comorbidities. These analyses could not be carried out in current study, since none of the NF1 patients had microdeletions.

\section{Conclusion}

The cognitive features of patients with DMD considerably overlap with those of male patients with NF1. It suggests that brain-related comorbidities in cognition are not only caused by gene mutations resulting in a lack of one specific protein, but also depend on other protein interactions and on neuronal and glial functional and structural alterations. Some differences in clinical features were noticed between the DMD and NF1 group, for instance the IQ levels of the DMD group were more distributed to the left compared to the NF1 group. With regard to behavioural features, aggressive behaviour was more often reported by parents and teachers of the DMD group, whereas in NF1 parents and teachers frequently reported problems with thinking, withdrawn and social behaviour. Clinicians should keep in mind that in both disorders one or more comorbidities may occur, that symptoms may overlap and that the severity of symptoms may variate between patients. This underscores that (re) evaluations and monitoring of cognitive development and behavioural functioning is required in both neurogenetic disorders. Possible cognitive or behavioural implications 
for treatment in both disorders could be for instance remedial teaching, cognitive (working memory) training, social training, psycho-education for patients, parents and teachers and neuropsychopharmacology.

\section{Acknowledgement}

The authors would like to thank the following people: prof. dr. E. Legius for his personal comments on genetic information on NF1, dr. M. Sinnema for the genetic information on microdeletions of the included patients with NF1, the patients of the Centre for Neurological Learning Disabilities that give consent to use their clinical data for scientific research purposes, and Erasmus Medical Centre for the collaboration in Neurofibromatosis 1 clinical care and scientific purposes. This research was funded by Spieren voor Spieren and Duchenne Parent Project NL. 


\section{References}

1. Bishop D. Genes, cognition, and communication: insights from neurodevelopmental disorders. Ann N Y Acad Sci 2009;1156:1-18.

2. Simon TJ. Clues to the foundations of numerical cognitive impairments: evidence from genetic disorders. Dev Neuropsychol 2011;36:788-805.

3. Snow WM, Anderson JE, Jakobson LS. Neuropsychological and neurobehavioral functioning in Duchenne muscular dystrophy: a review. Neurosci Biobehav Rev 2013;37:743-752.

4. Hyman SL, Shores A, North KN. The nature and frequency of cognitive deficits in children with neurofibromatosis type 1 . Neurology 2005;65:1037-1044.

5. Morel A, Demily C. Social cognition in children with neurogenetic syndromes: A literature review. Arch Pediatr 2017; 24:757-765.

6. Walter E, Mazaika P, Reiss A. Insights into brain development from neurogenetic syndromes: evidence from fragile $X$ syndrome, Williams syndrome, Turner syndrome and velocardiofacial syndrome. Neuroscience 2009;164:257-271.

7. Waite A, Brown SC, Blake DJ. The dystrophin-glycoprotein complex in brain development and disease. Trends Neurosci 2012;35:487-496.

8. Hoffman EP, Brown Jr RH, Kunkel LM. Dystrophin: the protein product of the Duchenne muscular dystrophy locus. Cell 1987;51:919-928.

9. Ehmsen J, Poon E, Davies K. The dystrophin-associated protein complex. J Cell Sci 2002;115:2801-2803.

10. Hendriksen RG, Hoogland G, Schipper S, Hendriksen JG, Vles JS, Aalbers MW. A possible role of dystrophin in neuronal excitability: a review of the current literature. Neurosci Biobehav Rev 2015;51: 255-262.

11. Doorenweerd N, Mahfouz A, van Putten M, et al. Timing and localization of human dystrophin isoform expression provide insights into the cognitive phenotype of Duchenne muscular dystrophy. Sci Rep 2017;7:12575.

12. Lidov $\mathrm{H}$, Byers $\mathrm{T}$, Kunkel L. The distribution of dystrophin in the murine central nervous system: an immunocytochemical study. Neuroscience 1993;54:167-187.

13. Chamberlain JS, Pearlman JA, Muzny DM, et al. Expression of the murine Duchenne muscular dystrophy gene in muscle and brain. Science 1988;239:1416-1418.

14. Banihani R, Smile S, Yoon G, et al. Cognitive and Neurobehavioral Profile in Boys With Duchenne Muscular Dystrophy. J Child Neurol 2015;30:1472-1482.

15. Cyrulnik SE, Fee RJ, Batchelder A, Kiefel J, Goldstein E, Hinton VJ. Cognitive and adaptive deficits in young children with Duchenne muscular dystrophy (DMD). J Int Neuropsychol Soc 2008;14:853-861.

16. Cotton S, Voudouris NJ, Greenwood KM. Intelligence and Duchenne muscular dystrophy: Full-Scale, Verbal, and Performance intelligence quotients. Dev Med Child Neurol 2001;43:497-501.

17. Wicksell RK, Kihlgren M, Melin L, Eeg-Olofsson O. Specific cognitive deficits are common in children with Duchenne muscular dystrophy. Dev Med Child Neurol 2004;46:154-159.

18. Wingeier K, Giger E, Strozzi S, et al. Neuropsychological impairments and the impact of dystrophin mutations on general cognitive functioning of patients with Duchenne muscular dystrophy. J Clin Neurosci 2011;18:90-95.

19. Pane $\mathrm{M}$, Lombardo $\mathrm{ME}$, Alfieri $\mathrm{P}$, et al. Attention deficit hyperactivity disorder and cognitive function in Duchenne muscular dystrophy: phenotype-genotype correlation. J Pediatr 2012;161:705-709. e701.

20. Hendriksen JG, Vles JS. Neuropsychiatric Disorders in Males With Duchenne Muscular Dystrophy: Frequency Rate of Attention-Deficit Hyperactivity Disorders (ADHD), Autism Spectrum Disorder, and Obsessive-Compulsive Disorder. J Child Neurol 2008;23(5):477-481.

21. Ricotti V, Mandy WP, Scoto M, et al. Neurodevelopmental, emotional, and behavioural problems in Duchenne muscular dystrophy in relation to underlying dystrophin gene mutations. Dev Med Child Neurol 2016;58:77-84.

22. Colombo $\mathrm{P}$, Nobile $\mathrm{M}$, Tesei $\mathrm{A}$, et al. Assessing mental health in boys with Duchenne muscular dystrophy: Emotional, behavioural and neurodevelopmental profile in an Italian clinical sample. Eur J Paediatr Neurol 2017;21(4):639-647. 
23. Chamova T, Guergueltcheva V, Raycheva M, et al. Association between loss of dp140 and cognitive impairment in duchenne and becker dystrophies. Balkan J Med Genet 2013;16:21-29.

24. Doorenweerd N, Straathof CS, Dumas EM, et al. Reduced cerebral gray matter and altered white matter in boys with Duchenne muscular dystrophy. Ann Neurol 2014;76:403-411.

25. Thangarajh M, Hendriksen J, McDermott M, Martens W, Hart K, Griggs R. Relationships between DMD mutations and neurodevelopment in dystrophinopathy (P5. 4-002). Neurology 2019;93(17): e1597-e1604.

26. Bardoni A, Felisari $G$, Sironi $M$, et al. Loss of Dp140 regulatory sequences is associated with cognitive impairment in dystrophinopathies. Neuromuscul Disord 2000;10:194-199.

27. Savar A, Cestari DM. Neurofibromatosis type I: genetics and clinical manifestations. Semin Ophthalmol 2008;23(1):45-51.

28. Rietman $A B$, Oostenbrink R, Bongers $S$, et al. Motor problems in children with neurofibromatosis type 1. J Neurodev Disord 2017;9:19.

29. Acosta MT, Bearden CE, Castellanos XF, et al. The Learning Disabilities Network (LeaDNet): using neurofibromatosis type 1 (NF1) as a paradigm for translational research. Am J Med Genet Part A 2012;158:2225-2232.

30. Gutmann DH. Neurofibromin in the brain. J Child Neurol 2002;17:592-601.

31. Schwetye KE, Gutmann DH. Cognitive and behavioral problems in children with neurofibromatosis type 1: challenges and future directions. Expert Rev Neurother 2014;14:1139-1152.

32. Hachon C, lannuzzi S, Chaix Y. Behavioural and cognitive phenotypes in children with neurofibromatosis type 1 (NF1): the link with the neurobiological level. Brain Dev 2011;33:52-61.

33. Templer AK, Titus JB, Gutmann DH. A neuropsychological perspective on attention problems in neurofibromatosis type 1. J Atten Disord 2013;17:489-496.

34. Torres Nupan MM, Velez Van Meerbeke A, Lopez Cabra CA, Herrera Gomez PM. Cognitive and Behavioral Disorders in Children with Neurofibromatosis Type 1. Front Pediatr 2017;5:227.

35. Hyman SL, Arthur E, North KN. Learning disabilities in children with neurofibromatosis type 1: subtypes, cognitive profile, and attention-deficit-hyperactivity disorder. Dev Med Child Neurol 2006;48:973-977.

36. Johnson H, Wiggs L, Stores G, Huson SM. Psychological disturbance and sleep disorders in children with neurofibromatosis type 1. Dev Med Child Neurol 2005;47:237-242.

37. Rietman $A B$, van der Vaart $T$, Plasschaert $E$, et al. Emotional and behavioral problems in children and adolescents with neurofibromatosis type 1. Am J Med Genet Part B Neuropsychiatr Genet 2018;177: 319-328.

38. North K, Hyman S, Barton B. Cognitive deficits in neurofibromatosis 1. J Child Neurol 2002;17:605-612.

39. Mautner V-F, Kluwe L, Thakker SD, Leark RA. Treatment of ADHD in neurofibromatosis type 1. Dev Med Child Neurol 2002;44:164-170.

40. Johnson NS, Saal HM, Lovell AM, Schorry EK. Social and emotional problems in children with neurofibromatosis type 1: evidence and proposed interventions. J Pediatr 1999;134:767-772.

41. Cnossen $\mathrm{MH}$, van der Est MN, Breuning $\mathrm{MH}$, et al. Deletions spanning the neurofibromatosis type 1 gene: implications for genotype-phenotype correlations in neurofibromatosis type 1? Hum Mutat 1997;9:458-464.

42. Leppig KA, Kaplan P, Viskochil D, Weaver M, Ortenberg J, Stephens K. Familial neurofibromatosis 1 microdeletions: cosegregation with distinct facial phenotype and early onset of cutaneous neurofibromata. Am J Med Genet 1997;73:197-204.

43. Boyd KP, Korf BR, Theos A. Neurofibromatosis type 1. J Am Acad Dermatol 2009;61:1-14.

44. Salman MS, Hossain S, Alqublan L, Bunge M, Rozovsky K. Cerebellar radiological abnormalities in children with neurofibromatosis type 1: part 1-clinical and neuroimaging findings. Cerebellum Ataxias 2018;5:14.

45. Roy A, Barbarot S, Charbonnier V, et al. Examining the frontal subcortical brain vulnerability hypothesis in children with neurofibromatosis type 1: are T2-weighted hyperintensities related to executive dysfunction? Neuropsychology 2015;29:473.

46. Verhage F. Intelligence and age: research among the Dutch between 7 and 77 years of age. Assen: Van Gorcum 1964.

47. Wechsler D. Manual for the Wechsler intelligence scale for children-(WISC-III). San Antonio, TX: Psychological Corporation 1991. 
48. Kaufman A. Manual for the Kaufman Assessment Battery for Children (K-ABC-II). Circle Pines (MN): American Guidance Service 2004.

49. Kalverboer A, Deelman B. De 15-woorden tests A en B:(een voorlopige handleiding)(15WT/VWT). Groningen: Academisch Ziekenhuis Groningen, afd Neuropsychologie 1986.

50. Vos P. Handleiding Bourdon-Vos test, 3e herziene uitgave. Lisse, Netherlands: Swets Test Publishers, 1998.

51. Manly T, Anderson V, Crawford J, George, M, , Underbjerg M, Robertson I. TEA-Ch: The test of everyday attention for children manual, Second Edition. 2016.

52. Achenbach T, Edelbrock C. Manual for the child behavior checklist (4-18) and 1991 Profile. Burlington: VT: University of Vermont, Department of Psychiatry, 1991.

53. Sterne JA, White IR, Carlin JB, et al. Multiple imputation for missing data in epidemiological and clinical research: potential and pitfalls. BMJ 2009;338:b2393.

54. Cohen J. A power primer. Psychol Bull 1992;112:155.

55. Field A. Discovering statistics using IBM SPSS statistics: Sage, 2013.

56. Fee RJ, Hinton VJ. Resilience in children diagnosed with a chronic neuromuscular disorder. Journal of developmental and behavioral pediatrics: JDBP 2011;32:644.

57. Darke J, Bushby K, Le Couteur A, McConachie H. Survey of behaviour problems in children with neuromuscular diseases. Eur J Paediatr Neurol 2006;10:129-134.

58. Hendriksen JG, Poysky JT, Schrans DG, Schouten EG, Aldenkamp AP, Vles JS. Psychosocial adjustment in males with Duchenne muscular dystrophy: psychometric properties and clinical utility of a parent-report questionnaire. J Pediatr Psychol 2009;34:69-78.

59. Cotton SM, Voudouris NJ, Greenwood KM. Association between intellectual functioning and age in children and young adults with Duchenne muscular dystrophy: further results from a meta-analysis. Dev Med Child Neurol 2005;47:257-265.

60. D'Angelo MG, Lorusso ML, Civati F, et al. Neurocognitive profiles in Duchenne muscular dystrophy and gene mutation site. Pediatr Neurol 2011;45:292-299.

61. Hinton V, Fee R, Goldstein E, De Vivo D. Verbal and memory skills in males with Duchenne muscular dystrophy. Dev Med Child Neurol 2007;49:123-128.

62. Leaffer EB, Fee RJ, Hinton VJ. Digit span performance in children with dystrophinopathy: a verbal span or working memory contribution? J Int Neuropsychol Soc 2016;22:777-784.

63. Hellebrekers DM, Doorenweerd N, Sweere DJ, et al. Longitudinal follow-up of verbal span and processing speed in Duchenne muscular dystrophy. Eur J Paediatr Neurol 2020;25:120-126.

64. Billard C, Gillet P, Barthez MA, Hommet C, Bertrand P. Reading ability and processing in Duchenne muscular dystrophy and spinal muscular atrophy. Dev Med Child Neurol 1998;40:12-20.

65. Alloway TP, Alloway RG. Investigating the predictive roles of working memory and IQ in academic attainment. J Exp Child Psychol 2010;106:20-29.

66. Birnkrant DJ, Bushby K, Bann CM, et al. Diagnosis and management of Duchenne muscular dystrophy, part 3: primary care, emergency management, psychosocial care, and transitions of care across the lifespan. Lancet Neurol 2018;17(5):445-455.

67. Ferner RE, Huson SM, Thomas N, et al. Guidelines for the diagnosis and management of individuals with neurofibromatosis 1. J Med Genet 2007;44:81-88.

68. Alloway T. Can interactive working memory training improving learning? Journal of Interactive Learn Res 2012;23:197-207.

69. Alloway TP, Bibile V, Lau G. Computerized working memory training: Can it lead to gains in cognitive skills in students? Computers Hum Behav 2013;29:632-638.

70. Hinton VJ, De Vivo DC, Nereo NE, Goldstein E, Stern Y. Selective deficits in verbal working memory associated with a known genetic etiology: the neuropsychological profile of Duchenne muscular dystrophy. J Int Neuropsychol Soc 2001;7:45-54.

71. Cotton S, Crowe SF, Voudouris N. Neuropsychological profile of Duchenne muscular dystrophy. Child Neuropsychol 1998;4:110-117.

72. Diggs-Andrews KA, Gutmann DH. Modeling cognitive dysfunction in neurofibromatosis-1. Trends Neurosci 2013;36:237-247.

73. Billard C, Gillet P, Signoret J, et al. Cognitive functions in Duchenne muscular dystrophy: a reappraisal and comparison with spinal muscular atrophy. J Neuromuscul Dis 1992;2:371-378. 
74. Faa G, Manchia M, Pintus R, Gerosa C, Marcialis MA, Fanos V. Fetal programming of neuropsychiatric disorders. Birth Defects Research Part C: Embryo Today: Reviews 2016;108:207-223.

75. Sienko S, Buckon C, Fowler E, et al. Prednisone and Deflazacort in Duchenne Muscular Dystrophy: Do They Play a Different Role in Child Behavior and Perceived Quality of Life? PLoS Curr 2016;8.

76. Hinton VJ, Nereo NE, Fee RJ, Cyrulnik SE. Social behavior problems in boys with Duchenne muscular dystrophy. Journal of developmental and behavioral pediatrics: JDBP 2006;27:470-476.

77. Bray P, Bundy AC, Ryan MM, North KN, Everett A. Health-related quality of life in boys with duchenne muscular dystrophy: Agreement between parents and their sons. J Child Neurol 2010;25:1188-1194.

78. Nereo NE, Fee RJ, Hinton VJ. Parental Stress in Mothers of Boys with Duchenne Muscular Dystrophy. J Pediatr Psychol 2003;28:473-484.

79. Hellebrekers DMJ, Lionarons JM, Faber CG, Klinkenberg S, Vles JSH, Hendriksen JGM. Instruments for the Assessment of Behavioral and Psychosocial Functioning in Duchenne and Becker Muscular Dystrophy; a Systematic Review of the Literature. J Pediatr Psychol 2019;44(10):1205-1223.

80. Goodman R, Scott S. Comparing the Strengths and Difficulties Questionnaire and the Child Behavior Checklist: is small beautiful? J Abnorm Child Psychol 1999;27:17-24.

81. Shilyansky C, Karlsgodt $\mathrm{KH}$, Cummings DM, et al. Neurofibromin regulates corticostriatal inhibitory networks during working memory performance. Proc Nat Acad Sci 2010;107:13141-13146.

82. Costa RM, Silva AJ. Molecular and cellular mechanisms underlying the cognitive deficits associated with neurofibromatosis 1. J Child Neurol 2002;17:622-626.

83. Patrakitkomjorn S, Kobayashi D, Morikawa T, et al. Neurofibromatosis type 1 (NF1) tumor suppressor, neurofibromin, regulates the neuronal differentiation of PC12 cells via its associating protein, CRMP-2. J Biol Chem 2008;283:9399-9413.

84. Anderson J, Morley J, Head S. Duchenne muscular dystrophy and brain function: INTECH Open Access Publisher, 2012.

85. Vaillend C, Perronnet C, Ros C, et al. Rescue of a dystrophin-like protein by exon skipping in vivo restores GABAA-receptor clustering in the hippocampus of the mdx mouse. Mol Ther 2010;18:16831688.

86. Kueh SL, Dempster J, Head SI, Morley JW. Reduced postsynaptic GABAA receptor number and enhanced gaboxadol induced change in holding currents in Purkinje cells of the dystrophin-deficient mdx mouse. Neurobiol Dis 2011;43:558-564.

87. Culligan K, Glover L, Dowling P, Ohlendieck K. Brain dystrophin-glycoprotein complex: persistent expression of $\beta$-dystroglycan, impaired oligomerization of Dp71 and up-regulation of utrophins in animal models of muscular dystrophy. BMC Cell Biol 2001;2:2.

88. Doorenweerd N. Combining genetics, neuropsychology and neuroimaging to improve understanding of brain involvement in Duchenne muscular dystrophy-a narrative review. Neuromusc Disord 2020;30(6): 437-442. 


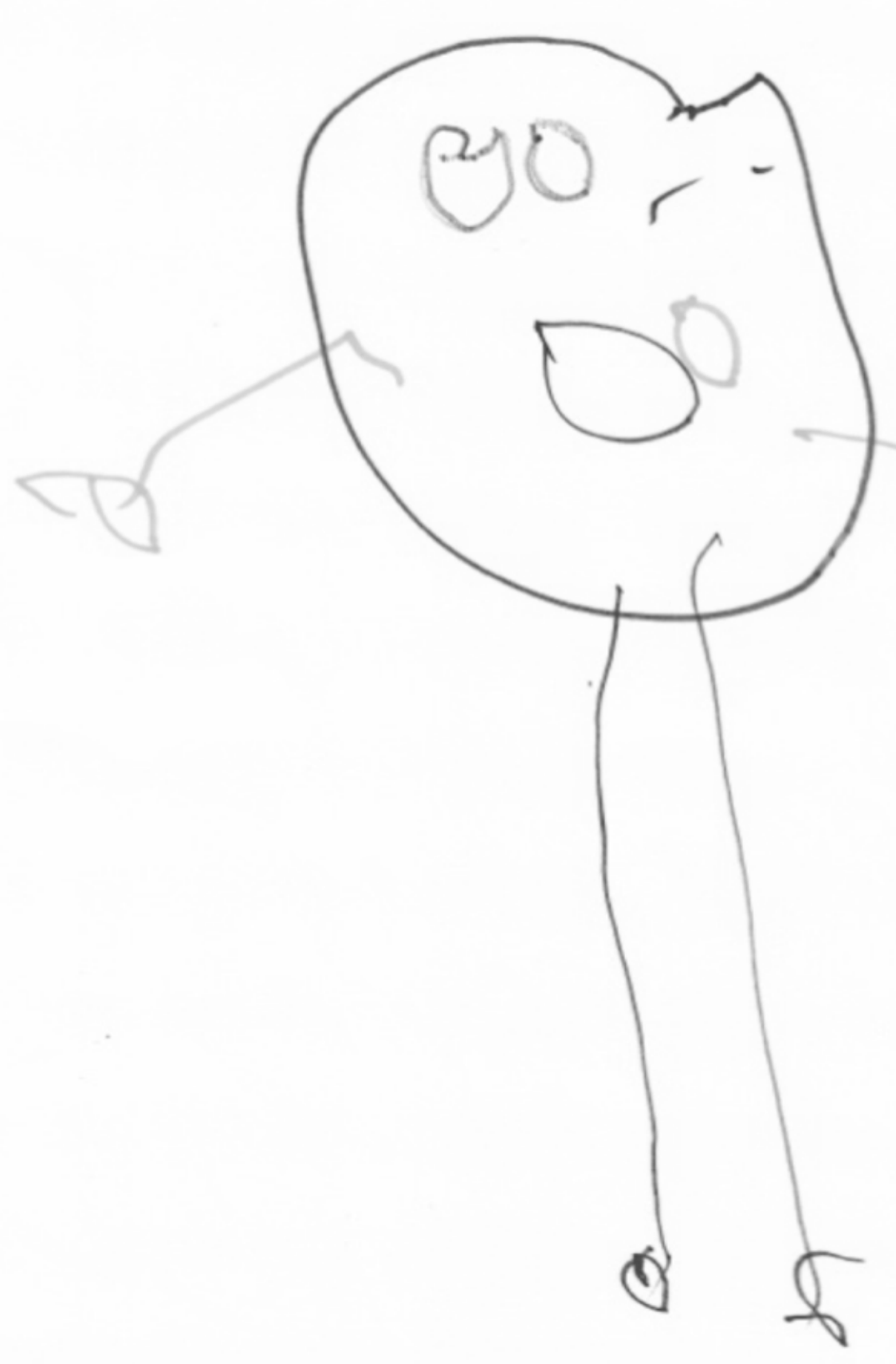




\section{Chapter \\ 3}

Longitudinal follow-up of verbal span and processing speed in Duchenne muscular dystrophy

Danique M.J. Hellebrekers, Nathalie Doorenweerd, D.J.J. Sweere, Sander M.J. van Kuijk, Annemieke M. Aartsma-Rus, Sylvia Klinkenberg, Johan S.H. Vles, Jos G.M. Hendriksen, European Journal of Paediatric Neurology 2020;28:120-126 


\section{Abstract}

Neurocognitive deficits are frequently described in Duchenne muscular dystrophy (DMD), but it is unknown how these progress over time. Our aim was to longitudinally assess verbal span capacity and information processing speed in DMD and to explore a genotype-phenotype relation. Verbal span and processing speed scores were available of 28 males with DMD on two time-points, with a mean time interval of 28.34 months $(S D=16.09)$. The cohort contained of six patients missing only dystrophin isoform Dp427, sixteen missing Dp427 and Dp140, and six were undeterminable. A lower verbal span capacity was found at the first and second assessment, whereas processing speed was normal at both time-points. Post-hoc analyses suggested lower scores on verbal span and processing speed for patients missing Dp427 and Dp140. In DMD, a developmental stagnation in verbal span capacity, irrespective of normal processing speed, is detected through longitudinal follow-up. This appears more pronounced in patients missing Dp427 and Dp140. 


\section{Introduction}

The X-linked neuromuscular disease, Duchenne muscular dystrophy (DMD) is characterized by severe and progressive muscle weakness due to mutations in the dystrophin-encoding $(D M D)$ gene. Mutations in the $D M D$ gene result in a lack of expression of the full-length dystrophin isoform (Dp427) in muscles (M) and the brain (B). ${ }^{1}$ In addition to the full-length isoform, shorter brain isoforms exist including Dp140, Dp71, and Dp40. A disturbed expression of the brain isoforms (Dp427B, Dp140, Dp71 + Dp40) may be related to the presence of neurocognitive problems in $D M D .^{1-6}$ It is proposed that the different isoforms are part of dystrophin-glycoprotein-like complexed (DGC-like) in either neurons or glia cells, depending on the dystrophin isoform. ${ }^{7}$ This complex can contain for example $\beta$-dystrobrevin, $\varepsilon$-sarcoglycan, dystroglycan, $\gamma$-syntrophins, neuronal nitrix oxide, GABA-A receptors or Acquaporin 4 receptors. ${ }^{1,7}$ However, little is known of the exact function of dystrophins in the brain, nor what happens to these DGC-like components in absence of dystrophin.

Previous studies have shown that males with DMD exhibit a specific neurocognitive profile in that the mean full-scale intelligence quotient (FSIQ) is approximately one standard deviation below the population mean. ${ }^{8}$ A lower verbal intelligence has in particular been described, whereas performance intelligence often seems preserved. ${ }^{7}$ In addition to the lower intellectual abilities, it is found that males with DMD exhibit cognitive impairments in verbal (working) memory, attention, executive functions, and academic achievement (reading, writing, and mathematics). ${ }^{9-13}$ The verbal memory deficits on "short-term memory" or "verbal span capacity" have consistently been found as core neurocognitive deficits in DMD and are expressed by a limited immediate recall of forward digits, words, stories, or sentence repetition. ${ }^{9,14-17}$ Interestingly, these verbal memory problems do not rely on the lower verbal intellectual abilities and are not caused by general impairments in language and memory. ${ }^{13,16,18} \mathrm{~A}$ decreased ability in retaining verbal information within short-term memory stores (i.e. the phonological loop) appears to be responsible for the poor academic achievement on reading, writing, and mathematics in males with DMD. ${ }^{11,14,16}$ In addition, their limited verbal capacity for retaining information in the phonological loop may explain their ineffectual proficiency on initial presentations of long statements or instructions. ${ }^{15,19}$ Despite the important role of verbal span capacity on academic skills and learning, it is unknown whether males with DMD further grow out of their verbal deficit, or whether it remains unchanged over time. The present study builds on previous evidence of limited verbal span capacity in DMD and provides the first longitudinal analyses on verbal span. An information processing speed task that does not rely on verbal memory was added as a 
control variable. As a result, the developmental pathways of both neurocognitive functions could be assessed. In addition, the present study further explores the relation between disturbed expression of brain isoform Dp140 and neurocognitive problems i.e. verbal span capacity and information processing speed in DMD.

\section{Materials and methods}

\section{Participants}

We included males with DMD who were seen for research purposes at the Leiden University Medical Centre (LUMC) or for clinical purposes at the outpatient clinical Centre for Neurological Learning disabilities (CNL) of Kempenhaeghe between 2010 and 2018. Males were included if they met the following criteria: (1) had a proven mutation of the $D M D$ gene which was previously genetically confirmed, (2) had neurocognitive data on verbal span capacity and information processing speed at two time points (i.e. first and second neurocognitive assessment) (3) were aged between six and sixteen years, and (4) had an adequate proficiency in Dutch. The age range of participants was chosen to allow for the administration of the cognitive test and behavioral questionnaires, standardized for the Dutch population. Exclusion criteria were: (1) a follow-up assessment within a period of six months or less and (2) physical immobility of upper extremities (hand and arm function) that could affect information processing speed scores. Ethical approval was granted by the local Medical Ethical Committees of both institutes and written informed consents were obtained.

\section{Study procedure}

The institutes LUMC and CNL used the same cognitive tasks to evaluate verbal span capacity and information processing speed during the first and second neurocognitive assessments. These tasks were administered by a researcher of LUMC (ND) and by psychologists of CNL, who were all trained by the same clinical neuropsychologist $(\mathrm{JH})$. The neurocognitive data and additional baseline participant characteristics i.e. demographic (age, educational level, estimated intelligence quotient score) and disease-related parameters (genetic mutation, wheelchair bound, comorbid neuropsychiatric and developmental diagnoses, and use of medication in particular methylphenidate and corticosteroids) were extracted from research files of LUMC or from the electronic patient files of CNL. Comorbidities extracted from the files were: attention-deficit (hyperactivity) disorders (ADHD or ADD), autism spectrum disorders (ASD), dyslexia, and dyscalculia. For this study, education was dichotomized in regular 
schooling or adapted education. All participants were further divided in three groups to explore associations between Dp140 expression and neurocognitive data on verbal span and information processing speed. Males with mutations not affecting Dp140 production (i.e. deletions or duplications upstream of intron 44) were considered Dp140+. ${ }^{20}$ If males had mutations abolishing Dp140 production (i.e. mutations corrupting the Dp140 promoter, the Dp140 translation start site or located downstream of exon 50 (the Dp140 ATG start-site is located in exon 51) they were considered Dp140-. ${ }^{20}$ Males with deletions or duplication breakpoints between intron 44 and exon 51 were assigned to a third group 'undefinable' and were left out of the subgroup comparison. ${ }^{20}$ In the current study, only one male had a mutation downstream of exon 56 affecting promoter site Dp116, and none had a mutation downstream of exon 63 affecting the promoter site Dp71.

\section{Neurocognitive assessment}

Intelligence quotient scores (IQ) were estimated by standardized scores (mean=100, $\mathrm{SD}=15$ ) of the Peabody Picture Vocabulary Test - third edition - Netherlands (PPVT-III$\mathrm{NL})^{21}$ during the first neurocognitive assessment. This non-motor test can be applied to estimate general intellectual functioning in a wide age range and has previously been used in DMD. ${ }^{10,19}$ Verbal span capacity was assessed by the forward Number Recall subtest of the Kaufman Assessment Battery for Children - second edition (KABC-II). ${ }^{22}$ Raw scores were converted to standardized scaled scores (mean=10, SD=3) based on age-adjusted norm data as specified in the test manual, with higher scores reflecting better performance. ${ }^{22}$ The Symbol Search subtest of the Wechsler Intelligence Scale for Children - third edition(WISC-II) ${ }^{23}$ was used to assess information processing speed. Raw scores were converted to standardized scaled scores (mean=10 and SD=3) based on age-adjusted norm data as specified in the test manual, with higher scores reflecting better performance. ${ }^{23}$ Current study only assessed the abovementioned cognitive tasks since the neurocognitive test battery of LUMC was administered for research purposes and consisted of a limited number of tasks, whereas the test batteries of CNL were based on individual request for help based on good clinical practice.

\section{Statistical methods}

Participant characteristics were represented as mean and standard deviation, or absolute number and proportion. One sample t-tests were used to compare results of the total sample to norm values. Differences between the following groups: (1) research population of LUMC and clinical population of CNL, and (2) the subgroups DMD_Dp140+ and DMD_Dp140- were tested, using the independent samples $t$-test, 
Mann-Whitney-U tests, and Fisher exact tests, as appropriate. Normal distributions were examined by visual inspection of histograms and normal probability plots. The raw and scaled scores of the 28 males with DMD were used to evaluate longitudinal data on verbal span capacity and information processing speed. Both scores were used to assess the developmental pathways i.e. unchanged raw scores and decreasing scaled scores (developmental stagnation), increasing raw scores and stable scaled scores (expected development), increasing raw and scaled scores (growing out of deficit), or decreasing raw and scaled scores (growing into deficit). Paired t-tests were used to test for differences between the first and second neurocognitive assessment within the total sample. To further evaluate individual clinical differences, z-scores of +2.0 SD or 2.0 SD were considered as significant changes and results were presented as absolute number and proportion. ${ }^{24}$ Furthermore, change (longitudinal) scores were computed by subtracting the scaled scores of the second assessment from the first assessment for each cognitive task. We used linear regression analyses to test whether age, individual differences in time between measurements in months, and use of stimulation medication had significant effects on the cognitive change scores. For post-hoc analyses, Mann-Whitney- $U$ tests were used to explore differences on neurocognitive data (scaled scores) between the subgroups Dp140+ and Dp140-. All statistical analyses were carried out using SPSS version 24.0 for MAC OS X. Results were considered significant if $p<.05$ (two-sided) for all analyses.

\section{Results}

\section{Participant characteristics}

Out of the total 75 number of patients identified, 28 DMD males met the inclusion/exclusion criteria of whom 11 participants of the LUMC research study and 17 patients of the CNL clinical population were included (see Figure 3.1). Differences in participant characteristics between the research and clinical population consisted of the research participants being significantly older $(p=.004)$ and with a significantly longer time between the first and second neurocognitive assessment $(p=.015$, see Supplementary Table S3.1). No significant differences were observed for any of the demographic characteristics such as educational level and estimated IQ scores, nor on disease related characteristics such as wheelchair bound, comorbid neuropsychiatric or developmental diagnoses, and use of corticosteroids or methylphenidate medication (see Supplementary Table S3.1). Patient characteristics of the included participants $(n=28)$ are displayed in Table 3.1. During the first neurocognitive assessment 13 males 
(46.4\%) of the total sample had no comorbid neuropsychiatric or developmental diagnoses, seven males (25\%) had a diagnosis of ADHD, two males (7.1\%) had ADD, two males (7.1\%) had ADD with dyslexia, three males (10.7\%) had ASD, and one male (3.6\%) had solely dyslexia. The mean time between the first and second neurocognitive assessment of the total sample was 28.34 months ( $S D=16.09$, range 7.33-57.76).

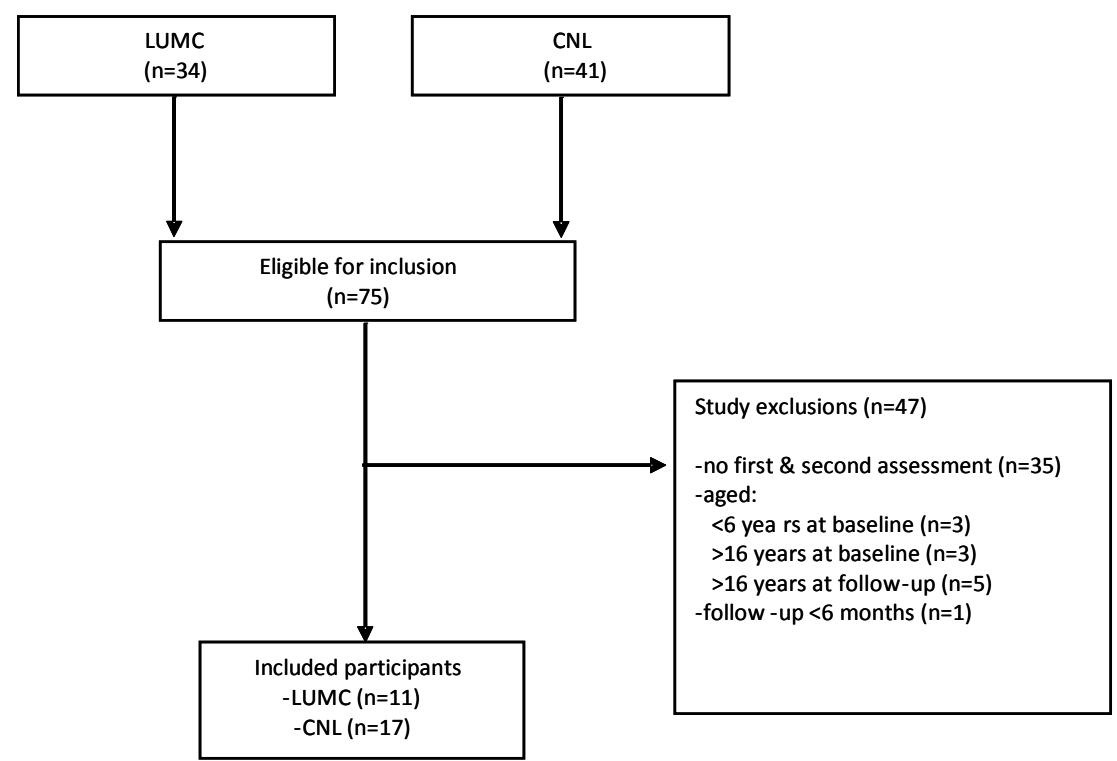

Figure 3.1 Flowchart of inclusion.

\section{Neurocognitive outcomes of DMD versus norm values}

The estimated IQ scores of the 28 males with DMD ranged between 71-129 and their IQ mean (103.7, SD=13.7; see Table 3.1) was not significantly different in relative to the population mean $(100, S D=15, p>.05)$. On verbal span capacity, a significantly lower mean scaled score was found when compared to the expected norm value of 10 on both the first (mean=7.6, SD=2.6, p<.001) and second neurocognitive assessment (mean=6.7, SD=3.1, $p<.001$ ). On information processing speed the total mean scaled score of the 28 males with DMD was not significantly different with respect to the norm value of 10 on their first (mean=9.1, SD=3.4, p>.05) or second neurocognitive assessment (mean=8.6, $\mathrm{SD}=4.3, p>.05)$. 
Table 3.1 Patient characteristics of the total DMD sample and of subgroups.

\begin{tabular}{|c|c|c|c|c|c|}
\hline & \multirow{2}{*}{$\begin{array}{l}\text { Total cohort } \\
\quad(n=28)\end{array}$} & \multicolumn{4}{|c|}{ Subgroups } \\
\hline & & $\begin{array}{c}\text { DMD_Dp140+ } \\
(n=6)\end{array}$ & $\begin{array}{c}\text { DMD_Dp140- } \\
(n=16)\end{array}$ & $\begin{array}{c}\text { Test statistic } \\
\text { value }\end{array}$ & $\begin{array}{c}p- \\
\text { value }\end{array}$ \\
\hline \multicolumn{6}{|l|}{ Demographic characteristics } \\
\hline Mean age in years (SD) & $8.7(2.2)$ & $8.28(1.2)$ & $9.05(2.4)$ & -0.442 & .658 \\
\hline \multicolumn{6}{|l|}{ Education (\%) } \\
\hline Regular school & $14(60.9)$ & $4(80)$ & $7(50)$ & 1.360 & .338 \\
\hline Adapted education & 9 (39.1) & $1(20)$ & $7(50)$ & & \\
\hline PPVT-III-NL score (n) & 20 & 4 & 11 & & \\
\hline Mean PPVT-III-NL (SD) & $103.7(13.7)$ & $108.0(7.7)$ & $99.8(15.3)$ & -0.654 & .513 \\
\hline \multicolumn{6}{|l|}{ Disease-related characteristics } \\
\hline Wheelchair dependency (\%) & $8(29.6)$ & $2(33.3)$ & $4(26.7)$ & 0.093 & 1.000 \\
\hline Comorbidity present at assessment (\%) & $15(53.6)$ & $4(66.7)$ & $8(50)$ & 0.489 & .646 \\
\hline \multicolumn{6}{|l|}{ Medication use (\%) } \\
\hline Corticosteroids & $23(82.1)$ & $6(100)$ & $14(87.5)$ & 0.825 & 1.000 \\
\hline Methylphenidate & $9(32.1)$ & $2(33.3)$ & $5(31.3)$ & 0.009 & 1.000 \\
\hline
\end{tabular}

Results are mean (SD) for continuous variables or frequency (\%). Test statistic values are $z$-values for continuous variables, and $\mathrm{X}^{2}$ or Fisher exact values for categorical variables, PPVT-III-NL=Peabody Picture Vocabulary Test- III-NL, unknown patient group is excluded from the subgroup analysis (n=6), DMD_Dp140+= males able to express Dp140, DMD_Dp140-=males unable to express Dp140.

\section{Longitudinal neurocognitive data in DMD}

\section{Verbal span}

The longitudinal raw scores on verbal span capacity of the 28 males with DMD showed no significant mean difference between the first and second neurocognitive assessment (see Table 3.2), even though an increase due to development would have been expected. See Figure 3.2 for visualization of individual trajectories of raw scores. In line with this, the longitudinal scaled scores showed a decrease over time on verbal span capacity (see Table 3.2), with a significant lower mean scaled score at the second neurocognitive assessment compared to the first neurocognitive assessment. Figure 3.3 depicts the visualization of the decreased scaled scores.

Table 3.2 Longitudinal neurocognitive data of the 28 males with DMD.

\begin{tabular}{|c|c|c|c|c|c|c|}
\hline & \multirow[b]{2}{*}{$\begin{array}{l}\text { First assessment } \\
\text { (mean; SD) }\end{array}$} & \multirow[b]{2}{*}{$\begin{array}{l}\text { Second assessment } \\
\text { (mean; SD) }\end{array}$} & \multirow[b]{2}{*}{$\begin{array}{c}\text { Test statistic } \\
\text { value }\end{array}$} & \multirow[b]{2}{*}{$p$-value } & \multicolumn{2}{|c|}{$95 \% \mathrm{Cl}$} \\
\hline & & & & & Lower & Upper \\
\hline \multicolumn{7}{|l|}{ Verbal span capacity } \\
\hline Raw score (range: 3-16) & $9.0(2.7)$ & $8.5(2.2)$ & 1.520 & .140 & -.200 & 1.343 \\
\hline Scaled score (range: 1-14) & $7.6(2.6)$ & $6.7(3.1)$ & -2.393 & $.024 *$ & -1.725 & -.132 \\
\hline \multicolumn{7}{|l|}{ Information processing speed } \\
\hline Mean raw score (range: 3-59) & $22.2(8.3)$ & $25.6(10.9)$ & 1.746 & .093 & -.605 & 7.420 \\
\hline Mean scaled score (range 1-16) & $9.3(3.3)$ & $8.6(4.3)$ & -0.895 & .379 & -2.44 & .961 \\
\hline
\end{tabular}

Mean and standard deviations, Test statistic values are t-values of the paired t-test, $95 \% \mathrm{Cl}=95 \%$ Confidence Interval, * $p<.05$ (two-sided) 


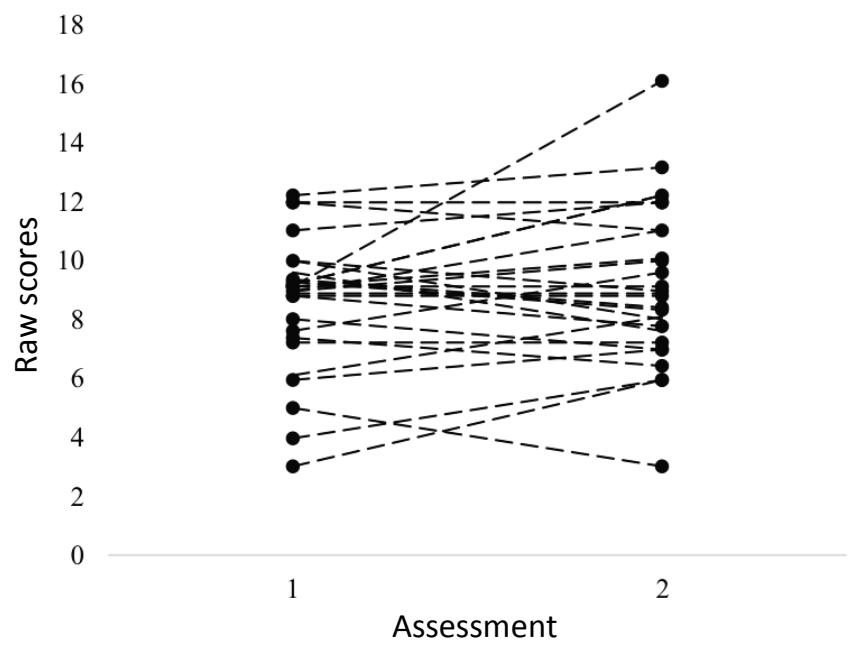

Figure 3.2 Raw scores of verbal span capacity.

15

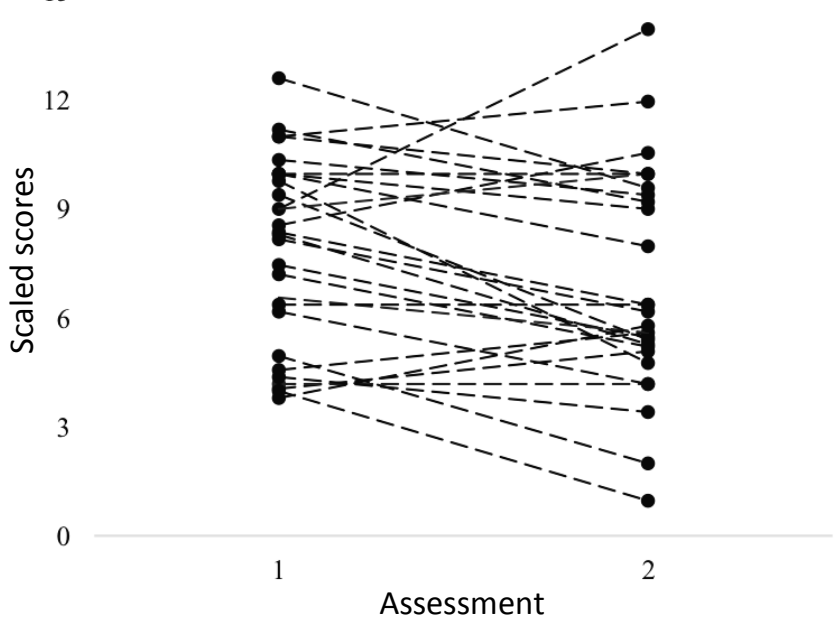

Figure 3.3 Scaled scores of verbal span capacity.

When assessing the scores of the 28 males individually, the z-scores of the second assessment did not decreased or increased more than 2SD compared to their first performance, indicating that none grown further in or out their possible span deficit. Regression analyses showed no significant effects of the covariates age, individual 
differences on time between assessment in months, or methylphenidate use on verbal span change score (see Table 3.3).

Table 3.3 Multiple regression analyses with verbal span change score as outcome.

\begin{tabular}{lcccccc}
\hline & & & & & \multicolumn{2}{c}{$95 \% \mathrm{Cl}$} \\
\cline { 6 - 7 } Variables & $\mathrm{B}$ & $\mathrm{SE}_{\mathrm{b}}$ & $\beta$ & $\mathrm{t}$-values & Lower & Upper \\
\hline Age & -0.174 & 0.190 & -0.184 & -0.915 & -0.565 & 0.218 \\
Individual differences in time & -0.015 & 0.029 & -0.120 & -0.536 & -0.074 & 0.044 \\
Methylphenidate use & -1.248 & 0.949 & -0.289 & -1.314 & -3.207 & 0.711 \\
\hline
\end{tabular}

$B=$ unstandardized regression coefficient, $S E b=$ standard error of the coefficient, $\beta=$ standardized coefficient, 95\% $\mathrm{Cl}=95 \%$ Confidence Interval, Age=age on baseline in years, Individual differences in time=individual differences in time in months between the first and second neurocognitive assessment. * $p<.05$

\section{Processing speed}

The longitudinal raw scores on information processing speed of the 28 males with DMD showed a slight but non-significant increase between the first and second neurocognitive assessment (see Table 3.2). See Figure 3.4 for visualization of raw scores. No significant differences were found on longitudinal scaled scores of information processing speed (see Table 3.2). See Figure 3.5 for visualization of scaled scores.

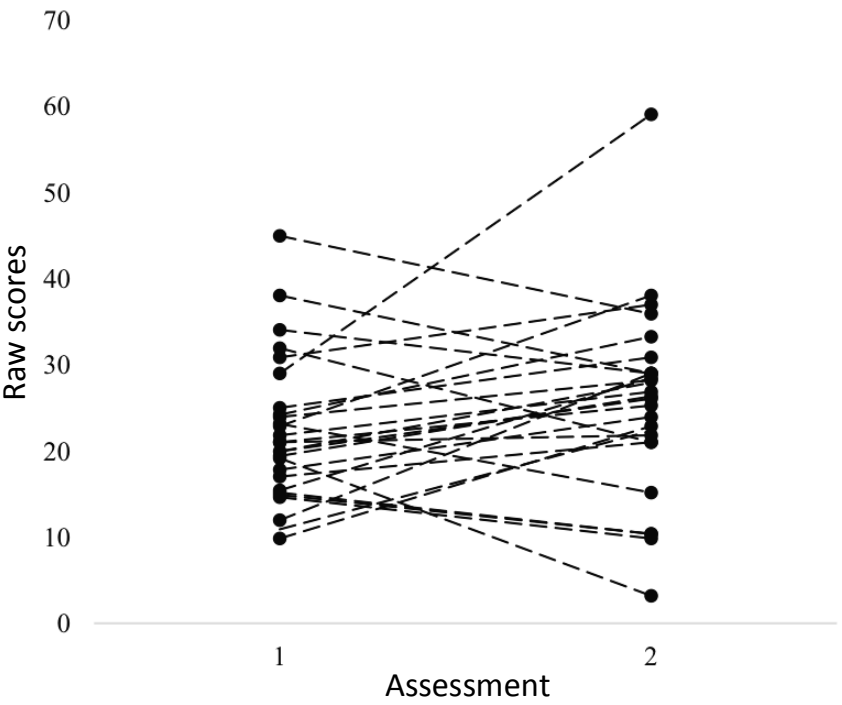

Figure 3.4 Raw scores of processing speed. 


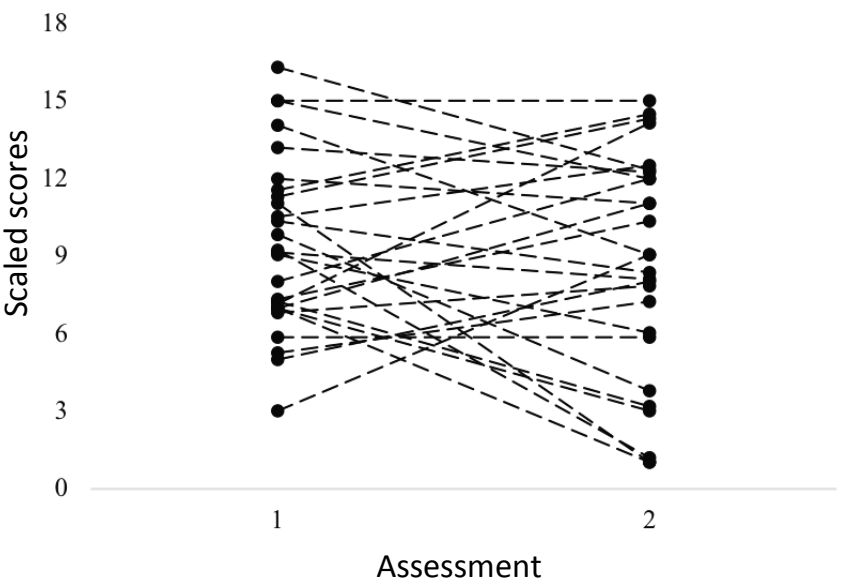

Figure 3.5 Scaled scored of processing speed.

No significant clinical differences ( $\geq 2$ SD) in z-scores were found for 21 (75\%) of males between their first and second assessment. Two males (7.1\%) showed a clinical relevant improvement at the second neurocognitive assessment. One of these two males had a comorbid diagnosis of ASS and the other male had no comorbid diagnosis. Four males (14.3\%) showed a clinical relevant decline at the second neurocognitive assessment. Two of these four males had a comorbid diagnosis of $A D(H) D$ and used methylphenidate medication on the second neurocognitive assessment. In addition, one of these four males had a comorbid diagnosis of ASD and one had no comorbid diagnosis. Regression analyses showed no significant effects of age, individual differences on time between assessment in months, and methylphenidate use on information processing speed change score when these variables were entered as covariates (see Table 3.4).

Table 3.4 Multiple regression analyses with information processing speed change score as outcome.

\begin{tabular}{lcccccc}
\hline & & & & & \multicolumn{2}{c}{$95 \% \mathrm{Cl}$} \\
\cline { 6 - 7 } Variables & $\mathrm{B}$ & $\mathrm{SE}_{\mathrm{b}}$ & $\beta$ & t-values & Lower & Upper \\
\hline Age & -0.737 & 0.391 & -0.379 & -1.546 & -1.546 & 0.072 \\
Individual differences in time & 0.049 & 0.060 & 0.185 & 0.813 & -0.075 & 0.172 \\
Methylphenidate use & -0.288 & 2.066 & -0.031 & -0.139 & -4.562 & 3.986 \\
\hline
\end{tabular}

$B=$ unstandardized regression coefficient, $S E b=$ standard error of the coefficient, $\beta=$ standardized coefficient, $95 \% \mathrm{Cl}=95 \%$ Confidence Interval, Age=age on baseline in years, Individual differences in time=individual differences in time in months between the first and second neurocognitive assessment. ${ }^{*} p<.05$ (two-sided). 


\section{Exploring the effect of Dp140 expression on neurocognition}

Of the 28 males with DMD, six were identified for group Dp140+ and sixteen were identified for group Dp140-. Six males were excluded from the subgroup analysis, because of the unpredictability of the mutation effect on Dp140 expression. Participant characteristics of the subgroups at the first neurocognitive assessment are presented in Table 3.1. No significant differences were found on demographic and disease related parameters between the Dp140+ subgroup and the Dp140- subgroup (see Table 3.1). The subgroup comparison on verbal span indicated significantly higher scores for group Dp140+ compared to group Dp140- at the first neurocognitive assessment $(p<.05)$, but not at the second neurocognitive assessment ( $p>.05$; see Table 3.5). The subgroup comparison on information processing speed indicated significantly higher scores for group Dp140+ compared to group Dp140- at both the first neurocognitive assessment $(p<.05)$ and second neurocognitive assessment $(p<.05$; see Table 3.5).

Table 3.5 Dp140 isoform expression and neurocognitive outcomes.

\begin{tabular}{lcccc}
\hline & $\begin{array}{c}\mathrm{Dp140-} \\
(\mathrm{N}=16)\end{array}$ & $\begin{array}{c}\mathrm{Dp} 140+ \\
(\mathrm{N}=6)\end{array}$ & $\begin{array}{c}\text { Test statistic } \\
\text { value }\end{array}$ & $p$-value \\
\hline $\begin{array}{l}\text { Verbal span capacity (median, range) } \\
\quad\end{array}$ & $7.0(4-10)$ & $10.0(6-12)$ & -2.194 & $.028^{*}$ \\
$\quad$ First assessment & $5.0(1-14)$ & $9.5(4-12)$ & -1.316 & .188 \\
$\quad$ Second assessment & & & \\
$\quad$ Information processing speed (median) & $8.0(3-15)$ & $12.5(4-16)$ & -2.082 & $.037^{*}$ \\
$\quad$ First assessment & $7.5(1-15)$ & $12.0(9-14)$ & -2.157 & $.031^{*}$ \\
$\quad$ Second assessment &
\end{tabular}

Results are median and range. Test statistic values are z-values. DMD_Dp140+=males able to express Dp140, DMD_Dp140-=males unable to express Dp140. * $p<.05$ (two-sided).

\section{Discussion}

The present study consisted of a convenience sample with both research and clinical data. Our results are consistent with the previously described impaired verbal span capacity among males with DMD., ${ }^{9,14}$ The 28 males with DMD of our sample had lower verbal span capacity on both the first and second neurocognitive assessment compared to norm values. On the contrary, performance on information processing speed was normal for both assessments compared to the norm values. With respect to the longitudinal evaluation, a developmental stagnation on verbal span capacity was found within our study. The developmental stagnation in verbal span was not influenced by factors such as age, individual differences in time between measurements in months, and use of methylphenidate. Despite our finding on the suggested non-progressive 
nature of verbal span capacity, it is important to note that a consistently reduced verbal span capacity is expected to severely impair academic development and learning in DMD. ${ }^{11,14,16-17}$ Verbal span is an important component of the working memory model proposed by Baddeley and Hitch, assuming that verbal information will be processed, stored and rehearsed by a system, referred as the phonological loop. ${ }^{25,26}$ Leaffer et al. (2016) reported that in addition to deficiencies within the phonological loop (i.e. holding verbal information in temporary storage), deficits in executive control cause an overall lower reading performance in DMD. ${ }^{16,27}$ Results of Leafer and colleagues (2016) and our results emphasize that cognitive (re-) evaluation with tasks involving the phonological loop as well as the central executive system are important for monitoring neurocognitive development, academic achievement, and learning in DMD. Clinicians should be aware of the possibility of cognitive developmental stagnations in DMD, particularly in domains involving verbal span capacity, academic skills, but also language acquisition. Thereby, it important for clinicians to adhere the clinical recommendations on cognitive re-evaluations that are documented in the updated standards of care for DMD. ${ }^{28}$ According to the standards of care, cognitive reevaluations should be performed every 2-3 years to facilitate early treatment in terms of remedial teaching and tools for teachers and parents when delays arise. ${ }^{28}$ For instance, when males exhibit a developmental stagnation in verbal span, tools as speaking simply, use short sentences, repeat verbal information more often, use visual stimuli as contextual cues, or associate pairs of words or digits to existing knowledge should be used at home and school to compensate and improve their ineffectual proficiency in retaining long and complex verbal information. ${ }^{15,26}$ A first cognitive evaluation should preferably be performed within the first year of diagnosis of DMD to establish a baseline ${ }^{28}$, but also to limit the influence of verbal span impairments on language acquisition. For realizing specific guidelines for DMD care on evaluation of cognition and learning, future research should longitudinally assess the development of working memory using complex dual tasks, crystallized and fluid intelligence as all contribute to academic performance. ${ }^{29,30}$

\section{Exploring the involvement of Dp140 expression on neurocognitive performance}

With respect to mutations affecting Dp140 expression, this study further explored the relation between loss of Dp140 and presence of neurocognitive deficits in DMD.,4,20 Even though the number of patients for this analysis is small, our subgroup analysis suggests that males with mutations affecting Dp140 expression performed more poorly on verbal span compared to males who can express Dp140. Additionally, processing 
speed was lower in males with disrupted Dp140 expression than in males with intact Dp140 expression. In four recent similar-aged population studies of males with DMD, mutations prohibiting Dp140 expression were also associated with neurocognitive deficits, in particular with verbal memory, even though these studies assessed this relation using other neurocognitive tasks or composed scores of cognitive tasks on intelligence, verbal (working) memory, and information processing speed. ${ }^{4,20,31}$ It is plausible that the phenotypes of males with DMD at least in part depend on the location of mutation and the additional disturbed expression of specific dystrophin isoforms within brain tissue. Although, further determination of the functions of the different DGC-like components is required to address the underlying mechanisms and to establish their role throughout brain development.

\section{Limitations}

Even though our sample size is appropriate for total group analyses, we acknowledge that our sample is too small to properly analyze differences between the subgroups, Dp140+ and Dp140-. However, our explorative results are relevant for clinical and research practices and of interest for future research. Secondly, the majority of our males (53.6\%) had a neurodevelopmental or psychiatric comorbidity that may have influenced our results on span development. However, it is previously proven that impairments in working memory and span also occur in males with DMD without neurodevelopmental or psychiatric disorders. ${ }^{32}$ Nonetheless, future longitudinal studies should perform subgroup analysis to evaluate the cognitive development of males with DMD without comorbidities, because no longitudinal assessment on their cognitive development exists. Thirdly, we had no control group, whereas other studies on DMD cognitive performance compared males with DMD to siblings or patients with other neuromuscular diseases. This limitation can be addressed in future longitudinal studies investigating DMD cognitive development, as this longitudinal study is the first step for a larger longitudinal follow-up study. Fourthly, some information on cognitive functioning (i.e. estimated verbal intellectual functioning; PPVT-III-NL scores) were not available of all participants due to a limited mental capacity of patients of CNL. Finally, we could not evaluate longitudinal changes of other cognitive domains since there were some differences between the neurocognitive assessment batteries of both institutes. Future research should replicate current longitudinal findings and include other neurocognitive tasks particularly tasks involving more complex working memory functions, intellectual abilities and academic skills (i.e. reading, math, spelling) with varying norm age-related scores, especially with a growing adult DMD population. 


\section{Conclusions}

We observed a developmental stagnation on verbal span capacity in males with DMD which remains present over time. A relatively stable growth curve has been suggested for information processing speed. Finally, DMD neurocognitive performance in verbal span capacity and information processing speed might be influenced by Dp140 loss, however this relation should be further explored.

\section{Acknowledgement}

The authors have stated that they had no interests which might be perceived as posing a conflict or bias. This work was supported by Spieren voor Spieren Foundation (JH.,SVS-15); Duchenne Parent Project NL (ND.,grant Brain imaging and cognition in Duchenne muscular dystrophy-2010); and Gratama Foundation University of Leiden (ND., grant number $\mathrm{nr}$ 10.13). The authors would like to thank all participants of Maastricht University Medical Center, the Leiden University Medical Centre and outpatient Centre of Neurological Learning disorders (CNL) of Kempenhaeghe, The Netherlands, and to Prof. dr. C.G. Faber, drs. J. Lionarons, prof. dr. J.J.G.M. Verschuuren, dr. E.H. Niks, and dr. H.E. Kan. 


\section{References}

1. Hendriksen RG, Hoogland G, Schipper S, et al. possible role of dystrophin in neuronal excitability: a review of the current literature. Neurosci Biobehav Rev 2015;51:255-262.

2. Doorenweerd N, Mahfouz A, van Putten M, et al. Timing and localization of human dystrophin isoform expression provide insights into the cognitive phenotype of Duchenne muscular dystrophy. Sci Rep 2017;7:12575.

3. Felisari G, Boneschi FM, Bardoni A, et al. Loss of Dp140 dystrophin isoform and intellectual impairment in Duchenne dystrophy. Neurol 2000;55:559-564.

4. Chamova T, Guergueltcheva V, Raycheva M, et al. Association between loss of dp140 and cognitive impairment in duchenne and becker dystrophies. Balkan J Med Genet 2013;16:21-29.

5. Banihani R, Smile S, Yoon G, et al. Cognitive and Neurobehavioral Profile in Boys With Duchenne Muscular Dystrophy. J Child Neurol 2015;30:1472-1482.

6. Ricotti V, Mandy WP, Scoto M, et al. Neurodevelopmental, emotional, and behavioural problems in Duchenne muscular dystrophy in relation to underlying dystrophin gene mutations. Dev Med Child Neurol 2016;58:77-84.

7. Waite A, Brown SC, Blake DJ. The dystrophin-glycoprotein complex in brain development and disease. Trends Neurosci 2012;35(8), 487-496.

8. Cotton SM, Voudouris NJ, Greenwood KM. Association between intellectual functioning and age in children and young adults with Duchenne muscular dystrophy: further results from a meta-analysis. Dev Med Child Neurol 2005;47:257-265.

9. Snow WM, Anderson JE, Jakobson LS. Neuropsychological and neurobehavioral functioning in Duchenne muscular dystrophy: a review. Neurosci Biobehav Rev 2013;37:743-752.

10. Cyrulnik SE, Fee RJ, Batchelder A, Kiefel J, Goldstein E, Hinton VJ. Cognitive and adaptive deficits in young children with Duchenne muscular dystrophy (DMD). J Int Neuropsychol Soc 2008;14:853-861.

11. Hinton VJ, De Vivo DC, Nereo NE, Goldstein E, Stern Y. Selective deficits in verbal working memory associated with a known genetic etiology: the neuropsychological profile of Duchenne muscular dystrophy. J Int Neuropsychol Soc 2001;7:45-54.

12. Billard C, Gillet P, Barthez MA, Hommet C, Bertrand P. Reading ability and processing in Duchenne muscular dystrophy and spinal muscular atrophy. Dev Med Child Neurol 1998;40:12-20.

13. Battini R, Chieffo D, Bulgheroni $S$, et al. Cognitive profile in Duchenne muscular dystrophy boys without intellectual disability: The role of executive functions. J Neuromuscul Dis 2018;28(2):122-128.

14. Hinton V, De Vivo D, Fee R, Goldstein E, Stern Y. Investigation of poor academic achievement in children with Duchenne muscular dystrophy. Learn Disabil Res Pract 2004;19:146-154.

15. Hinton V, Fee R, Goldstein E, De Vivo D. Verbal and memory skills in males with Duchenne muscular dystrophy. Dev Med Child Neurol 2007;49:123-128.

16. Leaffer EB, Fee RJ, Hinton VJ. Digit span performance in children with dystrophinopathy: a verbal span or working memory contribution? J Int Neuropsychol Soc 2016;22:777-784.

17. Mento G, Tarantino V, Bisiacchi PS. The neuropsychological profile of infantile Duchenne muscular dystrophy. Clin Neuropsychol 2011;25:1359-1377.

18. Hinton V, De Vivo D, Nereo N, Goldstein E, Stern Y. Poor verbal working memory across intellectual level in boys with Duchenne dystrophy. Neurol 2000;54:2127-2132.

19. Hinton VJ, Nereo NE, Fee RJ, Cyrulnik SE. Social behavior problems in boys with Duchenne muscular dystrophy. J Dev Behav Pediatr 2006;27:470-476.

20. Doorenweerd N, Straathof CS, Dumas EM, et al. Reduced cerebral gray matter and altered white matter in boys with Duchenne muscular dystrophy. Ann Neurol 2014;76:403-411.

21. Dunn L, Dunn L. Peabody Picture Vocabulary Test--Dutch Adaptation (PPVT-III-NL)(L. Schlichting, Trans.). Amsterdam: Harcourt Test Publishers, 2005.

22. Kaufman A. Manual for the Kaufman Assessment Battery for Children (K-ABC-II). Circle Pines (MN): American Guidance Service 2004.

23. Wechsler D. Manual for the Wechsler intelligence scale for children-(WISC-III). San Antonio, TX: Psychological Corporation 1991. 
24. Lezak MD, Howieson DB, Loring DW, Fischer JS. Neuropsychological assessment. Oxford University Press, USA; 2004.

25. Baddeley A. Working memory. Sci 1992;255:556-559.

26. Baddeley A. Working memory: looking back and looking forward. Nat Rev neuroscience 2003;4: 829-839.

27. Collette F, Van der Linden M. Brain imaging of the central executive component of working memory. Neurosci Biobehav Rev 2002;26:105-125.

28. Birnkrant DJ, Bushby K, Bann CM, et al. Diagnosis and management of Duchenne muscular dystrophy, part 3: primary care, emergency management, psychosocial care, and transitions of care across the lifespan. Lancet Neurol 2018;17(5):445-455.

29. Colom R, Escorial S, Shih PC, Privado J. Fluid intelligence, memory span, and temperament difficulties predict academic performance of young adolescents. Pers Individ Dif. 2007;42:1503-1514.

30. Alloway TP, Alloway RG. Investigating the predictive roles of working memory and IQ in academic attainment. J Exp Child Psychol. 2010;106:20-29.

31. Wingeier K, Giger E, Strozzi S, et al. Neuropsychological impairments and the impact of dystrophin mutations on general cognitive functioning of patients with Duchenne muscular dystrophy. J Clin Neurosci 2011;18:90-95.

32. Battini R, Chieffo D, Bulgheroni S, Piccini G, Pecini C, Lucibello S, Lenzi S, Moriconi F, Pane M, Astrea G, Baranello G. Cognitive profile in Duchenne muscular dystrophy boys without intellectual disability: The role of executive functions. J Neuromuscul Dis 2018;28:122-1228. 


\section{Supplemental data}

Table S3.1 Characteristics of the research and clinical sample.

\begin{tabular}{|c|c|c|c|}
\hline & $\begin{array}{c}\mathrm{CNL} \\
(n=17)\end{array}$ & $\begin{array}{l}\text { LUMC } \\
(n=11)\end{array}$ & $\begin{array}{c}\text { Test } \\
\text { Statistic value }\end{array}$ \\
\hline Mean time between measurements ${ }^{\#}$ (SD) & $22.3(16.5)$ & $37.7(10.2)$ & $-2.423^{*}$ \\
\hline \multicolumn{4}{|l|}{ Demographic characteristics } \\
\hline Mean age in years (SD) & $8.1(1.9)$ & $10.1(2.0)$ & $-2.917 * *$ \\
\hline \multicolumn{4}{|l|}{ Education (\%) } \\
\hline Regular school & $10(58.8)$ & $4(66.7)$ & 1.000 \\
\hline Adapted education & $7(41.2)$ & $2(33.3)$ & \\
\hline Mean PPVT-III-NL (SD) & $107.8(16.6)$ & $100.3(10.5)$ & -1.597 \\
\hline \multicolumn{4}{|l|}{ Disease-related characteristics } \\
\hline \multicolumn{4}{|l|}{ Type mutation (\%) } \\
\hline Dp140- & $11(78.6)$ & $5(62.5)$ & 0.624 \\
\hline Dp140+ & $3(21.4)$ & $3(37.5)$ & \\
\hline Wheelchair bound (\%) & $4(25.0)$ & $4(36.4)$ & 0.675 \\
\hline Comorbidity present at assessment (\%) & $11(64.7)$ & $5(45.5)$ & 0.441 \\
\hline \multicolumn{4}{|l|}{ Medication use (\%) } \\
\hline Corticosteroids & $14(82.4)$ & $9(81.8)$ & 1.000 \\
\hline Methylphenidate & $6(35.3)$ & $3(27.3)$ & 1.000 \\
\hline \multicolumn{4}{|l|}{ Neurocognitive outcomes } \\
\hline \multicolumn{4}{|l|}{ Verbal span capacity } \\
\hline First assessment & $7.8(2.3)$ & $7.4(3.0)$ & -0.380 \\
\hline Second assessment & $7.4(3.3)$ & $5.7(2.6)$ & -1.792 \\
\hline \multicolumn{4}{|l|}{ Information processing speed } \\
\hline First assessment & $9.3(3.4)$ & $8.8(3.7)$ & -0.285 \\
\hline Second assessment & $9.1(4.6)$ & $7.6(3.7)$ & -1.086 \\
\hline
\end{tabular}

Results are mean (SD) for continuous variables or frequency (\%). Test statistic values are $z$-values for continuous variables, and $\mathrm{X}^{2}$ or Fisher exact values for categorical variables, neurocognitive outcomes are scaled scores, DMD_Dp140+=males able to express Dp140, DMD_Dp140-=males unable to express Dp140. \# mean time in months * $p<.05$ (two-sided) ** $p<.01$ (two-sided). 


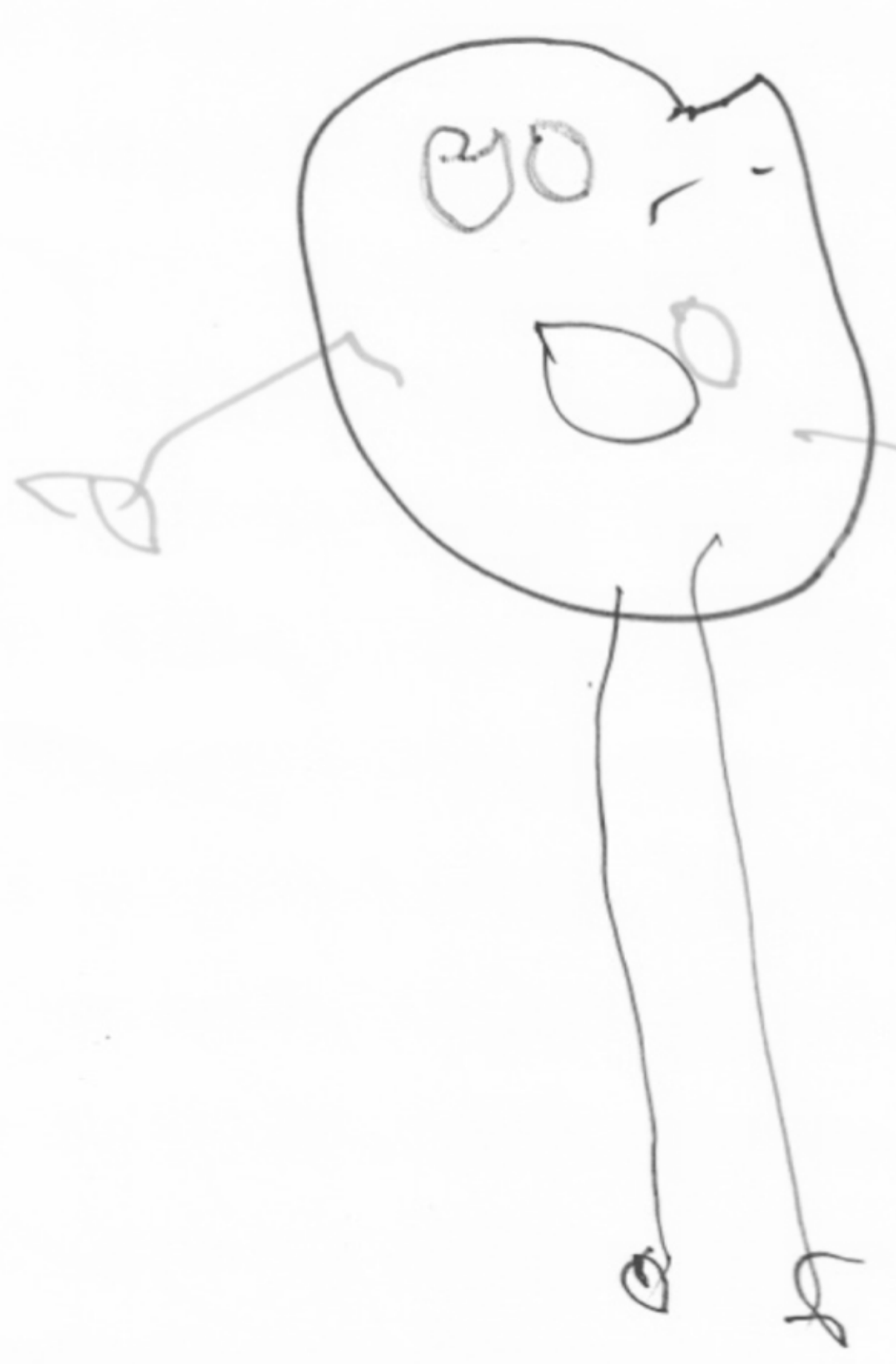




\section{Chapter 4}

\section{Minor cognitive impairments in adult males with}

Becker muscular dystrophy

Zaida Koeks ${ }^{*}$, Danique M.J. Hellebrekers', Nienke M. van de Velde, Iris Alleman, Petro Spitali, Hermien A. van Duyvenvoorde, Jan J.G.M. Verschuuren, Jos G.M. Hendriksen ${ }^{*}$, Erik H. Niks ${ }^{*}$

*contributed equally 
<smiles>Cc1ccccc1</smiles> 


\section{Chapter 5}

The neurocognitive and behavioural profiles of three

brothers with Becker muscular dystrophy

Danique M.J. Hellebrekers, Johan S.H. Vles, Sylvia Klinkenberg, Jos G.M. Hendriksen

Child Neurology Open 2020;7:1-5 


\section{Abstract}

Becker muscular dystrophy patients generally carry in-frame mutations in the dystrophin gene, allowing the production of partially functional dystrophin protein. The presence of cognitive and behavioural comorbidities and the relation with the location of mutations have been scarcely investigated in Becker. This case report describes the neurocognitive and behavioural profiles of three brothers with Becker carrying an inframe deletion of exons 45-48. The three cases underwent two consecutive neuropsychological assessments of which one assessment took place when they completed their primary education (age range of the cases: 11.2-12.1 years). Intellectual abilities were normal to high and all cases had difficulties with processing speed and math. The brothers differed in intellectual abilities, executive functions, working memory, attention and reading abilities. Variability in cognitive development was noted as well. This report suggests that cognitive and behavioural functions in Becker vary regardless of gene mutation and exposer to similar environmental factors. 


\section{Introduction}

Becker muscular dystrophy (BMD) is one of the recessive $x$-linked dystrophinopathies, caused by mutations in the dystrophin (DMD) gene. ${ }^{1}$ BMD mutations allow the production of internally deleted, but partially functional full-length dystrophin protein isoforms (Dp427) in various tissues including muscles (M) and the brain (B). ${ }^{1,2}$ The $(D M D)$ gene produces in addition to the full-length isoform, shorter isoforms, Dp260, Dp140, Dp116 and Dp71/Dp40. ${ }^{1-3}$ Dystrophin is a complex scaffold protein that modulates cellular homeostasis and multiple protein interactions. ${ }^{2}$ It provides structural stability to muscle fibres during contraction. ${ }^{2}$ The clinical severity of patients with BMD varies with some having a near normal functionality, whereas others lose the ability to walk during adolescence or early adulthood. ${ }^{1}$ In addition to skeletal muscle and heart pathology, patients with BMD may have neurodevelopmental-, behavioural comorbidities, learning disabilities and epilepsy. ${ }^{1,4-7}$ For instance, autism features, inattention/hyperactivity features, language-speech delays and difficulties in reading, spelling and arithmetic are found in BMD. ${ }^{1,4,6,7}$ It has been suggested that these comorbidities are related to a disturbed production of brain isoforms (i.e. Dp427 Dp140 and Dp71). ${ }^{8-9}$ However, this relation has been limitedly assessed in BMD. Only Bardoni and colleagues (2000) found intellectual disabilities in patients with BMD carrying mutations affecting $\mathrm{Dp} 427_{\mathrm{B}}$ and $\mathrm{Dp} 140$, but not in patients with only a disturbed expression of Dp427 ${ }_{\mathrm{B}}{ }^{8}$ A comparable association was assessed by Young et al.(2008), but in their BMD sample the intellectual abilities were not related to the mutation site. ${ }^{4}$ Additionally, the neurodevelopmental, behavioural and emotional problems in BMD are suggested to appear regardless of dystrophin gene mutation site. ${ }^{7}$ The current case report evaluates the neurocognitive and behavioural profiles of three brothers with BMD having a similar dystrophin mutation, to clarify whether a genotypephenotype relation may exist in BMD. We expect that the profiles of the brothers are similar

\section{Case report}

The reported cases were three brothers with BMD referred to the outpatient clinic of the Centre for Neurological Learning Disabilities (CNL), Kempenhaeghe, Heeze, The Netherlands. The diagnosis of BMD was previously confirmed by genetic testing between December 2011 and February 2012, which revealed an in-frame deletion of exon 45-48 (information on Dp140 promoter was not available). As part of their clinical care, each case received two consecutive neuropsychological assessments between 
2012 and 2018. The parents of the boys completed high school education and had no neuro-developmental-, behavioural comorbidities or learning difficulties. Written informed consents of patients and parents were obtained for current report.

\section{Neuropsychological assessment}

See Table 5.1 for an overview of tested neurocognitive domains and behavioural questionnaires.

Table 5.1 Overview of neurocognitive tests and behavioural questionnaires.

\begin{tabular}{ll}
\hline Domains & Tests \\
\hline $\begin{array}{l}\text { Intellectual functioning } \\
\text { general }\end{array}$ & WISC-III \\
verbal intelligence & \\
performance intelligence & \\
$\begin{array}{l}\text { Cognitive functions } \\
\text { working memory }\end{array}$ & \\
& \\
executive functioning & KABC-II sequential scale \\
attention & Digit span of WISC-III \\
speed of information processing & Mazes of WISC-III \\
visuospatial functioning & Bourdon Vos (continuous performance task) \\
visuomotor processing & Processing speed index of WISC-III \\
Academics & KABC-II simultaneous scale \\
reading & Beery VMI \\
mathematics & \\
Behavioural functioning & CB\&WL EMB-T50 (word reading) \\
& TTA (speeded arithmetic) \\
\hline
\end{tabular}

WISC-III=Wechsler Intelligence Scale for Children-third edition, KABC-II=Kaufmann Assessment Battery for Children-second edition, Beery VMI=Beery Visual-Motor Integration, TTA=Tempo Test Automatiseren, $\mathrm{CB} \& W L=$ Continu Benoemen \& Woordlezen, TTL=Tempo test Lezen, $\mathrm{CBCL}=\mathrm{Child}$ Behaviour Checklist, TRF=Teacher's Report Form.

The Dutch version of the Wechsler Intelligence Scale for Children-Third edition (WISCIII-NL) measured three WISC-III indexes (1) Verbal Comprehension (subtests information, similarities, vocabulary, comprehension, digit span, arithmetic), (2) Perceptual Organization (subtests picture completion, picture arrangement, block design, object assembly and mazes) and (3) Processing Speed (subtests coding and symbol search). Verbal Intelligence Quotient was obtained by adding the scaled scores of Verbal Comprehension index without digit span. Performance Intelligence Quotient (PIQ) was based on Perceptual Organization and Processing Speed indexes. The fullscale intelligence quotient (FSIQ) was obtained by adding scaled scores of all subtests. WISC-III subtest raw scores of the Digit span and Mazes were converted to age-related 
norm scores $(M=10, S D=3) .{ }^{10}$ The Kaufmann Assessment Battery for Children-second edition (KABC-II) evaluated (1) sequential processing based on the subtests Number Recall and Word Order and (2) simultaneous processing using the subtests Rover and Block Counting. ${ }^{11}$ Raw scores of the WISC-III (i.e. FSIQ, VIC, PIQ and Processing speed index) and KABC-II were converted to age-related norm scores ( $M=100, S D=15) .{ }^{10,11}$ Sustained attention was assessed using the speed and accuracy outcomes of the Bourdon Vos. ${ }^{12}$ Technical reading was evaluated by the Continu Benoemen \& Woordlezen (CB\&WL) using the EMTB-T50 score subtest. Raw scores reflect total number of words read correctly. ${ }^{13}$ Scores of the CB\&WL EMTB T50 and of the visuomotor processing task i.e. Beery-VMI were transformed to age-related norm scores $(M=10, S D=3) .{ }^{13,14}$ The Tempo Test Automatization (TTA) was used to evaluate the degree of automatization of mathematical facts. ${ }^{15}$ The TTA consists of four pages with 50 arithmetic problems including separate pages for addition, subtraction, multiplication and division problems. ${ }^{15}$ Raw scores are based on number of arithmetic problems answered correctly (range 0-50) and these were converted to age equivalent scores. ${ }^{15}$ The calculation of the age equivalent score is derived from a didactic (chronological) age score. This latest represents an expected score based on the number of months of arithmetic education a child has attended. At the end of primary regular education in the Netherlands, the didactic score reach it ceiling score of $60 .{ }^{15}$ The age equivalent score of TTA estimates the level of arithmetic functioning according to a patients didactic age. Behavioural functioning was screened using the Child Behaviour Checklist (CBCL) and Teacher Report Form (TRF). ${ }^{16,17}$ Both instruments evaluate behaviour based on eight syndrome scales. The syndrome scales can be used to calculate two broadband scale scores (i.e. internalizing and externalizing symptoms) and a total problem score. Raw scores were converted to T-scores $(M=50, S D=10)$. In line with the manual, a cut off-value of $T>63$ was applied to indicate clinical ranges of the broadband scales and the total problem score. ${ }^{16,17}$

\section{Demographic and disease-related characteristics}

The three included brothers had a genetic mutation involving a deletion of exons 45-48 of the DMD gene (see Table 5.2 for the age of the cases). None had hearing or vision problems, or used medication (i.e. steroids and stimulants) at time of neuropsychological testing. At first neuropsychological testing (TO), all cases followed regular education, were ambulant and had no formal neurodevelopmental or behavioural disorders. At second neuropsychological testing (T1; see Table 5.2 ), cases 1 and 2 continued with regular education, both remained ambulant and had no neurodevelopmental or behavioural disorders. Case 3 changed from special primary 
education for children with a physical handicap or learning disability to regular high school with a customized education plan. He was non-ambulant for long walking distances. Furthermore, based on the outcomes of his first assessment, case 3 was diagnosed with dyslexia by the neuropsychologist and child neurologist of the clinical team (JH, JV, SK).

Table 5.2 Neurocognitive and behavioural outcomes of the cases.

\begin{tabular}{|c|c|c|c|c|c|c|}
\hline \multirow[t]{2}{*}{ Variables } & \multicolumn{2}{|c|}{ Case 1} & \multicolumn{2}{|c|}{ Case 2} & \multicolumn{2}{|c|}{ Case 3} \\
\hline & TO & T1 & TO & $\mathrm{T} 1$ & TO & $\mathrm{T} 1$ \\
\hline Age at assessment in years & 7.7 & 11.8 & 11.3 & 14.5 & 9.3 & 12.1 \\
\hline \multicolumn{7}{|l|}{ Intellectual functioning } \\
\hline general & 1.3 & 0.5 & 1.5 & 1.5 & NA & 0.2 \\
\hline verbal index & 1.5 & 1.4 & 1.5 & 1.1 & NA & 0.4 \\
\hline performance index & 0.7 & -0.6 & 1.2 & 1.5 & NA & -0.7 \\
\hline \multicolumn{7}{|l|}{$\begin{array}{l}\text { Cognitive functions } \\
\text { working memory }\end{array}$} \\
\hline sequential & 0.4 & -0.6 & -0.2 & 0.2 & 0 & 0.2 \\
\hline digit span & -0.3 & 0.3 & -0.3 & 1.0 & NA & -1.0 \\
\hline executive functioning & 0.3 & -0.7 & 2.0 & 1.0 & NA & 0.3 \\
\hline \multicolumn{7}{|l|}{ attention } \\
\hline speed & -2.0 & -1.0 & -1.0 & -1.0 & 0.0 & -1.0 \\
\hline accuracy & -1.0 & 0.0 & 0.0 & 1.0 & -2.0 & 0.0 \\
\hline speed of information processing & 0.5 & -0.7 & -0.7 & -0.7 & 0.1 & -0.8 \\
\hline visuospatial & 1.5 & NA & 1.8 & 1.2 & 0.2 & 1.1 \\
\hline visuomotor & 0.0 & NA & 0.5 & -0.6 & 0.5 & -0.6 \\
\hline \multicolumn{7}{|l|}{$\begin{array}{l}\text { Academics } \\
\text { reading }\end{array}$} \\
\hline EMTB-T50 & 0.7 & 0.0 & NA & -1.3 & -1.7 & -2.3 \\
\hline speeded arithmetic ad/sub ${ }^{*}$ & 7 & 31 & 35 & 53 & 13 & 22 \\
\hline speeded arithmetic ad/sub/mu/di & - & 29 & 28 & 45 & $<15$ & 20 \\
\hline \multicolumn{7}{|l|}{ Behavioural functioning } \\
\hline \multicolumn{7}{|l|}{$\mathrm{CBCL}$} \\
\hline Internalizing problems & -0.9 & NA & 1.7 & 0.4 & 1.1 & 0.9 \\
\hline Externalizing problems & -1.7 & NA & 0.0 & -1.0 & -0.4 & -0.4 \\
\hline Total problems & -0.8 & NA & 0.4 & -0.5 & 0.5 & 0.2 \\
\hline \multicolumn{7}{|l|}{ TRF } \\
\hline Internalizing problems & 1.8 & NA & 0.6 & NA & 0.1 & NA \\
\hline Externalizing problems & 0.9 & NA & -0.9 & NA & -0.9 & NA \\
\hline Total problems & 0.6 & NA & -0.1 & NA & 0.0 & NA \\
\hline
\end{tabular}

Results are $z$-scores (mean $=0, S D=1$ ), except the speeded arithmetic scores. ${ }^{*}$ Are didactic age equivalent scores. $\mathrm{NA}=$ not available, EMTB-T50 = one minute reading test $\mathrm{T} 50$ score, speeded arithmetic $\mathrm{ad} / \mathrm{sub}=$ speeded arithmetic additions and subtractions total score, speeded ad/sub/mu/di=speeded arithmetic total score based on additions, subtractions, multiplications and division problems, $\mathrm{CBCL}=\mathrm{Child}$ Behaviour Checklist, TRF=Teacher report Form, T0=first neurocognitive testing, T1=second neurocognitive testing. Higher scores on the cognitive tests reflect better performances. Higher scores on the CBCL and TRF questionnaires reflect more behavioural problems. 
Neurocognitive and behavioral assessment at the end of primary education

All cases underwent neuropsychological testing at a similar age (11.3-12.1 years), which was at the end of primary education. Norm scores of the cognitive tests and behavioural questionnaires were transformed to $Z$-scores $(M=0, S D=1)$. Higher $z$-scores on the cognitive tests reflect better performances. Higher z-scores on the CBCL and TRF questionnaires reflect more behavioural problems. Results of cognitive testing showed that the three cases exhibited similar difficulties on speed (i.e. visual sustained attention speed and processing speed) and math (see Table 5.2; case 1 at T1, case 2 at T0 and case 3 at T1). The math outcomes of the three cases were lower than expected for their age, case 1 expected didactic score = 57 (at T1), case 2 expected didactic score $=51$ (at T0) and the expected didactic score of case 3 (at T1) could not be calculated because the didactic scores are not applicable to special elementary education. We also found differences in cognition. Case 1 performed lower on executive functioning compared to case 2 and 3. Case 3 had dyslexia and attention deficits, and this latest deficit caused high distractibility throughout testing. Additionally, his general and verbal intellectual abilities and working memory scores were lower compared to cases 1 and 2.

\section{Developmental profiles of the cases}

Table 5.2 displays the outcomes of the first and second testing of each case. The time between their first and second testing ranged from 2.8 to 4.1 years. Z-scores of +2.0 SD or -2.0 SD were considered as clinical significant changes. At T1, case 1 improved $(+1.0$ SD) on accuracy and speed of visual sustained attention (see Table 5.2). Furthermore, case 1 had more difficulties on visuospatial abilities (-1.1 SD), executive functioning (-1.4 SD), processing speed (-1.2 SD) and sequential processing (-1.2 SD; see Table 5.2). Other cognitive outcomes of T1 were stable compared to T0. The behavioural outcomes of case 1 could not be evaluated as the Child Behaviour Checklist ( $C B C L)$ and Teacher Report Form (TRF) were not completed at T1 (See Table 5.2). Case 2 improved on working memory (+1.0 SD) and accuracy of visual sustained attention (1.0 SD) at T1. Additionally, case 2 performed less on executive functioning (-1.0 SD) and visuomotor processing (-1.0 SD; see Table 5.2). The other outcomes remained comparable to T0, although his math outcomes were not applicable because he followed high school education at T1. With respect to the behavioural outcomes of case 2, we found an elevated score (1.7 SD) on CBCL internalizing subscale ( $T$-score $>63$ ) at $\mathrm{T0}$, but no longer at T1 (0.4 SD; see Table 5.2). Finally, case 3, had more difficulties with speed of visual sustained attention (-1.0 SD) and visuomotor processing (-1.1 SD) at T1 compared to T0 
(see Table 5.2). Other outcomes remained constant to T0 (see Table 5.2). No behavioural problems were noticed at both assessments of case 3 (see Table 5.2).

\section{Discussion}

The current study reports on a possible genotype-phenotype association in patients with BMD. This is the first case study on neurocognitive and behavioural profiles of three brothers with BMD with the same genetic dystrophin mutation, involving an inframe deletion of exon 45-48. We retrospectively evaluated cognitive and behavioural performances cross-sectionally and longitudinally. Our results showed comparable difficulties in math and processing speed among the cases, but we also noted differences in cognitive abilities. The general intellectual abilities (FSIQ) of case 3 were 1.3 SD lower compared to case 2. A comparable difference in FSIQ has been described by a previous study of Chamova and colleagues, who found a difference of 18 points (1.2 SD) in IQ (83 versus 65) among two brothers with BMD sharing a similar mutation defect of exon 45 to $53 .{ }^{18}$ Additionally, in our report, case 3 exhibited severe reading difficulties and attention problems, and this latest problem was expressed by a high internal distractibility throughout testing. A higher incidence of attention problems has previously been described for BMD. ${ }^{4}$ The prefrontal cortex which is involved in attentional processes is a region that is rich in dystrophin, which may suggest that abnormal brain dystrophin production contributes to the attention problems of dystrophinopathy patients. ${ }^{4,19-22}$ With respect to the development profiles of the cases, we noted non-significant clinical changes and some variability in development. Improvements were noticed for case 1 and 2 on accuracy of visual sustained attention, but both also displayed decreased performances in executive functions and processing speed. Furthermore, case 2 and 3 displayed more difficulties with visuomotor processing, longitudinally.

It was striking that we observed variability in cognitive and behavioural difficulties cross-sectionally and longitudinally, despite the fact that the cases had an identical dystrophin mutation, were tested at a similar age, and grew up in the same environment. We in particular found differences within intellectual abilities, working memory, attention and reading abilities. This may suggests that not only genetic and environmental factors induce interindividual variability in phenotypes. Other factors may modulate brain development as well. There is growing evidence on the fundamental role of maternal health factors on neurodevelopment of new-borns. ${ }^{23}$ Factors for instance as maternal stress, malnutrition, or prenatal exposure to toxic 
agents may also affect foetal brain development and induce altered brain structures and functions. However, the role of mechanism such as prenatal maternal stress on foetal brain development is not yet fully understood. ${ }^{23}$ Our results highlight that further research on cognitive and behavioural comorbidities and it development in the BMD population is necessary.

\section{Acknowledgments}

Special thanks to the patients and parents for their participation. Furthermore, the authors would like to thank prof. dr. A. Aartsma-Russ for providing feedback on the manuscript. This research was funded by Spieren voor Spieren and Duchenne Parent Project NL. 


\section{References}

1. Bushby KM, Gardner-Medwin DL, Nicholson LV, Johnson MA, Haggerty ID, Cleghorn NJ, Harris JB, et al. The clinical, genetic and dystrophin characteristics of Becker muscular dystrophy. J Neurol 1993;240: 105-112.

2. Mehler MF. Brain dystrophin, neurogenetics and mental retardation. Brain Res Rev. 2000;32:277-307.

3. Hendriksen RG, Hoogland G, Schipper S, Hendriksen JG, Vles JS, Aalbers MW. A possible role of dystrophin in neuronal excitability: a review of the current literature. Neurosci Biobehav Rev 2015;51: 255-262.

4. Young HK, Barton BA, Waisbren S, Portales Dale L, Ryan MM, Webster RI, et al. Cognitive and psychological profile of males with Becker muscular dystrophy. J Child Neurol 2008;23:155-162.

5. Goodwin F, Muntoni F, Dubowitz V. Epilepsy in Duchenne and Becker muscular dystrophies. Eur J Peaditrc Neurol 1997;1:115-119.

6. Mori-Yoshimura M, Mizuno Y, Yoshida S, Ishihara N, Minami N, Morimoto E, et al. Psychiatric and neurodevelopmental aspects of Becker muscular dystrophy. Neuromuscul Dis 2019;29:930-939.

7. Lambert JT, Darmahkasih AJ, Horn PS, Rybalsky I, Shellenbarger KC, Tian C, et al. Neurodevelopmental, behavioral, and emotional symptoms in Becker muscular dystrophy. Muscle Nerve 2019;61:156-162.

8. Bardoni A, Felisari G, Sironi M, Comi G, Lai M, Robotti M, et al. Loss of Dp140 regulatory sequences is associated with cognitive impairment in dystrophinopathies. Neuromuscul Dis 2000;10:194-199.

9. Daoud F, Angeard N, Demerre B, Martie I, Benyaou R, Leturcq F, et al. Analysis of Dp71 contribution in the severity of mental retardation through comparison of Duchenne and Becker patients differing by mutation consequences on Dp71 expression. Hum Mol Genet 2009;18: 779-3794.

10. Kort, W., M. Schittekatte, M. Bosmans, E. Compaan, G. Vermeir, and P. Verhaeghe. Wechsler Intelligence Scale for Children-III: Handleiding. Amsterdam: Pearson; 2005.

11. Kaufman AS. Manual for the Kaufman Assessment Battery for Children (K-ABC-II). Circle Pines, MN: American Guidance Service; 2004.

12. Vos P. Handleiding Bourdon-Vos test, 3e herziene uitgave.Lisse: Swets \& Zeitlinger; 1998.

13. Van den Bos, K. P., \& Lutje Spelberg, H. C. Continu Benoemen \& Woorden Lezen. Een test voor het diagnosticeren van taal-en leesstoornissen. Handleiding. Amsterdam; Boom test uitgevers; 2007.

14. Beery, K. E, Norman A,B., \& Beery, N.A. The Beery-Buktenica Developmental test of Visual-Motor Integration, $6^{\text {th }}$ edition. $\mathrm{VMI}$ administration, scoring, and teaching manual. Amsterdam: Pearson; 2010.

15. De Vos, T. Tempotest Automatiseren Handleiding en Verantwoording. Amsterdam: Boom test uitgevers; 2011.

16. Achenbach, T. M. Manual for the Child Behavior Checklist/4-18. Burlington: University of Vermont, Department of Psychiatry; 1991.

17. Achenbach, T. M., \& Edelbrock, C. S. Manual for the teacher report form and the child behavior profile. Burlington: University of Vermont, Department of Psychiatry; 1986.

18. Chamova T, Guergueltcheva V, Raycheva M, Todorov T, Genova J, Bichev S, et al. Association between loss of dp140 and cognitive impairment in duchenne and becker dystrophies. Balkan J Med Genet 2013; 16:21-29.

19. Pane M, Lombardo ME, Alfieri P, D'Amico A, Bianco F, Vasco G, et al. Attention deficit hyperactivity disorder and cognitive function in Duchenne muscular dystrophy: phenotype-genotype correlation. J Pediatr 2012;161:705-709.

20. Ricotti V, Mandy WP, Scoto M, Pane M, Deconinck N, Messina S, et al. Neurodevelopmental, emotional, and behavioural problems in Duchenne muscular dystrophy in relation to underlying dystrophin gene mutations. Dev Med Child Neurol 2016;58:77-84.

21. Doorenweerd N, Mahfouz A, van Putten M, Kaliyaperumal R, t'Hoen PA, Hendriksen JG, et al. Timing and localization of human dystrophin isoform expression provide insights into the cognitive phenotype of Duchenne muscular dystrophy. Sci Rep 2017;7:1-2.

22. Arnsten AF, Lombroso PJ. Genetics of childhood disorders: XVIII. ADHD, Part 2: Norepinephrine has a critical modulatory influence on prefrontal cortical function. J Am Acad Child Adolesc Psychiatry 2000; 39:1201-1203. 
23. Faa G, Manchia M, Pintus R, Gerosa C, Marcialis MA, Fanos V. Fetal programming of neuropsychiatric disorders. Birth Defects Res C Embryo Today 2016;108:207-223. 
<smiles>Cc1ccccc1</smiles> 


\section{Chapter 6}

Instruments for the assessment of behavioral and psychosocial functioning in Duchenne and Becker muscular dystrophy; a systematic review of the literature

Danique M.J. Hellebrekers, Judith M. Lionarons, C.G. Faber, Sylvia Klinkenberg, Johan S.H. Vles, Jos G.M. Hendriksen Journal of Pediatric Psychology 2019;44(10), 1205-1223 


\section{Abstract}

This systematic review aims to provide an overview of instruments used to assess behavioural and psychosocial functioning of patients with Duchenne and Becker muscular dystrophy, as well as to review the psychometric properties and applicability of these instruments. Five databases (Embase, Psyc.info, ERIC, Pubmed/Medline, and Cochrane) were searched from inception to June, 2018. Potential articles were rated by two independent reviewers. A predefined PROSPERO form (CRD42017074518) was used to extract data from included articles. Sixty-one instruments were used in 54 studies. The Child Behaviour Checklist is commonly used, but it lacks disease specific psychometric information. Sixteen instruments that contained disease specific psychometric information were included for final evaluation. The results displayed three instruments can be appropriate for screening of psychosocial problems: the Psychosocial Adjustment and Role Skills Scale $3^{\text {rd }}$ edition, the Paediatric Quality of Life Inventory Generic module, and the Life Satisfaction Index for Adolescents with Duchenne muscular dystrophy. Appropriate instruments for screening of behavioural problems may be: the Strengths and Difficulties Questionnaire, the Generalized Anxiety Disorder-7 item questionnaire, and the Patient Health Questionnaire. Further research on psychometric properties of the abovementioned screening instruments is crucial to ascertain a gold standard for clinical and research purposes. Meanwhile, for definite diagnostics purposes we recommend a multi-method, multi-source, multi-setting assessment in this high-risk population. 


\section{Introduction}

Duchenne muscular dystrophy (DMD) and Becker muscular dystrophy (BMD) are allelic disorders covering the spectrum of X-linked dystrophinopathies, and are further mentioned as the dystrophinopathy population. Both disorders are caused by mutations in the $D M D$ gene, that encodes multiple dystrophin isoforms in various tissues (i.e. muscles and the brain). ${ }^{1-3}$ Depending on mutation location one or more dystrophin protein isoforms are absent in DMD, resulting in progressive muscle wasting. ${ }^{2,3}$ By contrast, in $\mathrm{BMD}$, gene mutations cause an open reading frame with a milder and more variable phenotype. ${ }^{4}$ In addition to the myopathy, behavioural disorders are common in the dystrophinopathy population. ${ }^{5-7}$ High prevalence rates of autism spectrum disorders (ASD; up to 21\%), attention-deficit hyperactivity disorders (ADHD; up to 32\%), anxiety and depression (up to 27\%), oppositional defiant disorders (ODD; 15\%), and obsessive compulsive disorders (OCD; up to $5 \%$ ) have been described in DMD compared to the general. ${ }^{5,8-12}$ Additionally, impaired psychosocial functioning has been reported in. ${ }^{13,14}$ Three previous BMD studies also investigated the presence of behavioural disorders, ${ }^{4,7,15}$ but only one study found increased prevalence rates of attention problems (33\%), ASD (8.3\%), social problems (33\%), and withdrawn behaviour (33\%) in BMD compared to the general population. ${ }^{7}$ Current evidence suggests that these behavioural disorders are partially caused by neurobiological processes and relates them to impaired or altered expression of brain dystrophin isoforms. A relation between the absence of brain dystrophin isoforms and behavioural comorbidities such as attention-deficit hyperactivity- and autism spectrum disorders has been described in patients with $\mathrm{DMD}^{1,8,10,11}$ There is no research available yet on possible relations between partially functional dystrophin production in the brain and behavioural comorbidities in BMD. Research on dystrophin-associated behavioural comorbidities remains limited for both disorders and no clear explanation exists for the different rates in comorbidities between DMD and BMD. It is suggested that the discrepancy might be explained by the difference in expression of brain dystrophin isoforms in DMD versus $B M D$, but this needs further investigation.

With the increased life expectancy nowadays due to changes in medical care (i.e. improved ventilation assistance), the dystrophinopathy population is confronted with long-lasting or new mental health issues that negatively influence their quality of life. ${ }^{16 .}$ Recent standards of care for DMD emphasize that adequate and routine behavioural and psychosocial assessment is crucial, ${ }^{16}$ though current literature lacks an overview of available and adequate instruments. Nonetheless, the updated standards of care for DMD recommend four instruments: the Strengths and Difficulties Questionnaire (SDQ), 
the Patient Health Questionnaire (PHQ-9), the Generalized Anxiety disorder 7-item scale (GAD-7), and the Personal Adjustment and Role Skills Scale-III (PARS-III) for DMD. ${ }^{16}$ However, disease-specific psychometric information is unknown for the SDQ, the PHQ-9, and the GAD-7. The suitability of the PARS-III for DMD care has been shown by only one previous study. ${ }^{17}$

Recently, improvements in screening and diagnostics of behavioural and psychosocial problems have received more attention in this high-risk aging population. Several studies have tried to improve the assessment of behavioural and psychosocial problems by using (1) the Diagnostic and Statistical Manual of Mental Disorders criteria, (DSM) ${ }^{8}$, (2) more than one instrument, (e.g. informant-ratings in addition to structured clinical interviews), ${ }^{8,11,18}$, or (3) instruments with items relevant to dystrophinopathy (e.g. Muscular Dystrophy Child Health Index of Life with Disabilities questionnaire for children with DMD, MDCHILD). ${ }^{19}$ However, most studies have used instruments with poor or unevaluated psychometric properties or instruments that contain items unsuited for patients with motor impairments. Therefore, the present systematic review aims to provide an overview of instruments used to assess behavioural and psychosocial functioning, and evaluates their psychometric properties and applicability for the dystrophinopathy population.

\section{Methods}

\section{Search strategy and selection criteria}

This systematic review was conducted according to the Preferred Reporting Items for Systematic Reviews and Meta-Analyses (PRISMA) statement ${ }^{20}$ and predefined PROSPERO protocol (CRD42017074518). Databases (EMBASE, Psyc.info, ERIC, PUBMED/Medline, and Cochrane) were searched from inception to July 2017 and updated to June, 19, 2018. The search strategy is presented in Supplementary Table S6.1. To be eligible for inclusion, studies had to (a) include males with dystrophinopathy (i.e. DMD or BMD), (b) examine the psychometric properties of behavioural and psychosocial instruments, (c) be available in English, Dutch or German, and (4) be longitudinal or cross-sectional design studies. Case report studies $(n<10)$, non-peer reviewed articles, abstracts from scientific meetings, intervention studies or studies using an instrument that consisted of one subscale or one item of a questionnaire were excluded. 
Two reviewers (D.H.: psychologist and J.L.: Doctor of Medicine, both having clinical experience with patients with DMD and BMD) independently screened titles and abstracts manually for potential inclusion, using the search terms of Supplementary Table S6.1. Full-texts of articles were retrieved when inclusion could not be based on title and abstract. Cohen's kappa was used to measure the inter-rater reliability between the two independent reviewers (D.H. and J.L.). If no consensus was reached between the two reviewers, a third independent reviewer (J.H. psychologist with clinical experience in DMD and BMD) examined whether the article fulfilled the inclusion criteria. After selection based on title and abstract, one reviewer (D.H.) screened the full-text of the selected articles to assess whether the article could be included for final evaluation (i.e. data extraction). Doubtful articles were examined by a second independent reviewer (J.H.). Furthermore, reference lists of the selected fulltext articles were screened to identify whether additional articles had to be included for review.

\section{Data collection and extraction}

One reviewer (D.H.) extracted data from included articles for final evaluation using a predefined form (PROSPERO: CRD42017074518). The following data were extracted: (1) authors and year of publication, (2) demographic characteristics (e.g. age), (3) inand exclusion criteria if specified, (4) study design, (5) instruments used to assess behavioural or psychosocial functioning, (6) results of psychometric properties and applicability of instruments, and (7) study limitations and main conclusion.

\section{Classification of method of assessment}

Selected articles were classified according to methods of assessment (Table 6.2): clinical observation ratings, (semi-) structured clinical interviews, informant-ratings, and selfratings. During clinical observations, a clinician rates the extent of behavioural or psychosocial problems based on clinical judgement. Structured clinical interview instruments are designed to acquire both quantitative as well as qualitative information about the degree of behavioural or psychosocial functioning and are also completed by clinicians. Informant-rating instruments are questionnaires completed by a person who knows the patient well, for instance parents or caregivers. Self-rating instruments are completed by patients themselves. 


\section{Evaluation of psychometric properties and applicability}

The psychometric properties of the instruments were evaluated by D.H. using seven criteria (see Table 6.1) from previous (systematic review) studies. ${ }^{21-24}$ The applicability of the instruments were evaluated based on versions used in previous studies. The following aspects were assessed: availability (an easily obtained instrument), duration of assessment, availability in different languages.

Table 6.1 Criteria to evaluate psychometric properties.

\begin{tabular}{|c|c|c|c|}
\hline & Insufficient & Moderate & Sufficient \\
\hline \multicolumn{4}{|l|}{ 1. Internal consistency ${ }^{a, b, d}$} \\
\hline Cronbach's alpha & $<0.70$ & $0.70-0.80$ & $>0.80$ \\
\hline \multicolumn{4}{|l|}{ 2. Test-retest reliability ${ }^{a, b}$} \\
\hline Spearman / Pearson correlation & $<0.70$ & $0.70-0.80$ & $>0.80$ \\
\hline \multicolumn{4}{|l|}{ 3. Interrater reliability ${ }^{a, b, d}$} \\
\hline $\begin{array}{l}\text { Intra-class correlations (ICC), Pearson } \\
\text { correlation or Kappa between raters }\end{array}$ & $<0.70$ & $0.70-0.80$ & $>0.80$ \\
\hline \multicolumn{4}{|l|}{ 4. Convergent validity ${ }^{a, b}$} \\
\hline Spearman / Pearson correlation & $<0.30$ & $0.30-0.60$ & $>0.60$ \\
\hline \multicolumn{4}{|l|}{ 5. Construct validity ${ }^{\mathrm{a}, \mathrm{b}}$} \\
\hline Principal component analysis & Not confirms structure & & Confirms structure \\
\hline Comparative fit Index (CFI) if available ${ }^{c}$ & & & $>0.95$ \\
\hline Standardized root mean square residual $(\mathrm{SMR})^{\mathrm{c}}$ & & & $<0.9$ \\
\hline \multirow[t]{3}{*}{ 6. Responsiveness ${ }^{a, b, d}$} & Non-significant & & Significant changes: \\
\hline & changes: $p>.05$, or & & $p<.05$, or effect size \\
\hline & effect size $<.40$ & & $>.40$ \\
\hline \multicolumn{4}{|l|}{ 7. Content validity ${ }^{d}$} \\
\hline Aim of instrument is clarified & No & & Yes \\
\hline Focus on dystrophinopathy & No & & Yes \\
\hline Clarification of concepts of subscales & No & & Yes \\
\hline Important items for population & No & & Yes \\
\hline $\begin{array}{l}\text { Interpretability of items (simple, no jargon or } \\
\text { two questions at one time) }\end{array}$ & No & & Yes \\
\hline
\end{tabular}

\section{Results}

The literature search identified 1009 articles based on the inclusion criteria. All articles were accessible. After removing duplicates and excluding articles based on title or abstract, a total of 114 articles remained for full-text screening (see Figure 6.1). Cohen's kappa was calculated to determine the level of agreement between the two independent reviewers (D.H. and J.L.) on title and abstract screening. A moderate agreement was found between the two reviewers, $k=0.72$. After full-text evaluation of the remaining 114 articles, a total of 53 articles were included for final evaluation. 
Reasons for exclusion were (1) conference abstracts, (2) no assessment of behavioural or psychosocial instruments, (3) small sample size <10, (4) study population other than DMD and BMD, or no separate results available for DMD and BMD subpopulation, (5) no cross-sectional or longitudinal study design, and (6) language other than English, Dutch or German. One additional article was extracted from the reference list of the 114 selected articles. This additional article fulfilled the eligibility criteria and was added to the 53 previous included articles, which resulted in a total of 54 included articles (see Figure 6.1).

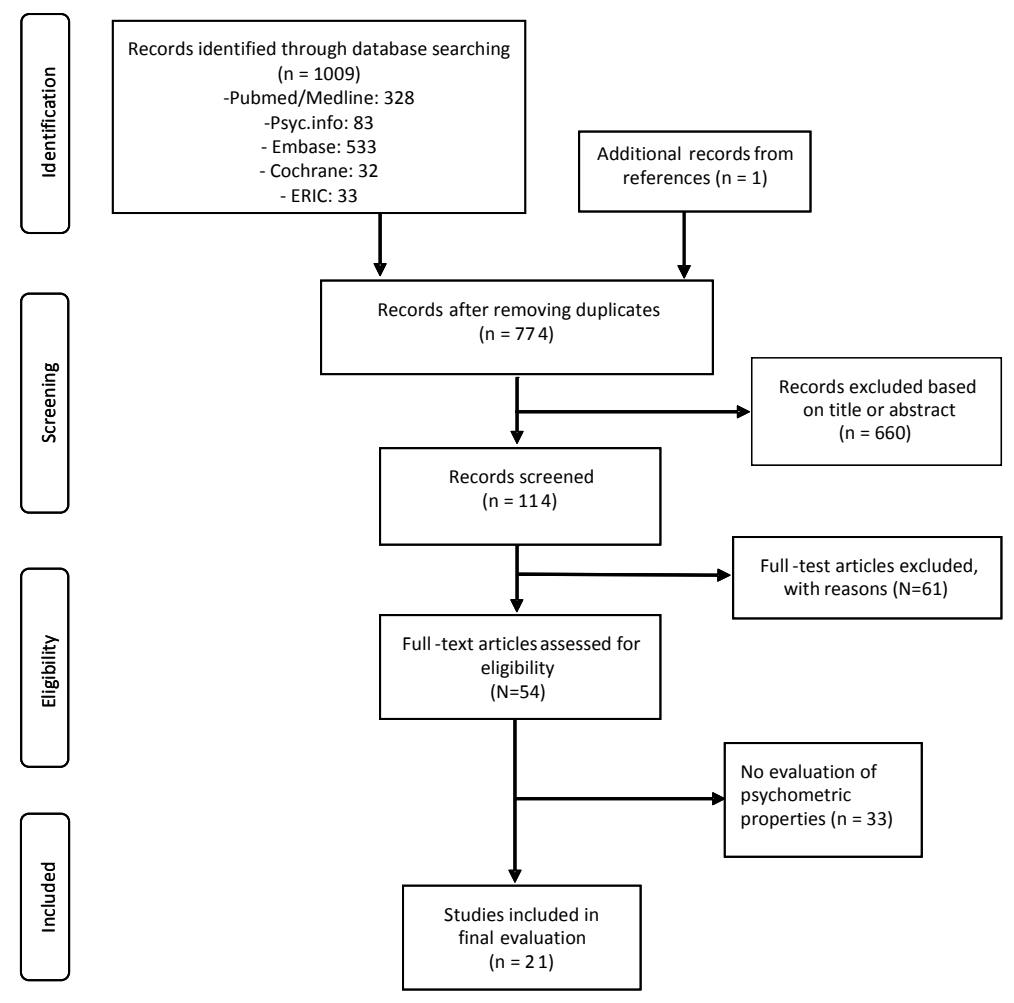

Figure 6.1 Flowchart of the selected articles.

\section{Overview of included studies and instruments}

Among the 54 included articles ( $N=51$ DMD and $N=3 B M D), 61$ different instruments ( $n=1$ clinical observation, $n=12$ semi-structured interviews, $n=22$ informant-ratings, and $n=26$ self-ratings) were used to assess behavioural and psychosocial functioning (see Table 6.2). 


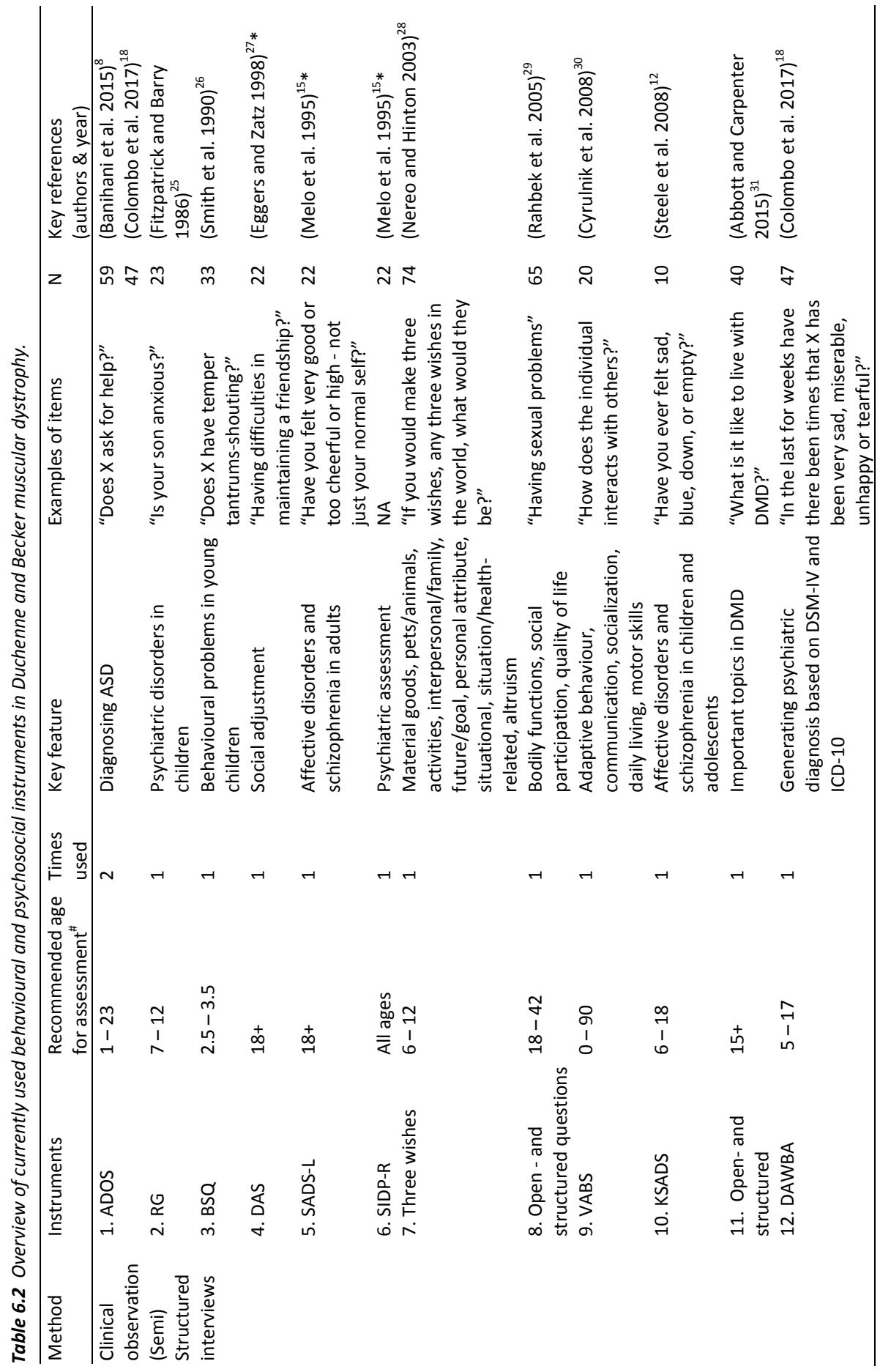




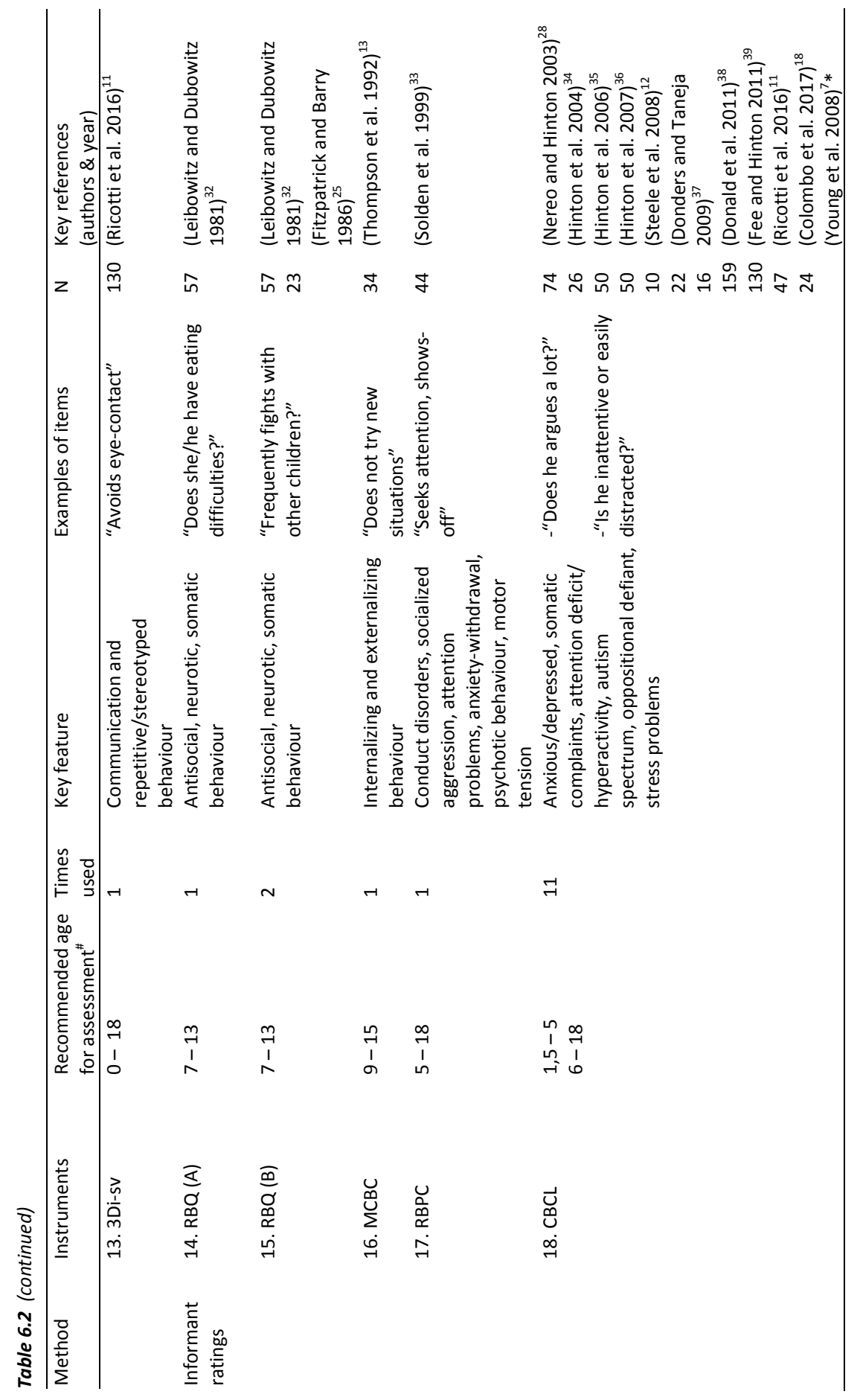




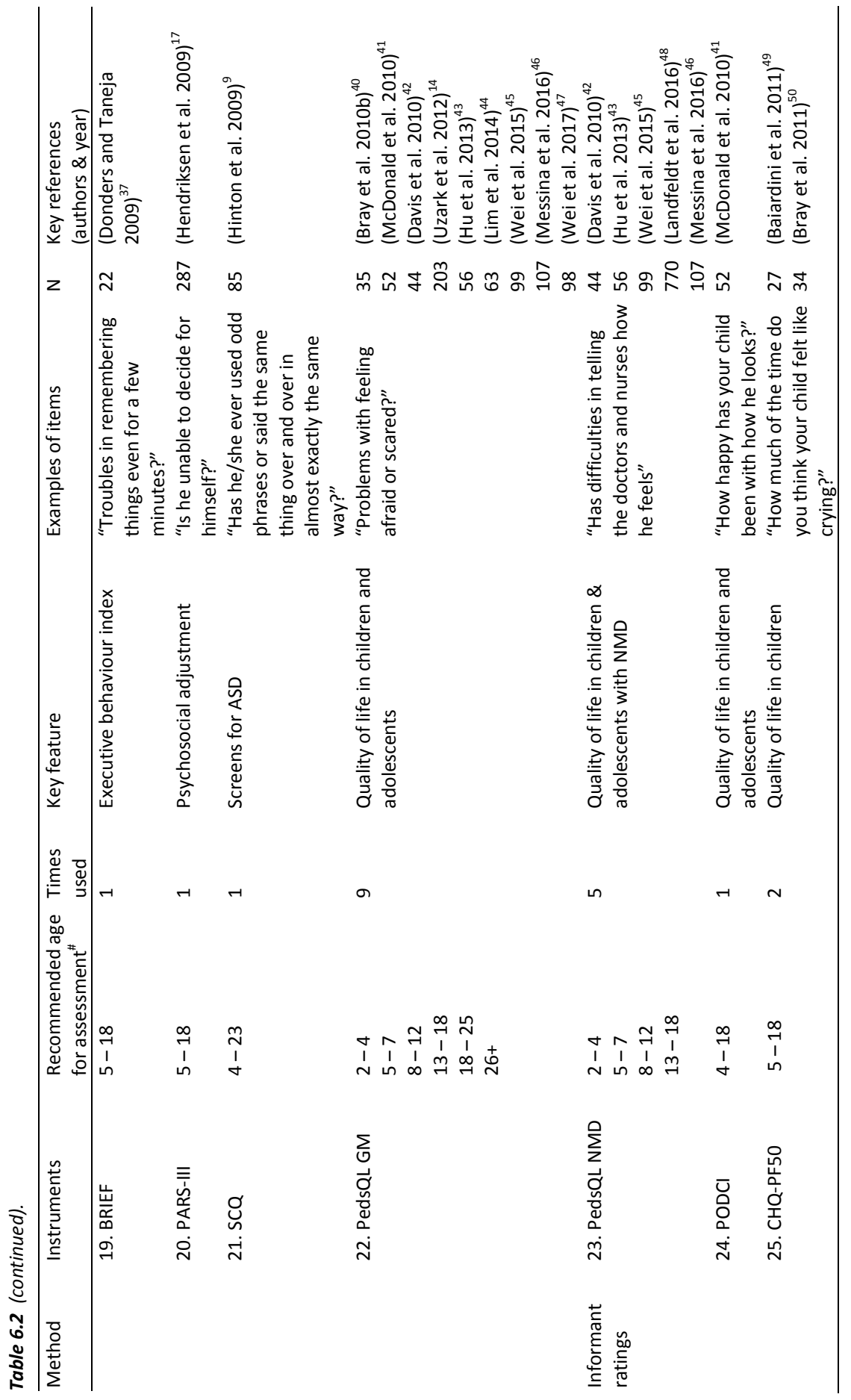




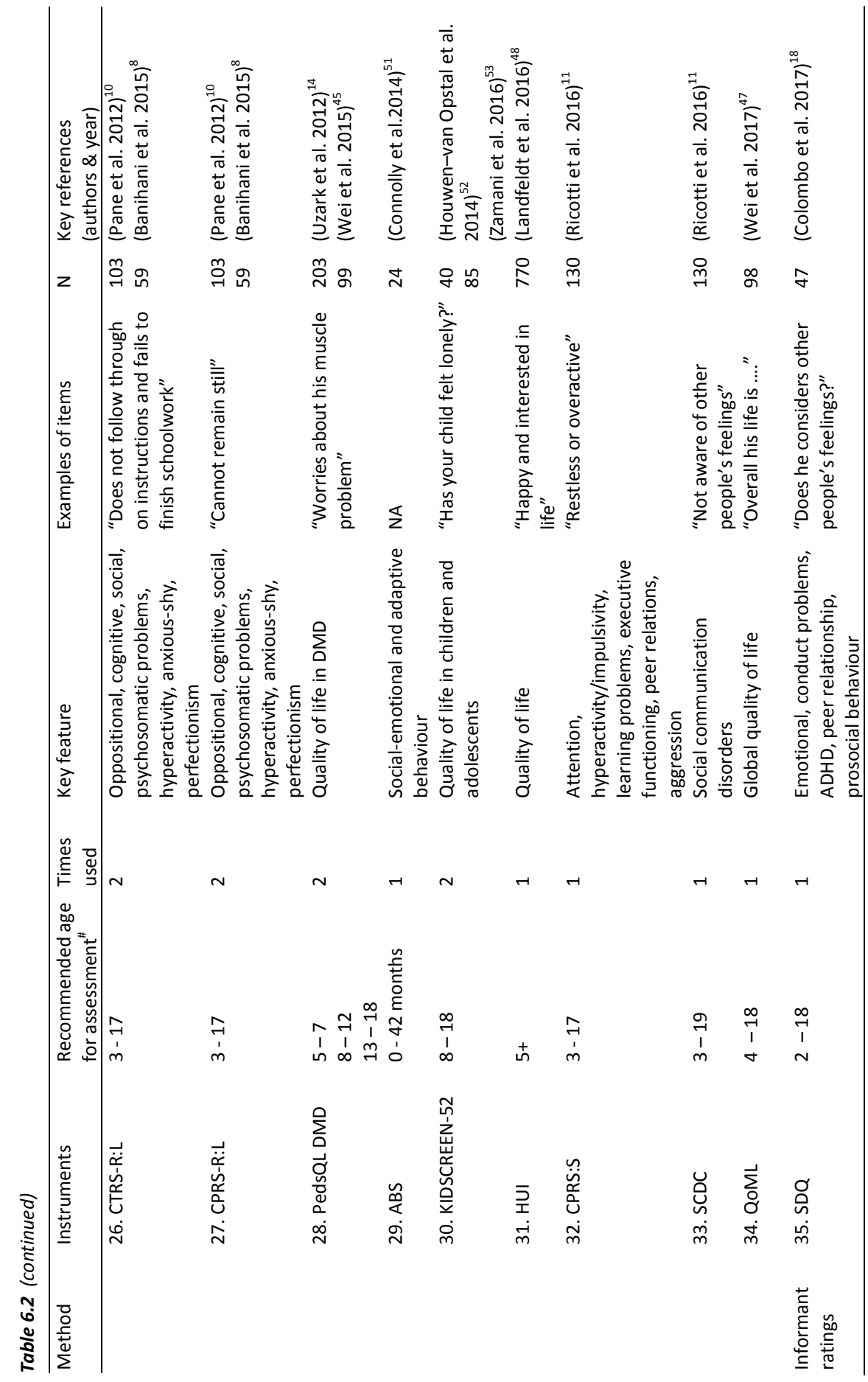




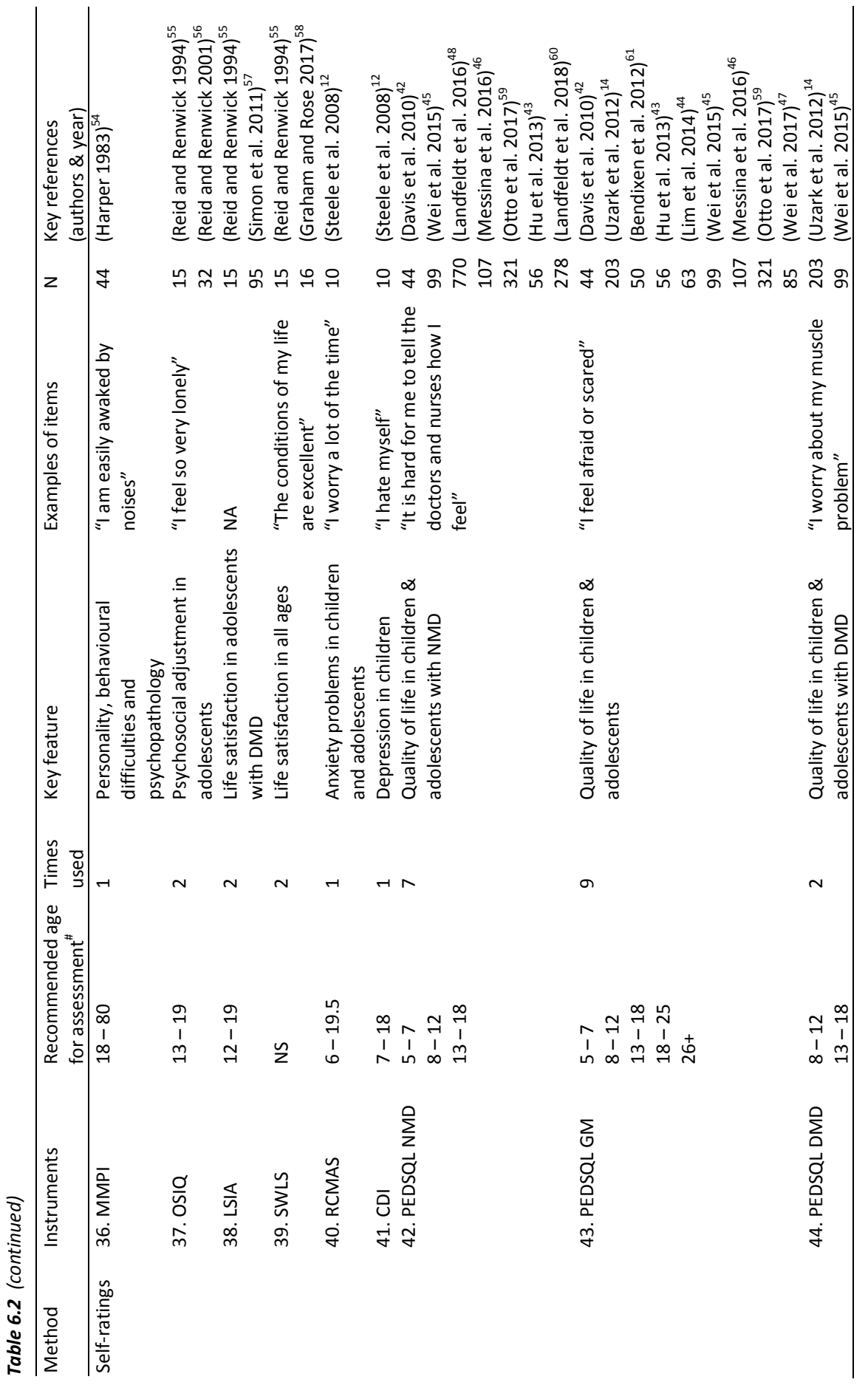




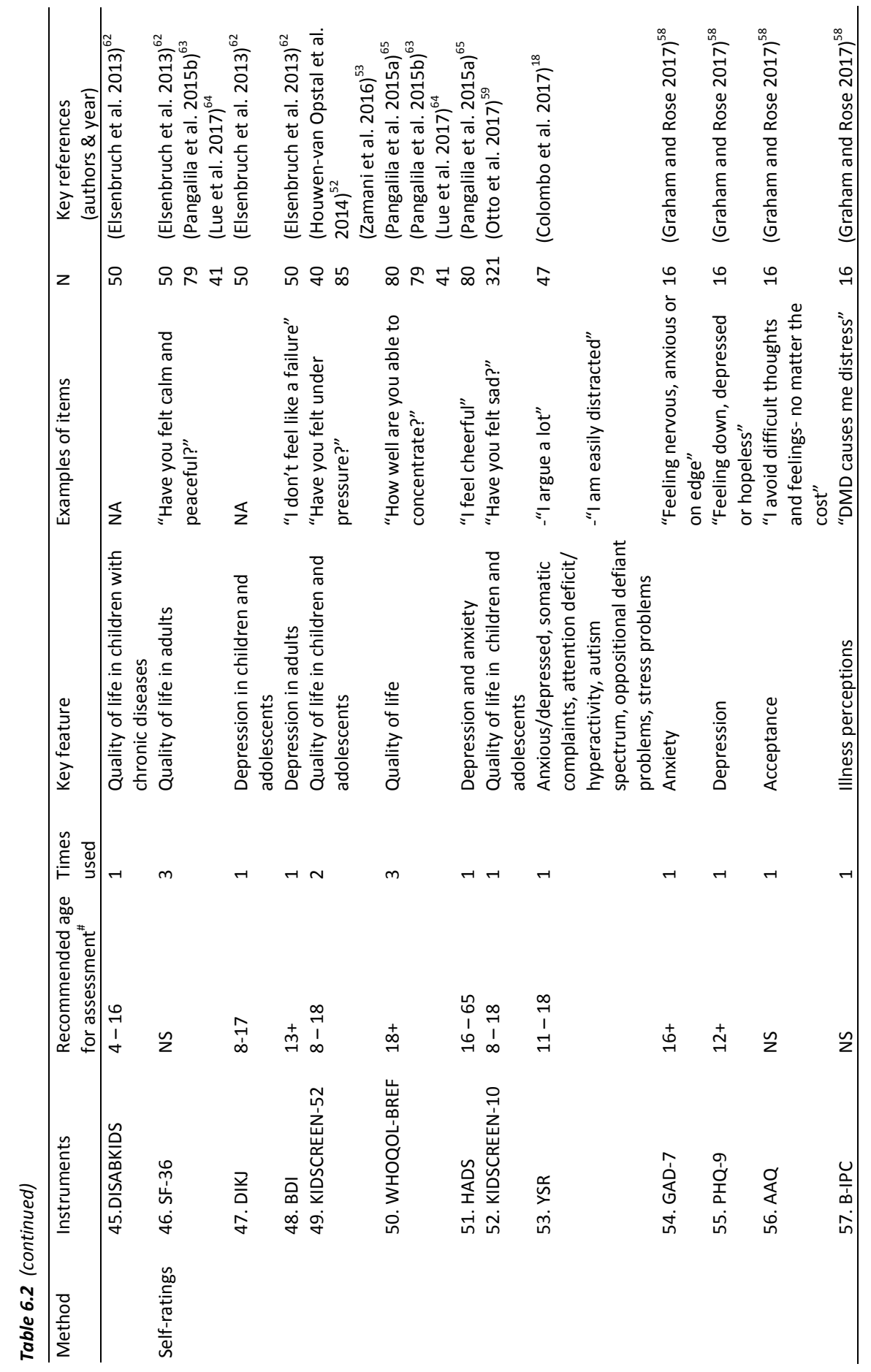




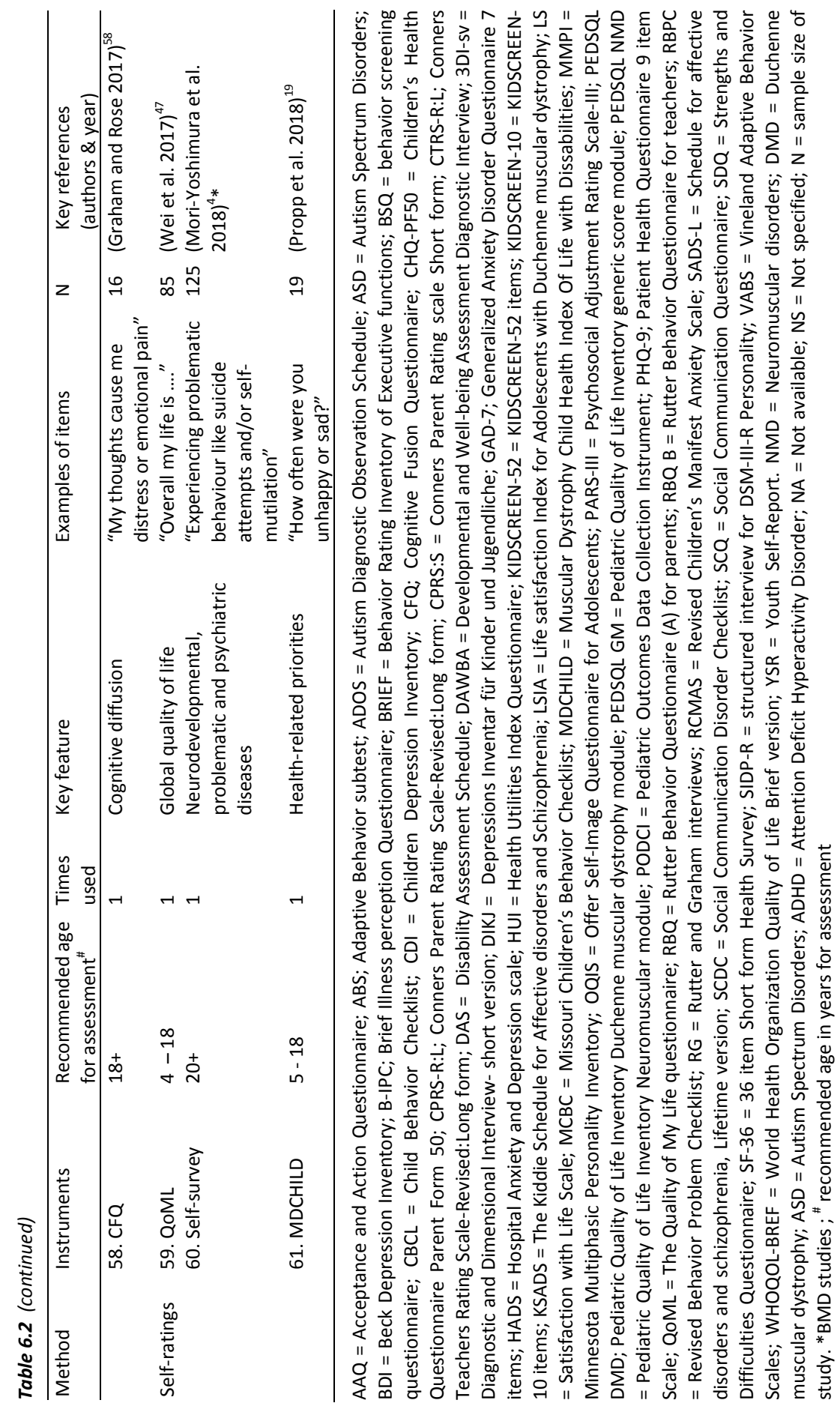


The psychometric properties of these instruments were not evaluated in 33 ( $N=30$ DMD and $\mathrm{N}=3 \mathrm{BMD}$ ) of the 54 articles and were excluded for final evaluation of psychometric properties (see Table 6.3 for excluded instruments). Due to the final selection, a total of 21 articles (all DMD) with 16 instruments ( $n=5$ informant-ratings, $n=6$ self-ratings, and $\mathrm{n}=5$ instruments with informant-ratings and self-ratings) were reviewed. Patients $(\mathrm{N}=2580)$ included in these $\mathrm{N}=21$ studies were aged between 0.7 months and 44 years.

\section{Psychometric properties of included instruments}

Table 6.4 summarizes the psychometric properties of the 16 reviewed instruments. Values of Table 6.4 are psychometric results of the total scale. Subscale information of the instruments is not included. The different forms (i.e. informant-rating and selfrating) of the KIDSCREEN, the Pediatric Quality of Life inventory general module (PedsQL GM), the PedsQL neuromuscular module (PedsQL NMM), and the PedsQL Duchenne module (PedsQL DMD) were assessed separately. As can been seen in Table 6.4, most psychometric information was available from the PARS-III, the PedsQL GM, and the PedsQL NMM.

The PARS-III had good internal consistency (Cronbach's $\alpha=0.91$ ), convergent validity ( $r=-$ 0.65 ), and construct validity (factor analysis confirmed structure of instrument; CFI: 0.96, SRMR: 0.06). No information on inter-rater reliability, test-retest reliability, and responsiveness was available.

The PedsQL GM informant-rating form had good internal consistency (Cronbach's $\alpha=0.83$ ) and the internal consistency of the PedsQL GM self-rating form was moderate to good (Cronbach's $\alpha$ ranged between $0.73-0.87$ ). The inter-rater reliability of both forms was insufficient (informant-rating and self-rating ICC ranged between 0.20-0.64, and $r=0.54)$. Convergent validity was good for the informant-rating form ( $r=0.72)$ but unknown for the self-rating form. Responsiveness was good for both forms (informantrating: $p<.001$, self-rating: $p=.04$ ). No information was available on test-retest reliability and construct validity of the informant-rating form and self-rating form. 
Table 6.3 Excluded instruments for final evaluation

\begin{tabular}{lcccc}
\hline Instruments & $\begin{array}{c}\text { Clinical } \\
\text { observation }\end{array}$ & $\begin{array}{c}\text { (Semi) } \\
\text { Structured } \\
\text { interviews }\end{array}$ & $\begin{array}{c}\text { Informant- } \\
\text { ratings }\end{array}$ & Self-ratings
\end{tabular}

1. ADOS

2. RG

3. BSQ

4. DAS

5. SADS-L

6. SIDP-R

7. Three wishes

8. Open- and structured questions

On bodily functions, social

participation and QOL

9. VABS

\section{KSADS}

11. Open-and structured questions on important topics for DMD

12. DAWBA

13. 3Di-sv

14. $R B Q(A)$

15. $R B Q(B)$

16. $\mathrm{MCBC}$

17. RBPC

18. BRIEF

19. SCQ

20. CTRS-R:L

21. HUI

22. CPRS:S

23. CPRS:L

24. SCDC

25. QoML

26. MMPI

27. SWLS

28. RCMAS

29. CDI

30. DISABKIDS

31. DIKJ

32. BDI

33. HADS

34. AAQ

35. B-IPC

36. CFQ

37. Self-survey

38. MDCHILD

x

X

$\mathrm{x}$

$x$

$\mathrm{x}$

$\mathrm{X}$

$\mathrm{x}$

$\mathrm{X}$

$x$

$\mathrm{x}$

$\mathrm{x}$

$\mathrm{x}$

$\mathrm{X}$

$\mathrm{AAQ}=$ Acceptance and Action Questionnaire; $\mathrm{ADOS}=$ Autism Diagnostic Observation Schedule; $\mathrm{BDI}=\mathrm{Beck}$ Depression Inventory; B-IPC; Brief Illness perception Questionnaire; BRIEF = Behavior Rating Inventory of Executive functions; $\mathrm{BSQ}=$ behavior screening questionnaire; $\mathrm{CDI}=$ Children Depression Inventory; $\mathrm{CFQ}$; Cognitive Fusion Questionnaire; CPRS-R:L; Conners Parent Rating Scale-Revised:Long form; CPRS:S = Conners Parent Rating scale Short form; CTRS-R:L; Conners Teachers Rating Scale-Revised:Long form; DAS = Disability Assessment Schedule; DAWBA = Developmental and Well-being Assessment Diagnostic Interview; 3DI-sv = 
Diagnostic and Dimensional Interview- short version; DIKJ = Depressions Inventar für Kinder und Jugendliche; HADS = Hospital Anxiety and Depression scale; HUI = Health Utilities Index Questionnaire; KSADS = The Kiddie Schedule for Affective disorders and Schizophrenia; MCBC = Missouri Children's Behavior Checklist; MDCHILD = Muscular Dystrophy Child Health Index Of Life with Dissabilities; MMPI = Minnesota Multiphasic Personality Inventory; $\mathrm{QoML}=$ The Quality of My Life questionnaire; $\mathrm{RBQ}=$ Rutter Behavior Questionnaire (A) for parents ; $\mathrm{RBQ} B=$ Rutter Behavior Questionnaire for teachers; RBPC = Revised Behavior Problem Checklist; RG = Rutter and Graham interviews; RCMAS = Revised Children's Manifest Anxiety Scale; SADS-L = Schedule for affective disorders and schizophrenia, Lifetime version; SCDC = Social Communication Disorder Checklist; SCQ = Social Communication Questionnaire; SIDP-R = structured interview for DSM-III-R Personality; VABS = Vineland Adaptive Behavior Scales.

Table 6.4 Psychometric properties of included instruments

\begin{tabular}{|c|c|c|c|c|c|c|c|}
\hline Method & Instrument & $\begin{array}{c}\text { Internal } \\
\text { consistency }\end{array}$ & $\begin{array}{c}\text { Test- retest } \\
\text { reliability }\end{array}$ & $\begin{array}{l}\text { Inter-rater } \\
\text { reliability }\end{array}$ & $\begin{array}{c}\text { Convergent } \\
\text { validity }\end{array}$ & $\begin{array}{c}\text { Construct } \\
\text { validity }\end{array}$ & Responsiveness \\
\hline Informant- & 1. PARS-III & + & $?$ & $?$ & + & + & $?$ \\
\hline \multirow[t]{4}{*}{ ratings } & 2. $\mathrm{CBCL}$ & ? & $?$ & $\pm /-$ & $+/ \pm$ & $?$ & $?$ \\
\hline & 3. $\mathrm{PODCl}$ & ? & $?$ & ? & + & $?$ & $?$ \\
\hline & 4. CHQ-PF50 & \pm & $?$ & $?$ & $?$ & $?$ & $?$ \\
\hline & 5. $A B S$ & $?$ & $?$ & $?$ & $?$ & $?$ & - \\
\hline Self- & 6. OSIQ & + & $?$ & $?$ & $?$ & $?$ & $?$ \\
\hline \multirow[t]{5}{*}{ ratings } & 7. LSIA & + & $?$ & $?$ & + & $?$ & $?$ \\
\hline & 8. YSR & ? & $?$ & $\pm /-$ & $?$ & $?$ & $?$ \\
\hline & 9. SDQ & ? & $?$ & $?$ & $+/ \pm$ & $?$ & $?$ \\
\hline & 10. SF-36 & $?$ & $?$ & $?$ & $\pm /-$ & $?$ & $?$ \\
\hline & 11. WHOQOL-BREF & ? & $?$ & $?$ & $\pm /-$ & $?$ & $?$ \\
\hline Informant & 12. PEDSQL GM & & & & & & \\
\hline$\&$ self- & Informant form & + & $?$ & - & + & $?$ & + \\
\hline \multirow[t]{12}{*}{ ratings } & $\begin{array}{l}\text { Self-rating form } \\
\text { 13. PEDSQL NMD }\end{array}$ & $+/ \pm$ & $?$ & - & na & $?$ & + \\
\hline & Informant form & + & $+/ \pm$ & $\pm /-$ & na & ? & + \\
\hline & Self-rating form & + & $\pm /-$ & $\pm /-$ & na & - & - \\
\hline & 14. PEDSQL DMD & & & & & & \\
\hline & Informant form & $+/ \pm$ & $?$ & - & na & $?$ & $?$ \\
\hline & Self-rating form & \pm & $?$ & - & na & $?$ & $?$ \\
\hline & 15. KIDSCREEN-52 & & & & & & \\
\hline & Informant form & ? & $?$ & $\pm /-$ & $?$ & $?$ & $?$ \\
\hline & Self-rating form & $?$ & $?$ & $\pm /-$ & $?$ & $?$ & $?$ \\
\hline & 16. KIDSCREEN-10 & & & & & & \\
\hline & Informant form & \pm & $?$ & $?$ & $?$ & $?$ & $?$ \\
\hline & Self-rating form & \pm & $?$ & $?$ & $?$ & $?$ & $?$ \\
\hline
\end{tabular}

$\overline{\mathrm{ABS}}=$ Adaptive Behavior subtest of Bayley; $\mathrm{CBCL}=$ Child Behavior Checklist; CHQ-PF50 = Children's Health Questionnaire Parent Form 50; KIDSCREEN-52 = KIDSCREEN 52 items; KIDSCREEN-10 = KIDSCREEN 10 items; $\mathrm{LSIA}=$ Life satisfaction Index for Adolescents with Duchenne muscular dystrophy; OSIQ = Offer Self-Image Questionnaire for Adolescents; PARS-III = Psychosocial Adjustment Rating Scale-III; PEDSQL DMD; Pediatric Quality of Life Inventory Duchenne muscular dystrophy module; PEDSQL GM = Pediatric Quality of Life Inventory generic score module; PEDSQL NMD = Pediatric Quality of Life Inventory Neuromuscular module; $\mathrm{PODCl}=$ Pediatric Outcomes Data Collection Instrument; SDQ = Strengths and Difficulties Questionnaire; SF$36=36$ item Short form Health Survey; WHOQOL-BREF = World Health Organization Quality of Life Brief version; YSR = Youth Self-Report. +: sufficient ; \pm : moderate; -: insufficient; ?: unknown/unclear; na: not applicable 
The PedsQL NMM informant-rating form and self-rating form had good internal consistency (Cronbach's $\alpha$ informant-rating form: $0.86-0.87$ and self-rating form: 0.81-0.92). Test-retest reliability of the informant-rating form was sufficient $(r=0.85-0.88)$ and moderate to insufficient $(r=0.66-0.75)$ for the self-rating form. The inter-rater reliability of both forms ranged from moderate to insufficient (ICC ranged between 0.51-0.78). Convergent validity was good for the informant-rating form $(r=0.60-0.71)$ and the self-rating form ( $r=0.65-0.67)$. No information was available on construct validity of the informant-rating form. Construct validity was insufficient for the self-rating form, since the structure of the form was not confirmed by principal component analysis. Responsiveness was good for the informant-rating form $(p=.005)$ but insufficient for the self-rating form $(p=.77)$.

\section{Content validity and applicability of included instruments}

Results of content validity and applicability of the 16 reviewed instruments are displayed in Table 6.5. All instruments met criterion 1, signifying that instruments had specific measurement aims. Only two instruments were specifically developed for dystrophinopathy and met criterion 2: the Life Satisfaction Index for Adolescent with Duchenne muscular dystrophy (LSIA) and the PedsQL DMD. All instruments consisted of specified concepts, measured by separate subscales and items (criterion 3), except for the PedsQL NMM self-rating form. Subscales of the PedsQL NMM self-rating form contain non-distinctive items, for instance, "it is hard for me to tell the doctors and nurses how I feel" and "it is hard for me to ask doctors and nurses questions." All instruments met criterion 4 and measured a degree of behavioral or psychosocial functioning relevant for the dystrophinopathy population, though items of two instruments (i.e. Child Behavior Checklist; CBLC Offer Self-Image Questionnaire; OSIQ) are less applicable in dystrophinopathy due to the progressive physical immobility. Less applicable items are for example can't sit still, being restless' (CBCL-ADHD subscale), 'runs away from home' (CBCL-rule breaking behavior subscale), or 'I love the recent changes in my body' (OSIQ). Several instruments did not fulfill criterion 5 , since the items contain more than one question at the same time. In addition, items of the PedsQL NMM self-rating form for toddlers and young children (i.e. "hard to gain or lose weight'; do not have the equipment one needs) are not applicable or too difficult to understand. With regard to the applicability of the instruments, it was found that all instruments were available in English. Most instruments $(n=9)$ were freely available by website, four by author, and three by publisher. Administration time of nine instruments was $<10$ minutes and of seven instruments the time varied from 10-50 minutes. 


\section{Discussion}

The purpose of this systematic review was to provide an overview of instruments used to assess behavioural and psychosocial functioning of patients with DMD and BMD, as well as to review the psychometric properties and applicability of these instruments. A total of 61 different instruments have been used in 51 DMD studies and 3 BMD studies. Our review showed that most previous studies provide limited psychometric information. The majority of instruments being used are informant-rating and selfrating instruments. The most common used ( $\mathrm{N}=11$ studies) is the $\mathrm{CBCL}^{66}$ but only two studies reported psychometric information on convergent validity (ranging from poor to moderate) and inter-rater reliability (ranging from moderate to good. ${ }^{18,39}$ Additionally, the content validity of $\mathrm{CBCL}$ is less sufficient for patients with motor impairments when questions involve normal motor abilities. This can result in underidentification of behavioural comorbidities due to lower scores in for instance hyperactive or oppositional, aggressive behaviour. ${ }^{67}$ Furthermore, the $\mathrm{CBCL}$ is developed for screening of various behavioural and emotional symptoms and is not intended to be a diagnostic instrument for establishing definite DSM-5 diagnoses, ${ }^{68}$ such as ADHD. ${ }^{69}$ To accurately diagnose ADHD clinicians should be able to differentiate symptoms of inattention, hyperactivity, and impulsivity. The symptom items of the CBCL-ADHD subscale have no conceptual link with the diagnostic criteria for ADHD. ${ }^{69}$ Other instruments, for instance, the Conners Parent Rating Scale-Revised (CPRS-R) or the SDQ differentiate and ascertain ADHD symptoms more accurately. Since the items of the CPRS-R and SDQ subscales are selected on the basis of nosologically concepts (i.e. DSM criteria and International Statistical Classification of diseases and related Health Problems: ICD-10) as well as on factor analysis. ${ }^{69-71}$ This likely explains that Colombo et al. ${ }^{18}$ recently found an ADHD comorbidity of $0 \%$ in their DMD sample based on $\mathrm{CBCL}$ screening, while Banihani et al. ${ }^{8}$ found a prevalence rate as high as $32 \%$ for ADHD using CPRS-R for ADHD. These data confirm that the prevalence rates of behavioural comorbidities in DMD as previously reported largely depend on the instruments that were used and should, therefore, be considered with caution. The majority of previous studies used instruments with unevaluated or poor psychometric properties, or instruments that are not developed to differentiate behavioural symptoms.

\section{Psychometric properties of included instruments}

For the current systematic review disease specific psychometric information was available for 21 DMD studies with 16 instruments being reviewed, including both 
informant-ratings and self-ratings. The 33 excluded studies used significantly different instruments including clinical observations and semi-structured interviews, in addition to informant-rating and self-rating instruments. Thereby, this review only describes psychometric properties of informant-rating and self-rating instruments for DMD. With regard to the psychometric properties of the included instruments, more in depth evaluation was found for the PARS-III, the PedsQL GM, and the PedsQL NMM. The PARS-III proves to be an adequate instrument and is therefore recommended in the standards of care for DMD. ${ }^{16}$ However, additional validation by replication of reported psychometric results is recommended as well as data on inter-rater reliability, test retest reliability, and responsiveness. The PedsQL GM informant-rating and self-rating form have relatively good properties, both having only poor inter-rater reliability. Data on PedsQL NMM informant-rating form varies in previous studies and it inter-rater reliability is low. Moderate to poor psychometric results are noted for the PedsQL NMM self-rating form, suggesting that it is no valid instrument for DMD. The inter-rater reliability of both PedsQL modules (GM and NMM) are low, reflecting a discrepancy between patients and their parents/caregivers. This is in line with previous studies showing that parents/caregivers overestimate the magnitude of problems due to their own concerns and perceptions of the illness. ${ }^{33,40}$ This could explain the notable difference between behavioural and psychosocial problems reported by patients and caregivers. $^{42,52,44}$ Additional validation on psychometric properties of both PedsQL modules and their separate forms is necessary, before using them for clinical or research trials.

With respect to the three (SDQ, GAD-7, and $\mathrm{PHQ}-9$ ) remaining recommended instruments of the recently published standards of care for $\mathrm{DMD},{ }^{16}$ two instruments (GAD-7 and PHQ-9) have no disease specific psychometric information. However, previous studies reported that the GAD-7 is a valid instrument for screening of generalized anxiety disorders as well as for panic, social anxiety, and post-traumatic stress disorders. ${ }^{72,73}$ Nevertheless, the GAD-7 provides only a probable diagnosis and further evaluation is necessary to diagnose anxiety. Furthermore, the psychometric properties of the PHQ-9 have been evaluated by multiple clinical studies. This instrument seems favourable in screening for depression in primary and mental health care and is equal or superior to other depression measures. ${ }^{74}$ However, previous studies on the GAD-7 and the PHQ-9 evaluated the reliability and validity of these instruments in adult patients and the usefulness for DMD should be assessed accordingly. The third instrument, the SDQ, poses limited psychometric information and Colombo et al. ${ }^{18}$ showed that the SDQ has a moderate to good validity in $\mathrm{N}=47$ males with DMD. However, the SDQ has been extensively evaluated in children and 
adolescents with or without a chronic illness and it is believed that it is a reliable and valid screener for psychiatric. ${ }^{75-80}$ In addition, a higher specificity of the SDQ informantrating $(0.85)$ has been noted compared to the $\mathrm{CBCL}(0.72)$, meaning that the SDQ will result in less overestimation of true behavioural comorbidity. Overall, the SDQ should be considered as a valid and robust instrument for future research and clinical settings, though care should be taken when using the SDQ predictive algorithm to screen for ADHD comorbidity. ${ }^{71}$ Further evaluation of the SDQ psychometric properties for the dystrophinopathy population is recommended.

\section{Content validity and applicability of included instruments}

The current review systematically evaluated content validity and applicability of the 16 included instruments. Only the LSIA and PedsQL DMD fulfilled the five content validity criteria and may thus be suitable for DMD care. However, our psychometric results revealed sufficient and reliable results for the LSIA, but insufficient results for the PedsQL DMD. Additional research on the reliability of both instruments is recommended. Furthermore, of the 16 included instruments, the majority had items that were (1) limited or not applicable to the dystrophinopathy population, (2) or too difficult to answer for patients or parents/caregivers. In addition, the majority of the included instruments were relatively easily accessible by website and could be filled in quickly (<10 $\mathrm{min})$.

\section{Limitations}

This review was designed to capture all behavioural and psychosocial instruments currently used in cross-sectional and longitudinal studies. As a consequence, potentially good instruments used in studies with other designs (e.g. case-report or conference abstracts) have been excluded. For instance, the MDCHILD could not be included in the current review, since no information was available on psychometric properties. ${ }^{19}$ Furthermore, we excluded studies with possible adequate instruments when no separate information was available for the dystrophinopathy population. Excluded studies, for instance, used the Autism Diagnostic Interview-Revised, ${ }^{9}$ Experience Sampling Method, ${ }^{81}$ California Psychological Inventory, ${ }^{82}$ Personal Assessment Inventory-Depression, ${ }^{82}$ Child Health Questionnaire-Child form- $87,{ }^{83}$ Multidimensional Scale of Perceived Social Support (Wilson et al. 2006), Quality of Life Profile Questionnaire, ${ }^{84,85}$ and Strips of Life with Emoticons Questionnaire ${ }^{86}$ in patients with other neuromuscular disorders (e.g. spinal muscular atrophy, myopathy, and giant axonal neuropathy). An important strength of our study is the use of the psychometric property criteria. However, at the same time this approach limits the finding of 
adequate instruments. Previous studies, for instance, used less stringent criteria to assess inter-rater reliability between the informant-rating and self-rating form of the PedsQL GM and PedsQL NMM. ${ }^{14,42}$ Despite the less stringent criteria, the outcomes of Davis and colleagues ${ }^{42}$ and Uzark and colleagues ${ }^{14}$ were in line with the findings of the current review concerning the poor inter-reliability between the informant-rating and self-rating form of both PedsQL modules. Furthermore, for final evaluation, we excluded studies that reported no information on psychometric properties, content validity, and applicability for the dystrophinopathy population. Therefore, all BMD studies were excluded and generalizations of our results regarding the entire dystrophinopathy population should be made with caution. Additionally, the MDCHILD could not be included in the current review, since no information was available on psychometric properties for the dystrophinopathy population. ${ }^{19}$

\section{Clinical recommendations}

The current review provides no gold standard for methods of assessment, but based on the findings the PARS-III, LSIA, and PedsQL GM can be valid instruments for screening of psychosocial problems in DMD. Nonetheless, when using the PedsQL GM we recommend to administer both the informant-rating and self-rating form, to limit the error variance caused by disagreements between patients and parents/caregivers perspectives.

Appropriate instruments for screening of behavioural problems in DMD may be the SDQ for paediatric patients, and the GAD-7 and PHQ-9 for adult patients. Additional research on psychometric properties of these instruments for DMD as well for BMD is crucial, and definite outcomes of these screening instruments should be considered with caution.

For definite diagnostics, we recommend to use the gold standard assessment method for behavioural disorders. This is referred to as the multi-method, multi-source, multisetting assessment and implies using different assessment methods (e.g. behavioural observations, structured clinical interviews, informant-ratings, and self-ratings), different sources (e.g. patients, parents/caregivers, teachers, clinicians), and different settings (e.g. at home, school, the clinic) to get a comprehensive representation of the child's or adolescent's behavioural, emotional, and psychosocial functioning. ${ }^{87} \mathrm{We}$ believe that this method is particularly important for the ageing high-risk dystrophinopathy population, whereof $20 \%$ of patients have more than one comorbid behavioural disorder. ${ }^{5,8.9,11-14}$ This multi-method-, source-, setting assessment reduces error variance, resulting in less under-identification of behavioural comorbidities in this 
high-risk population. Each neuromuscular team should include a mental health professional (psychologist or psychiatrist) with experience in neurodevelopmental, behavioural, and medical conditions, who routinely screens a patients' behavioural and psychosocial functioning every 2-3 years and applies this multi-method-,source-, setting assessment when necessary for definite diagnostics. ${ }^{16}$ Adequate and early detection of behavioural comorbidities can greatly facilitate targeted treatment. We know, for instance, that in the general ADHD population, underdiagnosed ADHD problems put patients at higher risk for educational underachievement, behavioural comorbidities like depression, impairments in social relations, and a reduced quality of life, that can extend throughout adulthood. ${ }^{88-90}$ According to our review, only one previous DMD study applied this multi-method-, source-, setting assessment using the DSM-IV criteria together with two screenings instruments, the Conners Parent and Conners Teacher Rating Scales, to diagnose ADHD comorbidity in their DMD sample. ${ }^{8}$ Two additional studies administered this method partly and evaluated behavioural problems using structured interviews such as the DAWBA, ADOS, and 3Di-Sv in addition to multiple informant-rating and self-rating instruments. ${ }^{11,18}$

\section{Conclusion}

The present review describes a wide variety of instruments used to assess behavioural and psychosocial functioning in the dystrophinopathy population. We argue that the interpretation of behavioural disorders and psychosocial problems as reported in previous studies using these instruments should be taken with caution. Our review shows that for psychosocial screening, the PARS-III, PedsQL GM, and LSIA can be valid. For behavioural screening, the SDQ, GAD-7, and PHQ-9, may be appropriate. Other screening instruments that are valid and appropriate for the dystrophinopathy population may be used as well, but their outcomes should be considered with caution. Additional psychometric data on instruments for screening of behavioural and psychosocial functioning of the dystrophinopathy population is necessary. Since the ageing dystrophinopathy population is confronted with long-lasting or new mental health issues that negatively influence their quality of life. A mental health professional should routinely screen a patient's behavioural and psychosocial functioning, preferably every 2-3 years and particularly during transitioning (e.g. childhood to adolescence and adolescence to adulthood). For definite diagnostics, we believe that a that a multimethod-, source-, setting assessment is the most appropriate method of assessment in this unique population. Given the complexity of behavioural comorbidities, clinicians 
and researchers should never diagnose conditions based on the result of one screening instrument.

\section{Acknowledgement}

The authors would like to thank Spieren voor Spieren, Duchenne Parent Project NL for their funding. Special thanks to mw. I. Gijselhart for her help in creating the searches. 


\section{References}

1. Doorenweerd N, Mahfouz A, van Putten M, Kaliyaperumal R, t'Hoen PA, Hendriksen JG, et al. Timing and localization of human dystrophin isoform expression provide insights into the cognitive phenotype of Duchenne muscular dystrophy. Sci Rep 2017;7(1):12575.

2. Hendriksen RGF, Hoogland G, Schipper S, Hendriksen JGM, Vles JSH, Aalbers MW. A possible role of dystrophin in neuronal excitability: a review of the current literature. Neurosci Biobehav Rev 2015;51:255-262.

3. Muntoni F, Torelli S, Ferlini A. Dystrophin and mutations: one gene, several proteins, multiple phenotypes. Lancet Neurol 2003;2(12):731-740.

4. Mori-Yoshimura M, Mizuno Y, Yoshida S, Minami N, Yonemoto N, Takeuchi F, et al. (2018). Social involvement issues in patients with Becker muscular dystrophy: A questionnaire survey of subjects from a patient registry. Brain Dev 2018;40(4):268-277.

5. Hendriksen JGM, Vles JSH. Neuropsychiatric disorders in males with duchenne muscular dystrophy: frequency rate of attention-deficit hyperactivity disorder (ADHD), autism spectrum disorder, and obsessive--compulsive disorder. J Child Neurol 2008;23(5):477-481.

6. Snow WM, Anderson JE, Jakobson LS. Neuropsychological and neurobehavioral functioning in Duchenne muscular dystrophy: a review. Neurosci Biobehav Rev 2013;37(5):743-752.

7. Young HK, Barton BA, Waisbren S, Dale LP, Ryan MM, Webster RI, et al. Cognitive and psychological profile of males with becker muscular dystrophy. J Child Neurol 2008;23(2):155-162.

8. Banihani R, Smile S, Yoon G, Dupuis A, Mosleh M, Snider A, et al. (2015). Cognitive and Neurobehavioral Profile in Boys With Duchenne Muscular Dystrophy. J Child Neurol 2015;30(11):1472-1482.

9. Hinton VJ, Cyrulnik SE, Fee RJ, Batchelder A, Kiefel JM, Goldstein EM, et al. Association of Autistic Spectrum Disorders With Dystrophinopathies. Pediatr Neurol 2009;41(5):339-346.

10. Pane M, Lombardo ME, Alfieri P, D'Amico A, Bianco F, Vasco G, et al. (2012). Attention deficit hyperactivity disorder and cognitive function in Duchenne muscular dystrophy: phenotype-genotype correlation. J Pediatr 2012;161(4):705-709. e701.

11. Ricotti V, Mandy WP, Scoto M, Pane M, Deconinck N, Messina S, et al. Neurodevelopmental, emotional, and behavioural problems in Duchenne muscular dystrophy in relation to underlying dystrophin gene mutations. Dev Med Child Neurol 2016;58(1):77-84.

12. Steele M, Taylor E, Young C, McGrath P, Lyttle BD, Davidson B. Mental health of children and adolescents with Duchenne muscular dystrophy. Dev Med Child Neurol 2008;50(8):638-639.

13. Thompson RJ, Zeman JL, Fanurik D, Sirotkin-Roses M. The role of parent stress and coping and family functioning in parent and child adjustment to Duchenne muscular dystrophy. J Clin Psychol 1992;48(1):11-19.

14. Uzark K, King E, Cripe L, Spicer R, Sage J, Kinnett K, et al. Health-related quality of life in children and adolescents with Duchenne muscular dystrophy. Pediatrics 2012;130(6):e1559-e1566.

15. Melo M, Lauriano V, Gentil V, Eggers S, Del Bianco SS, Gimenez PR, et al. (1995). Becker and limb-girdle muscular dystrophies: A psychiatric and intellectual level comparative study. Am J Med Genet 1995;60(1):33-38.

16. Birnkrant DJ, Bushby K, Bann CM, Apkon SD, Blackwell A, Colvin MK, et al. Diagnosis and management of Duchenne muscular dystrophy, part 3: primary care, emergency management, psychosocial care, and transitions of care across the lifespan. Lancet Neurology 2018;17(5):445-455.

17. Hendriksen JGM, Poysky JT, Schrans DG, Schouten EG, Aldenkamp AP, Vles JSH. Psychosocial adjustment in males with Duchenne muscular dystrophy: psychometric properties and clinical utility of a parent-report questionnaire. J Pediatr Psychol 2009;34(1):69-78.

18. Colombo P, Nobile M, Tesei A, Civati F, Gandossini S, Mani E, et al. Assessing mental health in boys with Duchenne muscular dystrophy: Emotional, behavioural and neurodevelopmental profile in an Italian clinical sample. Eur J Paediatr Neurol 2017;21(4):639-647.

19. Propp R, McAdam L, Davis AM, Salbach NM, Weir S, Encisa C, et al. (2018). Development and content validation of the Muscular Dystrophy Child Health Index of Life with Disabilities questionnaire for children with Duchenne muscular dystrophy. Dev Med Child Neurol 2019;61(1):85-81.. 
20. Liberati A, Altman DG, Tetzlaff J, Mulrow C, Gøtzsche PC, loannidis JP, et al. The PRISMA statement for reporting systematic reviews and meta-analyses of studies that evaluate health care interventions: explanation and elaboration. PLoS Med 2009;6(7):e1000100.

21. Hu LT, Bentler PM. Cutoff criteria for fit indexes in covariance structure analysis: Conventional criteria versus new alternatives. Struct Equa Modeling 1999;6(1):1-55.

22. Smeets SM, Ponds RW, Verhey FR, van Heugten CM. Psychometric properties and feasibility of instruments used to assess awareness of deficits after acquired brain injury: a systematic review. J Head Trauma Rehabil 2012;27(6):433-442.

23. Terwee CB, Bot SD, de Boer MR, van der Windt DA, Knol DL, Dekker J, et al. Quality criteria were proposed for measurement properties of health status questionnaires. J Clin Epidemiol 2007;60(1):3442.

24. Visser-Meily JA, Post MW, Riphagen II, Lindeman E. Measures used to assess burden among caregivers of stroke patients: a review. Clin Rehabil 2004;18(6):601-623.

25. Fitzpatrick C, Barry C. (1986). Communication within families about Duchenne muscular dystrophy. Dev Med Child Neurol 1986;28(5):596-599.

26. Smith RA, Sibert JR, Harper PS. (1990). Early development of boys with Duchenne muscular dystrophy. Dev Med Child Neurol 1990;32(6):519-527.

27. Eggers S, Zatz M. (1998). Social adjustment in adult males affected with progressive muscular dystrophy. Am J Med Genet 1998;81(1):4-12.

28. Nereo NE, Hinton VJ. Three Wishes and psychological functioning in boys with Duchenne muscular dystrophy. J Dev Behav Pediatr 2003;24(2):96-103.

29. Rahbek J, Werge B, Madsen A, Marquardt J, Steffensen BF, Jeppesen J. Adult life with Duchenne muscular dystrophy: observations among an emerging and unforeseen patient population. Pediatr Rehabil 2005;8(1):17-28.

30. Cyrulnik SE, Fee RJ, Batchelder A, Kiefel J, Goldstein E, Hinton VJ. Cognitive and adaptive deficits in young children with Duchenne muscular dystrophy (DMD). J Int Neuropsychol Soc 2008;14(5):853-861.

31. Abbott D, Carpenter J. "The Things That Are inside of You Are Horrible": Children and Young Men with Duchenne Muscular Dystrophy Talk about the Impact of Living with a Long-Term Condition. Child Care Pract 2015;21(1):67-77.

32. Leibowitz D, Dubowitz V. Intellect and behaviour in Duchenne muscular dystrophy. Dev Med Child Neurol 1981;23(5):577-590.

33. Solden J, Guildea ZES, Antao V, Street E, Sibert JR. Behavioural and emotional problems in boys with Duchenne's muscular dystrophy: Parental confidence and perception. Child Care Health Developm 1999;5(4):331-338.

34. Hinton V, De Vivo D, Fee R, Goldstein E, Stern Y. Investigation of poor academic achievement in children with Duchenne muscular dystrophy. Learn Disabil Res Pract 2004;19(3):146-154.

35. Hinton VJ, Nereo NE, Fee RJ, Cyrulnik SE. Social behavior problems in boys with Duchenne muscular dystrophy. J Dev Behav Pediatr 2006;27(6):470-476.

36. Hinton VJ, Fee RJ, De Vivo DC, Goldstein E. Poor facial affect recognition among boys with Duchenne muscular dystrophy. J Autism Dev Disord 2007;37(10):1925-1933, doi:10.1007/s10803-006-0325-5.

37. Donders J, Taneja C. Neurobehavioral characteristics of children with Duchenne muscular dystrophy. Child Neuropsychol 2009;15(3):295-304.

38. Donald KA, Mathema H, Thomas KG, Wilmshurst JM. Intellectual and behavioral functioning in a South african cohort of boys with duchenne muscular dystrophy. J Child Neurol 2011;26(8):963-969.

39. Fee RJ, Hinton VJ. (2011). Resilience in children diagnosed with a chronic neuromuscular disorder. J Dev Behav Pediatr 2011;32(9):644-650.

40. Bray P, Bundy AC, Ryan MM, North KN, Everett A. Health-related quality of life in boys with Duchenne muscular dystrophy: Agreement between parents and their sons. J Child Neurol 2010;25(10):11881194.

41. McDonald CM, McDonald DA, Bagley A, Sienko Thomas S, Buckon CE, Henricson E, et al. Relationship between clinical outcome measures and parent proxy reports of health-related quality of life in ambulatory children with Duchenne muscular dystrophy. J Child Neurol 2010;25(9):1130-1144. 
42. Davis SE, Hynan LS, Limbers CA, Andersen CM, Greene MC, Varni JW, et al. The PedsQL in pediatric patients with Duchenne muscular dystrophy: feasibility, reliability, and validity of the Pediatric Quality of Life Inventory Neuromuscular Module and Generic Core Scales. J Clin Neuromusc Dis 2010;11(3): 97-109.

43. Hu J, Jiang L, Hong S, Cheng L, Kong M, Ye Y. Reliability and validity of the Chinese version of the Pediatric Quality Of Life InventoryTM (PedsQLTM) 3.0 neuromuscular module in children with Duchenne muscular dystrophy. Health Qual Life Outcomes 2013;11:47.

44. Lim Y, Velozo C, Bendixen RM. The level of agreement between child self-reports and parent proxyreports of health-related quality of life in boys with Duchenne muscular dystrophy. Quality of Life Research 2014;23(7):1945-1952.

45. Wei Y, Speechley KN, Zou G, Campbell, C. Factors Associated with Health-Related Quality of Life in Children with Duchenne Muscular Dystrophy. J Child Neurol 2015;31(7):879-886.

46. Messina S, Vita GL, Sframeli M, Mondello S, Mazzone E, D'Amico A, et al. Health-related quality of life and functional changes in DMD: A 12-month longitudinal cohort study. J Neuromuscol Disord 2016;26(3):189-196.

47. Wei Y, Speechley KN, Zou G, Campbell C. The relationship between quality of life and health-related quality of life in young males with Duchenne muscular dystrophy. Dev Med Child Neurol 2017;59(11):1152-1157.

48. Landfeldt E, Lindgren P, Bell CF, Guglieri M, Straub V, Lochmuller H, et al. Health-related quality of life in patients with Duchenne muscular dystrophy: a multinational, cross-sectional study. Dev Med Child Neurol 2016;58(5):508-515.

49. Baiardini I, Minetti C, Bonifacino S, Porcu A, Klersy C, Petralia P, et al. Quality of life in Duchenne muscular dystrophy: the subjective impact on children and parents. J Child Neurol 2011;26(6):707-713.

50. Bray P, Bundy AC, Ryan MM, North KN, Burns J. Health status of boys with Duchenne muscular dystrophy: A parent's perspective. J Paediatr Child Health 2011;47(8):557-562.

51. Connolly AM, Florence JM, Cradock MM, Eagle M, Flanigan KM, McDonald CM, et al. One year outcome of boys with duchenne muscular dystrophy using the bayley-III scales of infant and toddler development. Pediatr Neurol 2014;50(6):557-563.

52. Houwen-van Opstal SL, Jansen M, van Alfen N, de Groot IJ. Health-related quality of life and its relation to disease severity in boys with Duchenne muscular dystrophy: satisfied boys, worrying parents--a casecontrol study. Journal of Child Neurology 2014;29(11):1486-1495.

53. Zamani G, Heidari M, Azizi Malamiri R, Ashrafi MR, Mohammadi M, Shervin Badv R, et al. The quality of life in boys with Duchenne muscular dystrophy. J Neuromusc Disord 2016;26(7):423-427.

54. Harper DC. Personality correlates and degree of impairment in male adolescents with progressive and nonprogressive physical disorders. J Clin Psychol 1983;39(6):859-867.

55. Reid DT, Renwick RM. Preliminary Validation of a New Instrument to Measure Life Satisfaction in Adolescents with Neuromuscular Disorders. Int J Rehabil Res 1994;17(2):184-188.

56. Reid DT, Renwick RM. Relating familial stress to the psychosocial adjustment of adolescents with Duchenne muscular dystrophy. Int J of Rehabil Res 2001;24(2):83-93.

57. Simon VA, Resende MB, Simon MA, Zanoteli E, Reed UC. Duchenne muscular dystrophy: quality of life among 95 patients evaluated using the Life Satisfaction Index for Adolescents. Arq Neuropsiquiatr 2011;69(1):19-22.

58. Graham CD, Rose MR. What explains high life satisfaction in men living with Duchenne muscular dystrophy? A preliminary study to inform psychological intervention. Muscle and Nerve 2017;56(1):163-166.

59. Otto C, Steffensen BF, Hojberg AL, Barkmann C, Rahbek J, Ravens-Sieberer U, et al. Predictors of HealthRelated Quality of Life in boys with Duchenne muscular dystrophy from six European countries. J Neurol 2017;264(4):709-723.

60. Landfeldt E, Mayhew A, Straub V, Lochmüller H, Bushby K, Lindgren P. (2018). Psychometric analysis of the pediatric quality of life inventory 3.0 neuromuscular module administered to patients with duchenne muscular dystrophy: A rasch analysis. Muscle and Nerve 2018;58(3):367-373. 
61. Bendixen RM, Senesac C, Lott DJ, Vandenborne K. Participation and quality of life in children with Duchenne muscular dystrophy using the International Classification of Functioning, Disability, and Health. Health Qual Life Outcomes 2012;10:43.

62. Elsenbruch S, Schmid J, Lutz S, Geers B, Schara U. Self-reported quality of life and depressive symptoms in children, adolescents, and adults with duchenne muscular dystrophy: A cross-sectional survey study. Neuropediatrics 2013;44(5):257-264.

63. Pangalila RF, van den Bos GA, Bartels B, Bergen MP, Kampelmacher MJ, Stam HJ, et al. (2015b). Quality of life of adult men with Duchenne muscular dystrophy in the Netherlands: implications for care. J Rehabil Med 2015;47(2):161-166.

64. Lue YJ, Chen SS, Lu YM. Quality of life of patients with Duchenne muscular dystrophy: from adolescence to young men. Disabil Rehabil 2017;39(14):1408-1413.

65. Pangalila RF, van den Bos GA, Bartels B, Bergen M, Stam HJ, Roebroeck ME. Prevalence of fatigue, pain, and affective disorders in adults with duchenne muscular dystrophy and their associations with quality of life. Arch Phys Med Rehabil 2015;96(7):1242-1247.

66. Achenbach TM. Manual for the Child Behavior Checklist/4-18 and 1991 profile. University of Vermont, Department of Psychiatry, 1991.

67. Cook DA, Beckman TJ. (2006). Current concepts in validity and reliability for psychometric instruments: theory and application. Am J Med 2006;119(2):166-e7.

68. American Psychiatric Association. Diagnostic and statistical manual of mental disorders (DSM- $\left.5^{\circledR}\right)$. American Psychiatric Pub, 2013.

69. Goodman R, Scott S. (1999). Comparing the Strengths and Difficulties Questionnaire and the Child Behavior Checklist: is small beautiful? J Abnorm Child Psychol 1999;27(1):17-24.

70. Gianarris WJ, Golden CJ, Greene L. The Conners'Parent Rating Scales: A critical review of the literature. Clin Psychol Rev 2001;21(7):1061-1093.

71. Hall CL, Guo B, Valentine AZ, Groom MJ, Daley D, Sayal K, Hollis C. The validity of the Strengths and Difficulties 27.Questionnaire (SDQ) for children with ADHD symptoms. PloS One 2019;14(6):e0218518.

72. Kroenke K, Spitzer RL, Williams JB, Monahan PO, Löwe B. Anxiety disorders in primary care: prevalence, impairment, comorbidity, and detection. Ann Intern Med 2007;146(5):317-325.

73. Spitzer RL, Kroenke K, Williams JB, Löwe B. A brief measure for assessing generalized anxiety disorder: the GAD-7. Arch Intern Med 2006;166(10):1092-1097.

74. Kroenke K, Spitzer RL, Williams JB, Löwe B. The patient health questionnaire somatic, anxiety, and depressive symptom scales: a systematic review. General Hospital Psychiatry 2010;32(4):345-359.

75. Bourdon KH, Goodman R, Rae DS, Simpson G, Koretz DS. (2005). The Strengths and Difficulties Questionnaire: US normative data and psychometric properties. J Am Acad Child Adolescent Psychiatry 205;44(6), 557-564.

76. Goodman R. Psychometric properties of the strengths and difficulties questionnaire. J Am Acad Child Adolesc Psychiatry 2001;40(11):1337-1345.

77. Hysing M, Elgen I, Gillberg C, Lie SA, Lundervold AJ. Chronic physical illness and mental health in children. Results from a large-scale population study. J Child Psychol Psychiatry 2007;48(8):785-792.

78. Hysing M, Elgen I, Gillberg C, Lundervold AJ. Emotional and behavioural problems in subgroups of children with chronic illness: results from a large-scale population study. Child Care Health Dev 2009;35(4):527-533.

79. Klasen H, Woerner W, Rothenberger A, Goodman R. German version of the Strength and Difficulties Questionnaire (SDQ-German)--overview and evaluation of initial validation and normative results. Prax Kinderpsychol Kinderpsychiatr 2003;52(7):491-502.

80. Smedje H, Broman JE, Hetta J, Von Knorring AL. Psychometric properties of a Swedish version of the "Strengths and Difficulties Questionnaire". Eur Child Adolesc Psychiatry 1999;8(2):63-70.

81. Bray P, Bundy AC, Ryan MM, North KN. Feasibility of a computerized method to measure quality of "everyday" life in children with neuromuscular disorders. Phys Occup Ther Pediatr 2010;30(1):43-53.

82. Fowler WM Jr, Abresch RT, Koch TR, Brewer ML, Bowden RK, Wanlass RL. (1997). Employment profiles in neuromuscular diseases. Am J Phys Med Rehabil 1997;76(1):26-37. 
83. Wilson S, Washington LA, Engel JM, Ciol MA, Jensen MP. Perceived social support, psychological adjustment, and functional ability in youths with physical disabilities. Rehabil Psychol 2006;51(4): 322-330.

84. Dany A, Barbe C, Rapin A, Reveillere C, Hardouin JB, Morrone I, et al. Construction of a Quality of Life Questionnaire for slowly progressive neuromuscular disease. Qual Life Res 2015;24(11):2615-2623.

85. Dany A, Rapin A, Lavrard B, Saout V, Reveillere C, Bassez G, et al. The quality of life in genetic neuromuscular disease questionnaire: Rasch validation of the French version. Muscle Nerve 2017;56(6):1085-1091.

86. Orcesi S, Ariaudo G, Mercuri E, Beghi E, Rezzani C, Balottin U. A new self-report quality of life questionnaire for children with neuromuscular disorders: Presentation of the instrument, rationale for its development, and some preliminary results. J Child Neurol 2014;29(2):167-181.

87. Whitcomb SA. Behavioral, social, and emotional assessment of children and adolescents. Routledge, 2013.

88. Hoza B. Peer functioning in children with ADHD. J Pediatr Psychol 2007;32(6):655-663.

89. Pine DS, Cohen P, Gurley D, Brook J, Ma Y. (1998). The risk for early-adulthood anxiety and depressive disorders in adolescents with anxiety and depressive disorders. Arch Genet Psychiatry 1998;55(1):56-64

90. Woodward LJ, Fergusson DM. (2001). Life course outcomes of young people with anxiety disorders in adolescence. J Am Acad Child Adolesc Psychiatry 2001;40(9)C:1086-1093. 
<smiles>Cc1ccccc1</smiles> 


\section{Chapter 7}

Computerized working memory training in males with

Duchenne muscular dystrophy: a single case experimental design study

Danique M.J. Hellebrekers, Jose M.A. Wirken, Judith M. Lionarons, Sander M.J. van Kuijk, Sylvia Klinkenberg, Johan S.H. Vles, Jos G.M. Hendriksen Revisions Neuropsychological Rehabilitation 


\section{Abstract}

Learning disabilities (LDs) and working memory problems (WM) are common brainrelated comorbidities in Duchenne muscular dystrophy (DMD). Despite growing evidence on the efficacy of computerized WM training in children with LDs, research in DMD is lacking. This study assessed whether (1) computerized training improves dystrophin-associated verbal WM problems of DMD patients, (2) effects of training are present immediately at post-intervention, at 3 and 8 months follow-up and (3) training improves problems that arise from their LDs. Four DMD patients with LDs and WM problems completed multiple cognitive tests of verbal and visual WM, academics, attention, processing speed and fluid reasoning. Parents and teachers completed executive behavioural questionnaires. Tests and questionnaires were administered at baseline, post-intervention, 3 and 8 months follow-up. A single case experimental design (SCED) additionally evaluated decreases in WM problems of patients based on parent reports. Short and long-term, near-and far transfer effects were found for verbal and visual WM, academics, processing speed and fluid reasoning. Parent and teacher questionnaires showed minimal changes. SCED results showed positive effects on WM. Promising WM training results are shown in DMD that merit further research. SCED is a useful methodology for DMD intervention studies and gives valuable information on cognitive changes. 


\section{Introduction}

WM is a limited capacity system that temporarily stores, rehearses and processes internally-held information. ${ }^{1}$ It is regulated by several (sub)cortical and cerebellar regions. $^{2-4}$ WM has an important role in the guidance of everyday behaviour and in performing complex tasks such as learning, comprehension, reasoning and planning. ${ }^{1,5,6}$ Traditionally, WM was viewed as a fixed system, but there is increasing evidence that it can be improved by educational support and interventions such as (computerized) WM training. ${ }^{7-12}$ Training of WM increases brain activity in (sub)cortical areas e.g. in the basal ganglia and frontoparietal regions and networks. ${ }^{2-4}$ The effect of WM training on neuronal bases remains poorly understood. However, changes in white matter structure (increased myelination) and dopamine D1 receptor density have been demonstrated recently. ${ }^{2,12}$ Transfer of WM training can be evaluated by assessing near effects (WM related abilities) and far effects (other cognitive abilities, academic skills and behavioural changes). Reliable near and far transfer effects have been described in children with learning disabilities (LDs) or attention-deficit hyperactivity (ADHD) disorders, particularly in the older ones ( $\geq 10$ year) ${ }^{9-11,13,14}$ Short and long-term near and far transfer effects are found for verbal and visuospatial WM, academic skills (reading, arithmetic abilities, spelling, word decoding) and fluid reasoning. ${ }^{5,7,9-11,13,15,16}$ Studies using the computerized program Jungle Memory $^{\mathrm{TM}}$ displayed the highest effects, especially in visuo-spatial abilities. ${ }^{9}$ It is valuable to evaluate whether remediation tools as (computerized) WM training are also beneficial for patients with Duchenne muscular dystrophy (DMD) and a comorbid LD with WM deficiencies.

The recessive X-linked neuromuscular disorder $\mathrm{DMD}$, is caused by mutations in the dystrophin-encoding $(D M D)$ gene leading to functional loss of the full-length dystrophin protein (Dp427) in various tissues including muscles and the brain. ${ }^{17} \mathrm{~A}$ lack of dystrophin in muscles causes progressive muscle weakness, which eventually leads to fatal respiratory and cardiac complications. ${ }^{18,19}$ In the brain, dystrophin (Dp427 B $^{\text {) is }}$ localized in the hippocampus, amygdala and the cerebral cortex with a slightly higher expression in the temporal and frontal cortex than in the parietal and occipital cortex. ${ }^{20-23}$ In addition to $\mathrm{Dp} 427_{\mathrm{B}}$, shorter brain isoforms exist i.e. Dp140 and Dp71/Dp40. ${ }^{20,24,25}$ Depending on the mutation location in the $D M D$-gene the expression of these shorter isoforms are prohibited. Deficiencies of the brain isoforms result in functional and structural alterations in neurons and glial cells and these have been related to the presence of non-motor comorbidities in patients with DMD including neurocognitive impairments, behavioural disorders and epilepsy. ${ }^{20,21}$ For instance in hippocampal neurons dystrophin alters $\gamma$-aminobutyric acid type $A\left(G A B A_{a}\right)$ receptor 
functions at inhibitory synapses and glutamergic functions at excitatory synapses. ${ }^{20,21,26}$ The malformations and dysfunctions of synapses in crucial neural networks of the hippocampus, likely induce the core impairments in verbal WM or span capacity of patients with DMD. ${ }^{26}$ WM deficiencies have been related to the frequently observed LDs in DMD. ${ }^{27-32}$ High prevalence rates of verbal LDs such as dyslexia or reading disabilities and non-verbal LDs for instance dyscalculia or arithmetic disabilities have been found. ${ }^{29,33-36}$ Reading impairments for example are reported in up to $40 \%$ of males with DMD. ${ }^{27,33-36}$ Due to the deficient dystrophin effects on neuronal structures and networks involved in cognition (e.g. WM) one might expect that WM training is less effective in DMD patients. Thereby, this study aimed to explore whether (1) computerized training may also improve WM in DMD patients with dystrophinassociated verbal WM problems, (2) effects of the computerized WM training are present immediately at post-intervention and remain at 3 and 8 months follow-up (FU) and (3) WM training improves problems that arise from the comorbid LDs of patients with DMD.

\section{Materials and methods}

\section{Participants and procedure}

Five male patients with a genetically confirmed diagnosis of DMD attending the outpatient clinic of Kempenhaeghe, Centre for Neurological Learning Disabilities (CNL), Heeze, The Netherlands were asked to participate. Patients had comorbid LDs including reading or arithmetic disabilities, dyslexia, dyscalculia and/or ADHD, which were previously diagnosed by health professionals. Between September 2016 and October 2017, these patients underwent an extensive re-evaluation of cognitive and behavioural functioning on the indication of regular clinical care at CNL. Thereafter, patients were approached by the child neuropsychologist (JH) and child neurologist (SK) for participation in the current study. Inclusion criteria were: (1) age of 10-16 years, (2) intellectual quotient (IQ) score of $\geq 70$ to exclude mental retardation, (3) WM problems indicated by $z$-scores $\geq-1$, (4) intact physical mobility of upper extremities represented by a Brook score $\geq 5$, (5) no hearing or vision problems that could interfere with the computerized training or neurocognitive testing, (6) undergoing no other psychological or cognitive interventions, (7) no use of psychostimulants, and (8) having an adequate proficiency in Dutch. Ethical approval was given by Kempenhaeghe Ethics Committee (project number 18.04). Written informed parental and patient consent 
were obtained. The study was conducted in accordance with the $18^{\text {th }}$ World Medical Assembly, Helsinki 194.

\section{Study design}

The present study consisted of different testing phases (see Figure 7.1). At baseline (T1) patients completed a short battery to estimate IQ, verbal and visual working memory, sustained attention, processing speed and academic skills (reading and arithmetic). In addition, parents and teachers completed questionnaires on executive functioning (Q1). After T1 and Q1, patients and parents jointly received psycho-education on WM and its relation to learning and academic development. Additionally, after T1 a single case experimental design (SCED) i.e. a multiple baseline across patients method was established (see Figure 7.1). The SCED consisted of a baseline period (phase A1), intervention period (phase B1) and follow-up period (phase A2). The baseline period (phase A1) of the SCED, started directly after the baseline cognitive assessment (T1) and the psycho-education. Phase A1, lasted a minimum of three and maximum of nine weeks depending on randomisation outcomes (see Figure 7.1). Simple randomisation (digital dice-throwing) determined whether the patient started with the intervention (Phase B1) in week $4,5,6,7,8$ or 9 (see Figure 7.1). Each dice number represented a starting week (e.g. dice number one represented start week 4). The intervention lasted eight weeks in total and took place between week 4 to 17 (see Figure 7.1). After the intervention, the follow-up period started (Phase A2) between week 12 to 20, which lasted three weeks in total (see Figure 7.1). In addition, patients underwent neurocognitive testing (T2) at home and completed the same cognitive battery as at $\mathrm{T} 1$, without IQ estimation (see Figure 7.1). At 3 (T3) and 8 months (T4) follow-up, patients completed the same short cognitive battery of T2 at home (see Figure 7.1). Furthermore, at T2, T3 and T4, parents and teachers completed the same questionnaires on executive functioning as at T1 (see Figure 7.1).

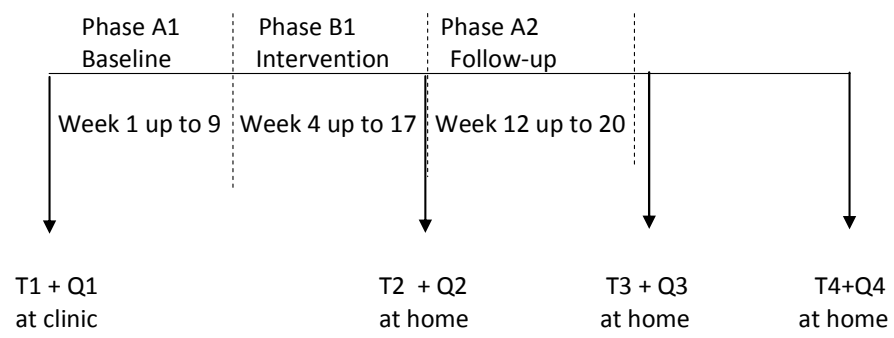

Figure 7.1 Study design. T=neurocognitive testing, $Q=$ =questionnaires for parents and teachers, Phase $\mathrm{A} 1=$ baseline period with a minimum of 3 weeks and maximum of 9 weeks; Phase B1 = intervention period of 8 weeks; Phase $A 2$ = follow-up period of maximum three weeks. 


\section{Measures}

Demographic and disease-related parameters were extracted from the electronic patient files. Extracted parameters were age, educational level (i.e. regular or special education), handedness, DMD gene mutation, comorbid LD diagnose, IQ and memory scores of previous neurocognitive testing between September 2016 to October 2017.

\section{Cognitive and academics testing at $T 1, T 2, T 3$, and $T 4$}

General intellectual functioning was estimated using the Peabody Picture Vocabulary test-III (PPVT-III-NL), which is a commonly used non-motor test in DMD. ${ }^{28,37}$ Raw scores were standardized to age-related norm scores (Mean $=100, S D=15) .{ }^{37}$ Subtests of the Wechsler Intelligence Scale for Children-fifth edition (WISC-V-NL) were used to assess verbal working memory (Digit span and Letter-Number Sequencing), visual working memory (Picture span), fluid reasoning (Matrix Reasoning) and processing speed (Symbol Search). ${ }^{38}$ Test-retest reliability of the subtests vary from moderate to high (Digit span $=.79$, Letter-Number sequencing $=.68$, Picture span $=.60$, Matrix Reasoning $=.53$ and Symbol search $=.73) .{ }^{38}$ Sustained attention was tested by the subtest Score! of the Test of Everyday Attention for Children, Second Edition (TEA-Ch-II) and its testretest reliability is moderate (.64). ${ }^{39,40}$ Technical reading was evaluated by the Dutch Continu Benoemen \& Woorden Lezen (CB\&WL) test using the subtests monosyllabic word reading and the EMTB-T50 score. Words of these subtests are not coherent and differ in length and complexity. Based on the EMTB-T50 score a one-minute reading score was calculated as well. Raw scores of the reading tests were based on the total number of words read correctly. Test-retest reliability of the reading subtests are high (range: .90-.91). ${ }^{41}$ Raw scores of the WISC-V, TEA-Ch and CB\&WL were transformed to standardized scaled scores (Mean=10, $S D=3$ ), based on age-adjusted norm data as specified within the test manuals. ${ }^{38,39,41}$ The Tempo Test Automatization (TTA) was used to evaluate the degree of memorization of mathematical facts. The TTA consists of four pages of 50 arithmetic problems including one page of addition problems, subtraction problems, multiplication problems and one page of division problems. ${ }^{42}$ Scores are based on total number of arithmetic problems answered correctly (range 0-50). ${ }^{42}$ The addition and subtraction scores can be computed to calculate a first total score (tta). Scores of all four subtests result in a second total score (TTA). Test-retest reliability of the total TTA scores are high (range: .82-.83). ${ }^{42}$

\section{Behavioural testing at $T 1, T 2, T 3$, and T4}

The Behaviour Rating Inventory for Executive Functions questionnaire for parents (BRIEF-P) and for teachers (BRIEF-T) evaluated executive functioning based on two 
indices: Metacognition index $(\mathrm{MCl})$ and Behavioural Regulation Index (BRI). ${ }^{43}$ The two indices are assessed by eight subscales: Inhibit, Shift, Emotional control, Initiate, Working Memory, Plan-organize, Organization of Materials and Monitor. A global executive score (GEC) can be computed based on the $\mathrm{MCl}$ and $\mathrm{BRI}$ outcomes. ${ }^{43}$ Parents and teachers rated items using a three-point Likert scale ( $1=$ never a problem to $3=$ often a problem).$^{43}$ Higher scores reflected greater difficulties in executive behaviour and abilities. Previous studies described high test-retest reliability for the BRIEF-P for $\mathrm{MCI}(I C C=.84)$, BRI (ICC=.95) and GEC (ICC=.86), and for the BRIEF-T for $\mathrm{MCI}(I C C=.74)$, $\mathrm{BRI}(\mathrm{ICC}=.76)$ and GEC $(I C C=.77) .{ }^{43}$ Raw scores of the BRIEF-P and BRIEF-T were transformed to age-adjusted normative data ( $T$-scores with Mean=50 and SD=10). ${ }^{43}$

\section{SCED: Multiple baseline design measurement}

Throughout the baseline period (Phase A1), the intervention period (Phase B1) and the follow-up period (Phase A2) parents digitally completed the WM subscale of the BRIEF$P$. The 10-item WM subscale consisted of questions such as "my son has troubles with chores or tasks that have more than one step" or "my son has troubles remembering things, even for a few minutes" ${ }^{43}$ Items were digitally rated on a three-point Likert scale from: 1=never to $3=$ often, with higher scores representing more difficulties with WM. $^{43}$ The minimum total score of this ten-item WM subscale was 10 and the maximum score was 30 . It has previously been proven that the internal consistency and test-retest reliability of this subscale is high (Cronbach's alpha $=.90$, ICC $=.73$ ). ${ }^{43}$

\section{Psychoeducation}

Patients and parents jointly received standardised psycho-education on the role of WM problems in daily life during visits at the outpatient clinic of CNL. The information was given by psychologists who were trained Jungle Memory coaches (DH/JW). The following aspects were explained: 1) what is WM? 2) when do you use your WM? 3) what is the importance of good WM? 4) what happens when you have WM problems and (5) what is the goal of the computerized Jungle Memory ${ }^{\mathrm{TM}}$ training and how does this program work? These aspects were written down in a WM sketchbook that was specifically developed for the patients of the current study. This workbook also consisted of pen and paper games on the abovementioned aspects. For example, patients had to read and rehearse information, make puzzles, or perform dual-tasks to learn the functions of WM. 


\section{Intervention}

Jungle Memory ${ }^{\mathrm{TM}}$ (2008) is a web-based WM training program aimed at children aged 7-16 years. ${ }^{11}$ This program comprises of three interactive computer games with up to 30 levels of difficulty in each game. Different aspects of WM are trained during each game and children are provided with regular feedback on their progress. Game 1 (Quicksand) involves memory for and later use of word endings, game 2 (Code Breaker) involves mental rotation of letters and game 3 (River Crossing) involves sequential memory of mathematical solutions. ${ }^{11}$ The program includes motivational features such as positive verbal feedback, display of patients scores, percentile rankings and the number of monkey's collected as a result of successful completion of training levels. ${ }^{11}$ Each game consisted of a maximum of ten trials per training session, leading to a total performance of 30 trials on each day of training. Each training session lasted approximately 15-20 minutes. Patients in the current study used the program four times a week leading to 96 sessions ( 32 sessions per game) that were completed over an eight-week period of training. Treatment fidelity was monitored by (1) parent supervision, (2) weekly sessions with the Jungle Memory coaches (DH/JW), and (3) log data that was generated from the training program. Each time a patient logged in and completed all 10 trials for the three games, the program recorded their participation in a database log. The Jungle Memory coaches (DH/JW) weekly reviewed the log files to verify compliance of patients.

\section{Statistical analyses}

Demographic, disease-related characteristics and previous neuropsychological testing results on memory of patients were analysed using descriptive statistics. Age-related norm data of the cognitive and behavioural tests were transformed to z-scores (mean=0, SD=1). Reliable change indices $(\mathrm{RCl})$ were calculated to detect clinical significant individual changes on cognitive and behavioural outcomes between 1 : baseline (T1) versus post-intervention (T2), 2: baseline (T1) versus three months followup (T3), 3: baseline (T1) versus eight months follow-up (T4). An $R C l$ value of $z=-1.96$ or $z=+1.96$ was used as criterion for a clinical significant change. ${ }^{44,45}$

For SCED, the study size was determined on the following formula: $p=1 /\left(6^{*} 6 * 6\right)=0.005$, reflecting that effects found attribute to $p<.05 .{ }^{46}$ This calculation is based on the random assignments of at least $n=3$ patients who could start with the intervention at six different time-points, namely at week 4,5,6,7,8 and 9. Data of the SCED (multiple baseline measurements) were plotted graphically using $R$ to visually assess a change in WM outcomes during and after the intervention. In addition, Tau- $U$ weighted effect sizes were calculated to determine significant clinical differences between phase $A 1$, 
phase $\mathrm{B} 1$ and phase $\mathrm{A} 2$ for each patient (i.e. phase contrast differences). Tau-U scores ranged from -1 to +1 , with positive scores indicating more WM difficulties and negative scores reflecting less WM difficulties. Tau-U effect sizes were defined as: $0-0.62=$ small, $0.63-0.92=$ moderate and $0.93-1.00=$ strong. ${ }^{47,48}$ To calculate Tau- $U$ weighted effect sizes an online calculator was used: www.singlecaseresearch.org/calculators/tau-u. ${ }^{47,48}$ P-values at the .05 and .01 (two-sided) were considered statistically significant.

\section{Results}

\section{Training compliance}

Five patients were included of which four completed the entire study. One patient dropped out during the last week of the intervention period, because he was admitted to the hospital for medical problems. The eight-week training period of the four remaining patients was not interrupted by holidays or other activities. The database log for compliance showed that only case 2 trained twice instead of four times at his second week of the intervention, for which we contacted parents to inquire whether they experienced any problems during the training. No problems were reported and parents likely inappropriately closed the training sessions.

The characteristics of the four included patients are displayed in Table 7.1. Three patients (case 2, 3 and 4) visited special education and one (case 1) visited regular education, but received special educational support for his LD. The included patients had reading disabilities, arithmetic disabilities and WM problems (z-scores ranged from -1.3 to -2.3 ; see Table 7.1). Two patients (case 2-4) were also diagnosed with ADHD. Previous neuropsychological data on long-term memory was available of three patients (case $1,3,4$ ) of which none displayed deficits in this domain.

Table 7.1 Patient characteristics.

\begin{tabular}{llllcrccc}
\hline Case & Age & Genetic mutation & Handedness & Education & IQ & $\begin{array}{c}\text { Brooke } \\
\text { score }\end{array}$ & $\begin{array}{c}\text { Working } \\
\text { memory }\end{array}$ & $\begin{array}{c}\text { Long-term } \\
\text { memory }\end{array}$ \\
\hline 1 & 12,4 & Deletion exon 22-44 & $\mathrm{R}$ & Regular & 84 & 3 & -1.3 & -0.5 \\
2 & 11,2 & Deletion exon 51-54 & $\mathrm{R}$ & Special & 95 & 1 & -1.5 & - \\
3 & 15,7 & Deletion exon 45-52 & $\mathrm{R}$ & Special & 94 & 2 & -1.9 & +1.3 \\
4 & 12,1 & Deletion exon 52 & $\mathrm{L}$ & Special & 101 & 3 & -2.3 & -0.8 \\
\hline
\end{tabular}

Working memory and long-term memory outcomes are $\mathrm{z}$-scores, $\mathrm{IQ}=$ =intelligence quotient score, $\mathrm{R}=$ right, $\mathrm{L}=$ left. Brooke scores ranges from 1-6 (1=intact arm/hand function and $6=$ no useful function of hands). 


\section{Evaluation and feedback of patients and parents}

The eight-week intervention period was time-consuming and regularly frustrated patients when training levels increased in difficulty. Though their overall experience was positive. Case 1, 3 and 4 experienced improvements in daily life functioning i.e. at home or school during and after the intervention. For instance case 1 and case 4 noticed faster completion of school exercises, whereas case 3 reported less wordfinding difficulties during spontaneous conversations.

\section{Cognitive and academic testing outcomes}

Table 7.2 displays the outcomes of the multiple cognitive testing measurements. Baseline (T1) results showed that the estimated intelligence indicated by the PPVT-IIINL ranged between 83-108 (see Table 7.2). At post-intervention (T2), results showed reliable improvements on simple verbal working memory task (Digit span; $n=1$ ), dualtasks of verbal working memory (Letter-Digit span; $n=2$ ), academic skills of reading $(n=4)$ and arithmetic $(n=1)$, processing speed $(n=4)$ and fluid reasoning $(n=1)$, see Table 7.2. At 3 months follow-up (T3), reliable improvements were still found on dual-task of verbal working memory $(n=1)$, academic skills of reading $(n=3)$ and arithmetic $(n=1)$, processing speed $(n=4)$ and fluid reasoning $(n=1)$, see Table 7.2. In addition, one patient (case 1 ) showed improved performances on visual working memory when his T3 outcome was compared to his baseline performance, but this improvement was not found at T2. At 8 months follow-up (T4), reliable improvements were yet found on dual-task of verbal working memory $(n=1)$, academic skills of reading $(n=2)$ and arithmetic $(n=1)$, processing speed $(n=4)$ and fluid reasoning $(n=1)$, see Table 7.2. Furthermore, on dual-tasks of verbal working memory two patients (case 1 and 4) performed better at T4 compared to T1, but this effect was not noticed at T2 or T3. Some patients also displayed reliable significant decreased performances instead of improvements on visual working memory (case 2) and arithmetic (case 1 and case 3), see Table 7.2. For case 2, no total math score could be calculated because he was not able to complete divisions due to his LD. On T2, T3, and T4, all cases showed no reliable changes on sustained attention. 


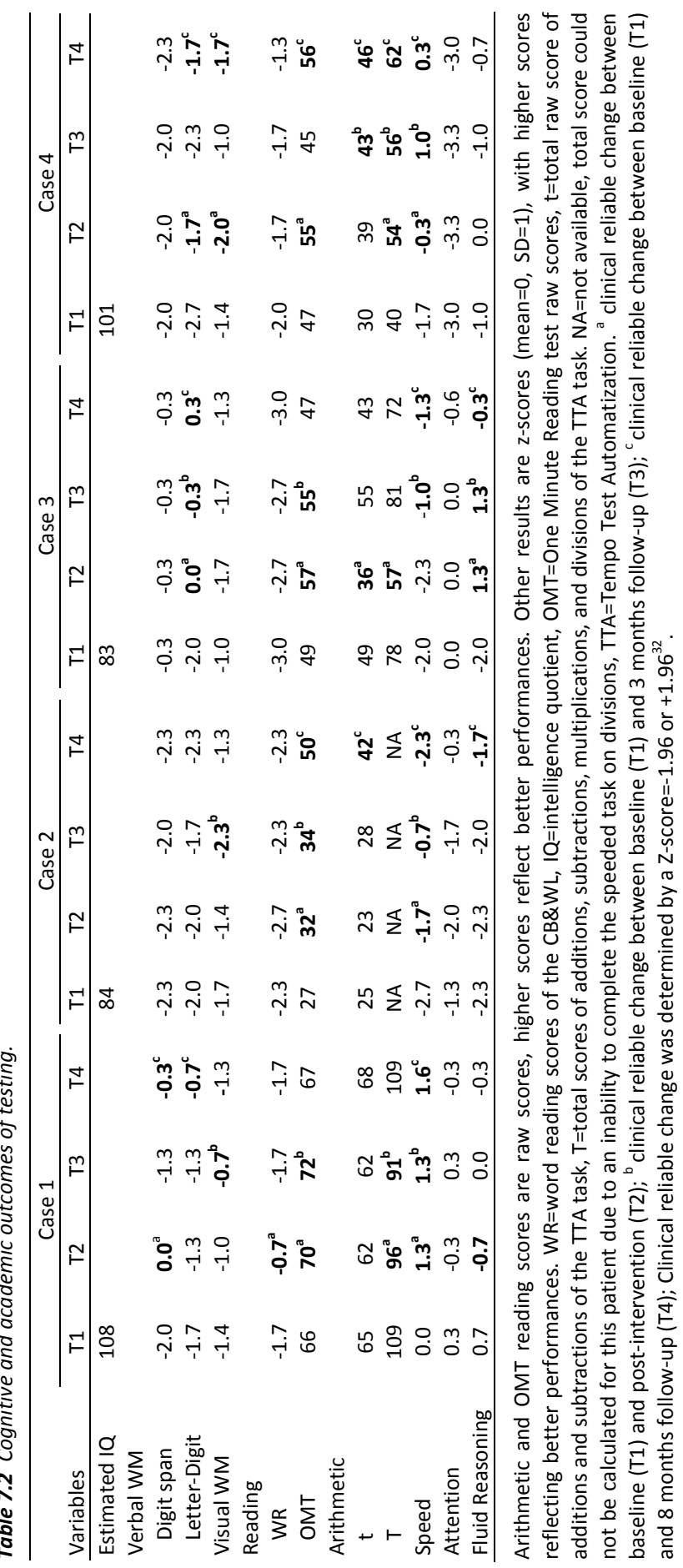




\section{Behavioural testing outcomes}

Table 7.3 displays the scores of the multiple behavioural testing measurements. Results of the testing showed reliable improvements on the BRIEF subscale $\mathrm{MCl}$ and total BRIEF GEC score at post-intervention (T2) based on parent reports in one patient (case 2; see Table 7.3). At 3 months follow-up (T3), reliable changes on the subscales $\mathrm{MCl}, \mathrm{BRI}$ and total score GEC were documented for case 2 (see Table 7.3). In addition, improvements on BRI and total score GEC were also reported for case 3 (see Table 7.3). At 8 months follow-up (T4), parent reported changes on $\mathrm{MCl}$ and total GEC were still noticed for one patient (case 2). No reliable changes were reported by teachers for all patients on the BRIEF subscales $\mathrm{MCI}, \mathrm{BRI}$ and the BRIEF total scale GEC (see Table 7.3). 


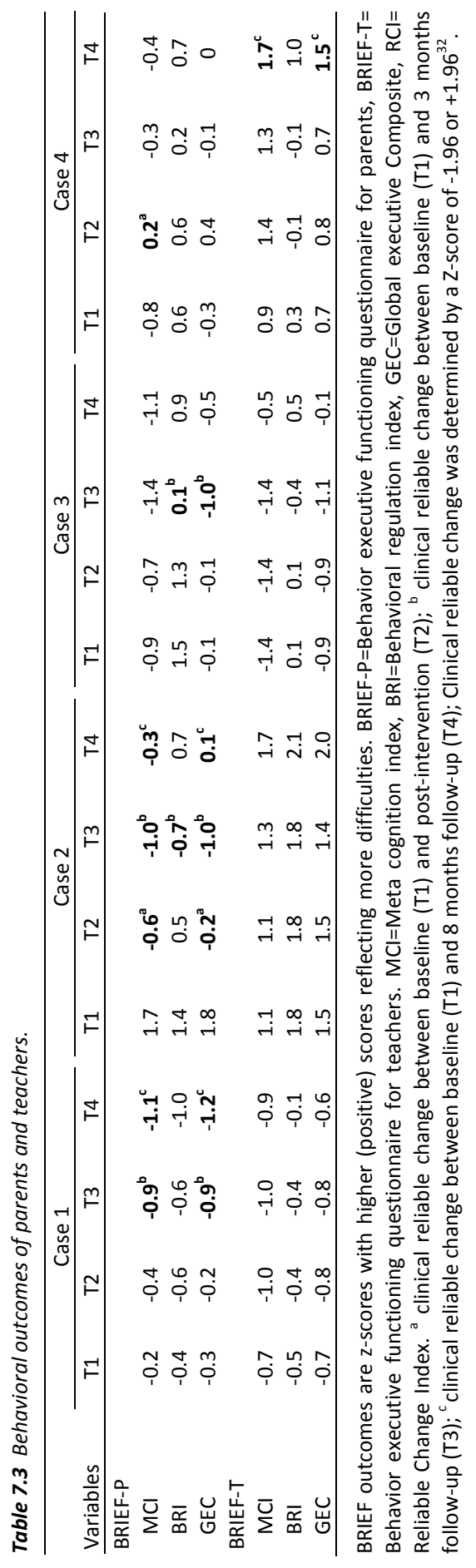


SCED: Multiple baseline design measurement

The items of the BRIEF-working memory subscale were all administered by mothers of patients. Visual analysis showed that working memory functions of case 1 were approximately the same at baseline (Phase A1), during the intervention (Phase B) and after the intervention (A2) (see Figure 7.2). For case 2, case 3 and case 4 we visually observed a change on WM between Phase A1, Phase B and Phase A2 (see Figure 7.2).
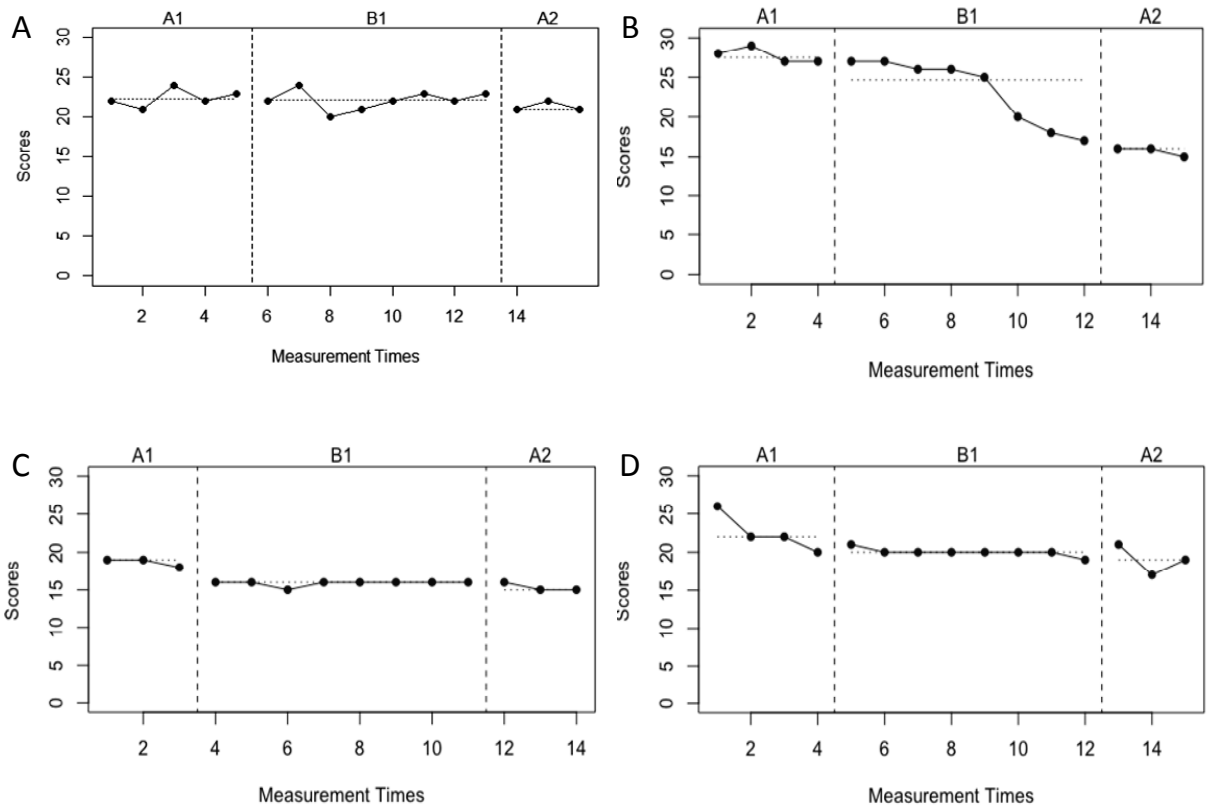

Figure 7.2 A-D Visual analysis of multiple baseline measurement on WM problems reported by parents of included $D M D$ males $(N=4)$. $A 1=$ baseline period, $B 1=$ intervention period, $A 2=$ follow-up. Scores are outcomes of BRIEF Working Memory subscale.

In addition to the visual analysis, Tau- $U$ weighted effect sizes determined significant changes in reported WM problems by parents between the different SCED phases: A1, $B 1$ and A2. Tau- $U$ results showed small effects for case 1 and for case 4, moderate for case 3 and strong effects for case 2, see Table 7.4. 
Table 7.4 Multiple baseline design outcomes.

\begin{tabular}{lcccc}
\hline Case & Tau-U effect size & p-value & \multicolumn{2}{c}{ Cl 95\% } \\
\cline { 4 - 5 } & & & Lower & Upper \\
\hline 1 & -0.26 & .323 & -0.78 & 0.26 \\
2 & -0.93 & $.000^{* *}$ & -1 & -0.40 \\
3 & -0.77 & $.007^{* *}$ & -1 & -0.21 \\
4 & -0.55 & $.044^{*}$ & -1 & -0.14 \\
\hline
\end{tabular}

Results are weighted averages of Tau- $U$ effect sizes, $\mathrm{Cl} 95 \%=95 \%$ confidence intervals. ${ }^{*} p<.05$ (two-sided); $* * p<.01$ (two-sided).

\section{Discussion}

In the present study, we evaluated whether (1) computerized training improves WM in males with DMD who have dystrophin-associated verbal WM problems, (2) effects of the computerized WM training are present immediately at post-intervention or remain at 3 and 8 months follow-up (FU) and (3) WM training improves problems that arise from the comorbid LD of males with DMD. Despite that the intervention was timeconsuming for patients and parents, their overall experience was positive. Neurocognitive testing displayed short and long-term near transfer effects for verbal and visual WM. Short and long-term far transfer effects were found for academic tasks including reading and arithmetic, processing speed and fluid reasoning. Behavioural reports of parents displayed minimal improvements. Outcomes of SCED on WM showed positive small to strong significant effects of the intervention.

\section{Outcomes of cognitive and behavioural testing}

Near transfer effects were found immediately after the intervention for verbal WM in simple and dual-tasks. It is important to note that the features of the stimuli in training tasks were different from those of the verbal and visual WM tests, thus gains in WM are unlikely a consequence of improvements in test-taking skills. In addition, we found far transfer effects for academic skills of reading and arithmetic, as well as processing speed and fluid reasoning. Parents reported minimal improvements and teacher reports showed no improvements in executive behaviour. At 3 and 8 months follow-up, near transfer effects were found for verbal and visual WM. Far transfer effects on academic skills of reading and arithmetic, processing speed and fluid reasoning maintained. Parents reported again minimal improvements and teacher reports displayed no improvements in executive behaviour. Our near transfer effects on WM and far transfer effects in academic skills (e.g. reading, arithmetic abilities, spelling), 
fluid reasoning (e.g. complex reasoning) are in line with previous studies in school-aged children with LDs or ADHD.,10,11,13,15,16 It has been suggested that WM training not solely improves WM related tasks (near effects), but also improves other non-trained domains. For instance, the Jungle Memory ${ }^{\mathrm{TM}}$ program includes no fluid reasoning task, but we found a reliable improvement in fluid reasoning in one patient, which is in line with the study findings of Jaeggi and colleagues. ${ }^{7}$ WM may thus be a domain-general source and an important component for other cognitive functions. ${ }^{8,13,49}$ However, generalizations of improvements to the context of daily life (e.g. at school) should be further investigated, particularly since we found no beneficial improvements reported by teachers. This is similar to the results of Klingberg et al. (2005) and Gray et al. (2012)..$^{13,50}$ Furthermore, the cognitive outcomes of current study varied between patients and differed between testing measurements. For instance, at 3 and 8 months follow-up we noticed improvements that were not found immediately after the intervention. The delayed improvements may be caused by an 'expectancy' effect of patients on beliefs that training should positively influence WM, academic skills and other cognitive abilities. ${ }^{8}$ The delayed follow-up improvements may also be explained by increased self-efficacy or increased motivation. ${ }^{11,51}$ Another explanation for the delayed improvements is that in the meantime patients may have developed strategies such as rehearsal or visualization of information that improved their performances at follow-up. ${ }^{52,53}$ It has been proven that use of memory skills positively impacts speed, efficiency, accuracy, and leads to more confidence in using WM related functions. ${ }^{54}$

\section{SCED outcomes of reported working memory problems}

The SCED visual analysis and Tau-U results of weekly reports on WM problems by parents of patients showed positive small to strong significant effects of the intervention, but parental bias could not be ruled out. Unfortunately, we had no weekly reports of other informants. This could have given valuable information, especially as a discrepancy in ratings between parents of DMD patients and other informants is shown previously. ${ }^{55}$ Future studies should also evaluate reports of other informants i.e. patients or teachers and measure effects on academic skills in addition to WM to evaluate whether the training induce generalizations to school performances.

\section{Efficacy of WM training in DMD}

Despite that we found positive effects of WM training in a relatively small sample it is not clear whether the intervention will result in altered activity, plasticity and synaptic transmission in the subcortical areas and cortical areas which is shown in other populations. ${ }^{2-4,56}$ since in DMD, dystrophin deficiency alters neuronal excitability and 
structural connectivity in hippocampal regions, as well as in the cerebral cortex. Future ( $\mathrm{fMRI}$ ) studies should elucidate whether WM training cause increased activity and alterations in the critical WM networks in patients with DMD. Furthermore, long-lasting effects of training on neuronal excitability and structural connectivity should also be investigated. Since in addition to the loss of neuronal dystrophin, potential contributory factors to DMD-associated cognitive dysfunctions are inflammatory immune mediators (i.e. cytokines such as IL-6, IL-1 $\beta$, and TNF $\alpha$ ). ${ }^{26}$ In muscles chronic inflammation is a key symptom and contributory factor to the pathogenesis of DMD patients. ${ }^{26}$ Inflammatory immune mediators exert neuronmodulatory effects on the hippocampus and have been linked to altered learning and formation of memories. ${ }^{26}$ In this context, one may expect that the effects of WM training on neuronal excitability and structural connectivity are not long-lasting in DMD patients. Yet, there is no evidence that brain levels of neuromodulatory cytokines are altered in DMD. ${ }^{26}$ Nonetheless, our explorative results show comparable near and far transfer effects compared to studies evaluating patient populations with LDs or ADHD without DMD. ${ }^{7,10,11,13,15,16}$ We might speculate that dystrophin may not be involved in WM regions, networks and functions as previously expected. When this is the case similar effects of WM training are expected in patients with partially functional dystrophin, i.e. Becker muscular dystrophy. Though, another explanation for the comparable findings of our DMD sample may be that compensatory mechanisms create alternative networks when dystrophin is absent to at least partly compensate the shortcomings in WM.

\section{Limitations and future perspectives}

This first explorative study assessed whether DMD patients with LD and WM deficiencies benefit from computerized WM training. We determined our sample size based on SCED and for this design a small sample of clinical patients are sufficient to draw conclusions as power is based on the number of repeated measures instead of number of included patients. ${ }^{57}$ We solely included patients aged 10-16 years whilst Jungle Memory $^{\mathrm{TM}}$ is designed for the age range of 7-16 years and as WM grows over time it would be valuable to compare the benefits of training in different ages. Although it is stated that WM training is more beneficial in children aged 10 years or older, because of their insights in their deficits and needs, their increased attention abilities and semantic knowledge, and the more efficient use of memory strategies. ${ }^{9,58}$ Furthermore, our patients were referred to the outpatient clinic CNL on the indication of learning, cognitive or behavioral problems. For this reason, these problems may be more prevalent or severe in our patients than in DMD patients in general, making our results likely less generalizable to the total DMD population. Finally, due to the long 
duration of the study, patients may have changed from class or school between the neurocognitive and behavioural testing measurements. For instance, case 4 changed from primary to secondary school after his assessment at 3 months FU, making his teacher ratings at his latest testing ( 8 months FU) less reliable for comparison with previous outcomes. Additional studies are necessary to confirm and further evaluate near and far transfer effects of computerized WM training and in particular its generalizations to daily life at home and school.

\section{Conclusion}

Computerized WM training may be feasible for DMD patients a comorbid LD with WM problems. It appears to improve WM, academic skills and other cognitive domains for a longer period. However, it remains challenging to exclude influencing effects of motivation, increased self-efficacy, use of strategies and expectancy effects of training on performances. Decreases in WM problems reported by parents were solely found using repeated measures (SCED) suggesting that changes in cognition due to interventions such as computerized training cannot be appropriately measured with single measurement points. For a better understanding of generalization of training effects it would be valuable to repeatedly measure effects to academics and development and to evaluate training effects on brain activity and plasticity with fMRI imaging. 


\section{References}

1. Baddeley A. Working memory: looking back and looking forward. Nat Rev Neurosci 2003;4:829-839.

2. Takeuchi $\mathrm{H}$, Sekiguchi A, Taki $\mathrm{Y}$, et al. Training of working memory impacts structural connectivity. J Neurosci 2010;30:3297-3303.

3. Dahlin E, Neely AS, Larsson A, Bäckman L, Nyberg L. Transfer of learning after updating training mediated by the striatum. Science 2008;320:1510-1512.

4. Eriksson J, Vogel EK, Lansner A, Bergström F, Nyberg L. Neurocognitive architecture of working memory. Neuron 2015;88:33-46.

5. Rapport MD, Orban SA, Kofler MJ, Friedman LM. Do programs designed to train working memory, other executive functions, and attention benefit children with ADHD? A meta-analytic review of cognitive, academic, and behavioral outcomes. Clin Psychol Rev 2013;33:1237-1252.

6. Kremen WS, Jacobsen KC, Xian H, et al. Genetics of verbal working memory processes: a twin study of middle-aged men. Neuropsychology 2007;21:569.

7. Jaeggi SM, BuschkuehI M, Jonides J, Shah P. Short-and long-term benefits of cognitive training. Proc Natl Acad Sci 2011;108:10081-10086.

8. Morrison $A B$, Chein JM. Does working memory training work? The promise and challenges of enhancing cognition by training working memory. Psychon Bull Rev 2011;18:46-60.

9. Peijnenborgh JC, Hurks PM, Aldenkamp AP, Vles JS, Hendriksen JG. Efficacy of working memory training in children and adolescents with learning disabilities: A review study and meta-analysis. Neuropsychol Rehabil 2016;26:645-672.

10. Alloway T. Can interactive working memory training improving learning? Journal of Interactive Learning Research 2012;23:197-207.

11. Alloway TP, Bibile V, Lau G. Computerized working memory training: Can it lead to gains in cognitive skills in students? Computers in Human Behavior 2013;29:632-638.

12. Klingberg T. Training and plasticity of working memory. Trends Cogn Sci 2010;14:317-324.

13. Klingberg T, Fernell E, Olesen PJ, et al. Computerized training of working memory in children with ADHD-a randomized, controlled trial. J Am Acad Child Adolesc Psychiatry 2005;44.

14. Chein JM, Morrison AB. Expanding the mind's workspace: Training and transfer effects with a complex working memory span task. Psychon Bull Rev 2010;17:193-199.

15. Melby-Lervåg $\mathrm{M}$, Hulme $\mathrm{C}$. Is working memory training effective? A meta-analytic review. Dev Psychol 2013;49:270.

16. Bergman-Nutley S, Klingberg T. Effect of working memory training on working memory, arithmetic and following instructions. Psychol Res 2014;78:869-877.

17. Hoffman EP, Brown Jr RH, Kunkel LM. Dystrophin: the protein product of the Duchenne muscular dystrophy locus. Cell 1987;51:919-928.

18. Emery AE. The muscular dystrophies. Lancet 2002;359:687-695.

19. Mercuri E, Muntoni F. Muscular dystrophies. Lancet 2013;381:845-860.

20. Waite A, Brown SC, Blake DJ. The dystrophin-glycoprotein complex in brain development and disease. Trends Neurosci 2012;35:487-496.

21. Hendriksen RG, Hoogland G, Schipper S, Hendriksen JG, Vles JS, Aalbers MW. A possible role of dystrophin in neuronal excitability: a review of the current literature. Neurosci Biobehav Rev 2015;51:255-262.

22. Doorenweerd N, Mahfouz A, van Putten M, et al. Timing and localization of human dystrophin isoform expression provide insights into the cognitive phenotype of Duchenne muscular dystrophy. Sci Rep 2017;7:12575.

23. Lidov $\mathrm{H}$, Byers $\mathrm{T}$, Kunkel L. The distribution of dystrophin in the murine central nervous system: an immunocytochemical study. Neuroscience 1993;54:167-187.

24. Muntoni F, Torelli S, Ferlini A. Dystrophin and mutations: one gene, several proteins, multiple phenotypes. Lancet Neurol 2003;2:731-740.

25. Emery AE, Muntoni F, Quinlivan RC. Duchenne muscular dystrophy: OUP Oxford, 2015. 
26. Rae MG, O'Malley D. Cognitive dysfunction in Duchenne muscular dystrophy: a possible role for neuromodulatory immune molecules. J Neurophysiol 2016;116:1304-1315.

27. Hinton V, De Vivo D, Fee R, Goldstein E, Stern Y. Investigation of poor academic achievement in children with Duchenne muscular dystrophy. Learn Disabil Res Pract 2004;19:146-154.

28. Cyrulnik SE, Fee RJ, Batchelder A, Kiefel J, Goldstein E, Hinton VJ. Cognitive and adaptive deficits in young children with Duchenne muscular dystrophy (DMD). J Int Neuropsychol Soc 2008;14:853-861.

29. Hinton VJ, De Vivo DC, Nereo NE, Goldstein E, Stern Y. Selective deficits in verbal working memory associated with a known genetic etiology: the neuropsychological profile of Duchenne muscular dystrophy. J Int Neuropsychol Soc 2001;7:45-54.

30. Hinton V, De Vivo D, Nereo N, Goldstein E, Stern Y. Poor verbal working memory across intellectual level in boys with Duchenne dystrophy. Neurology 2000;54:2127-2132.

31. Wingeier K, Giger E, Strozzi S, et al. Neuropsychological impairments and the impact of dystrophin mutations on general cognitive functioning of patients with Duchenne muscular dystrophy. J Clin Neurosci 2011;18:90-95.

32. Leaffer EB, Fee RJ, Hinton VJ. Digit span performance in children with dystrophinopathy: a verbal span or working memory contribution? J Int Neuropsychol Soc 2016;22:777-784.

33. Billard C, Gillet P, Barthez MA, Hommet C, Bertrand P. Reading ability and processing in Duchenne muscular dystrophy and spinal muscular atrophy. Dev Med Child Neurol 1998;40:12-20.

34. Mento G, Tarantino V, Bisiacchi PS. The neuropsychological profile of infantile Duchenne muscular dystrophy. Clin Neuropsychol 2011;25:1359-1377.

35. Hendriksen JG, Vles JS. Are males with Duchenne muscular dystrophy at risk for reading disabilities? Pediatr Neurol 2006;34:296-300.

36. Lorusso ML, Civati F, Molteni M, Turconi AC, Bresolin N, D'Angelo MG. Specific profiles of neurocognitive and reading functions in a sample of 42 Italian boys with Duchenne Muscular Dystrophy. Child Neuropsychol 2013;19:350-369.

37. Dunn L, Dunn L. Peabody Picture Vocabulary Test--Dutch Adaptation (PPVT-III-NL)(L. Schlichting, Trans.). Amsterdam: Harcourt Test Publishers, 2005.

38. Wechsler D. WISC-V: Administration and Scoring Manual: NCS Pearson, Incorporated, 2014.

39. Manly T, Anderson V, Crawford J, George, M, , Underbjerg M, Robertson I. TEA-Ch: The test of everyday attention for children manual, Second Edition. 2016.

40. Manly T, Anderson V, Nimmo-Smith I, Turner A, Watson P, Robertson IH. The differential assessment of children's attention: The Test of Everyday Attention for Children (TEA-Ch), normative sample and ADHD performance. J Child Psychol Psychiatr 2001;42:1065-1081.

41. Van den Bos K, Lutje Spelberg H. Continu Benoemen \& Woorden Lezen. Een test voor het diagnosticeren van taal-en leesstoornissen. Handleiding. Amsterdam: Boom Testuitgevers, 2007.

42. de Vos T. TempoTest Automatiseren: Boom test uitgevers, 2010.

43. Huizinga M, Smidts D. BRIEF Executieve Functies Gedragsvragenlijst: Handleiding [Dutch Manual BRIEF Behavior Rating Inventory of Executive Function]. Amsterdam: Hogrefe Uitgevers 2009.

44. Jacobson NS, Truax P. Clinical significance: a statistical approach to defining meaningful change in psychotherapy research. J Consult Clin Psychol 1991;59:12.

45. Campbell TC. An introduction to clinical significance: An alternative index of intervention effect for group experimental designs. Journal of Early Intervention 2005;27:210-227.

46. Tate $R$, Perdices $M$. Single-case experimental designs for clinical research and neurorehabilitation settings: Planning, conduct, analysis and reporting: Routledge, 2019.

47. Parker RI, Vannest KJ, Davis JL. Effect size in single-case research: A review of nine nonoverlap techniques. Behav Modif 2011;35:303-322.

48. Parker RI, Vannest KJ, Davis JL, Sauber SB. Combining nonoverlap and trend for single-case research: Tau-U. Behav Ther 2011;42:284-299.

49. Klingberg $\mathrm{T}$, Forssberg $\mathrm{H}$, Westerberg $\mathrm{H}$. Training of working memory in children with ADHD. J Clin Experimen Neuropsychol 2002;24:781-791.

50. Gray $\mathrm{S}$, Chaban $\mathrm{P}$, Martinussen $\mathrm{R}$, et al. Effects of a computerized working memory training program on working memory, attention, and academics in adolescents with severe LD and comorbid ADHD: a randomized controlled trial. J Child Psychol Psychiatry 2012;53:1277-1284. 
51. Beaudoin $M$, Desrichard $\mathrm{O}$. Are memory self-efficacy and memory performance related? A metaanalysis. Psychol Bull 2011;137:211.

52. Holmes J, Gathercole SE, Dunning DL. Adaptive training leads to sustained enhancement of poor working memory in children. Dev Sci 2009;12:F9-F15.

53. Loomes C, Rasmussen C, Pei J, Manji S, Andrew G. The effect of rehearsal training on working memory span of children with fetal alcohol spectrum disorder. Res Dev Disabil 2008;29:113-124.

54. Apter BJ. Do computerised training programmes designed to improve working memory work? Educ Psychol Pract 2012;28:257-272.

55. Hellebrekers DMJ, Lionarons JM, Faber CG, Klinkenberg S, Vles JSH, Hendriksen JGM. Instruments for the Assessment of Behavioral and Psychosocial Functioning in Duchenne and Becker Muscular Dystrophy; a Systematic Review of the Literature. J Pediatr Psychol 2019.

56. Yoncheva YN, Hardy KK, Lurie DJ, et al. Computerized cognitive training for children with neurofibromatosis type 1: A pilot resting-state fMRI study. Psychiatry Research: Neuroimaging 2017;266:53-58.

57. Kazdin AE. Single-case experimental designs. Evaluating interventions in research and clinical practice. Behav Res Ther 2019;117:3-17.

58. Hendriks M, Dernison R, Vugs B, König C. Geheugen. In H. Swaab, A. Bouma, J. Hendriksen, \& C.König (Eds.). Klinische Kinderneuropsychologie. Amsterdam: Boom, 2011: 163-183. 
<smiles>Cc1ccccc1</smiles> 


\section{Chapter 8}

Methylphenidate use in males with Duchenne

muscular dystrophy and a comorbid

attention-deficit hyperactivity disorder

Judith M. Lionarons, Danique M.J. Hellebrekers", Sylvia Klinkenberg, Faber, C.G., Johan S.H. Vles, Jos G.M. Hendriksen * contributed equally European Journal of Paediatric Neurology 2019;23(1):152-157 


\section{Abstract}

Attention-deficit hyperactivity disorder (ADHD) is a common comorbidity in Duchenne muscular dystrophy (DMD). Until now, treatment with methylphenidate (MPH) has never been systematically assessed and described in this population. Our aim was to evaluate the effectiveness and safety of short acting MPH for learning problems in males with DMD and ADHD. Neuropsychological (cognition and behavior) and medical data of a sample of ten males (mean age=8.1 years, range 6.3-9.8) with DMD and an ADHD diagnosis was retrospectively analyzed at baseline (TO; without MPH), short-term follow-up (T1; with MPH; mean interval T0-T1=8.3 months, range 4.3-15.6), and longterm follow-up (T2; mean interval T1-T2=23.1 months, range 2.6-77.7). An initial MPH dose of $5 \mathrm{mg} /$ day was given on school mornings, with an increase of $2.5-5 \mathrm{mg} /$ week depending on individual tolerance and treatment response, until a sufficiently effective dose was reached (range $0.2-0.6 \mathrm{mg} / \mathrm{kg} /$ day). At $\mathrm{T} 1$, results demonstrated an improvement in attention (i.e. concentration, impulsivity, and distractibility) in four patients. Suboptimal effects were reported in four patients, and no effects in two patients. At T2, seven patients showed considerable improvement in attention. No major side effects were reported. Overall, our data show that short acting MPH can be clinically effective for learning problems in males with DMD and ADHD, with regular cardiac follow-up, and close monitoring of side effects, and neuropsychological effects. Furthermore, this underscores the importance of the use of validated cognitive and behavioral measurements tools with adequate sensitivity to objectively evaluate the effect of MPH. 


\section{Introduction}

Duchenne muscular dystrophy (DMD) is an X-linked neuromuscular disorder with a prevalence of approximately 1 in 5,000 live male births. ${ }^{1}$ It is caused by mutations in the $D M D$ gene $(X p 21)$ that encodes for the dystrophin protein, resulting in the absence of this protein. Absence of dystrophin generally results in characteristic progressive muscle weakness, and eventual fatal cardiac and respiratory complications. ${ }^{2}$ In addition to the progressive muscle weakness, learning, neurocognitive, and behavioral disorders are common in DMD. ${ }^{3-6}$ Particularly, high prevalence rates of attention-deficit hyperactivity disorder (ADHD; 32\% versus $5.3 \%$ in the general population) have been described in $\mathrm{DMD}^{5-7}$, and the milder variant Becker muscular dystrophy, ${ }^{8}$ which often results in learning problems. Consequently, appropriate mental health screening (i.e. Strength and Difficulties Questionnaire (SDQ), and Personal Adjustment and Role Skills scale; PARSIII), neuropsychological assessment (cognition and behavior), and pharmacological treatment with stimulants or $\alpha$-adrenoceptor agonists has been recommended for the diagnosis and treatment of ADHD comorbidity in DMD, as part of the recently updated international standards of care guidelines. ${ }^{9}$ However, the optimum ADHD treatment in DMD is not well characterized, and the effectiveness of stimulants has not been systematically assessed and described in this population. Methylphenidate (MPH) has been shown to reduce ADHD symptoms (i.e. hyperactivity, impulsivity, and inattentive behavior), and improve associated behavior, academics, and social functioning in children, adolescents and adults without DMD. ${ }^{10}$ In DMD, MPH prescription has been limited because of the presence of comorbidities, such as a cardiomyopathy ${ }^{2}$ or epilepsy. ${ }^{11}$ With regard to these comorbidities, sympathomimetic agents (i.e. $\mathrm{MPH}$ ) could potentially provoke complications in these patients. Other potential side effects, such as motor tics, sleep or mood disorders have also been previously reported in neurological disorders. ${ }^{12}$ Therefore, the evaluation of MPH is of great importance especially in this population. The present study reports for the first time a systematic medical and neuropsychological evaluation of MPH treatment in ten patients with DMD and ADHD. Our aim was to evaluate the effectiveness and safety of short acting MPH for learning problems in males with DMD and a comorbid ADHD diagnosis. 


\section{Materials and methods}

\section{Participants}

Reported subjects were males with DMD and a comorbid ADHD diagnosis attending the outpatient clinic of the Kempenhaeghe Centre for Neurological Learning Disabilities, Heeze, The Netherlands. Subjects were included if they met the following inclusion criteria: (1) were males, (2) had a proven mutation of the dystrophin gene, (3) had a diagnosis of ADHD according to the DSM-IV criteria ${ }^{13}$ (4) had medical and neuropsychological data of baseline and short-term follow-up, (5) received no other psychological interventions, except for psychoeducation, on baseline and follow-up, (6) did not use any psychostimulants on T0, and only MPH on T1, and (7) had an adequate proficiency in Dutch. Exclusion criteria were (1) an age younger than three or older than sixteen years at time of inclusion, and (2) physical immobility of upper extremities (hand and arm function), which may affect the neuropsychological test scores. Ethical approval was granted by Kempenhaeghe Ethics Committee and informed parental consent was obtained. The study was conducted in accordance with the 18th World Medical Assembly, Helsinki 194.

\section{Study design}

The diagnosis of ADHD was established by an experienced neuropsychologist (JH) and child neurologist (JV or SK) based on (1) the Diagnostic and Statistical Manual of Mental Disorders-IV criteria for ADHD, ${ }^{13}$ (2) teacher and parents observations, and (3) an extensive neuropsychological assessment. ${ }^{14}$ All subjects received an extensive medical (performed by treating cardiologist and child neurologist JV or SK), and neuropsychological work-up (performed by JH) as part of regular care at baseline (TO). Medical work-up consisted of standard prescribing practices and guidelines with additional care considerations focusing on the general medical condition of each individual subject (i.e. disease status, cardiac status, medication interactions) based on the international standards of care guidelines for DMD. ${ }^{9}$ Cardiac status was evaluated based on standard cardiovascular tract (hetero)anamnesis, blood pressure (BP), heart rate (HR), electrocardiogram (ECG) and non-invasive imaging (e.g. cardiovascular MRI; $\mathrm{CMR}$ ), which were used to establish cardiac function, rhythm abnormalities, and to screen for underlying anatomical abnormalities that could affect cardiovascular health. ${ }^{15}$ Neuropsychological work-up consisted of objective neurocognitive and behavioral assessment, and subjective behavioral observations. Treatment started after parents informed consent, and approval of the treating child cardiologist. All subjects were treated with short acting $\mathrm{MPH}$ on the indication of attention problems which 
were most prominent during school resulting in learning problems. An initial dose of $5 \mathrm{mg} /$ day was given in the morning before school, with an increase of 2.5-5 mg/week depending on individual tolerance, and treatment response, until a sufficiently effective dose was reached (range 0.2-0.6 mg/kg/day). ${ }^{16}$ A second dose for the afternoon school program was given around $12.00 \mathrm{~h}$ in addition to the morning dose if needed based on good clinical practice. None of the subjects used MPH at home, on weekends or holidays. Since behavioral problems were not the main problem in this population, we did not prescribe the extended release MPH over time. Treatment effects and potential adverse effects were evaluated at short-term follow-up (T1), and long-term follow-up (T2) as part of regular care.

\section{Assessments}

For this study, behavioral assessment consisted of subjective behavioral observations from patients, parents, teachers, and the clinical team, and objective assessment using the Child Behavior Checklist-Attention Problems subscale (CBCL-AP). In line with the $\mathrm{CBCL}$ manual, $\mathrm{t}$-scores greater than or equal to 63 were deemed to be in the clinical range. ${ }^{17} \mathrm{~A}$ change in clinical range is defined as a clinically relevant effect. Neurocognitive assessment consisted of the Symbol Search subtest to measure processing speed. Raw scores were converted to standardized age-related scores (SS; with a range of 1-19, mean 10 and standard deviation (SD) of 3 in healthy controls). ${ }^{18}$ In line with the Wechsler manual, a change in SS of 3 is defined as a clinically relevant effect. Full intelligence quotient (FIQ) was assessed by the Wechsler Intelligence Scale for Children-III. ${ }^{18}$ Neuropsychological assessment was focussed on each individual request for help based on good clinical practice, and at least consisted of abovementioned assessment tools.

\section{Statistical analysis}

Descriptive statistics displayed frequencies and means (SD) of demographic and disease-related characteristics. Wilcoxon rank sum tests were used to assess difference between baseline and short-term follow-up of CBCL-AP and processing speed. Underlying assumptions were checked before carrying out actual analyses. Normality distributions of residuals were checked by visual inspection of histograms. All statistical analyses were carried out using SPSS version 24 for MAC OS X. Results were considered statistically significant if $p<.05$. 


\section{Results}

\section{Participants}

Reported subjects were males with DMD and a comorbid ADHD diagnosis (mean age $=8.1, \mathrm{SD}=1.3$, range 6.3-9.8 years). Intelligence quotient (IQ) as assessed by the Wechsler Intelligence Scale for Children- $\mathrm{II}^{18}$ ranged between 66-118 (mean IQ=91.8, $\mathrm{SD}=17.6)$. Subject characteristics are summarized in Table 8.1. Within our clinical sample, subject 7 was previously diagnosed with absence epilepsy; he was seizure free with sodium valproate. Subject 9 had an oppositional defiant disorder, which was treated with dipiperon. Dyslexia was diagnosed in subject 10. Subjects 7-9 had problems with sleeping throughout the night at TO.

Table 8.1 Subject characteristics and MPH effects $(N=10)$.

\begin{tabular}{lllclcc}
\hline Subject & $\begin{array}{l}\text { Age } \\
\text { (years) }\end{array}$ & DNA mutation & $\begin{array}{c}\text { MPH dose T1 } \\
(\mathrm{mg} / \mathrm{kg} / \text { day })\end{array}$ & MPH adverse effect & $\begin{array}{c}\text { T0-T1 } \\
\text { MPH effect }\end{array}$ & $\begin{array}{c}\text { T1- T2 } \\
\text { MPH effect }\end{array}$ \\
\hline 1 & 7 & Deletion exon 8-13 & 0,5 & Delayed sleep onset & + & + \\
2 & 9 & Deletion exon 51-54 & 0,2 & Delayed sleep onset & $+/-$ & - \\
3 & 9 & Deletion exon 49-50 & 0,3 & NAE & + & + \\
4 & 9 & Deletion exon 51 & 0,3 & NAE & + & + \\
5 & 9 & Deletion exon 52 & 0,3 & NAE & + & + \\
6 & 6 & Deletion exon 45 - 50 & 0,2 & Delayed sleep onset & $+/-$ & + \\
7 & 8 & Out of frame exon 45-52 & 0,2 & NAE & $+/-$ & + \\
8 & 7 & Deletion exon 58 & 0,2 & NAE & $+/-$ & + \\
9 & 7 & Deletion exon 46-52 & 0,4 & Delayed sleep onset & - & - \\
10 & 6 & Deletion exon 48-54 & 0,3 & Loss of appetite & - & - \\
\hline
\end{tabular}

mg=milligram, $\mathrm{MPH}=$ methylphenidate, NAE=no adverse effects, T0=neuropsychological baseline measurement, T1=neuropsychological short-term follow-up measurement, T2=long-term regular follow-up, $+=$ subjective reported positive effect of $\mathrm{MPH},+$ /-=subjective reported suboptimal effect $\mathrm{MPH}$, -=subjective reported no effect $\mathrm{MPH}$.

\section{MPH effect at short-term follow-up}

Mean time between T0 and T1 was 8.3 months (SD=3.4, range 4.3-15.6).

\section{Medical effects}

Medical monitoring data concerning BP, HR, height and weight (Body Mass Index) remained within the normal range for the evaluated age category. Regular cardiac follow-up showed no cardiovascular side effects. Additionally, no seizures were noted. Reported side effects consisted of a loss of appetite in subject 10, and delayed sleep onset (subjects $1-2$, subject 6 , subject 9), whereupon melatonin was prescribed in 
subject 1 with success. Of the four patients who reported delayed sleep onset, one patient (subject 1) used a relatively high MPH dosage $(0.5 \mathrm{mg} / \mathrm{kg} /$ day; maximum daily dose of $15 \mathrm{mg}$ in two doses with latest administration time of $12.00 \mathrm{~h}$ ). ${ }^{19}$ One patient (subject 8) with problems sleeping throughout the night at baseline developed delayed sleep onset when using MPH. Two patients discontinued treatment due to a lack of effect (subjects 9-10).

\section{Neuropsychological effects}

At T1, patients, parents, teachers, and the clinical team observed an improvement in attention (i.e. concentration, impulsivity, and distractibility) in four patients (subject 1 , subjects 3-5). Suboptimal effects - which were defined as starting, yet insufficient effects on attention - were observed in four patients (subject 2, subjects 6-8). No effects were noted in subjects 9 and 10 . Behavioral assessments $(n=5)$ showed a trend towards significance ( $\mathrm{Mdn} \mathrm{T0}=63, \mathrm{Mdn} \mathrm{T} 1=57$ ), $\mathrm{z}=-1.841, p=.066$, and a clinically relevant effect (cut off value $<63$ ) in two patients. Neurocognitive assessment $(n=8)$ showed no statistically significant effect ( $\mathrm{Mdn} T 0=11, \mathrm{Mdn} \mathrm{T} 1=12$ ), $\mathrm{z}=-0.736, p=.462$ and in one patient a clinically relevant effect $(S D>1)$ was found.

\section{$\mathrm{MPH}$ effect at long-term follow-up}

Mean time between $\mathrm{T} 1$ and $\mathrm{T} 2$ was 23.1 months ( $\mathrm{SD}=26.7$, range 2.6-77.7). At $\mathrm{T} 2$, Medical monitoring data (i.e. BP and Body Mass Index) were within the normal range and no cardiovascular side effects were described. One patient discontinued treatment due to mood problems (subject 2). Behavioral observations of patients, parents, teachers and the clinical team showed an improvement in attention in seven patients (subject 1 , subjects $3-8$ ). In subjects 6-8 with suboptimal effects at T1 an improvement in attention was observed at $\mathrm{T} 2$.

\section{Discussion}

This is the first study reporting on the clinical effectiveness and safety of short acting MPH treatment for learning problems in ten males with DMD and a comorbid ADHD diagnosis using an extensive medical and neuropsychological work-up at baseline, and short-term follow-up. Results demonstrate that MPH treatment considerably improves attention in seven subjects according to the subjective reports of patients, parents, teachers and the clinical team at long-term follow-up. No major side effects were reported. 


\section{Neuropsychological outcomes}

Literature on neuropsychological evaluation of MPH is scarce in neurological disorders. A recent study evaluated the effect of $\mathrm{MPH}$ in Neurofibromatosis type 1, a neurogenic disorder in which ADHD comorbidity is common as well. Full-scale IQ scores improved significantly in Neurofibromatosis type 1 patients with ADHD who received $\mathrm{MPH}^{20}$ These improvements in full-scale IQ scores were also correlated with an improvement in reaction time variability based on the Test of Variables of Attention. ${ }^{20}$ Furthermore, MPH use has been shown to significantly reduce behavioral and social dysfunction, and causing lower $\mathrm{CBCL}$ scores in Neurofibromatosis type 1 patients with ADHD. ${ }^{21}$ Both the CBCL-AP and the Conners Rating Scale Revised are the most commonly used measurements to support the diagnosis of ADHD in children and adolescents, and previous research on the diagnostic accuracy of these scales yielded moderate sensitivity and specificity in supporting the diagnosis of ADHD. ${ }^{22}$ Unfortunately, in our study only five patients completed the CBCL-AP at baseline and short-term follow-up, thus our results are rather explorative.

\section{Side effects}

Reported side effects in our subjects were having difficulty falling asleep, which is in line with previous finings in patients with ADHD without comorbidity. ${ }^{12}$ Stimulants may exacerbate delayed sleep-onset, but may also be related to a rebound effect - increase over baseline values in ADHD symptoms when MPH wears off - rather than to the medication itself. ${ }^{23}$ Notably, difficulty falling asleep often occurs during titration, and may improve over time. ${ }^{24}$ Management of sleep problems in our subjects consisted of sleep problems and health education, and melatonin prescription with positive results, which is in line with previous research. ${ }^{25,}{ }^{26}$ Neurological side effects, such as motor tics or seizures, were not found in our subjects, even though one patient had epilepsy. MPH has also a potential impact on cardiac functioning which may have clinical consequences, especially in DMD patients. It is a sympathomimetic agent that increases noradrenergic and dopaminergic transmission, which affects heart HR and BP. ${ }^{27} \mathrm{~A}$ recent systematic review on cardiovascular effects of $\mathrm{MPH}$ in children and adolescents with ADHD found a significant effect on systolic BP. Since this is considered a risk factor for cardiovascular morbidity and mortality during adult life, it was recommended that BP and HR should be monitored closely and regularly. ${ }^{28}$ As cardiac management is already part of regular care of DMD patients, all subjects were regularly seen and monitored by their child cardiologist, and BP and HR remained stable from baseline to follow-up. 


\section{Limitations}

Due to our small sample, there might be a lack of power to observe effects on the objective neuropsychological outcome variables. Additionally, the time between the baseline and short-term follow-up was not equal for each subject, and may have been too short to measure clinical effects on cognition. The ten subjects which are described in this study, where all seen for regular outpatient clinical care. Information of certain cognitive variables (working memory and attention measures) and behavioral variables (CBCL-AP) were limited or not available. Thereby the change between baseline and short-term follow-up on these measures could not be analyzed accurately.

\section{Future perspectives}

Further prospective research should include a follow-up time of at least six months and one year, and needs to include an age-matched control group of DMD males with a comorbid ADHD, without MPH or receiving other treatment to determine whether the effects are caused by MPH treatment. To further evaluate the different dose effects of $\mathrm{MPH}$ in this population, a second long-term follow-up neuropsychological work-up should be included. This neuropsychological work-up should be performed using a standard protocol of validated ADHD specific tools, such as the CBCL-AP or Conners Parent Rating Scale ${ }^{22} /$ IOWA Conners Rating Scale ${ }^{29}$, as well as the SDQ and PARSIII as recommended in the international standards of care guidelines for DMD. ${ }^{9}$ Importantly, since these measurement tools are developed for the general population, certain items involving physical mobility may not be applicable for patients with impaired motor function, and should be interpreted with caution. Whether these ADHD specific tools are sensitive for ADHD comorbidity in DMD patients should be further investigated. Eventually, the effect of MPH should be evaluated in a larger sample preferably using a randomized control trial design.

\section{Conclusion}

Current data shows clinically effective use of short acting MPH for learning problems in males with DMD and a comorbid ADHD diagnosis, with regular cardiac follow-up, and close monitoring of side effects and neuropsychological effects. Overall, our results underscore the importance of the use of validated behavioral - psychosocial measurements tools, and use of psychopharmacological interventions in DMD as recommended by the international standards of care guidelines for DMD. 


\section{References}

1. Moat SJ, Bradley DM, Salmon R, Clarke A, Hartley L. Newborn bloodspot screening for Duchenne muscular dystrophy: 21 years experience in Wales (UK). Eur J Hum Genet 2013;21:1049-1053.

2. Yiu EM, Kornberg AJ. Duchenne muscular dystrophy. J Paediatr Child Health 2015;51:759-764.

3. Hendriksen JG, Vles JS. Neuropsychiatric disorders in males with duchenne muscular dystrophy: frequency rate of attention-deficit hyperactivity disorder (ADHD), autism spectrum disorder, and obsessive--compulsive disorder. J Child Neurol 2008;23:477-481.

4. Snow WM, Anderson JE, Jakobson LS. Neuropsychological and neurobehavioral functioning in Duchenne muscular dystrophy: a review. Neurosci Biobehav Rev 2013;37:743-752.

5. Pane $M$, Lombardo ME, Alfieri $P$, et al. Attention deficit hyperactivity disorder and cognitive function in Duchenne muscular dystrophy: phenotype-genotype correlation. J Pediatr 2012;161:705-709 e701.

6. Anderson JL, Head SI, Rae C, Morley JW. Brain function in Duchenne muscular dystrophy. Brain 2002;125:4-13.

7. Polanczyk G, de Lima MS, Horta BL, Biederman J, Rohde LA. The worldwide prevalence of ADHD: a systematic review and metaregression analysis. Am J Psychiatry 2007;164:942-948.

8. Young HK, Barton BA, Waisbren S, et al. Cognitive and psychological profile of males with Becker muscular dystrophy. J Child Neurol 2008;23:155-162.

9. Birnkrant DJ, Bushby K, Bann CM, et al. Diagnosis and management of Duchenne muscular dystrophy, part 3: primary care, emergency management, psychosocial care, and transitions of care across the lifespan. Lancet Neurol 2018;17:445-455.

10. Greenhill LL, Pliszka S, Dulcan MK, et al. Summary of the practice parameter for the use of stimulant medications in the treatment of children, adolescents, and adults. J Am Acad Child Adolesc Psychiatry 2001;40:1352-1355.

11. Hendriksen RGF, Vles JSH, Aalbers MW, Chin RFM, Hendriksen JGM. Brain-related comorbidities in boys and men with Duchenne Muscular Dystrophy: A descriptive study. Eur J Paediatr Neurol 2018;22: 488-497.

12. Groenman AP, Schweren L, Dietrich A, Hoekstra PJ. An update on the safety of psychostimulants for the treatment of attention-deficit/hyperactivity disorder. Expert Opin Drug Saf 2017;16:455-464.

13. Frances A, Pincus, HA, First, MB. Diagnostic and statistical manual of mental disorders: DSM-IV: Washington DC: American Psychiatric Association, 1994.

14. Hendriksen JG, Peijnenborgh JC, Aldenkamp AP, Vles JS. Diagnostic overshadowing in a population of children with neurological disabilities: A cross sectional descriptive study on acquired ADHD. Eur J Paediatr Neurol 2015;19:521-524.

15. Birnkrant DJ, Bushby K, Bann CM, et al. Diagnosis and management of Duchenne muscular dystrophy, part 2: respiratory, cardiac, bone health, and orthopaedic management. Lancet Neurol 2018;17: 347-361.

16. Huss M, Duhan P, Gandhi P, Chen CW, Spannhuth C, Kumar V. Methylphenidate dose optimization for ADHD treatment: review of safety, efficacy, and clinical necessity. Neuropsychiatr Dis Treat 2017;13:1741-1751.

17. Achenbach TM, Edelbrock CS. Manual for the child behavior checklist and revised child behavior profile. 1983.

18. Wechsler D. Manual for the Wechsler intelligence scale for children-(WISC-III). San Antonio, TX: The Psychological Corporation 1991.

19. Konrad K, Gunther T, Hanisch C, Herpertz-Dahlmann B. Differential effects of methylphenidate on attentional functions in children with attention-deficit/hyperactivity disorder. J Am Acad Child Adolesc Psychiatry 2004;43:191-198.

20. Lidzba K, Granstroem S, Leark RA, Kraegeloh-Mann I, Mautner VF. Pharmacotherapy of attention deficit in neurofibromatosis type 1: effects on cognition. Neuropediatrics 2014;45:240-246.

21. Mautner VF, Kluwe L, Thakker SD, Leark RA. Treatment of ADHD in neurofibromatosis type 1. Dev Med Child Neurol 2002;44:164-170. 
22. Chang L-Y, Wang M-Y, Tsai P-S. Diagnostic Accuracy of Rating Scales for Attention-Deficit/Hyperactivity Disorder: A Meta-analysis. Pediatrics 2016;137:e20152749.

23. Lecendreux M, Cortese S. Sleep problems associated with ADHD: a review of current therapeutic options and recommendations for the future. Expert Rev Neurother 2007;7:1799-1806.

24. Stein MA, Weiss M, Hlavaty L. ADHD treatments, sleep, and sleep problems: complex associations. Neurotherapeutics 2012;9:509-517.

25. Tjon Pian Gi CV, Broeren JPA, Starreveld JS, FG AV. Melatonin for treatment of sleeping disorders in children with attention deficit/hyperactivity disorder: a preliminary open label study. Eur J Pediatr 2003;162:554-555.

26. Van der Heijden KB, Smits MG, Van Someren EJ, Ridderinkhof KR, Gunning WB. Effect of melatonin on sleep, behavior, and cognition in ADHD and chronic sleep-onset insomnia. J Am Acad Child Adolesc Psychiatry 2007; 46:233-241.

27. Volkow ND, Wang GJ, Fowler JS, et al. Cardiovascular effects of methylphenidate in humans are associated with increases of dopamine in brain and of epinephrine in plasma. Psychopharmacology (Berl) 2003;166:264-270.

28. Hennissen L, Bakker MJ, Banaschewski T, et al. Cardiovascular Effects of Stimulant and Non-Stimulant Medication for Children and Adolescents with ADHD: A Systematic Review and Meta-Analysis of Trials of Methylphenidate, Amphetamines and Atomoxetine. CNS Drugs 2017;31:199-215.

29. Waschbusch DA, Willoughby MT. Parent and teacher ratings on the IOWA Conners Rating Scale. Journal of Psychopathology and Behavioral Assessment 2008;30:180. 
<smiles>Cc1ccccc1</smiles> 


\section{Chapter 9}

General discussion 
Chapter 9 
The main aim of this thesis was to provide new insights on neurocognition and behaviour, diagnostic work up and interventions in Duchenne (DMD) and Becker muscular dystrophy (BMD). In this chapter the main findings of the studies are reported and implications of the results for clinical practice and scientific research are discussed.

\section{A summary of main findings}

- Gene mutations affecting the production of dystrophin protein isoforms may not only predict the neurocognitive and behavioural features in paediatric DMD (Chapter 2) and BMD patients (Chapter 5).

- In DMD, a developmental stagnation in verbal span capacity may exist and appears to be more pronounced in patients missing Dp140 isoform (Chapter 3).

- Neurocognitive and behavioural features are less prominent in adult BMD patients and no relevant correlations are found with disease severity (Chapter 4).

- In DMD, a wide variety of assessment instruments for behavioural and psychosocial functioning have been used. Analyses of psychometric properties and applicability of instruments revealed that three instruments are potentially valid for psychosocial screening: the Psychosocial Adjustment and Role Skills Scale $3^{\text {rd }}$ edition (PARS-III), the Life Satisfaction Inventory for Adolescents with DMD and the Paediatric Quality of Life Inventory Generic Module (PedsQL GM) (Chapter 6).

- Promising short and long-term results of computerized WM training are shown in a small sample of patients with DMD $(\mathrm{N}=4)$ and a comorbid learning disability with working memory deficiencies using Single Case Experimental Design (SCED; Chapter 7).

- Preliminary results showed that short acting stimulant medication (methylphenidate) may be clinically effective in patients with DMD and an attention-deficit hyperactivity disorder $(\mathrm{N}=10)$. No side effects were reported (Chapter 8).

\section{Discussion of the results}

Duchenne and Becker muscular dystrophy are neuromuscular disorders in which brainrelated comorbidities in neurocognition and behaviour are described. ${ }^{1-9}$ The origin of these comorbidities have been linked to the role of dystrophin deficiencies in the brain and it interaction with other proteins of the dystrophin glycoprotein complexes (e.g. syntrophin). ${ }^{3,10-16}$ It is questionable whether brain dystrophin determines the presence 
as well as the severity of these brain-related comorbidities when considering the main findings of present thesis. In chapter $\mathbf{2}$ we compared DMD males to age matched males with another multifactorial, neurogenetic disorder, Neurofibromatosis type 1 . We found no significant differences between these two groups on cognitive phenotypes i.e. in full-scale intellectual functioning (FSIQ), verbal short-term memory/span capacity, sustained and visual attention, processing speed and visuospatial abilities. Additionally, behavioural reports of parents and teachers of the two groups showed no significant differences. In chapter $\mathbf{5}$, we evaluated the cognitive phenotypes of three brothers with BMD who had a similar dystrophin gene mutation (involving exon 45-48), were tested at a similar age and grow-up in a similar environment. The cognitive phenotypes of these brothers differed in several cognitive domains i.e. in FSIQ, verbal short-term memory/span capacity, attention and reading. Their cognitive development differed as well. The brothers had only similar difficulties in processing speed and math. In chapter 4 we also noticed no obvious neurocognitive or behavioural dystrophinopathy profile in adult males with BMD. Despite the fact that the adult BMD males exhibited lowaverage intellectual properties with minor deficits in executive functions, there outcomes fell within normal range. Though it is important to keep in mind that in both BMD studies (chapter 4 and 5) no information was available on whether specific brain dystrophin isoform expression (i.e. Dp140) is disturbed. This makes it difficult to predict whether a loss of Dp140 in addition to the full-length dystrophin isoform (Dp427 ) may have resulted in more specific or severe cognitive and behavioural problems in both studies. For instance in our DMD sample in chapter $\mathbf{3}$ we found that the additional loss of Dp140 was linked to more problems in verbal short-term memory/span capacity compared to DMD patients with intact Dp140 expression. Nevertheless, the proposed mechanisms involved in neurocognitive and behavioural functioning remain versatile ${ }^{16}$ and questions may arise on the role of (specific) brain dystrophin expression in relation to the phenotypes of DMD and BMD patients. In chapter 7 and chapter 8 we for instance found that interventions such as computerized working memory training (chapter 7) and methylphenidate treatment (chapter 8) in DMD patients with comorbidities in cognition, behaviour or learning are feasible, safe and have a similar (positive) effect in our DMD patient samples compared to other patient populations. It was expected that these treatments had no comparable positive effect due to the absence of dystrophin in brain areas and networks responsible for these neurocognitive and behavioural functions. Nevertheless, the findings of present thesis acknowledge that in DMD and BMD, problems may arise in neurocognition and behaviour. Our results are in line with previous studies and reveal that FSIQ is approximately one standard deviation lower (mean IQ 85) in DMD and approximately normal in BMD (mean estimated IQ 91) compared to norm population (chapter 2, 4 and 5). ${ }^{5-7,17,18}$ 
Additionally, we found processing speed difficulties in both our DMD and BMD samples (chapter 3, 4 and 5) despite the exclusion of patients with impaired functions of upper extremities. We further confirmed the consistent cognitive feature i.e. limited verbal short-term memory/verbal span capacity in DMD (chapter 2, 3 and 7). 4,14,19-23 It appears that BMD patients may exhibit some problems in this function as well (chapter 4). Verbal short-term memory and span capacity are important and powerful predictors for academic skills such as reading, writing and math. ${ }^{19,22-25}$ Therefore, it is important that possible deficits are indicated at an early age, in particular because these verbal memory / span deficits may remain present longitudinally (chapter $\mathbf{3}$ ). This reflects that the suggested (re) evaluation of cognition, behaviour and learning and it development is of great importance, especially during transitioning phases from for instance childhood to adolescence. ${ }^{26}$ Previous studies used various methods to evaluate the brain-related comorbidities making it difficult to characterize specific profiles and to compare outcomes of studies. For instance in chapter 6 we found that 61 behavioural and psychosocial instruments (e.g. questionnaires and interviews) were used in 51 previous DMD and 3 BMD studies. An essential step for clinicians and researchers is to determine whether it is necessary to develop DMD and BMD specific testing batteries for the evaluation of cognitive and behavioural functioning. It seems that the deficits in FSIQ and verbal short-term memory/span capacity and learning disabilities in reading are found regardless of instruments used in DMD.,14,17,19-22,24,27-29 Though, results on attention, planning, speed and visuospatial deficits differ between previous studies. ${ }^{4,14,21,30,31}$ This may depend on the different cognitive tasks used by previous studies. However, it also likely that these deficits are not consistent in DMD. With regard to behaviour, it was noticed that the prevalence rates of disorders vary between DMD studies. Some display high rates of for instance ADHD symptoms (32\%) whereas others found no symptoms (0\%). ${ }^{32,33}$ In our thesis, chapter $\mathbf{2}$, we also found that some DMD patients had a diagnosis of ADHD, but not all scored in the clinical range for attention problems using the Child Behaviour Checklist (CBCL). This finding in addition to our results of chapter 6 emphasize that certain behavioural (screening) instruments may have underidentified behavioural symptoms or disorders in previous studies. Due to the overlap in symptoms clinicians and researchers should never diagnose conditions based on the results of one screening instruments. For definite diagnostics a multimethod-, source- setting is the most appropriate approach for assessment.

Overall, it remains difficult to attribute the brain-related comorbidities i.e. the neurocognitive impairments and behavioural disorders of DMD and BMD patients solely to the aberrant expression of (specific) brain dystrophin isoforms. So far no single mechanism can be identified for the brain-related comorbidities. Previous DMD 
neuroimaging studies also found no single brain (structure) abnormality in patients,. They rather found individual variability in brain structures, networks, perfusion and metabolism. ${ }^{12}$ Future DMD and BMD studies should determine which mechanisms play a role in the presence and the differentiation of minor to severe problems in neurocognition and behaviour. This would provide better diagnostic work-up and would facilitate the use of targeted therapeutic interventions.

\section{What does this thesis contribute?}

\section{Implications for clinical practice}

- (Re) evaluation of neurocognition and behaviour is recommended in DMD and BMD because one or more impairments and disorders may occur.

- Implementing a diagnostic battery for (re) evaluation of neurocognitive impairments to characterize the incidence and severity of deficits in both patient populations.

- Implementing a gold-standard protocol for screening and diagnosing behavioural, emotional and psychosocial problems in both patient populations.

- Training of cognition in particular working memory may be an effective intervention in patients with DMD and a comorbid learning disability.

- Stimulant medication may be a safe and effective treatment for ADHD symptoms in patients with $\mathrm{DMD}$, but close and regular evaluation of side effects is important.

\section{Implications for future research}

- Connecting neurocognitive and behavioural outcomes to genetics, neurophysiological alterations and neuroimaging to better determine the factors involved in the presence and severity of the DMD and BMD cognitive and behavioural phenotypes.

- Longitudinal follow up of neurocognitive, behavioural and academic assessment to investigate whether $\mathrm{DMD}$ or $\mathrm{BMD}$ patients further grow in or out their comorbidities.

- Additional evaluation of psychometrics of psychosocial screening questionnaires; PARS-III, the LSIA, the PedsQL GM, and the behavioural screening questionnaires; the SDQ, GAD-7 and PHQ-9 for DMD and BMD.

- Developing a gold-standard protocol for screening and diagnosing behavioural and psychosocial problems in DMD and BMD. 
- Evaluating possible (long-lasting) neuronal effects of cognitive interventions in DMD and BMD using $\mathrm{FMRI}$ imaging.

- Using SCED methodology to evaluate intervention effects in a small DMD or BMD patient samples.

- Evaluating the effectivity and safety of psychopharmacological medication (e.g. MPH for ADHD comorbidity) in larger sample sizes preferably using a randomized control trial design or SCED and with additional neurocognitive and behavioural outcomes 


\section{References}

1. Snow WM, Anderson JE, Jakobson LS. Neuropsychological and neurobehavioral functioning in Duchenne muscular dystrophy: a review. Neurosci Biobehav Rev 2013;37:743-752.

2. Ricotti V, Mandy WP, Scoto M, et al. Neurodevelopmental, emotional, and behavioural problems in Duchenne muscular dystrophy in relation to underlying dystrophin gene mutations. Dev Med Child Neurol 2016;58:77-84.

3. Chamova T, Guergueltcheva V, Raycheva M, et al. Association between loss of dp140 and cognitive impairment in duchenne and becker dystrophies. Balkan J Med Genet 2013;16:21-29.

4. Battini R, Chieffo D, Bulgheroni S, et al. Cognitive profile in Duchenne muscular dystrophy boys without intellectual disability: The role of executive functions. Neuromuscul Disord. 2018;28(2):122-128.

5. Young HK, Barton BA, Waisbren S, et al. Cognitive and psychological profile of males with becker muscular dystrophy. J Child Neurol 2008;23:155-162.

6. Lambert JT, Darmahkasih AJ, Horn PS, et al. Neurodevelopmental, Behavioral, and Emotional Symptoms in Becker Muscular Dystrophy. Muscle Nerve. 2020;61(2):156-162.

7. Mori-Yoshimura M, Mizuno Y, Yoshida S, et al. Psychiatric and neurodevelopmental aspects of Becker muscular dystrophy. Neuromuscul Disord 2019;29(12):930-939.

8. Bardoni A, Felisari G, Sironi M, et al. Loss of Dp140 regulatory sequences is associated with cognitive impairment in dystrophinopathies. Neuromuscul Disord 2000;10:194-199.

9. Hendriksen RG, Vles JS, Aalbers MW, Chin RF, Hendriksen JG. Brain-related comorbidities in boys and men with duchenne muscular dystrophy: a descriptive study. Eur J Paediatr Neurol. 2018;22(3):488-497.

10. Waite A, Brown SC, Blake DJ. The dystrophin-glycoprotein complex in brain development and disease. Trends Neurosci 2012;35:487-496.

11. Hendriksen RG, Hoogland G, Schipper S, Hendriksen JG, Vles JS, Aalbers MW. A possible role of dystrophin in neuronal excitability: a review of the current literature. Neurosci Biobehav Rev 2015;51: 255-262.

12. Doorenweerd N. Combining genetics, neuropsychology and neuroimaging to improve understanding of brain involvement in Duchenne muscular dystrophy-a narrative review. Neuromuscul Disord 2020; 30(6):437-442.

13. Doorenweerd N, Mahfouz A, van Putten M, et al. Timing and localization of human dystrophin isoform expression provide insights into the cognitive phenotype of Duchenne muscular dystrophy. Sci Rep 2017;7:12575.

14. Wingeier K, Giger E, Strozzi S, et al. Neuropsychological impairments and the impact of dystrophin mutations on general cognitive functioning of patients with Duchenne muscular dystrophy. J Clin Neurosci 2011;18:90-95.

15. Thangarajh M, Hendriksen J, McDermott M, Martens W, Hart K, Griggs R. Relationships between DMD mutations and neurodevelopment in dystrophinopathy (P5. 4-002). AAN Enterprises, 2019.

16. Rae MG, O'Malley D. Cognitive dysfunction in Duchenne muscular dystrophy: a possible role for neuromodulatory immune molecules. J Neurophysiol 2016;116:1304-1315.

17. Cotton S, Voudouris NJ, Greenwood KM. Intelligence and Duchenne muscular dystrophy: Full-Scale, Verbal, and Performance intelligence quotients. Dev Med Child Neurol 2001;43:497-501.

18. Cotton SM, Voudouris NJ, Greenwood KM. Association between intellectual functioning and age in children and young adults with Duchenne muscular dystrophy: further results from a meta-analysis. Dev Med Child Neurol 2005;47:257-265.

19. Hinton V, Fee R, Goldstein E, De Vivo D. Verbal and memory skills in males with Duchenne muscular dystrophy. Dev Med Child Neurol 2007;49:123-128.

20. Wicksell RK, Kihlgren M, Melin L, Eeg-Olofsson O. Specific cognitive deficits are common in children with Duchenne muscular dystrophy. Dev Med Child Neurol 2004;46:154-159.

21. Cyrulnik SE, Fee RJ, Batchelder A, Kiefel J, Goldstein E, Hinton VJ. Cognitive and adaptive deficits in young children with Duchenne muscular dystrophy (DMD). J Int Neuropsychol Soc 2008;14:853-861.

22. Leaffer EB, Fee RJ, Hinton VJ. Digit span performance in children with dystrophinopathy: a verbal span or working memory contribution? J Int Neuropsychol Soc 2016;22:777-784. 
23. D'Angelo MG, Lorusso ML, Civati F, et al. Neurocognitive profiles in Duchenne muscular dystrophy and gene mutation site. Pediatr Neurol 2011;45:292-299.

24. Billard C, Gillet $P$, Barthez MA, Hommet $C$, Bertrand $P$. Reading ability and processing in Duchenne muscular dystrophy and spinal muscular atrophy. Dev Med Child Neurol 1998;40:12-20.

25. Alloway TP, Alloway RG. Investigating the predictive roles of working memory and IQ in academic attainment. Journal of experimental child psychology 2010;106:20-29.

26. Birnkrant DJ, Bushby K, Bann CM, et al. Diagnosis and management of Duchenne muscular dystrophy, part 3: primary care, emergency management, psychosocial care, and transitions of care across the lifespan. Lancet Neurol 2018;17(5):445-455.

27. Hinton V, De Vivo D, Nereo N, Goldstein E, Stern Y. Poor verbal working memory across intellectual level in boys with Duchenne dystrophy. Neurology 2000;54:2127-2132.

28. Bresolin N, Castelli E, Comi GP, et al. Cognitive impairment in Duchenne muscular dystrophy. Neuromuscular disorders : NMD 1994;4:359-369.

29. Lorusso ML, Civati F, Molteni M, Turconi AC, Bresolin N, D'Angelo MG. Specific profiles of neurocognitive and reading functions in a sample of 42 Italian boys with Duchenne Muscular Dystrophy. Child Neuropsychol 2013;19:350-369.

30. Billard C, Gillet P, Signoret J, et al. Cognitive functions in Duchenne muscular dystrophy: a reappraisal and comparison with spinal muscular atrophy. J Neuromuscul Dis 1992;2:371-378.

31. Hellebrekers DM, Doorenweerd N, Sweere DJ, et al. Longitudinal follow-up of verbal span and processing speed in Duchenne muscular dystrophy. Eur J Paediatr Neurol 2020;25: 120-126.

32. Colombo $P$, Nobile $M$, Tesei $A$, et al. Assessing mental health in boys with Duchenne muscular dystrophy: Emotional, behavioural and neurodevelopmental profile in an Italian clinical sample. Eur J Paediatr Neurol 2017;21(4):639-647.

33. Pane $M$, Lombardo $M E$, Alfieri $P$, et al. Attention deficit hyperactivity disorder and cognitive function in Duchenne muscular dystrophy: phenotype-genotype correlation. J Pediatr 2012;161:705-709. e701. 
<smiles>Cc1ccccc1</smiles> 


\section{Addendum}

Summary

Samenvatting

Valorisation

Dankwoord

Curriculum vitae

Publications and presentations 
Addendum 


\section{Summary}

In Chapter 1, we introduced the subject and the aims of this thesis.

Duchenne (DMD) and Becker (BMD) muscular dystrophy are heritable progressive neuromuscular disorders that are caused by mutations in the dystrophin (DMD) gene. Both disorders predominantly occur in boys. Mutations in the DMD gene result in an absence of the dystrophin protein in DMD and a partial production of dystrophin in the less severe variant BMD.

The $D M D$ gene is responsible for encoding multiple dystrophin isoforms in various tissues (i.e. muscles, kidneys, retina and the brain). Previous research has focused on the relation between disturbed brain dystrophin expression and the presence and severity of brain-related comorbidities such as neurocognitive and behavioural problems. For both disorders, additional information on the existence of dystrophinassociated neurocognitive and behavioural problems is recommended. Furthermore, with regard to the diagnostic work-up, various measurements have been used to evaluate the presence of behavioural and psychosocial problems in DMD and BMD, but an overview of adequate measurements is lacking. Finally, interventions that may limit the influence of the brain-related comorbidities have not been evaluated scientifically previously. In this thesis, we aimed to examine the (1) presence of dystrophinassociated neurocognitive and behavioural problems in DMD and BMD, (2) diagnostic work-up for the assessment of behavioural and psychosocial problems in DMD and BMD and (3) treatment effects i.e. a psychological intervention (i.e. cognitive working memory training) and a psychopharmaceutic (methylphenidate) treatment in DMD.

In Chapter 2, we compared the presence of disorder specific differences in neurocognition and behaviour of male patients with DMD to male patients with Neurofibromatosis type 1 (NF1). NF1 is caused by mutations in the NF1 gene which is responsible for the production of the neurofibromin protein. This protein is localized in various tissues such as in neurons and glial cells in the brain. Previous NF1 literature has focused on a relation between the disturb production of neurofibromin in brain tissue and the presence of cognitive and learning problems. In current study, patients of the DMD and NF1 groups underwent neurocognitive assessment for regular clinical care. Results displayed that males with DMD not significantly differ in intellectual abilities, verbal (working) memory, visuospatial abilities and sustained attention compared to males with NF1. Results of behavioural questionnaires completed by parents and teachers displayed no significant differences between the DMD and NF1 group. On average, overlapping neurocognitive and behavioural problems were found for the 
neurogenetic disorders, DMD and NF1, suggesting that these problems are not solely caused by gene mutations resulting in a lack of expression of one specific protein.

In Chapter 3, we longitudinally assessed neurocognitive data on verbal span capacity and information processing speed of males with DMD. Additionally, we explored the presence of a genotype-phenotype relation. The outcomes of span capacity and processing speed of the total sample were compared at two time points. The cohort was further dived in subgroups to explore associations between the dystrophin brain isoform (Dp140) and performances on verbal span and processing speed. For the total group we found lower verbal span capacity at the first and second assessment, whereas processing speed was normal at both assessments. Subgroup analyses suggested lower scores on verbal span and processing speed for males missing the brain dystrophin isoform, Dp140, compared to males with intact Dp140 expression. In DMD, a developmental stagnation in verbal span capacity, irrespective of processing speed, is detected through longitudinal follow-up. This stagnation appears to be more pronounced in males missing the brain dystrophin isoform, Dp140.

In Chapter 4, outcomes of neurocognitive testing and self-report behavioural questionnaires were used to described the neurocognitive and behavioural features of adult BMD patients. Results of the BMD group were compared to norm data and were additionally correlated with disease severity measures (i.e. motor function tests such as ten meter run/walk test and the Performance Upper limb test 1.2). Results showed that the (estimated) intellectual abilities, verbal memory, processing speed and executive functions were below average compared to the norm population, though outcomes still fell within normal clinical ranges. Results of self-report questionnaires were on average normal. Our findings reveal that adult BMD patients may exhibit minor neurocognitive impairments and no significant behavioural problems.

In Chapter 5, we described the neurocognitive and behavioural profiles of three brothers with BMD carrying a similar in-frame deletion of exons 45-48. The intellectual abilities of the brothers ranged from normal to high. All had difficulties with processing speed and math. Outcomes of executive functioning, working memory, attention and reading differed between the brothers. In addition, a variability in cognitive development between the brothers was noted as well. Our findings suggest that the cognitive and behavioural features of paediatric BMD patients may vary regardless of gene mutations and exposer to similar environmental factors. 
In Chapter 6, we described a systematic review on instruments used to assess behavioural and psychosocial functioning of patients with DMD and BMD. Additionally, we reviewed the psychometric properties and applicability of the instruments used. Sixty-one instruments were used in 54 studies (51 DMD and 3 BMD) of which the CBCL is the most commonly used instrument, despite that it lacks disease specific psychometric information. Based on our psychometric results, three instruments can be appropriate for screening of psychosocial problems: the Psychosocial Adjustment and Role Skills Scale $3^{\text {rd }}$ edition, the Paediatric Quality of Life Inventory Generic module, and the Life Satisfaction Index for Adolescents with Duchenne muscular dystrophy. Psychometric properties of screening instruments for behavioural and emotional problems should be further evaluated, though appropriate instruments may be: the Strengths and Difficulties Questionnaire, the Generalized Anxiety Disorder-7 item questionnaire, and the Patient Health Questionnaire. To ascertain a gold standard for screening of behavioural, emotional and psychosocial functioning of DMD and BMD patients further research should evaluate the abovementioned instruments. For definite diagnostics of behavioural disorders, it is recommended to administer the gold standard multi-method, multi-source and multi-setting assessment, because of the high risk for overlapping comorbidities in DMD and BMD.

In Chapter 7, we studied whether (1) computerized training improves dystrophinassociated verbal working memory problems of DMD patients, (2) effects of training are present immediately at post-intervention, at 3 and 8 months follow-up and (3) training improves problems that arise from the learning disabilities of the patients. We found short and long-term, near and far transfer effects for verbal and visual working memory, academics (reading and math), processing speed and fluid reasoning. Parent questionnaires showed minimal changes in executive behaviour, whereas teachers reported no changes at all time-points. Though, the repeated measures of parent reports on working memory functioning displayed positive effects of training. These effects were evaluated using single case experimental design analyses. Our findings show promising results of computerized working memory training in DMD patients with a comorbid learning disability and working memory problems. Our explorative near and far transfer effects are comparable to outcomes of previous studies evaluating patient populations with learning disabilities or ADHD without DMD. We might speculate that dystrophin is not involved in working memory networks as previously expected.

In Chapter 8, we evaluated the effectiveness and safety of methylphenidate (MPH) treatment for learning problems in males with DMD and comorbid attention-deficit hyperactivity disorder (ADHD). At short-term follow-up an improvement in attention 
(i.e. concentration, impulsivity and distractibility) was noticed in four of ten patients. Suboptimal effects were reported in another four patients and no effects were found for two patients. At long-term follow-up, seven patients considerably improved in attention. No major medical side effects were reported. Our data showed that MPH can be clinically effective for learning problems in DMD with comorbid ADHD. Regular cardiac follow-up and close monitoring of it side effects and neuropsychological effects is recommended.

Overall, the studies in this thesis provide new information on brain-related comorbidities in cognition and behaviour in DMD and BMD. Evaluation of cognition and behavioural functions and their development is of great importance for both neuromuscular disorders. No consensus is shown on diagnostic work-up of behavioural and psychosocial problems in DMD and BMD. Additionally, we presented first positive effects of psychological (i.e. cognitive) interventions and psychopharmacological treatment to reduce brain-related comorbidities in DMD. Future studies should elucidate the mechanisms (e.g. genetics, neurophysiological alterations and brain structure abnormalities) that may underlie the brain-related comorbidities and how these influence the severity of the comorbidities. This would improve the diagnostic work-up and would facilitate the use of appropriate and targeted interventions. 


\section{Samenvatting}

In hoofdstuk 1 introduceren we het onderwerp en het doel van dit proefschrift.

Duchenne (DMD) en Becker (BMD) Spierdystrofie, zijn erfelijke, progressieve spierziekten die veroorzaakt worden door veranderingen oftewel mutaties in het Duchenne Muscular Dystrophy $(D M D)$ gen. Dit gen is onderdeel van het erfelijk materiaal, het DNA. Deze spierziekten komen vrijwel uitsluitend voor bij jongens.

De mutaties in het DMD-gen zorgen ervoor dat het eiwit dystrofine niet - bij Duchenne Spierdystrofie - of minder wordt aangemaakt - bij de mildere variant Becker Spierdystrofie. Het dystrofine gen is verantwoordelijk voor de productie van meerdere dystrofine isoformen die voorkomen in verschillende lichaamsweefsels, namelijk in de spieren, de nieren, het oog en het brein. Het wetenschappelijk onderzoek heeft de laatste jaren zich beziggehouden met het onderzoeken van het verband tussen de verstoorde productie van dystrofine in het brein en het vaker voorkomen van neurocognitieve (d.w.z. leer- en denkproblemen) en gedragsproblemen. De exacte rol van dystrofine in cognitie en gedrag blijft onduidelijk. Het doel van dit proefschrift was om de relatie tussen dystrofine in het brein en de veelvoorkomende gedrags- en neurocognitieve problemen verder te beschrijven. Tevens hebben we middels een literatuurstudie de in de wetenschappelijke literatuur gebruikte psychologische meetinstrumenten in kaart gebracht.

Tot slot, er is nog weinig onderzoek verricht naar behandelingen om de gevolgen van de gedrags- en neurocognitieve problemen te verminderen. In dit proefschrift onderzoeken we twee behandelingen bij jongens met DMD, namelijk een psychologische interventie (cognitieve werkgeheugentraining) en een medicamenteuze behandeling ter verbetering van de aandacht (methylfenidaat).

In hoofdstuk 2 vergelijken we het neurocognitief en gedragsmatig functioneren van jongens met DMD met dat van jongens die een andere genetische aandoening hebben, namelijk het neurofibromatose type 1 (NF1). NF1 wordt veroorzaakt door een mutatie in het NF1 gen dat verantwoordelijk is voor de productie van het eiwit neurofibromin. Dit eiwit komt voor in verschillende lichaamsweefsels, onder andere in neuronen en glia cellen in het brein. De huidige NF1 literatuur heeft zich de laatste jaren beziggehouden met het verband tussen de verstoorde productie van het eiwit neurofibromin in het brein en het voorkomen van cognitieve- en leerproblemen.

Uit de resultaten van ons onderzoek blijkt dat de jongens met DMD niet verschillen van jongens met NF1 voor wat betreft intelligentie en specifieke neurocognitieve functies zoals intellectuele vaardigheden, werkgeheugen, visueel-ruimtelijke vaardigheden en 
volgehouden aandacht. Op basis van gedragsvragenlijsten die zijn ingevuld door ouders en leerkrachten, kunnen geen significante verschillen in het voorkomen van gedragsproblemen vastgesteld worden tussen de DMD- en de NF1-groep. Concluderend, er zijn overeenkomstige neuropsychologische profielen bij de DMD- en NF1 groep. Dit wekt de suggestie dat gedrags- en neurocognitieve problemen bij deze genetische aandoeningen niet alleen veroorzaakt worden door een genetische mutatie, voortvloeiend uit de afwezigheid van één specifiek eiwit.

In hoofdstuk 3 beschrijven we in een longitudinaal retrospectief onderzoek de neurocognitieve gegevens van verbale geheugenspanne en informatieverwerkingssnelheid van jongens met DMD. Bovendien hebben we de mogelijke aanwezigheid van een genotype-fenotype relatie onderzocht. Hiermee wordt bedoeld een verband tussen de erfelijke (DNA) eigenschappen en de uiterlijke kernmerken van een individu.

De resultaten van twee meetmomenten naar verbalegeheugenspanne en verwerkingssnelheid voor de totale groep jongens met DMD werden vergeleken. Vervolgens werd de totale groep opgedeeld in subgroepen, om het verband tussen het dystrofine isoform in het brein (Dp140) en de neurocognitieve gegevens te onderzoeken. Op beide meetmomenten presteerde de totale groep laag op de verbalegeheugenspannetaak, terwijl de verwerkingssnelheid normaal was. Gegevens van de subgroepen laten na analyse zien dat jongens waarbij het dystrofine isoform in het brein (Dp140) ontbreekt, een lagere prestatie leveren in verbalegeheugenspanne en verwerkingssnelheid dan jongens waarbij dit eiwit intact is.

Concluderend zien we bij jongens met DMD een stagnatie in de ontwikkeling van verbalegeheugenspanne, die onafhankelijk blijkt te zijn van problemen in verwerkingssnelheid. Deze stagnatie lijkt nadrukkelijker aanwezig te zijn bij jongens, waarbij het dystrofine isoform (Dp140) in het brein mist.

In hoofdstuk 4 beschrijven we de resultaten van neurocognitieve testen en gedragsvragenlijsten op basis van zelf-rapportage van volwassen patiënten met BMD.

De resultaten van de BMD-groep werden vergeleken met normdata. Daarnaast werden correlaties onderzocht tussen de uitkomsten van de neurocognitieve testen en gedragsvragenlijsten en de meetscores die de ernst van ziekte weergeven (in het bijzonder door gestandaardiseerde testen die informatie geven over spierkracht en bewegen zoals de 10-meter-ren-/looptest en de Performance Upper Limb Test 1.2.).

De resultaten lieten zien dat de (geschatte) intellectuele vaardigheiden, het verbale geheugen, de verwerkingssnelheid en de executieve functies van de BMD groep laaggemiddeld zijn ten opzichte van de normdata. Echter, klinisch gezien vallen de uitkomsten binnen de normale waardes. De gedragsvragenlijsten toonden geen 
afwijkende scores voor de BMD groep. Voorts werd voor geen enkele persoon een neuro-ontwikkelingsdiagnose gemeld zoals een autisme-spectrumstoornis (ASD) of een obsessief-compulsieve stoornis (OCD). Slechts één patiënt was gediagnosticeerd met een aandachts-deficiëntie/hyperactiviteitsstoornis (ADHD). De scores voor de ernst van de ziekte en de resultaten van de neurocognitieve testen en de vragenlijsten correleerden niet significant.

Onze bevindingen tonen aan dat bij volwassen met BMD geringe cognitieve problemen kunnen voorkomen, onafhankelijk van de ernst van de ziekte. Voor de klinische zorg is het van belang dat clinici op de hoogte zijn van de mogelijke aanwezigheid van deze geringe cognitieve problemen.

In hoofdstuk 5 beschrijven we de gedrags- en neurocognitieve profielen van drie jonge broers met BMD. Deze broers hebben precies dezelfde mutatie in het DMD-gen (van exon 45-48).

De intellectuele vaardigheden van deze broers variëren van normaal tot hoog. Alle drie de jongens presteren moeizaam in verwerkingssnelheid en rekenvaardigheid. De resultaten van de broers in executief functioneren, werkgeheugen, aandacht en lezen zijn verschillend. Daarnaast worden er verschillen in de cognitieve ontwikkeling gevonden.

Uit deze casuïstische beschrijving kan afgeleid worden dat de gedrags- en neurocognitieve kenmerken van jonge patiënten met BMD ondanks een gelijke mutatie in het DMD-gen en ondanks gelijke omgevingsfactoren, verschillend kunnen zijn.

In hoofdstuk 6 beschrijven we een systematisch literatuuronderzoek naar meetinstrumenten voor het psychosociale en gedragsmatig functioneren van DMD- en BMD-patiënten. Daarnaast beoordelen we de psychometrische kwaliteiten en toepasbaarheid van deze meetinstrumenten.

In 54 wetenschappelijke onderzoeken ( 51 voor DMD, 3 voor BMD) zijn er in totaal 61 meetinstrumenten gebruikt. Daarvan wordt de Child Behavior Checklist het frequentst gehanteerd, ondanks het ontbreken van ziekte-gerelateerde psychometrische informatie. Op basis van de psychometrische bevindingen achten we drie meetinstrumenten geschikt voor het screenen van psychosociale problemen: de Psychosocial Adjustment and Role Skills Scale $3^{\text {rd }}$ edition, de Pediatric Quality of Life Inventory Generic Module en de Life Satisfaction Index for Adolescents with Duchenne Muscular Dystrophy.

De psychometrische kwaliteiten van meetinstrumenten die gebruikt kunnen worden voor het screenen van gedrags- en emotionele problemen, zouden verder geëvalueerd moeten worden, hoewel de Strengths and Difficulties Questionnaire, de Generalized 
Anxiety Disorder-7 item questionnaire en de Patient Health Questionnaire mogelijk bruikbare meetinstrumenten zijn.

Om tot de gouden standaard te komen voor het screenen van gedragsmatig, emotioneel en psychosociaal functioneren van DMD- en BMD-patiënten is verdere evaluatie van de zes bovengenoemde meetinstrumenten geboden. Voor het diagnosticeren van gedragsstoornissen adviseren wij de huidige gouden standaard toe te passen. Dit houdt in dat behandelaars de multi-method, multi-source en multisetting methode in het assessment gebruiken. Voor de DMD- en BMD-populatie is deze werkwijze aan te bevelen, gelet op het hogere risico van comorbiditeiten (oftewel bijkomende aandoeningen).

In hoofdstuk 7 beschrijven we of (1) door computertraining van het cognitieve werkgeheugen dystrofine-geassocieerde problemen van het verbale werkgeheugen van jongens met DMD verminderen, of (2) de effecten van training onmiddellijk na interventie, na 3 en 8 maanden follow-up, waarneembaar zijn, en of (3) training leerproblemen kan verminderen.

We vonden korte-en-lange-termijneffecten op het verbale en visuele werkgeheugen, op schoolse vaardigheden als lezen en rekenvaardigheid, verwerkingssnelheid en logisch redeneren. Bij alle meetmomenten hebben ouders op vragenlijsten minimale veranderingen in executieve functiegedragingen gemeld en leerkrachten rapporteerden geen veranderingen. Daarentegen constateerden we aan de hand van terugkerende ouderrapportages positieve effecten op het werkgeheugen als gevolg van training. We gebruikt daarvoor single case experimental design analyses.

Onze bevindingen tonen positieve effecten van gecomputeriseerde training van het werkgeheugen, zowel op de korte als de lange termijn. Deze effecten zijn ook eerder vastgesteld in onderzoeken bij patiënten met leerproblemen of een ADHD-diagnose, zonder DMD. Dit wekt mogelijk de suggestie dat dystrofine een beperktere rol speelt in de specifieke hersennetwerken van het werkgeheugen dan men verwacht. Echter, een andere verklaring zou kunnen zijn dat compenserende mechanismen alternatieve hersennetwerken voor het werkgeheugen gecreëerd hebben om aldus tekortkomingen op te lossen.

In hoofdstuk 8 beschrijven we met het oog op leerproblematiek de effectiviteit en veiligheid van een medicamenteuze behandeling -bestaande uit een preparaat van methylfenidaat - bij patiënten met DMD en comorbide ADHD.

Op korte termijn werd er bij vier patiënten een vooruitgang in aandacht (concentratie, impulsiviteit en afleidbaarheid) geconstateerd. Bij vier andere patiënten zagen we suboptimale effecten en bij twee patiënten konden we geen effecten rapporteren. 
Op lange termijn zagen we een verbetering in aandacht bij zeven van de tien patiënten. Er werden geen bijwerkingen van de medicijnen gemeld.

Uit de gegevens blijkt dat het preparaat methylfenidaat effectief is voor de behandeling van leerproblemen bij patiënten met DMD en comorbide ADHD. Het verdient aanbeveling bij deze populatie regelmatig een cardiale controle uit te voeren en te monitoren op bijwerkingen (zoals geadviseerd in de landelijke richtlijnen) en neuropsychologische effecten.

Concluderend kunnen we zeggen dat de onderzoeken in deze dissertatie hebben bijgedragen aan nieuwe kennis over de DMD en BMD hersengerelateerde comorbiditeiten. Het (herhaald) evalueren van gedrags- en kennisfuncties en de ontwikkeling van deze comorbiditeiten is van groot belang voor beide spierziekten. Daarnaast zien we nog weinig consensus in het gebruik van psychologische instrumenten bij DMD en BMD. Tevens hebben we in deze dissertatie een eerste aanzet gedaan tot het onderzoeken van interventies gericht op de hersengerelateerde comorbiditeiten bij DMD. Deze resultaten tonen aan dat een psychologische (dwz cognitieve) interventie en medicamenteuze behandeling bestaande uit methylfenidaat de gedrags- en neurocognitieve problemen kunnen verminderen. De rol van diverse mechanismen (o.a. de genetica, neurofysiologische afwijkingen en anatomische afwijkingen van de hersenen) in het voorkomen van en de diversiteit van gedrags- en neurocognitieve problematiek behoeft verder onderzoek. Dit zal het stellen van diagnoses en het opzetten van interventies door zorgprofessionals optimaliseren. 
Addendum 


\section{Impact paragraph}

\section{Introduction}

Duchenne muscular dystrophy (DMD) and Becker muscular dystrophy (BMD) are hereditary neuromuscular disorders covering the spectrum of X-linked dystrophinopathies. Both disorders are caused by mutations in the DMD gene, that encodes multiple dystrophin isoforms in various tissues (e.g. skeletal striated and smooth muscles, kidney, retina, urinary and the brain). Depending on mutation location the production of one or more dystrophin protein isoforms are disturbed. The absence of the full-length dystrophin is responsible for the progressive muscle pathology in DMD. By contrast, in BMD, gene mutations cause variable levels of partially functional dystrophin, resulting in a milder and more variable phenotype ranging from adult onset muscle cramps to severe muscle weakness. An aberrant expression of dystrophin isoforms in the brain has recently been related to the frequently observed comorbid neurocognitive impairments, neurodevelopmental- (e.g. attention-deficit hyperactivity disorder or autism spectrum disorders) and behavioural disorders (e.g. depression or obsessive-compulsive disorder) in DMD and BMD. However, these features differ between patients. Furthermore, since the muscle pathology is progressive, questions arise concerning the neurocognitive features: are these progressively deteriorating as well or do they remain stable over time reflecting a developmental stagnation?

Treatment of muscle functioning currently consists of corticosteroid treatment and physiotherapy for maintaining and strengthening muscles. This is also described in the DMD standards of care. There are some recommendations in the 2018 updated DMD standards of care on treatment of the features i.e. neurocognitive impairments and neurodevelopmental-, and behavioural disorders, but scientific research is lacking. It would be valuable to evaluate neurocognitive or behavioural treatment options, especially when the cognitive impairments develop progressively over time or are caused by a developmental delay and when the behavioural problems negatively affect development and/or quality of life of patients. This paragraph describes the aims and results of this thesis and it relevance for science and practical applications.

\section{Aim, results and conclusions}

Current thesis has focused on the neurocognitive impairments and neurodevelopmental and behavioural disorders in both dystrophinopathies (chapter 2-

5). Furthermore, we evaluated and systematically reviewed assessment tools used to establish behavioural and psychosocial problems in males with DMD (chapter 6). Finally, we explored whether nonmotor treatment options i.e. cognitive training and 
pharmacological treatment used in other (chronic) patient populations may be effective and safe for males with DMD (chapter 7-8).

The findings of present thesis acknowledge that in DMD and BMD, problems may arise in neurocognition, particularly in intellectual functioning, processing speed and verbal short-term memory/ verbal span capacity. This latest problem is suggested to remain present over time in DMD patients (chapter 3). However, no typical neurocognitive or behavioural dystrophinopathy profile was found within our paediatric DMD and BMD males and adult BMD males when they were compared to normative data or patients with other neurogenetic disorders (chapters 2-4-5). With regard to treatment options, results of this thesis display positive effects of cognitive interventions such as working memory training (chapter 7) and pharmacological treatment such as stimulant medication (chapter 8) in paediatric DMD patients with comorbid working memory problems or ADHD. Furthermore, we found that behavioural and psychosocial assessment in DMD and BMD has been performed increasingly in the last two decades. However, the reported prevalence rates of behavioural disorders or psychosocial problems vary extensively. This may depend on (1) the different instruments used by previous studies and (2) a lack of good psychometric properties of the instruments used (chapter 6).

This thesis confirms that both dystrophinopathy disorders are not only characterized by (progressive) myopathy. For now, the name Duchenne muscular dystrophy should be adapted and appointed as Dystrophin Multi-organ Disease. Neurocognitive impairments may be important features as well. It remains unclear whether these impairments progressively deteriorate or whether they are caused by a developmental stagnation due to alterations in maturation. Based on a first longitudinal analyses of findings of DMD patients, we found indications for a developmental stagnation with respect to working memory. Future longitudinal studies are required to further explore this developmental profile. Concerning treatment of the neurocognitive and behavioural features we recommend using evidence-based interventions i.e. cognitive training or psychopharmacological treatment as these may stimulate (academic) development and increase quality of life.

\section{Relevance}

The research described in this thesis can be considered innovative in several ways. Results acknowledge that patients with dystrophinopathies (DMD and BMD) may exhibit neurocognitive impairments and behavioural disorders. In particular impairments in intellectual abilities, processing speed and verbal short-term memory/ 
verbal span capacity (chapter 2-5). These may even remain present over time, suggesting that the cognitive impairments may be caused by a developmental delay. This emphasizes that (re) evaluation of cognitive functioning is important, since these impairments may (eventually) impact academic skills development (i.e. reading and math) and successes.

Results of present thesis displays the effect of training i.e. computerized working memory training on verbal short-term memory impairments in paediatric DMD patients. Improvements were shown in academic skills as well as on parent reports using a SCED design (chapter 7). For treatment of these impairments, health-care professionals may implement this training in standard care when working memory problems are stagnating development. However, generalizations to daily life situations i.e. academic skills and development at school should be further investigated. Previous studies in other populations using computerized cognitive training, show that generalizations to daily life situations can be achieved by using strategies such as explicit strategy instruction. This promotes using strategies on computerized training tasks and it additionally ensures that patients relate new acquired strategies to relevant daily life areas. Future research, should evaluate the combination of training and explicit strategy instruction when treating cognitive impairments.

Furthermore, results of present thesis have shown that stimulant medication may be effective and safe for paediatric DMD males with attention problems. Recent updated standards recommend the use of this pharmacological treatment in patients with attention problems. However, since these patients are more at risk for cardiovascular problems, it is important that treatment efficacy and safety is evaluated beforehand. Our study (chapter 8) describes scientific data showing that simulant medication i.e. methylphenidate considerably improves attention in DMD patients, without major medical side effects (in cardiac status). Though, future studies should evaluate the efficacy and safety of stimulant medication in this population, using a randomized controlled trial or single case design with additional and more complete neuropsychological outcomes.

Finally, results of the review study in the present thesis show that neurodevelopmental- , behavioural disorders as well as psychosocial problems of DMD and BMD patients have been evaluated frequently by previous studies using various instruments. Our systematic review (chapter 6) gives an overview of all instruments being used and results showed that three instruments: the Psychosocial adjustment and Role Skills Scale $3^{\text {rd }}$ edition (PARS-III), the Paediatric Quality of Life Inventory 
Generic Module (PedsQL GM) and the Life Satisfaction Index for Adolescents with DMD (LSIA) have good psychometric properties and should be implemented in standard psychosocial screening of DMD care. However, unknown psychometric information (e.g. construct validity or test-retest reliability) of these psychosocial instruments should be further evaluated. Concerning behavioural screenings instruments, we encourage researchers to further evaluate the psychometrics and applicability of instruments to determine which should be implemented during standard screening. In case of diagnosing behavioural disorders, our review suggests that researchers and clinicians should not use one instrument for establishing a definite diagnosis, but should apply the multi-method, multi-source, multi-setting approach. Approximately 20-30\% of DMD patients display more than one neurodevelopmental or behavioural disorder and the overlapping symptoms may result in under or overdiagnosis when disorders are diagnosed based on one screening instrument.

\section{Target population}

The findings presented in this thesis are of interest for several target groups. To patients (and their parents/caregivers) with Duchenne or Becker muscular dystrophy and educational professionals, it provides additional information on the presence and variable severity of neurocognitive impairments, neurodevelopmental-, and behavioural problems in both dystrophinopathies. Awareness for cognitive and behavioural disfunctions may lead to increased referrals to health professionals.

All health care professionals (paediatric neurologists, paediatricians, (neuro)psychologists, psychiatrists, rehabilitation physicians, general practitioners, paramedics such as physiotherapist) treating patients with DMD or BMD are important stakeholders as well. They are the ones who should be aware of the risk for developing neurocognitive impairments, neurodevelopmental- and behavioural disorders during different life stages and should inform patients and caregivers appropriately. Early screening and diagnosis of these problems may contribute to better academic achievements and an increased health-related quality of life of DMD and BMD patients. Additionally, psychologist and other professionals may take the results of our review in account when applying behavioural screening and assessment. Results of this thesis also informs health care professionals about psychological or pharmacological interventions and their possible (side) effects, when paediatric DMD patients display verbal working memory or attention problems. 
The findings from this thesis are also relevant for researchers in the DMD and BMD field, as they may shed more light on the variable severity and development of neurocognitive- and behavioural problems in DMD and BMD. Our data may encourage researchers to further assess the role of abnormal brain functioning (i.e. aberrant brain dystrophin expression or brain structure abnormalities) on neuro- cognition, development and behaviour. Additionally, researchers may be encouraged to further evaluate (1) psychometrics of behavioural and psychosocial instruments for the DMD and BMD population and (2) treatment effects of other known evidence-based interventions. For instance the effectiveness of pharmacological treatment i.e. selective serotonin-reuptake inhibitors in DMD or BMD patients with comorbid anxiety, depression or obsessive-compulsive disorders. Furthermore, it is valuable to know that research designs such as single case experimental design studies may be useful tools to analyse treatment effects in relatively small groups of patients with dystrophinopathies. It is often difficult to include large DMD or BMD sample sizes and follow them longitudinally. Patients are also frequently contacted by several researchers, which may overburden them. SCED designs may diminish these problems.

\section{Implementation and future work}

The knowledge derived from the studies presented in this thesis will be used for continuation of research on one hand and implemented in health care on the other hand. Researchers should connect (longitudinal) neurocognitive and behavioural data to neurophysiological and neuroimaging alterations, to assess the relation of the underlying aetiology and neuro- cognitive, developmental and behavioural disfunctions in both dystrophinopathies. With respect to diagnostic work-up, additional evaluation of psychometrics of psychosocial screening instruments (i.e. the PARS-III, PedsQL GM and LSIA) and behavioural screening instruments should be performed to determine a gold standard protocol. With respect to interventions researchers can further focus on the efficacy and safety of known evidence-based psychological or pharmacological interventions for DMD and BMD patients.

In clinical practice the results of this thesis can by implemented by health care professionals during diagnostic work-up of cognitive and behavioural functioning. Furthermore, the review of instruments being used as described in this thesis may encourage health care professionals (1) to adequately use screening instruments and (2) to implement the multi-method, source, setting approach in case of definite diagnostics. The intervention i.e. computerized working memory training can be implemented during DMD care by educational and health care professionals when 
DMD patients exhibit problems in this domain. Furthermore, prescribing health care professionals may implement the use of stimulant medication such as methylphenidate in DMD patients with comorbid attention problems, with awareness for both the neuropsychological and medical (side) effects, in particular to cardiovascular adverse events.

\section{Activities and products}

The findings of this thesis have been presented at national and international conferences. These conferences were attended by health care professionals and researchers. Moreover, results have been discussed at (clinical) symposia and expert meetings in clinical settings. Some results have for instance been presented to a network of health care professionals (psychologists ad educationalists) working with males with DMD in special education schools, rehabilitation centres and university hospitals in the Netherlands and Belgium. New insights were communicated to health care professionals, researchers as well as to patients and their caregivers at (1) the Dutch parent platform, (2) the annual organised symposia (Duchenne Parent Project) and (3) working conferences of the Duchene Center Netherlands (which is a collaboration of the Leiden University Hospital, Radboud University Hospital, Maastricht University Hospital and Kempenhaeghe Center of Neurological Learning Disabilities. Results of this thesis are also available at the website of the Duchenne Center Netherlands. 


\section{Dankwoord}

Het is zover, ik ben toegekomen aan het dankwoord, het laatste stuk dat het proefschrift compleet maakt. De afgelopen 4 jaar zijn voorbij gevlogen. Zonder de inspanningen van een aantal mensen was dit proefschrift niet tot stand gekomen. Daarom wil ik graag alle mensen bedanken die hieraan een bijdrage hebben geleverd.

Allereerst wil ik mijn $1^{\mathrm{e}}$ promotor bedanken. Beste professor Vles, bedankt voor al uw inspanningen, adviezen, hulp en het delen van uw klinische en wetenschappelijk kennis en ervaringen. Uw betrokkenheid tijdens mijn traject was zeer waardevol! Het was voor mij een uitdaging om de neurologie, genetica en neuropsychologie samen te voegen en te integreren. Dankzij uw kritische blik zocht ik aspecten grondig uit, tot ik overtuigd was van de bevindingen. Naast hard werken, nam u ook de tijd om stil te staan bij behaalde successen of het bespreken van persoonlijke zaken en hoogtepunten. Beste prof. Vles, dank voor uw hulp en de fijne samenwerking gedurende mijn traject. Ik ben zeer vereerd dat ik u laatste promovenda ben geweest.

Mijn $2^{\mathrm{e}}$ promotor, dr. Hendriksen, beste Jos, ook jou wil ik bedanken voor de leerzame tijd. Jouw toewijding voor het verbeteren van de zorg en kwaliteit van leven van jongens en jonge mannen met Duchenne of Becker is inspirerend. Bedankt voor het delen van jouw neuropsychologische kennis over Duchenne en Becker, alsook de algemene neuropsychologie van kinder-en jeugdigen. Dank voor de fijne samenwerking, je tijd, hulp, adviezen en kansen die hebben bijgedragen aan mijn ontwikkeling als (neuro)psycholoog.

Mijn co-promotor, dr.Klinkenberg, beste Sylvia, ook jou wil ik graag bedanken voor je begeleiding. Tijdens de wekelijkse autoritjes naar Kempenhaeghe bespraken we de nodige werkgerelateerde obstructies alsook persoonlijke hoogte en dieptepunten. Je betrokkenheid naar patiënten is zeer inspirerend. Dank voor je betrokkenheid, hulp en adviezen tijdens mijn traject!

Beste professor dr. Faber, beste Karin, ook jou wil ik graag bedanken. Tijdens onze gezamenlijke DMD team vergadering dacht je mee en gaf je adviezen hoe ik aspecten het beste kon aanpakken of oplossen. Ons uitstapje samen met Judith en Isis naar Kopenhagen, is een mooie herinnering. Naast werktijd en support was er ruimte voor ontspanning. Bedankt voor de fijne samenwerking! 
Beste dr. Hoogland, beste Govert, ook jou wil ik bedanken voor je interesse en adviezen tijdens de DMD-team vergaderingen. Dank voor de fijne samenwerking.

Hartelijk dank aan alle leden van de beoordelingscommissie, prof. dr. R. van Oostenbrugge (voorzitter), prof. dr. R. Ponds, prof. dr. C. Stumpel, prof. dr. N. Goemans en prof. dr. H. Swaab voor het lezen en beoordelen van mijn proefschrift. In het bijzonder wil ik prof. dr. R. Ponds nog bedanken, gezien hij mij 4 jaar geleden heeft geïntroduceerd als PhD kandidaat bij prof. dr. Vles en dr. Hendriksen.

Een bijzonder woord van dank gaat uit naar alle jongens en (jonge) mannen met Duchenne of Becker spierdystrofie en hun ouders, verzorgers en leerkrachten die geparticipeerd hebben binnen de onderzoeken van dit proefschrift. Jullie bijdrage is onmisbaar! Ook wil ik graag Spieren voor Spieren en Duchenne Parent Project bedanken voor het financieel mogelijk maken van dit onderzoek.

Judith, mijn duo-partner op het project. Onze innige symbiose had ik niet willen missen. Je had de weg al even verkend, voordat ik aan de slag ging als je duo-partner. Met jou kon ik sparren over studie-gerelateerde kwesties en zoveel meer!! Bedankt dat je ook de tijd nam om bijna 1000 abstracts te screenen voor het review, kritisch te kijken naar geschreven stukken en mij uitleg te geven over medische kwesties. Wat ben ik blij dat we samen twee reisjes hebben gemaakt. Ook hier waren we een goed duo-team, zelfs als het ging om de tijdsverdeling m.b.t. badkamergebruik! Bedankt voor alles!

Ook hartelijk dank aan alle (voorheen GWD en momenteel DMP) mensen van Kempenhaeghe die mij geholpen hebben of interesse hebben getoond in mijn traject. In het bijzonder wil ik de volgende mensen bedanken, Irene Gijsselhart dank voor je hulp bij het opzetten van de search voor het review, Lucia Wilbers dank voor je hulp bij het te woord staan van patiënten voor onderzoek, Christie Stals, Astrid Engelen en Annemarie Buermans bedankt voor het altijd zoeken van een fijne werkplek. Astrid, fijn dat jij mij geholpen hebt met het wegwerken van die enorme stapel van patiënteninformatie!

Ook andere GWD/DMP collega's wil ik graag bedanken. Allereerst José, helaas werkte je de laatste 2 jaren van mijn traject niet meer bij Kempenhaege, maar wat heb ik veel van jou geleerd! Ik was een groentje in NPO diagnostiek bij kinder-en jeugdigen. Fijn dat jij hebt mij zoveel over de diagnostiek hebt geleerd. Wat was het super om samen met jou het project van de werkgeheugentraining vorm te geven. Bedankt dat ik altijd bij je binnen kon lopen ongeacht het werk of privézaken betrof! Ook Ellen, wil ik graag 
bedanken! Ook jouw vertrek vond ik jammer! Onze tripjes naar Kempenhaeghe waren altijd zo inspirerend. Ik leerde veel van je klinische, alsook wetenschappelijke ervaringen. Wat mooi dat ook tijdens een van onze tripjes het SCED design van de werkgeheugentraining tot stand is gekomen. Ik ben je daar zeer dankbaar voor! Je luisterend oor was altijd prettig of dat nu was tijdens onze autotripjes, op locatie of tijdens onze high-tea / lunchmomenten. Ontzettend bedankt voor alles.

Sandra, wat een mooi project hebben wij samen doorlopen. Ik ben je dankbaar voor je uitgebreide overlegmomenten, waarin we eerst aandacht schonken aan onze privézaken en ons daarna volledig richtte op het manuscript. Ik heb veel geleerd van jouw expertise in de kinder-en jeugd neuropsychologie. Het leidde tot kritische besprekingen van onze resultaten, bedankt daarvoor!

Andere GWD/DMP-collega's, Dirk, Ella, Marjolein, Marije en Christine ook bedankt voor jullie interesse en hulp! Wat fijn dat ik jullie mocht lastig vallen als ik iets nodig had uit Kempenhaeghe en zelf in Maastricht werkzaam was.

Alle (oud) collega's van de afdeling Psychiatrie en Neuropsychologie in Maastricht wil ik ook graag bedanken voor de fijne en gezellige werksfeer. Bedankt voor jullie interesse in mijn traject! Danielle Moens, Ankie Hochstenbach en Desiree Serpenti, bedankt dat ik jullie altijd laagdrempelig kon benaderen en dat ik op jullie kon rekenen. Nico en Ron, bedankt dat ik jullie 24/7 kon lastigvallen met alle ICT-gerelateerde vragen. Vaak heb ik gezegd en gedacht, als ik Nico en Ron toch niet had...! Zonder jullie hulp is uitvoeren van onderzoek praktisch onmogelijk!

Alle andere collega's in Maastricht wil ik ook graag bedanken. Bianca en Sander, dank voor jullie hulp en tijd als ik vastliep met statistiek en dringende vragen had. Jullie antwoorden gaven verdieping of zorgde ervoor dat ik kon vertrouwen op mijn analyses.

Lieve Isis, wat is het toch altijd gezellig als we tijd hebben om samen koffie te drinken en te kletsen over PhD zaken, huizen en onze liefde voor het Scandinavisch interieur. In Kopenhagen waren we in onze nopjes! Je support als ik weer eens zenuwachtig was voor een presentatie was zeer prettig! Gelukkig zitten we nog bij elkaar in de buurt en zijn onze lunch / koffie momentjes geen verleden tijd.

Collega's van het Duchenne Centrum Nederland, het LUMC en Radboud UMC wil ik ook graag bedanken voor de hulp, interesse en bijdrage aan mijn projecten. In het bijzonder, dank aan Merel, Saskia en Yvonne voor onze fijne samenwerking en hulp bij 
het includeren van patiënten voor het Optimale Zorg project. Nathalie en Zaïda, ook jullie bedankt voor de fijne samenwerking. Ik heb veel van jullie geleerd als collega's binnen het DMD en BMD onderzoek. Zaïda, onze ontelbare skype/tel of persoonlijke overlegmomenten in het heerlijke koffietentje in Den Bosch (met overheerlijke bananencake) zorgde ervoor dat we samen een mooi eindresultaat hebben neergezet (versie 5 of 6 .. of 10 zitten we nu al volgens mij?). Super bedankt voor de leerzame en gezellige momenten! Beste Erik, dank voor de fijne samenwerking tijdens ons gezamenlijk BMD project.

Nieuwe collega's van Adelante, ook jullie wil ik meenemen in dit dankwoord. Jullie zorgen voor een fijne, prettige en ook (te) gezellige werkomgeving! Ik kijk uit naar onze gezamenlijke toekomst!

Lieve Elles, Anne (op jullie kom zo nog terug), Niky en Liselot (coffee en winelovers!). Wij waren diegene die na de stage periode van het MUMC overbleven. Bedankt voor die gezellig tijd samen en wat fijn dat we nog steeds contact hebben en onze lunch / diner momenten blijven voortzetten. Fijn dat er ook altijd ruimte is om te sparren over werkgerelateerde kwesties!

Lieve (ex-roomies) en paranimfen, Elles en Anne, wat ik ben blij en trots dat jullie mij steunen tijdens de grote dag! Lieve Elles, inmiddels kennen we elkaar alweer 6 jaar. Samen zijn we begonnen als stagiaires en werkten we aan de CASPER-studie. Daarna mocht ik als collega nog verder meewerken aan jouw mooie studie en wat hebben we veel uren samen doorgebracht! Onze gezamenlijke liefde voor Sky radio Christmas duurt nog voort, ondanks dat we nu niet meer een kamer delen. Lieve Anne, wij kennen elkaar alweer 10 jaar! Destijds zijn we samen gestart met de bachelor Gezondheidswetenschappen, vervolgens de master Neuropsychologie en geëindigd als promovenda collega's en roomies! Samen met Elles, delen we onze Christmas liefde. Ik denk dat menige jaloers was op ons kantoor tijdens de feestdagen. Het leekt net op Magisch Maastricht, maar dan 2.0 versie. Lieve meiden, bedankt voor jullie support, adviezen en gezelligheid! Ik had het niet willen missen!!!

Lieve vriendinnen, vrienden en (schoon) familie. Ook jullie bedankt voor jullie interesse naar de voortgang en inhoud van mijn promotie. Ik hoop dat jullie met dit boekje meer zicht hebben op mijn traject. In het bijzonder een woord van dank aan mijn oom Jan Coumans voor zijn feedback op de stellingen en Nederlandse samenvatting. 
Helaas heb ik tijdens de afgelopen 4 jaar ook afscheid moeten nemen van dierbaren. Opa en oma Wolder, oma Sint-Pieter, Peetnoonk Sjo en tante Marij, het geit uuch good dao bove!!

Leeve Bompa en Bomma, ouch al zeet geer al eve neet mie onder us. lech wil uuch toch nog bedaanke. Wat zeet geer ' $n$ inspiratiebron gewees. Via uuch höbbe veer gelierd dat hel wèrke nudeg is wèl men örgens koume. Bedaank veur al wat geer altied höb gedaon veur us!

Leeve oma Lenie, wat is ' $t$ bezunder dat iech dit momint nog met uuch maag deile!

Leeve (tant) Kayleigh, sinds eus 4 e jaor zien veer al bevrund en wat bin iech dao gruuts op. Bedaank veur dien intresse, support in mien promotie en alle leuke dinger die veer same höbbe gedaon, die zörgde veur de nudege aofleiing!

Leef breurs, Romain en Julien, wat fijn dat geer altied ' $r$ waort en zeet veur miech! lech weet dat iech altied op uuch kin trökvalle! Merci daoveur!! Leeve Kimberly bedaank veur dien hölp bij het oontwerpe vaan de kaf! Leeve Jeanot, wat bin iech gruuts dat iech dien ierste kopvoeterteikening aon ederein maag laote zien!

Leeve mama en papa, ouch geer wil iech bedaanke! Papa, wat fijn dat us kontak zoe is touwgenome de letste jaore! lech bin blij dat iech bij diech terech kin es het nudeg is. Leeve mama, dien leefde es mam, mer ouch dien passie veur de kinder-en jäögontwikkeling höbbe gebrach boe iech noe bin. De mominte dat iech kaom veur advies of gewoen veur ' $n$ luusterend oer, höbbe meujeleke mominte ' $n$ stök dragelijker gemaak. lech bin diech daankbaar dat stiech altied veur miech klaor steis en ouch zörgs veur de nudege aofleiiing mer zeker ouch geruststèlling.

Tot slot, leefste (papa) Stef. Wat höbbe veer in bekans 6 jaor tied väöl dinger metgemaak. Veer zien gaon samenwoene, höbbe väöl gereis, same ' $n$ hoes gekoch en verbouwd, same hiel väöl gelache mer ouch same mote jenke en dierbare mote aofgeve. Noe höbbe veer same eus wönder Sepp. lech wil diech bedaanke veur de ruimte die stiech miech gaofs veur mien ontwikkeling, dien adviezen en dien support es iech weer eins in paniek raakte op die (zwarte) pieste.... Het is fien um te wete dat stiech ' $r$ altied veur miech bis! lech loer oet nao euze touwkoms met euze Sepp!! 
Addendum 


\section{Curriculum Vitae}

Danique Mariëtte Josephine Hellebrekers was born on October 7, 1991 in Maastricht, the Netherlands. After graduating from high school (Porta Mosana College, Maastricht) in 2009, she started with her bachelor of Nursing at Hogeschool Zuyd and finished her propaedeutic year in 2010. In 2010 she started to study Health Medicine \& Life Sciences, track mental health at Maastricht University and she graduated in 2013. In 2013 Danique started with the Master Neuropsychology at Maastricht University and graduated in 2015. She performed a research internship on the CASPER study at the Department of Psychiatry and Neuropsychology of

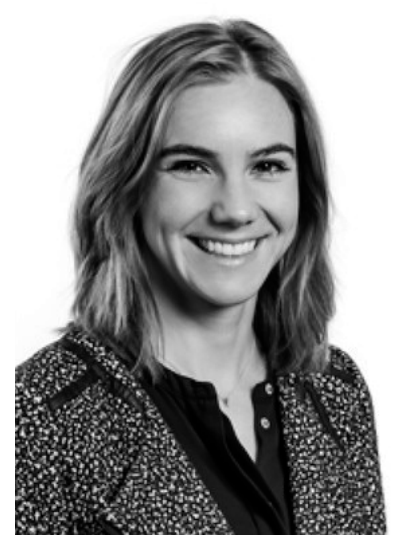
Maastricht University. Additionally, she performed a clinical internship at the Memory Clinic of Maastricht University and acquired her psychodiagnostic registration (BAPD). In 2015 she graduated and started working as a psychologist at Elkerliek Ziekenhuis, Helmond. Thereafter she worked as a research assistant at the Department of Psychiatry and Neuropsychology and additionally started in 2016 as a psychologist at Zuyderland Zorgcentra. In 2016, Danique started working as a PhD candidate on the Duchenne and Becker project at the Department of Neurology of Maastricht University and the Centre of Neurological Learning Disabilities of Kempenhaeghe under supervision of prof. dr. J.S.H. Vles, dr. J.G.M. Hendriksen and dr. S. Klinkenberg. Currently, Danique is working as a psychologist at Adelante in Maastricht University Medical Centre+ (MUMC+).

Danique Mariëtte Josephine Hellebrekers is geboren op 7 oktober 1991 te Maastricht, Nederland. In 2009 behaalde ze haar HAVO diploma aan het Porta Mosana College te Maastricht. Hierna begon Danique aan de studie HBO-verpleegkunde en behaalde haar propedeuse in 2010. Vervolgens startte Danique met de bachelor Gezondheidswetenschappen aan de Universiteit Maastricht. In het tweede studiejaar koos zij voor de richting Geestelijke Gezondheidszorg. De bachelor werd afgerond in 2013. In hetzelfde jaar begon Danique met de Master Neuropsychology aan de Universiteit van Maastricht. Een combinatiestage in onderzoek en kliniek was onderdeel van deze master. Het onderzoek was gericht op de CASPER studie bij de afdeling Psychiatrie en Neuropsychologie van de Universiteit Maastricht. De klinische stage vond plaats bij de geheugepoli in MUMC+. Ten tijde van de klinische stage 
behaalde Danique tevens de registratie psychodiagnostiek (BAPD). Begin 2015 studeerde Danique af en startte als psycholoog in het Elkerliek ziekenhuis te Helmond. Vervolgens is Danique gaan werken bij de afdeling Psychiatrie en Neuropsychologie van de Universiteit Maastricht als onderzoeksassistente. Tevens startte Danique als psycholoog bij Zuyderland Zorgcentra in 2016. In het najaar van 2016 is Danique begonnen als promovenda op het Duchenne en Becker project bij de afdeling Neurologie van de Universiteit Maastricht en het centrum voor Neurologische Leer en Ontwikkelingsstoornissen van Kempenhaeghe onder supervisie van prof. dr. J.S.H. Vles, dr. J.G.M. Hendriksen en dr. S. Klinkenberg. Momenteel is Danique werkzaam als psycholoog bij Adelante, locatie MUMC+. 


\section{List of publications and presentations}

\section{International journals}

Hellebrekers, D.M.J, Doorenweerd, N., Sweere, D.J.J., van Kuijk, S.M.J., Aartsma-Rus, A. M., Klinkenberg, S., Vles, J.S.H., \& Hendriksen, J.G.M. (2020). Longitudinal follow-up of verbal span and processing speed in Duchenne muscular dystrophy. European Journal of Paediatric Neurology, 28, 120-126.

Hellebrekers, D. M.J., Lionarons, J.M., Faber, C.G., Klinkenberg, S., Vles, J.S.H., \& Hendriksen, J.G.M. (2019). Instruments for the Assessment of Behavioral and Psychosocial Functioning in Duchenne and Becker Muscular Dystrophy; a Systematic Review of the Literature. Journal of Pediatric Psychology, 44(10), 1205-1223.

Hellebrekers, D.M.J., Vles, J.S.H., Klinkenberg, S., Hendriksen, J.G.M. (2020). The neurocognitive and behavioral profiles of three brothers with Becker muscular dystrophy. Child Neurology Open,

Lionarons, J.M. ${ }^{*}$, Hellebrekers, D.M.J. , Klinkenberg, S., Faber, C.G., Vles, J.S.H., \& Hendriksen, J.G.M. (2019). Methylphenidate use in males with Duchenne muscular dystrophy and a comorbid attention-deficit hyperactivity disorder. European Journal of Paediatric Neurology, 23(1), 152-157.

\section{Submitted to international journals}

Hellebrekers, D.M.J., van Abeelen, S.A.M., van Kuijk, S.M.J., Laridon, A.M., Klinkenberg, S., Hendriksen, J.G.M., Vles, J.S.H. Cognitive and behavioural functioning in two neurogenetic disorders; how different are these aspects in Duchenne muscular dystrophy and Neurofibromatosis type 1 ?

Hellebrekers, D.M.J., Wirken, J.M.A., Lionarons, J.M., van Kuijk, S.M.J., Klinkenberg, S., Vles, J.S.H., Hendriksen, J.G.M. Computerized working memory training in males with Duchenne muscular dystrophy: a single case experimental design study.

\section{Drafts}

Koeks, Z. , Hellebrekers, D.M.J. ,, van de Velde, N.M., Alleman, I., Spitali, P., van Duyvenvoorde, H.A., Verschuuren, J.J.G.M., Hendriksen, J.G.M. ${ }^{*}$, Niks E.H. ${ }^{*}$ Minor cognitive impairments in adult males with Becker muscular dystrophy 


\section{Other publications}

Douven, E., Staals, J., Freeze, W. M., Schievink, S. H., Hellebrekers, D.M.J., Wolz, R., ... \& Köhler, S. (2020). Imaging markers associated with the development of post-stroke depression and apathy: Results of the Cognition and Affect after Stroke-a Prospective Evaluation of Risks study. European Stroke Journal, 5(1), 78-84.

Hellebrekers, D., Winkens, I., Kruiper, S., \& Van Heugten, C. (2017). Psychometric properties of the awareness questionnaire, patient competency rating scale and Dysexecutive Questionnaire in patients with acquired brain injury. Brain linjury, 31(11), 1469-1478.

Lionarons, J. M., Hoogland, G., Hendriksen, R. G., Faber, C. G., Hellebrekers,D.M.J., Van Koeveringe, G. A., ... \& Vles, J.S.H. (2019). Dystrophin is expressed in smooth muscle and afferent nerve fibers in the rat urinary bladder. Muscle \& Nerve, 60(2), 202-210.

Van Heugten, C., Meuleman, S., Hellebrekers, D., Kruitwagen-van Reenen, E., \& VisserMeily, J. (2018). Participation and the role of neuropsychological functioning in myotonic dystrophy type 1. Journal of Neuromuscular Diseases, 5(2), 205-214.

\section{Published abstracts of conference presentations}

World Muscle Society:

Hellebrekers, D.M.J., Doorenweerd, N., Sweere, D., Kuijk, S., Aartsma-Rus, A.M., Klinkenberg, S., Vles, J.S.H., Hendriksen, J.G,M. (2018). Poster 23. Longitudinal followup of verbal working memory and processing speed in males with Duchenne muscular dystrophy. Neuromuscular Disorders, 28, S38.

Hellebrekers, D.M.J. , Lionarons, J.M. , Klinkenberg, S., Faber, C.G., Hendriksen, J.G.M., Vles, J.S.H. (2018). Poster 22. Evaluation of methylphenidate in males with Duchenne muscular dystrophy and a comorbid attention deficit hyperactivity disorder: a preliminary study. Neuromuscular Disorders, 28, S38.

Hellebrekers, D.M.J., Lionarons, J.M., Wirken, J.M.A., Klinkenberg, S., Vles, J.S.H., Hendriksen, J.G.M. (2019). E-Poster 85. Exploring the trainability of working memory and learning in Duchenne muscular dystrophy using computerized memory training. Neuromuscular Disorders, 29, S177. 
Publications and presentations 
Addendum 


\section{MHENS}

\section{Thesis defenses from MHeNs - School for Mental Health and Neuroscience}

\section{3}

Rob Havermans: Bipolar disorder in daily life; Mood and cortisol responses to naturallyoccurring events. Supervisor: prof.dr. M. de Vries; Co-Supervisor: dr. N. Nicolson.

Véronique Moers-Hornikx: Deep brain stimulation and the cerebellum. Supervisors: prof.dr. J. Vles / prof.dr. Y. Temel; Co-Supervisor: dr. G. Hoogland.

Nicole Veldhorst-Janssen: Intranasal delivery of rapid acting drugs. Supervisors: prof.dr. M. Marcus / prof.dr. C. Neef; Co-Supervisor: dr. P.H. van der Kuy.

Stéphanie Knippenberg: Vitamin D and Multiple Sclerosis: immunological and clinical outcome. Supervisor: prof.dr. J. Cohen-Tervaert; Co-Supervisors: dr. J. Damoiseaux / dr. Y. Bols.

Erik D. Gommer: Dynamic Cerebral Autoregulation: from methodology towards clinical application. Supervisors: prof.dr. W.H. Mess / prof.dr. R.B. Panerai, UK; Co-Supervisor: dr.ir. J.P.H. Reulen.

Olga A.H. Reneerkens: Can PDE inhibition improve cognition ? Translational insights. Supervisor: prof.dr. H.W.M. Steinbusch; Co-Supervisor: dr. J. Prickaerts;

Lyzel S. Elias-Sonnenschein: Clinical and biomarker correlates of genetic risk factors for Alzheimer's disease. Supervisor: prof.dr. F.R.J. Verhey; Co-Supervisor: dr. P.J. Visser.

Diego F. Mastroeni: Epigenetic Dysregulation and the Pathophysiology of of Alzheimer's Disease. Supervisors: prof.dr. H.W.M. Steinbusch / prof.dr. P.D. Coleman, Sun City, Arizona; CoSupervisors: dr. B.P.F. Rutten / dr. D.L.A. van den Hove.

Leonidas Chouliaras: Epigenetic Regulation in Aging and Alzheimer's disease: A translational perspective. Supervisor: prof.dr. H.W.M. Steinbusch; Co-Supervisors:dr. B.P.F. Rutten / dr. D.L.A. van den Hove. 
Liesbeth Knaepen: Perinatal events and altered pain sensitivity in later life. Supervisors: prof.dr. E.A.J. Joosten / prof.dr. D. Tibboel, EUR; Co-Supervisor: dr. J. Patijn.

Marisela Martinez-Claros: Hippocampal plasticity and corticosterone: From dendrites to behaviour. Supervisor: prof.dr. H.W.M. Steinbusch; Co-Supervisors: dr. J.L. Pawluski / dr. J. Prickaerts.

Marcus D. Lancé: A circle of improvement in bleeding management: from laboratory toclinic and back. Supervisors: prof.dr. M.A.E. Marcu / prof.dr. J.W.M. Heemskerk; Co-Supervisor: dr. Y.M.C. Henskens.

Hilde Braakman: Imaging the brain; neuronal correlates of cognitive impairment inchildren with frontal lobe epilepsy. Supervisors: prof.dr. A.P. Aldenkamp / prof.dr. J.S.H. Vles; Co-Supervisors: dr.ir. W.H. Backes / dr. P.A.M. Hofman.

Willem H. van Zwam: Aneurysmal subarachnoid hemorrhage: imaging strategies and costeffectiveness aspects in diagnostic work-up and post-therapeutic follow-up. Supervisors: prof.dr. J.T. Wilmink / prof.dr. J.E. Wildberger; Co-Supervisor:dr. P.A.M. Hofman.

Klara De Cort: The Pathogenesis of Panic Disorder. Supervisors: prof.dr. I. Myin-Germeys / prof.dr. E.J.L. Griez; Co-Supervisors: dr. K.R.J. Schruers / dr. I. Van Diest, Leuven.

Kim van Wijck: Mind the Gap; experimental studies on splanchnic hyperfusion and gastrointestinal integrity loss in man. Supervisors: prof.dr. W.A. Buurman / prof.dr. C.H.C. Dejong; Co-Supervisor: dr. K. Lenaerts.

Yvette Roke: Antipsychotic-induced hyperprolactinemia in children and adolescents with mainly autism spectrum disorders. Prevalence, symptoms, clinical consequencesand genetic risk factors. Supervisors: prof.dr. P.N. van Harten / prof.dr. J.K. Buitelaar (RUN); Co-Supervisor: dr. A. Boot (UMCG).

Fleur Goezinne: Retinal detachment surgery: pre and postoperative prognostic factors. Supervisors: prof.dr. F. Hendrikse / prof.dr. C.A.B. Webers; Co-Supervisor: dr. E.C. La Heij (Amsterdam).

Ralph L.J.G. Maassen: The Merits of Videolaryngoscopy during Glottic Visualisation for Endotracheal Intubation. Supervisors: prof.dr. M. Marcus / prof.dr. A. van Zundert (University of Queensland).

Maria J. de Sousa Guerreiro: The role of sensory modality in age-related distraction. Supervisor: prof.dr. C.M. van Heugten; Co-Supervisor: dr. P.W.M. van Gerven. 
Ine Rayen: Effects of developmental fluoxetine exposure on neurobehavioral outcomes. Supervisor: prof.dr. H.W.M. Steinbusch; Co-Supervisors: dr. J.L. Pawluski / d. T.D. Charlier (Ohio University, USA).

Nynke M.G. Bodde: Psychogenic non-epileptic seizures; a separate disorder or part of a continuum? Supervisors: prof.dr. R. van Oostenbrugge / prof.dr. K. Vonck (UZ Gent); CoSupervisors: dr. R. Lazeron / dr. A. de Louw (Epilepsiecentrum Kempenhaeghe, Heeze).

Alejandro M. Gomez: Novel strategies for making myasthenia less gravis: targeting plasma cells and the neuromuscular junction. Supervisor: prof.dr. M.H. De Baets; Co-Supervisors: dr. M. Losen / dr. P. Martinez-Martinez.

Mohammad S. Rahnama'i: Prostaglandins and Phosphodiesterases in the Urinary Bladder Wall. Supervisors: prof.dr. Ph. Van Kerrebroeck / prof.dr. S. de Wachter (Universiteit Antwerpen); CoSupervisor: dr. G. van Koeveringe.

Mariken B. de Koning: Studying biomarkers in populations at genetic and clinical high risk for psychosis. Supervisors: prof.dr. T. Amelsvoort / prof.dr. J. Booij (AMC).

Fabien Boulle: Epigenetic regulation of BDNF/TrkB signaling in the pathophysiologyand treatment of mood disorders. Supervisors: prof.dr. H.W.M. Steinbusch / prof.dr. L. Lanfumey (Universiteit Parijs); Co-Supervisors: dr. D. van den Hove /dr. G. Kenis.

\section{4}

Iris Nowak-Maes: Tinnitus; assessment of quality of life \& cost-effectiveness. Supervisors: prof.dr. M. Peters / prof.dr. B. Kremer; Co-Supervisors: dr. M. Joore /d. L. Anteunis.

Marjolein Huijts: Cognitive function in patients with cerebral small vessel disease.Supervisor: prof.dr. R.J. van Oostenbrugge; Co-Supervisors: dr. A.A. Duits / dr. J. Staals.

Markus Gantert: Fetal inflammatory injury as origin of long term disease: Lessons from animal models. Supervisors: prof.dr. B. Kramer / prof.dr. L. Zimmermann; Co-Supervisor: dr. A. Gavilanes.

Elke Kuypers: Fetal development after antenatal exposures: Chorioamnionitis and maternal glucocorticoids. Supervisors: prof.dr. B.W. Kramer / prof.dr. H.W. Steinbusch / prof.dr. Suhas G. Kallapur (University of Cincinnati, Ohio, USA).

Pieter Kubben: Ultra low-field strength intraoperative MRI for Glioblastoma Surgery. Supervisor: prof.dr. J.J. van Overbeeke; Co-Supervisor: dr. H. van Santbrink. 
Laura Baijens: Surface electrical stimulation of the neck for oropharyngeal dysphagia in Parkinson's disease: therapeutic aspects and reliability of measurement. Supervisor: prof.dr. B. Kremer; Co-Supervisor: dr. R. Speyer, Townsville.

Janneke Hoeijmakers: Small fiber neuropathy and sodium channels; a paradigm shift. Supervisor: prof.dr. R.J. van Oostenbrugge; Co-Supervisors: dr. C.G. Faber / dr. I.S.J. Merkies.

Stephanie Vos: The Role of biomarkers in preclinical and prodromal Alzheimer's disease. Supervisor: prof.dr. F.R. Verhey; Co-Supervisor: dr. P.J. Visser.

Muriël Doors: The Value of Optical Coherence Tomography in Anterior Segment Surgery. Supervisors: prof.dr. R.M. Nuijts / prof.dr. C.A. Webers; Co-Supervisor: dr. T.T.J.M. Berendschot.

Anneke Maas: Sleep problems in individuals with genetic disorders associated with intellectual disability. Supervisors: prof.dr. I. Curfs / prof.dr. R. Didden.

Sebastiaan van Gorp: Translational research on spinal cord injury and cell-based therapies; a focus on pain and sensorimotor disturbances. Supervisors: prof.dr. B. Joosten / prof.dr. M. van Kleef; Co-Supervisors: dr. J. Patijn / dr. R. Deumens, KU Leuven.

Andrea Sannia: High risk newborns and brain biochemical monitoring. Supervisor:prof.dr. J.S.H. Vles; Co-Supervisors: dr. D. Gazzolo, Alessandria, Italy / dr. A.W.D. Gavilanes.

Julie A.D.A. Dela Cruz: Dopamine mechanisms in learning and memory: Evidence from rodent studies. Supervisors: prof.dr. H.W.M. Steinbusch / prof.dr. R.J. Bodnar, New York; Co-Supervisor: dr. B.P.F. Rutten.

René Besseling: Brain wiring and neuronal dynamics; advances in MR imaging of focalepilepsy. Supervisors: prof.dr. A.P. Aldenkamp / prof.dr.ir. W.H. Backes; Co-Supervisor: dr. J.F.A. Jansen.

Maria Quint-Fens: Long-term care after stroke; development and evaluation of a long-term intervention in primary care. Supervisors: prof.dr. J.F.M. Metsemakers / prof.dr. C.M. van Heugten / prof.dr. M. Limburg, Almere; Co-Supervisor:dr. G.H.M.I. Beusmans.

Veronique Moulaert: Life after survival of a cardiac arrest; the heart of the matter. Supervisors: prof.dr. J.A. Verbunt / prof.dr. C.M. van Heugten / prof.dr. D.T. Wade, Oxford,UK.

Feikje Smeets: The hallucinatory-delusional state: a crucial connection in the psychosissymptom network. Supervisor: prof.dr. J. van Os; Co-Supervisor: dr. T. Lataster. 
Lies Clerx: Alzheimer's disease through the MR-eye; novel diagnostic markers and the road to clinical implementation". Supervisor: prof.dr. F. Verhey; Co-Supervisors: dr. P.J. Visser / P. Aalten.

Sonny Tan: The subthalamic nucleus in Parkinson's disease. Supervisors: prof.dr. Y. Temel / prof.dr. H.W.M. Steinbusch / prof.dr. T. Sharp, Oxford, UK / prof.dr. V. Visser-Vandewalle, Koln.

Koen van Boxem: The use of pulsed radiofrequency in the management of chronic lumbosacral radicular pain. Supervisors: prof.dr. M. van Kleef / prof.dr. E.A.J. Joosten; Co-Supervisor: Assoc. prof.dr. J. van Zundert.

Jérôme Waterval: Hyperostosis cranialis interna. Supervisors: prof.dr. J.J. Manni / prof.dr. R.J. Stokroos.

Sylvie Kolfschoten-van der Kruijs: Psychogenic non-epileptic seizures; the identification of neurophysiological correlates. Supervisors: prof.dr. A.P. Aldenkamp / prof.dr. K.E.J. Vonck, Universiteit Gent; Co-Supervisors: dr. J.F.A. Jansen /dr. R.H.C. Lazeron, Kempenhaeghe.

Wouter Pluijms: Spinal cord stimulation and pain relief in painful diabetic: polyneuropathy, a translational approach. Supervisors: prof.dr. M. van Kleef /prof.dr. E.A. Joosten; Co-supervisor: dr. C.G. Faber.

Ron Handels: Health technology assessment of diagnostic strategies for Alzheimer's disease. Supervisors: prof.dr. F.R.J. Verhey / prof.dr. J.L. Severens (EUR); Co-Supervisor:dr. M.A. Joore / dr. C.A.G. Wolfs.

Evelyn Peelen: Regulatory T cells in the pathogenesis of Multiple Sclerosis: potential targets for vitamin D therapy. Supervisors: prof.dr. R.M.M. Hupperts / prof.dr. J.W. Cohen Tervaert; CoSupervisor: dr. J.G.M.C. Damoiseaux / dr. M.M.G.L.Thewissen, Diepenbeek.

Reint Jellema: Cell-based therapy for hypoxic-ischemic injury in the preterm brain. Supervisors: prof.dr. B.W.W. Kramer / prof.dr. H.W.M. Steinbusch; Co-Supervisor: dr. W.T.V. Germeraad / dr. P. Andriessen, Veldhoven.

Maria Wertli: Prognosis of Chronic Clinical Pain Conditions: The Example of ComplexRegional Pain Syndrome 1 and Low Back Pain. Supervisors: prof.dr. M. van Kleef; Co-Supervisor: dr. F. Brunner, Zürich / dr. R. Perez, VUmc.

Dagmar Zeef: An experimental model of Huntington's disease: Validation \& Stimulation. Supervisors: prof.dr. Y. Temel / prof.dr. H.W.M. Steinbusch; Co-supervisor:Dr. A. Jahanshahi. 
Jeroen Decoster: Breaking Down Schizophrenia into phenes, genes and environment. Supervisors: prof.dr. I. Myin-Germeys / prof.dr. M. De Hert, KU Leuven; Co-Supervisor:dr. R. van Winkel.

Eaja Anindya Sekhar Mukherjee: Fetal Alcohol Spectrum Disorders: exploring prevention and management. Supervisor: prof.dr. L.M.G. Curfs; Co-Supervisor: prof. S. Hollins, St. George's University of London, UK.

Catherine van Zelst: Inside out; On stereotype awareness, childhood trauma and stigma in psychosis. Supervisors: prof.dr. Ph. Delespaul / prof.dr. J. van Os.

Ibrahim Tolga Binbay: Extended Psychosis Phenotype in the Wider Social Environment. Supervisor: prof.dr. J. van Os; Co-Supervisor: dr. M. Drukker.

Frank Van Dael: OCD matters in psychosis. Supervisors: prof.dr. J. van Os / pof.dr. I. MyinGermeys.

Pamela Kleikers: NOXious oxidative stress: from head toe too and back. Supervisors: prof.dr. H.H.H.W. Schmidt / prof.dr. H.W.M. Steinbusch; Co-Supervisor: dr. B. Janssen.

José Luis Gerardo Nava: In vitro assay systems in the development of therapeutic interventions strategies for neuroprotection and repair. Supervisors: prof.dr.med. J. Weis / prof.dr. H.W.M. Steinbusch; Co-Supervisor: dr. G.A. Brook, RWTH Aachen.

Eva Bollen: Cyclic nucleotide signaling and plasticity. Supervisors: prof.dr. H.W.M. Steinbusch / prof.dr. R. D'Hooge, KU Leuven; Co-Supervisor: dr. J. Prickaerts.

\section{5}

Jessica A. Hartmann: A good laugh and a long sleep; Insights from prospective and ambulatory assessments about the importance of positive affect and sleep in mental health. Supervisor: prof.dr. J. van Os; Co-Supervisors: C.J.P. Simons / dr. M. Wichers.

Bart Ament: Frailty in old age; conceptualization and care innovations. Supervisors: prof.dr. G.I.J.M. Kempen / prof.dr. F.R.J. Verhey; Co-Supervisor: dr. M.E. de Vugt.

Mayke Janssens: Exploring course and outcome across the psychosis-continuum. Supervisor: prof.dr. I. Myin-Germeys; Co-Supervisor: dr. T. Lataster. 
Dennis M.J. Hernau: Dopayours is not dopamine: genetic, environmental and pathological variations in dopaminergic stress processing. Supervisor: prof.dr. I. Myin- Germeys; CoSupervisors: prof.dr. F.M. Mottaghy / dr. D. Collip.

Ingrid M.H. Brands: The adaptation process after acquired brain injury Pieces of the puzzle. Supervisors: prof.dr. C.M. van Heugten / prof.dr. D.T. Wade, Oxford UK; Co-Supervisors: dr. S.Z. Stapert / dr. S. Köhler.

Francesco Risso: Urinary and salivary $\mathrm{S} 100 \mathrm{~B}$ monitoring in high risk infants. Supervisor: prof.dr. J.S.H. Vles; Co-Supervisors: dr. D. Gazzolo, Genoa,Italy / dr. A.W.D. Gavilanes.

Alessandro Borghesi: Stem and Progenitor Cells in Preterm Infants: Role in thePathogenesis and Potential for Therapy. Supervisor: prof.dr. L. Zimmermann; prof.dr. B. Kramer; Co-Supervisors: dr. D. Gazzolo, Genoa,Italy / dr. A.W.D. Gavilanes.

Claudia Menne-Lothmann: Affect dynamics; A focus on genes, stress, and an opportunity for change. Supervisor: prof.dr. J. van Os; Co-Supervisors: dr. M. Wichers / dr. N. Jacobs.

Martine van Nierop: Surviving childhood new perspectives on the link between childhoodtrauma and psychosis. Supervisors: prof.dr. I. Myin-Germeys / prof.dr. J. van Os; Co-Supervisor: dr. R. van Winkel.

Sylvia Klinkenberg: VNS in children; more than just seizure reduction. Supervisors:prof.dr. J. Vles / prof.dr. A. Aldenkamp; Co-Supervisor: dr. H. Majoie.

Anouk Linssen: Considerations in designing an adult hearing screening programme. Supervisor: prof.dr. B. Kremer; Co-Supervisors: dr. L. Anteunis / dr. M. Joore.

Janny Hof: Hearing loss in young children; challenges in assessment and intervention. Supervisors: prof.dr. B. Kremer / prof.dr. R. Stokroos / prof.dr. P. van Dijk, RUG; Co-Supervisor: dr. L. Antheunis.

Kimberly Cox-Limpens: Mechanisms of endogenous brain protection; Clues from the transcriptome. Supervisors: prof.dr. J. Vles / prof.dr. L. Zimmermann; Co-Supervisor: dr. A. Gavilanes.

Els Vanhoutte: Peripheral Neuropathy outcome measures; Standardisation (PeriNomS)study part 2: Getting consensus. Supervisors: prof.dr. C. Faber / prof.dr. P. van Doorn; Co-Supervisor: dr. I. Merkies, Spaarne ziekenhuis Hoofddorp. 
Mayienne Bakkers: Small fibers, big troubles; diagnosis and implications of small fiberneuropathy. Supervisors: prof.dr. C. Faber / prof.dr. M. de Baets; Co-Supervisor: dr. I. Merkies, Spaarne ziekenhuis Hoofddorp.

Ingrid Kramer: Zooming into the micro-level of experience: An approach for understanding and treating psychopathology. Supervisor: prof.dr. J. van Os; Co-Supervisors: dr. M. Wichers, UMC Groningen / dr. C. Simons.

Esther Bouman: Risks and Benefits of Regional Anesthesia in the Perioperative Setting. Supervisors: prof.dr. M. van Kleef / prof.dr. M. Marcus, HMC, Qatar / prof.dr. E. Joosten; CoSupervisor: dr. H. Gramke.

Mark Janssen: Selective stimulation of the subthalamic nucleus in Parkinson's disease; dream or near future. Supervisors: prof.dr. Y. Temel / prof.dr. V. Visser-Vandewalle, Keulen / prof.dr. A. Benazzouz, Bordeax, France.

Reina de Kinderen: Health Technology Assessment in Epilepsy; economic evaluations and preference studies. Supervisors: prof.dr. S. Evers / prof.dr. A. Aldenkamp; Co-Supervisor: dr. H. Majoie / dr. D. Postulart, GGZ O-Brabant.

Saskia Ebus: Interictal epileptiform activity as a marker for clinical outcome. Supervisors: prof.dr. A. Aldenkamp / prof.dr. J. Arends, TUE / prof.dr. P. Boon, UniversiteitGent, België.

Inge Knuts: Experimental and clinical studies into determinants of panic severity. Supervisor: prof.dr. I. Myin-Germeys; Co-Supervisor: dr. K. Schruers; Influencing panic.

Nienke Tielemans: Proactive coping post stroke: The Restored4Stroke Self-Management study. Supervisors: prof.dr. C. van Heugten / prof.dr. J. Visser-Meily, UMC Utrecht; Co-Supervisor: dr. V. Schepers, UMC Utrecht.

Tom van Zundert: Improvements Towards Safer Extraglottic Airway Devices. Supervisors: prof.dr. A.E.M. Marcus / prof.dr. W. Buhre / prof.dr. J.R. Brimacombe,Queensland, Australia / prof.dr. C.A. Hagberg.

Tijmen van Assen: Anterior Cutaneous Nerve Entrapment Syndrome Epidemiology and surgical management. Supervisors: prof.dr. G.L. Beets / prof.dr. M. van Kleef / dr. R.M.H. Roumen / dr. M.R.M. Scheltinga, MMC Veldhoven.

Rohit Shetty: Understanding the Clinical, Immunological and Genetic Molecular Mechanisms of Keratoconus. Supervisors: prof.dr. R.M.M.A. Nuijts / prof.dr. C.A.B.Webers. 
Christine van der Leeuw: Blood, bones and brains; peripheral biological endophenotypesand their structural cerebral correlates in psychotic disorder. Supervisor: prof.dr. J. van Os; Co-supervisor: dr. M. Marcelis.

Sanne Peeters: The Idle Mind Never Rests; functional brain connectivity across the psychosis continuum. Supervisor: prof.dr. J. van Os; Co-supervisor: dr. M. Marcelis.

Nick van Goethem: $\alpha 7$ nicotinic acetylcholine receptors and memory processes: mechanistic and behavioral studies. Supervisor: prof.dr. H.W.M. Steinbusch; Co-supervisor: dr. J. Prickaerts.

Nicole Leibold: A Breath of fear; a translational approach into the mechanisms of panic. Supervisor: prof.dr. H.W.M. Steinbusch; Co-supervisors: dr. K.R.J. Schruers / dr. D.L.A. van den Hove.

Renske Hamel: The course of mild cognitive impairment and the role of comorbidity. Supervisor: prof.dr. F.R.J. Verhey; Co-supervisors: dr. I.H.G.B. Ramakers / dr. P.J. Visser.

Lucia Speth: Effects of botulinum toxin A injections and bimanual task-oriented therapyon hand functions and bimanual activities in unilateral Cerebral Palsy. Supervisors: prof.dr. J. Vles; prof.dr. R. Smeets; Co-supervisor: dr. Y. Janssen-Potten, Adelante Hoensbroek.

Yuan Tian: The effects of Lutein on the inflammatory pathways in age-related macular degeneration (AMD). Supervisors: prof.dr. C. Webers; prof.dr. A. Kijlstra, WUR; Co- supervisor: dr. M. Spreeuwenberg; dr. H. Tange.

Peggy Spauwen: Cognition and Type 2 diabetes; the interplay of risk factors. Supervisors: prof.dr. F. Verhey; prof.dr. C. Stehouwer; Co-supervisor: dr. M. van Boxtel

Marc Hilhorst: Crescentic glomerulonephritis in ANCA associated vasculitis. Supervisors: prof.dr. J. Cohen-Tervaert; Co-supervisor: dr. P. van Paassen

Martin Gevonden: The odd one out: exploring the nature of the association between minority status and psychosis. Supervisors: prof.dr. J-P. Selten; prof.dr. J. Booij, Uva; prof.dr. I. MyinGermeys

Bart Biallosterski: Structural and functional aspects of sensory-motor Interaction in the urinary bladder. Supervisors: prof.dr. Ph. Van Kerrebroeck; prof.dr. S. De Wachter, UvAntwerpen; Cosupervisors: dr. G. van Koeveringe; dr. M. Rahnama'i. 
Alexandra König: The use of information and communication technologies (ICT) for the assessment of patients with Alzheimer's Disease and related disorders. Supervisors: prof.dr. F. Verhey; prof.dr. Ph. Robert, Nice, Fr; Co-supervisors: dr. P. Aalten; dr. R. David,Nice. Fr.

Michelene Chenault: Assessing Readiness for Hearing Rehabilitation. Supervisors: prof.dr. M.P.F. Berger; prof.dr. B. Kremer; Co-supervisor: dr. L.J.C. Anteunis.

Anand Vinekar: Retinopathy of Prematurity. Recent advances in tele-medicine screening, risk factors and spectral domain optical coherence tomography imaging. Supervisor: prof.dr. C.A.B. Webers; Co-supervisor: dr. N.J. Bauer

Fleur van Dooren: Diabetes and Depression: exploring the Interface between Pathophysiological and Psychological factors. Supervisors: prof.dr. F.R.J. Verhey; prof.dr. J.K.L. Denollet, UvT; prof.dr. F. Pouwer, UvT; Co-supervisor: dr. M.T. Schram.

Gabriëlla Pons van Dijk: Taekwondo and physical fitness components in middle-aged healthy volunteers; the Sekwondo study. Supervisors: prof.dr. J. Lodder;prof.dr. H. Kingma; Co-supervisor: dr. A.F. Lenssen.

Yara Pujol López: Development and psychoneuroimmunological mechanisms in depression. Supervisor: prof.dr. H.W.M. Steinbusch; Co-supervisors: Dr. G. Kenis; Dr. D.van den Hove; Dr. Aye Mu Myint, München.

Romina Gentier: $\mathrm{UBB}^{+1}$; an important switch in the onset of Alzheimer's disease. Supervisors: Prof. H. Steinbusch; Prof. D. Hopkins; Co-supervisor: Dr. F. van Leeuwen.

Sanne Smeets: Insights into insight: studies on awareness of deficits after acquired brain injury. Supervisor: Prof. C. van Heugten; Prof. R. Ponds; Co-supervisor: Dr. I. Winkens

Kim Beerhorst: Bone disease in chronic epilepsy: fit for a fracture. Supervisor: Prof. A.Aldenkamp; Prof. R. van Oostenbrugge; Co-supervisor: Dr. P. Verschuure.

Alex Zwanenburg: Cerebral and cardiac signal monitoring in fetal sheep with hypoxic-ischemic encephalopathy. Supervisor: Prof. T. Delhaas; Prof. B. Kramer; Co-supervisors:Dr. T. Wolfs; Dr. P. Andriessen, MMC.

Ismail Sinan Guloksuz: Biological mechanisms of environmental stressors in psychiatry. Supervisor: Prof. J. van Os; Co-supervisors: Dr. B. Rutten; Dr. M. Drukker.

Seyed Ehsan Pishva MD: Environmental Epigenetics in mental health and illness. Supervisor: Prof.dr. J. van Os; Co-supervisors: Dr. B.P.F. Rutten; Dr. G. Kenis. 
Ankie Hamaekers: Rescue ventilation using expiratory ventilation assistance; innovating while clutching at straws. Supervisors: Prof.dr. W.F. Buhre; Prof.dr. M. van Kleef.

Rens Evers. 22q11.2 deletion syndrome: intelligence, psychopathology and neurochemistry at adult age. Supervisors: Prof.dr. L.M.G. Curfs; Prof.dr. T. v. Amelsvoort.

Sarah-Anna Hescham. Novel insights towards memory restoration. Supervisor: Prof.dr. Y. Temel; Co-supervisor: Dr. A. Blokland; Dr. A. Jahanshahi.

João P. da Costa Alvares Viegas Nunes. Insulin receptor sensitization improves affectivepathology in various mouse models. Supervisor: Prof.dr. H.W.M. Steinbusch; Co- supervisors: Dr. K-P. Lesch; Dr. T. Strekalova; Dr.B.H. Cline, Oxford.

Yanny Ying-Yee Cheng. Clinical Outcomes After Innovative Lamellar Corneal Transplantation Surgery. Supervisor: Prof.dr. R.M.M.A. Nuijts; Co-supervisor: Dr. J.S.A.G. Schouten.

\section{6}

Oliver Gerlach. Parkinson's disease, deterioration during hospitalization. Supervisor: Prof.dr. R. van Oostenbrugge; Co-supervisor: Dr. W. Weber.

Remo Arts. Intracochlear electrical stimulation to suppress tinnitus. Supervisor: Prof.dr. R.J. Stokroos; Co-supervisor: Dr. E.L.J. Georg.

Mitchel van Eeden. The $€$ - Restore4stroke study: Economic evaluation of stroke care in the Netherlands. Supervisors: Prof.dr.mr. S.M.A.A. Evers;Prof.dr. C.M. v. Heugten; Co-supervisor: dr. G.A.P. van Mastrigt.

Pim Klarenbeek. Blood pressure and cerebral small vessel disease. Supervisor: Prof.dr. R.J. van Oostenbrugge; Co-supervisor: Dr. J. Staals.

Ramona Hohnen. Peripheral pharmacological targets to modify bladder contractility. Supervisor: Prof.dr. Ph.E.V. van Kerrebroeck; Co-supervisors: Dr. G.A. van Koeveringe; Dr. M.A. Sahnama'i; Dr. C. Meriaux.

Ersoy Kocabicak. Deep brain stimulation of the subthalamic nucleus: Clinical andscientific aspects. Supervisors: Prof.dr. Y. Temel; Prof.dr. K. van Overbeeke; Co-supervisor: Dr. A. Jahanshahi. 
Sven Akkerman. Temporal aspects of cyclic messenger signaling in object recognitionmemory; a pharmalogical approach. Supervisor: Prof.dr. H.W.M. Steinbusch; Co-supervisors: dr. J. Prickaerts; dr. A. Blokland.

Anja Moonen. Emotion and Cognition in Parkinson's disease; etiology and neurobiological mechanisms. Supervisor: Prof.dr. F.R.J. Verhey; Co-supervisor: dr. A.F.G.Leentjens.

Anna Schüth. Three-dimensional bladder tissue morphology. Supervisors: Prof.dr. G.A. van Koeveringe; Prof.dr. M. v. Zandvoort, Aachen; Prof.dr. Ph. V. Kerrebroeck.

Elisabeth van der Ven. Ethnic minority position as risk indicator for autism- Spectrum and psychotic disorders. Supervisors: Prof.dr. J.P. Selten; Prof.dr. J. van Os.

Zuzana Kasanova. Environmental reactivity for better or worse; The impact of stress andreward on neurochemistry, affect and behavior across the psychosis continuum. Supervisor: Prof.dr. I. Myin-Germeys, KU Leuven/UM; Co-supervisor: dr. D. Collip.

Danielle Lambrechts. Ketogenic diet therapies; treatment for children and adults withrefractory epilepsy. Supervisors: Prof.dr. H.J.M. Majoie; Prof.dr. J.S.H. Vles; Prof.dr. A.P. Aldenkamp; Cosupervisor: dr. A.J.A. de Louw, Kempenhaghe, Heeze.

Frank van Bussel. Advanced MRI in diabetes; cerebral biomarkers of cognitive decrements. Supervisors: Prof.dr.ir. W.H. Backes; Prof.dr. P.A.M. Hofman; Co-supervisor: dr. J.F.A. Jansen.

Lisa Schönfeldt. Neurostimulation to treat brain injury? Supervisors: Prof.dr. Y. Temel; Prof.dr. S. Hendrikx, Hasselt; Co-supervisor: dr. A. Jahanshahi.

Rianne Geerlings. Transition in patients with childhood-onset epilepsy; a long way toadulthood. Supervisor: Prof.dr. A.P. Aldenkamp; Co-supervisors:dr. A.J.A. de Louw, dr. L.M.C. Gottmer, Kempenhaeghe.

Nele Claes. B cells as multifactorial players in multiple sclerosis pathogenesis: insights from therapeutics. Supervisors: Prof.dr. V. Somers, Hasselt; Prof.dr. R. Hupperts; Co-supervisors: Prof.dr. P. Stinissen, dr. J. Fraussen, Hasselt.

Olaf Schijns. Epilepsy surgery and biomarkers from history to molecular imaging. Supervisors: Prof.dr. J.J. van Overbeeke; Prof.dr. H. Clustermann, Aachen; Co-supervisors:dr. G. Hoogland; dr. M.J.P. v. Kroonenburgh.

Lizzy Boots. Balanced and Prepared; development and evaluation of a supportive e- health intervention for caregivers of people with early-stage dementia. Supervisors: Prof.dr. F.R.J. Verhey; Prof.dr. G.I.J.M. Kempen; Co-supervisor: dr. M.E. de Vugt. 
Wouter Donders. Towards patient-specific (cerebro-) vascular model applications. Supervisors: Prof.dr. T. Delhaas; Prof.dr.ir. F.N. van de Vosse, TUE; Co-supervisor: dr.ir. W. Huberts.

Sizzle Vanterpool. The implications of intrauterine invasion by microbes for placental Pathology and the occurrence of adverse pregnancy outcomes. Supervisor: Prof.dr. B.W. Kramer. Cosupervisors: dr. J.V. Been, Erasmus MC Rotterdam, dr. U von Rango.

Manuela Heins. The Relationship between Social Adversity, Psychosis, and Depressionacross an Individual's Life Span. Supervisor: Prof.dr. I. Myin-Germeys.

Christianus van Ganzewinkel. NEONATAL PAIN; Out of Sight, Out of Mind? Supervisor: Prof.dr. B.W.W. Kramer; Co-supervisor: dr. P. Andriessen, MMC Veldhoven.

Anne-Hilde Muris. Hype or hope? Vitamin D in multiple sclerosis; A clinical and immunological perspective. Supervisor: Prof.dr. R.M.M. Hupperts; Co-supervisor: dr. J.G.M.C. Damoiseaux.

Gerard Bode. The link between ceramide transporters, innate Immunity and Alzheimer'sdisease. Supervisor: Prof.dr. M.H.V. de Baets; Co-supervisors: dr. P. Martinez, dr. M. Losen.

Jo Stevens. Advanced diagnostics and therapeutics for Alzheimer's disease. Supervisor:Prof.dr. M. de Baets; Co-supervisors: dr. M. Losen, dr. P. Martinez-Martinez.

Rosan Luijcks. Stress and pain in muscles and brain; developing psychophysiologicalparadigms to examine stress and pain interactions. Supervisors: Prof.dr. J.J. van Os; Prof.dr.ir. H.J. Hermens, UT; Co-supervisor: dr. R. Lousberg.

M.C. Haanschoten. Towards efficient cardiac surgery - the integrating role of anesthesiology and intensive care. Supervisors: Prof. dr. W. Buhre; Prof. dr. A. van Zundert (Queensland); Cosupervisors: Dr. M.A. Soliman Hamad; Dr. A. van Straten (Catharina zkhs.)

Harmen Jan van de Haar. Microvascular and blood-brain barrier dysfunction in Alzheimer's disease. Supervisor: Prof.dr.ir. W. Backes; Prof.dr. F. Verhey; Co-supervisor:Dr. J. Jansen; Dr.ir. M. v. Osch, LUMC.

Coenraad Itz. Chronic low back pain, considerations about: Natural Course, Diagnosis, Interventional Treatment and Costs. Supervisor: Prof.dr. M. van Kleef; Prof.dr. F. Huygen, EUR; Co- supervisor: Dr. B. Ramaekers.

Willemijn Jansen. The Path of Alzheimer's disease: from neuropathology to clinic. Supervisor: Prof.dr. F. Verhey; Co-supervisors: Dr. P.J. Visser; Dr. I. Ramakers. 
Ligia dos Santos Mendes Lemes Soares. Phosphodiesterase inhibitors: a potential therapeutic approach for ischemic cerebral injury. Supervisor: Prof.dr. H.W.M. Steinbusch; Co-supervisors: Dr. R.M. Weffort de Oliveira, Brazil; Dr. J. Prickaerts

Martijn Broen. Anxiety and depression in Parkinson's disease. Supervisor: Prof.dr. R.J. van Oostenbrugge; Co-supervisors: Dr. A.F.G. Leentjens; Dr. M.L. Kuijf.

Sandra Schipper. Extrasynaptic receptors as a treatment target in epilepsy. Supervisor: Prof.dr. J.H.S. Vles; Co-supervisors: Dr. G. Hoogland; Dr. S. Klinkenberg; Dr. M.W. Aalbers, RUG.

João Casaca Carreira. Making sense of Antisense Oligonucleotides Therapy in Experimental Huntington's disease. Supervisor: Prof.dr. Y. Temel; Co-supervisors: Dr. A.Jahanshahi; Dr. W. van Roon-Mom, LUMC.

Dominique IJff. Trick or Treat? Cognitive side-effects of antiepileptic treatment. Supervisors: Prof.dr. A.P. Aldenkamp; Prof.dr. M. Majoie; Co-supervisors: Dr. J. Jansen; Dr. R. Lazeron, Kempenhaeghe.

Alfredo Ramirez. Neurogenetic approach in neurodegenerative disorders. Supervisors: Prof.dr. B.P.F. Rutten; Prof.dr. H.W.M. Steinbusch; Prof.dr. M.M. Nöthen, University of Bonn.

Nienke Visser. Toric Intraocular lenses in cataract surgery. Supervisor: Prof.dr. R.M.M.A. Nuijts; Co-supervisor: Dr. N.J.C. Bauer.

Jakob Burgstaller. Prognostic indicators for patients with degenerative lumbar spinal stenosis. Supervisor: Prof.dr. M. van Kleef; Co-supervisors: Dr. M.M. Wertli, University ofZurich; Dr. H.F. Gramke.

Mark van den Hurk. Neuronal Identity and Maturation: Insights from the Single-Cell Transcriptome. Supervisors: Prof.dr. H.W.M. Steinbusch; Prof.dr. B.P.F. Rutten; Co- supervisors: Dr. G. Kenis; Dr. C. Bardy, Adelaide.

Maria Nikiforou. Prenatal stress and the fetal gut. Potential interventions to prevent adverse outcomes. Supervisors: Prof.dr. B.W. Kramer; Prof.dr. H.W. Steinbusch; Co- supervisor: Dr. T.G. Wolfs.

Janneke Peijnenborgh. Assessment of cognition, time perception, and motivation in children. Supervisors: Prof.dr. J.S.H. Vles; Prof.dr. A.P. Aldenkamp; Co-supervisors: Dr. J.Hendriksen; Dr. P. Hurks. 
Joany Millenaar. Young onset dementia; towards a better understanding of care needs and experiences. Supervisors: Prof.dr. F. Verhey; Prof.dr. R. Koopmans, RUN; Co- supervisors: Dr. M. de Vugt; Dr. C. Bakker, RUN.

\section{7}

Adriana Smits. Perinatal factors and hearing outcome. Supervisors: Prof.dr. R.J. Stokroos; Prof.dr. B.W. Kramer; Prof.dr. B. Kremer.

Angela Bouwmans. Transcranial sonography in parkinsonian disorders: clear window or blurred vision. Supervisor: Prof.dr. W.H. Mess; Co-promotores: Dr. W.E.J. Weber; Dr. A.F.G. Leentjens.

Björn K. Stessel. Patient centred care after day surgery: scope for improvement. Supervisors: Prof.dr. W. Buhre; Prof.dr. B. Joosten. Co-supervisor: Dr. A.H. Gramke.

Jan Guy Bogaarts. Quantitative EEG and machine learning methods for the detection of epileptic seizures and cerebral asymmetry. Supervisor: Prof.dr. W.M. Mess; Co-supervisor: Dr.ir. J.P.H. Reulen; Dr.ir. E.D. Gommer.

Martin M. Müller. Pregnancy derived products for treatment of perinatal brain injuries. Supervisors: Prof.dr. B.W.W. Kramer; Prof.dr. D. Surbek, Bern; Co-supervisors: Dr. T. Wolfs; Dr. G. Gavilanes.

Daan Ophelders. Novel treatment strategies for the protection of the preterm brain; Rebalancing inflammation and regeneration. Supervisor: Prof.dr. B. Kramer; Co-supervisor: Dr. T. Wolfs; Dr. R. Jellema.

Rosalie van Knippenberg. Experience sampling in dementia care; an innovative intervention to support caregivers in daily life. Supervisors: Prof.dr. F. Verhey; Prof.dr. R.Ponds; Prof.dr. I. MyinGermeys, KU Leuven; Co-supervisor: Dr. M. de Vugt.

Claudia Vingerhoets. Investigating neurobiological mechanisms underlying comorbid cognitive symptoms in psychosis and substance use. Supervisors: Prof.dr. T. van Amelsvoort; Prof.dr. J. Booij, UvA; Co-supervisor: Dr. O. Bloemen

Dennis Oerlemans. Evolution of Neuromodulation for Lower Urinary Tract Dysfunction; Past, Present and Future. Supervisors: Prof.dr. Ph. van Kerrebroeck; Prof.dr. G. van Koeveringe. Cosupervisors: Dr. E. Weil; Dr. T. Marcelissen.

Marion Levy. Evaluation of BDNF/TrkB signaling as a common target in the treatment of major depression and Alzheimer's disease. Supervisors: Prof.dr. H. Steinbusch; Prof. L. Lanfumey, Université Paris Descartes, France. Co-supervisors: Dr. G. Kenis; Dr. D. van den Hove. 
Patrick Domen. Stay connected: a family-based diffusion imaging study in psychotic disorder. Supervisor: Prof.dr. J. van Os. Co-supervisor: Dr. M. Marcelis

Geor Bakker. Innovative Approaches to Understanding the Neurobiology of Psychosis. Supervisors: Prof.dr. T. van Amelsfoort; Prof.dr. J. Booij, UvA. Co-supervisor: dr. M. Caan,UvA; dr. O. Bloemen.

Wilma Boevink. HEE! Over Herstel, Empowerment en Ervaringsdeskundigheid in de psychiatrie. Supervisors: Prof.dr. J. van Os; Prof.dr. Ph. Delespaul. Co-supervisor: dr. H. Kroon.

Nataliia Markova . Modified swim test as a mouse depression paradigm of enhanced Cognitive processing: the role of GSK3 $\beta$. Supervisor: Prof.dr. H. Steinbusch; Prof.dr. K-P.Lesch, University of Wuerzburg. Co-supervisor: Dr. T. Strekalova.

Merijn van de Laar. Individual differences in insomnia; implications of Psychological factors for diagnosis and treatment. Supervisor: Prof.dr. A. Aldenkamp; Prof.dr. D. Pevernagie, Universiteit Gent. Co-supervisor: Dr. S. Overeem, TUE.

Willem Buskermolen. If only I could tell ...; Measuring predictors for challenging behaviour in people with both intellectual disability and hearing impairment. Supervisor: Prof.dr. A. Aldenkamp. Co-supervisor: Dr. J. Hoekman, UL.

Kay Deckers. The role of lifestyle factors in primary prevention of dementia; an epidemiological perspective. Supervisor: Prof.dr. F. Verhey. Co-supervisor: Dr. M. vanBoxtel; Dr. S. Köhler.

Brechje Dandachi-FitzGerald. Symptom validity in clinical assessments. Supervisors: Prof.dr. R. Ponds; Prof.dr. F. Verhey.

Maurice Theunissen. Understanding factors affecting postoperative Quality of Life. Supervisors: Prof.dr. M. Peters, Prof.dr. M. Marcus. Co-supervisor: Dr. H. Gramke.

Anna Cleutjens. COgnitive-Pulmonary Disease? Neuropsychological functioning in patients with COPD. Supervisors: Prof.dr. E. Wouters, Prof.dr. R. Ponds. Co-supervisors:Dr. D. Janssen, Horn, Dr. J. Dijkstra.

Laura Serpero. Next Generaton Biomarkers in Perinatal Medicine: S100B Protein. Supervisors: Prof.dr. D. Gazzalo, Alessandria, Italy; Prof.dr. B..W.W. Kramer. Co-supervisor: Dr. A.W.D. Gavilanes. 
Alessandro Varrica. S100B Protein and Congential Heart Diseases: Brain Aspects. Supervisors: Prof.dr. D. Gazzalo, Alessandria, Italy; Prof.dr. J.S.H. Vles; Prof.dr. L.J.I. Zimmermann. Cosupervisor: Dr. A.W.D. Gavilanes.

Pim R.A. Heckman. Targeting phosphodiesterase type 4 for improving cognitive fronto-striatal function: a translational approach. Supervisor: Prof.dr. J.G. Ramaekers. Co- supervisors: Dr. J.H.H.J.. Prickaerts; Dr. A. Blokland.

Sven van Poucke. Platelets, form sample to big data; exploring granularity in platelet research. Supervisors: Prof.dr. M.A.E. Marcus; Prof.dr. W. Buhre. Co-supervisor: Dr. M.Lancé.

Désirée M.J. Vrijens. Dysfunctions of the Lower Urinary Tract and Affective Symptoms. Supervisors: Prof.dr. Ph.E.V. van Kerrebroeck; Prof.dr. G.A. van Koeveringe. Co- supervisors: Dr. C. Leue.

Tamar van Veenendaal. Neurotransmitters \& Networks. An MR view on epilepsy andantiepileptic drugs. Supervisors: Prof.dr.ir. W.H. Backes; Prof.dr. A.P. Aldenkamp. Co- supervisor: Dr. J.F.A. Jansen.

Evelien M. Barendse. Autism Spectrum Disorders in High functioning Adolescents; Diagnostic considerations (AHA). Supervisors: Prof.dr. A.P. Aldenkamp; Prof.dr. R.P.C. Kessels, Radboud University.

Roy Lardenoije. A venture into the epigenetics of aging and Alzheimer's Disease. Supervisors: Prof.dr. B.P.F. Rutten; Prof.dr. H.W.M. Steinbusch. Co-supervisors: Dr. D. van den Hove; Dr. C.A. Lemere, USA.

Charlotte L. Mentzel. The course recognition and treatment of movement disorders in severe mental illness. Supervisors: Prof.dr. P.N. van Harten; Prof.dr. M.A.J. de Koning- Tijssen, UMCG. Co-supervisor: Dr. P.R. Bakker.

Tim Batink. Third Wave Behaviour Therapy: Process Measures and Contextual Interventions. Supervisors: Prof.dr. F.P.M.L. Peeters; Prof.dr. J.J. van Os; Prof.dr. M.C.Wichers, UMC Groningen.

Kevin L.J. Rademakers. Detrusor Underactivity: From Theory To Clinical Assessment. Supervisors: Prof.dr. G.A. van Koeveringe; Prof.dr. Ph.E.V. van Kerrebroeck. Co-supervisor:Dr. M. Oelke.

Iris M.J. Lange. Should I stay or should I go ? Brain mechanisms underlying fear and safety learning, and explosure therapy outcome. Supervisors: Prof.dr. K.R.J. Schruers; Prof.dr. T.A.M.J. van Amelsfoort. Co-supervisor: Dr. L. Goossens. 
Ruben G.F. Hendriksen. Evidence for a dystrophin-associated encephalopathy in Duchenne Muscular Dystrophy. Supervisor: Prof.dr. J.S.H. Vles. Co-supervisors: Dr. G. Hoogland; Dr. M.W. Aalbers, UMC Groningen.

Michael Gofeld. Strengths and limitations of the lumbar spine ultrasound-guided interventions. Supervisor: Prof.dr. M. van Kleef. Co-supervisor: Dr. M. Sommer.

Willem A.R. Zwaans. Strategies for chronic inguinal pain. Supervisor: Prof.dr. M. van Kleef. Cosupervisors: Dr. R.H.M. Roumen; Dr. M.R.M. Scheltinga, MMC Veldhoven.

Linda M. Rolf. Mapping the effects of vitamin D in multiple sclerosis A 3D Perspective. Supervisor: Prof.dr. R.M.M. Hupperts. Co-supervisors: Dr. J.G.M.C. Damoiseaux; Dr. J.J.F.M. Smolders, CWZ Nijmegen.

Maarten van Beek. Spinal Cord Stimulation in Clinical and Experimental Painful Diabetic Polyneuropathy. Supervisors: Prof.dr. E.A. Joosten; Prof.dr. M. van Kleef. Co-supervisor: Dr. S.M.J. van Kuijk.

Melina Barkhuizen. Genetic and perinatal risk factors for movement disorders. Supervisors: prof.dr. B.W.W. Kramer, prof.dr. H.W.M. Steinbusch, Prof.dr. A.F. Grobler. Co- supervisor: dr. A.W.D.Gavilanes-Jimenez.

Renske Uiterwijk. Cognitive function and cerebral small vessel disease in hypertension. Supervisor: prof.dr. R.J. van Oostenbrugge. Co-supervisor: Dr. J.E.A. Staals.

Elles Douven. Depression and apathy after stroke. Supervisor: prof.dr. F.R.J. Verhey. Cosupervisors: Dr. P. Aalten, dr. J. Staals.

Mauro Pessia. Brain K+ Channels: from molecular and physiological features to autismspectrum disorder and intellectual disability. Supervisors: prof.dr. H.W.M. Steinbusch, prof.dr. M.B. Donati, It.

Carsten Leue. Hyperarousal in the Hospital and what to do about it: the MED-PSYCH-NET - a transitional network approach fostering personalized care in psychosomatic medicine. Supervisors: Prof.dr. J. van Os, Prof.dr. A. Masclee. Co-supervisors: Dr. J. Strik, Dr. J. Kruimel

Andrea S. Herrera Soto. Aminochrome, an endotoxin for inducing a new rat model of Parkinson's Disease. Supervisor: prof.dr. H.W.M. Steinbusch. Co-supervisors: Prof.dr. Juan Segura-Aquilar; prof. G. Diaz-Veliz, Santiago of Chile 
Eline E.B. de Clerck. Ocular neurodegenerative changes and macular cysts in prediabetes and type 2 diabetes. Supervisors: Prof.dr. C.A.B. Webers, Prof.dr. C.D.A.Stehouwer. Co-supervisor: Dr. J.S.A.G. Schouten

Steven T.H. Honings. Exploring psychosis and multidirectional violence: a prospectivestudy in the general population. Supervisor: Prof.dr. J. van Os. Co-supervisor: Dr. M. Drukker

\section{8}

Sau May Wong. Advances in Microvasculair MRI Techniques: Breaking the Pathophysiological Barriers in Cerebral Small Vessel Disease. Supervisor: Prof.drir. W.H. Backes, Porf.dr. R.J. van Oostenbrugge. Co-supervisor: Dr. J.F.A. Jansen

Mark B.N. van Winkel. Lonely at heart and stressed in company of Others; the influence of daily life social experiences and emotions on depression. Supervisors: prof.dr. F. Peeters; prof.dr. I. Myin-Germeys, KU Leuven/UM; prof.dr. M. Wichers, UMC Groningen

Harsha Birur Laxmana Rao. Revisiting the vascular theory of glaucoma using optical coherence tomography angiography. Supervisors: prof.dr. C.A.B. Webers; prof.dr. R.N.Weinreb, University of California, San Diego

Babette L.R. Reijs. Cognitive correlates of cerebrospinal fluid biomarkers for Alzheimer's disease. Supervisor: prof.dr. F.R.J. Verhey. Co-supervisors: dr. P.J. Visser;dr. I.H.G.B. Ramakers

Rachel Slangen. Spinal cord stimulation in painful diabetic peripheral Neuropathy. Clinical- and cost-effectiveness. Supervisors: prof.dr. M. van Kleef; Prof.dr. C. Dirksen;prof.dr. C. Faber

Ganne Chaitanya. Epilepsy: A network disorder. Supervisors: prof.dr. A.P. Aldenkamp; prof. P. Satishchandra, NIMHANS, Bangalore, India. Co-supervisors: Dr. J.F.A. Jansen; Dr. S. Zinger, TUE

Sumitha Rajendrarao. New Insight into the Multifaceted Pathogenic Mechanisms of Sporadic Amyotrophic Lateral Sclerosis. Supervisors: prof.dr. B.W. Kamer; prof.dr. H.W. Steinbusch. Cosupervisor: prof. T.R. Raju, NIMHANS, Bangalore, India

Suzanne Roggeveen. Interference of mobile phone with electrophysiology and emotions; results from short-term experimental studies. Supervisor: prof.dr. J. van Os. Co-supervisor: dr. R. Lousberg.

Matthias Walter. Multi-methodological approaches to investigate lower urinary tractfunction in health and disease. Supervisors: prof.dr. Ph.E.V.A. van Kerrebroek; prof.dr. G.A. van Koeveringe; prof.dr. A. Curt, Zürich, $\mathrm{CH}$. 
Lalit Gupta. Inhomogeneities in spontaneous brain fluctuations. Supervisors: prof.dr.ir. WH. Backes; prof.dr. P.A.M. Hofman. Co-supervisor: dr. J.F.A. Jansen.

Chaitra Jayadev. Impact of imaging the pediatric retina. Supervisor: prof.dr. C.A.B. Webers. Cosupervisor: dr. N.J.C. Bauer; dr. A. Vinekar.

Annelie Klippel. Navigating through complexity; processes and mechanisms underlying the development of psychosis. Supervisors: prof.dr. I. Myin-Germeys, KU-Leuven; prof.dr. M.C. Wichers, UMC Groningen. Co-supervisor: dr. U. Reininghaus.

Kürşat Altinbaş. Reconstructing The Diagnostic Framework of Bipolarity. Supervisor:Prof.dr. J. van Os. Co-supervisor: Dr. I.S. Gülöksüz.

Andrea J.R. Balthasar. Eyes of the needle; Spectral tissue sensing, an innovative technology for detecting various tissue types during percutaneous needle-based procedures in locoregional anesthesia and pain medicine. Supervisor: prof.dr. M. van Kleef. Co-supervisor: dr. G-J. van Geffen, Radboud UMC Nijmegen.

Walmari Pilz. Shedding light on oropharyngeal dysphagia in myotonic dystrophy type 1. Supervisor: prof.dr. B. Kremer. Co-supervisors: dr. L.W.J. Baijens; dr. V. Lima Passos.

Nynke J. van den Hoogen. Repetitive painful procedures in the neonate: Treatment andadult pain sensitivity. Supervisors: prof.dr. E.A.J. Joosten, prof.dr. D. Tibboel, Erasmus MC-Sophia, Rotterdam. Co-supervisor: dr. J. Patijn.

Carlota Mestres Gonzalvo. Medication optimisation; Methodological aspects and new strategies. Supervisors: prof.dr. F.R.J. Verhey, prof.dr. P.H.M. van der Kuy, Erasmus MC Rotterdam. Cosupervisors: dr. R. Janknegt, Zuyderland MC.

Carolin Hoffmann. The Brain under Attack: Autoantibodies in Psychotic Disorders. Supervisors: prof.dr. P. Martinez, prof.dr. B. Rutten, prof.dr. J. van Os, UU/UM.

Jindra M. Bakker. On the bumpy road of happiness: Mechanisms of daily life reward processing and how it can be changed. Supervisors: prof.dr. M. Wichers, UMC Groningen, pof.dr. I. MyinGermeys, KU Leuven/UM. Co-supervisor: dr. L. Goossens.

Marasha-Fiona de Jong. Between mood and matter; studies on the interface between mood disorders and physical conditions. Supervisor: prof.dr. F.P.M.L. Peeters. Co- supervisors: prof.dr. Mischoulon. 
Anouk Smeets. New insights in deep brain stimulation for Tourette syndrome. Supervisor: prof.dr. Y. Temel. Co-supervisors: dr. L. Ackermans, dr. A.A. Duits, de. A.F.G.Leentjens.

Margaretha Skowron. Cisplatin resistance in urothelial carcinoma; Understanding and targeting inherent and acquired mechanisms. Supervisors: prof.dr. G.A. van Koeveringe, pof.dr. P. Albers, Heinrich-Heine Univ. Düsseldorf. Co-supervisors: dr. J.G.H. van Roermund, dr. A. Romano.

Thierry Mentzel. Capturing the cacophony of movement. Supervisors: prof.dr. P.N. van Harten, prof.dr. H.A.M. Daanen, VUA. Co-supervisor: dr.mr. O.J.N. Bloemen, GGZ Hilversum/UM.

Petronella de Meij. Quality indicators for the assessment of pain clinic care: A step forward? Quality from professionals and pain patients' perspective (QiPPP). Supervisors: prof.dr. G.D.E.M. van der Weijden, prof.dr. M. v. Kleef. Co-supervisor: dr. A.J.A. Köke.

Thomas Vaessen. Stress sensitivity in psychosis: assessment, mechanism \& intervention. Supervisor: prof.dr. I. Myin-Germeys, KU Leuven/UM.

Yori van der Steen. Dissecting the psychosis continuum; risk factors along the pathway from experiences to disorder. Supervisor: prof.dr. I. Myin-Germeys, KU Leuven/UM, prof.dr. R. van Winkel, KU Leuven.

Aryo Zare. Unveiling the sensory connections between the bladder and the brain thatinvolve the periaqueductal gray matter. Supervisor: prof.dr. G.A. van Koeveringe; Co- supervisor: dr. A. Jahanshahi.

Magdalena Weidner. Brain serotonin throughout development - for better and for worse. Supervisors: prof.dr. H.W.M. Steinbusch, prof.dr. K.P. Lesch, JM.Univ. Würzburg. Co- supervisor: dr. D.L.A. van den Hove.

Catherine Vossen. Cortical processing of pain; the role of habituation. Supervisors: prof.dr. E.A. Joosten, prof.dr. J. van Os, UU/UM. Co-supervisor: dr. R. Lousberg.

Whitney Freeze. Microvascular contributions to dementia; Exploring the role of blood-brain barrier leakage in cerebral small vessel disease and Alzheimer disease. Supervisors: prof.dr. F.R.J. Verhey, prof.dr.ir. W.H. Backes. Co-supervisor: dr. H.I.L. Jacobs.

Simone Schüller. Characterization of Stem and Immune Cell Ontogeny to Inform Prevention and Treatment of Infections in Preterm Newborns. Supervisors: prof.dr. B.W.W. Kramer, prof.dr.med.

A. Berger, Wien. Co-supervisor: dr. E. Villamor. 
Michael J. Kemna. Predicting relapses in ANCA associated vasculitis. Supervisor: prof.dr. J.W. Cohen Tervaert. Co-supervisors: dr. J. Damoiseaux, dr. P. van Paassen.

Artemis latrou. Epigenetics in mental and neurodegenerative disorders. Supervisor: prof.dr. B.P.F. Rutten. Co-supervisors: dr. D.L.A. van den Hove, dr. G. Kenis.

Laura Wielders. Prevention \& Treatment of Cystoid Macular Edema after Cataract Surgery. Supervisor: prof.dr. R.M.M. Nuijts. Co-supervisors: dr. J.S.A.G. Schouten, CWZ Nijmegen, dr. B. Winkens.

Daisy Hoofwijk. The way to understanding Chronic Postsurgical Pain; From clinical and psychological predictors to incorporating genetics. Supervisor: prof.dr. W.F.F.A. Buhre; prof.dr. E.A.J. Joosten; Co-Supervisor: dr. H.-F. Gramke; dr. A.A.A. Fiddelers.

Loes Leenen. Self-management in Epilepsy; The Goal is: "Live with a Z(s)mile. Supervisors: prof.dr. H.J.M. Majoie; prof.dr.mr. S.M.A.A. Evers; prof.dr. C.M. van Heugten.

Chiara Peila. 'Effects of Pasteurization and Refrigerated Storage on Human MilkNeurobiomarkers Concentrations. Supervisors: prof.dr. D. Gazzallo, Alessandria, It./MUMC+; prof.dr. G. Visser, UU; prof.dr. E. Bertino, Alessandria, It.

Raymond van de Berg. The Vestibular Implant: Feasibility in humans. Supervisor: prof.dr. $\mathrm{H}$. Kingma; Co-supervisor: dr. J.-P. Guyot, Université de Genève, $\mathrm{CH}$.

Nils Guinand. The Vestibular Implant: a more stable horizon for patients with a bilateralvestibular deficit? Supervisors: prof.dr. H. Kingma; rof.dr. J.-P. Guyot, Université de Genève, CH.

Jasper Smit. Exploring deep brain stimulation as a treatment for tinnitus. Supervisors: prof.dr. R.J. Stokroos; prof.dr. Y. Temel; Co-supervisor: dr. Jahanshahianvar.

Bindu Paravil Sankaran. Brain MRI in Mitochondrial Disorders: Correlating the Phenotype with Genotype. Supervisor: prof.dr. H. Smeets; prof.dr. A. Taly, NIMHANS,Bangalore, India.

Syenna Schievink. Vascular cognitive impairment; at the heart of the matter. Supervisor: prof.dr. F.R.J. Verhey; prof.dr. R.J. van Oostenbrugge; Co-supervisor: dr. S.Köhler.

Isabelle Bos. Biomarkers of Alzheimer's disease; relations with vascular factors and cognition in the pre-dementia stages. Supervisor: dr. P.J. Visser; prof.dr. F.R.J. Verhey;Co-supervisor: dr. S.J.B. Vos. 
Stijn Michielse. Road work ahead; cerebral pathways mediating Psychological mechanisms underlying the psychosis spectrum. Supervisor: prof.dr. J.J. van Os; Co- supervisor: dr. M.C. Marcelis.

Georgios Schoretsanitis. Risperidone-based therapeutic regimens; Drug interactions andadverse drug reactions. Supervisor: prof.dr. K.R.J. Schruers; Co-supervisor: dr. M. Bak .

Alieske Dam. INLIFE; An innovative online social support intervention for caregivers of persons with dementia. Supervisor: prof.dr. M.E. de Vugt; prof.dr. F.R.J. Verhey; Co- supervisor: dr. M.P.J. van Boxtel.

Roel Haeren. Vascular ventures; Analysis of vascular structures and function in epilepsy. Supervisor: prof.dr. Y Temel; Co-supervisor: dr. K. Rijkers; dr. G. Hoogland.

Chiara Fabbri. Pharmacogenomics of antidepressant drugs: perspectives for thepersonalization of treatment in depression. Supervisors: prof.dr. K. Schruers; prof.dr. A. Serretti, Bologna.

Esther van Duin. Dancing in the (B)rain'; neurobiology of reward, stress \& Information processing in 22q11.2 deletion syndrome. Supervisors: prof.dr. T. van Amelsvoort; prof.dr. J. Booij, UvA. Cosupervisor: dr. D. Hernaus.

Rob Verdonschot. Oropharyngeal dysphagia and its psychiatric Comorbidities; The prevalence of affective symptoms and the unmet clinical need for integrated care in medically unexplained symptoms. Supervisor: prof.dr. B. Kremer; Co-supervisors; dr. L.Baijens; dr. S. Vanbelle.

Lisanne Breuer. Accelerated Cognitive Ageing in Epilepsy' Does it Exists? Supervisors: prof.dr. A. Aldenkamp; prof.dr. P. Boon, UZ Gent; Co-supervisors: dr. A. de Louw, Kempenhaeghe, Heeze; dr.ir. S. Zinger, TUE.

Liselot Kerpershoek. Access to formal dementia care; A European perspective. Supervisors: prof.dr. F. Verhey; prof.dr. M. de Vugt; prof. B. Woods, Bangor University, UKCo-supervisor: dr. C. Wolfs.

Henrietta Steinhart. Same Same but Different; Psychological Interventions and how to Mind the Knowledge Practice Gap. Supervisor: prof.dr. I. Myin-Germeys. Co-supervisor:đr. U. Reininghaus.

Ulrich Mehnert. The management of urine storage dysfunction in the neurological patient. Supervisors: prof.dr. G. van Koeveringe; prof.dr. Ph.van Kerrebroeck; prof.dr. S. Wachter, Antwerpen; prof.dr E. Chartier-Kastler, Sorbonne, Paris. 
Giovanne B. Diniz. Weaning-induced alterations on neuropeptidergic populations of the rat hypothalamus. Supervisors: prof.dr. H. Steinbusch; prof.dr. J. Bittencourt, ICB/USP, Brasil.

Rajani Ravindra Battu. Inherited Retinal Diseases: New Imaging and Molecular Genetics. Supervisor: prof.dr. C.A.B. Webers. Co-supervisors:dr. J.S.A.G. Schouten, CWZ; dr. T.T.J.M. Berendschot.

\section{9}

Jans van Ool. Diagnostic and neuropsychiatric considerations in epilepsy and intellectualdisability; Psychological perspectives. Supervisor: prof.dr. A. Aldenkamp. Co-supervisors: dr. J. Hendriksen; dr. H. Schelhaas, Kempenhaeghe.

Eveline Janssen. Depression in the elderly: focus on high risk groups. Supervisors: prof.dr. F. Verhey; prof.dr. M. de Vugt. Co-supervisor: pr. M. Schram.

Cécile Kicken. Extreme blood coagulation; investigating the influence of physiologicalextremes on thrombin generation and platelet activation. Supervisor: prof.dr. W. BuhreCo-supervisors; dr. B. de Laat; dr. M. Lancé, Qatar.

Martinus van Eerd. Diagnosis and Interventional Pain Treatment of Cervical Facet Joint Pain. Supervisor: prof.dr. M. van Kleef. Co-supervisor; dr. J. Patijn, Eindhoven; dr. M. Sommer.

Chenxing E. Zhang. Novel insights in the pathophysiology of cerebralsmall vessel disease -a study using advanced imaging techniques. Supervisors: prof.dr. R.J. van Oostenbrugge; prof.dr.ir. W.H. Backes; Co-supervisor: dr. J. Staals.

Ivo Eijkenboom. A zebrafish model of small-fiber neuropathy. Supervisors: prof.dr. H.J.M.Smeets; prof.dr. C.G. Faber; Co-supervisor: dr. J. Vanoevelen.

Bianca de Greef. Small fiber neuropathy: from underlying conditions to treatment. Supervisor: prof.dr. C.A. Faber; Co-supervisor: dr. I.S.J. Merkies; dr. J.G.J. Hoeijmakers.

Lotte Berk. MINDFULNESS AND AGING: Exploring Mechanisms and Interventions. Supervisors: prof.dr. J. van Os; prof.dr. M.W. de Vugt; Co-supervisor: dr. M.P.J. van Boxtel.

Mor Dickman. Practice patterns and outcomes of corneal transplantation. Supervisor: prof.dr. R.M.M.A. Nuijts; Co-supervisors: dr. T.J.M. Berendschot; dr. F.J.H.M. van den Biggelaar. 
Thyagi Ponnamperuma. Mental Health Problems in Sri Lankan Adolescents Exposed to the Tsunami and Other Traumatic Events. Supervisor: prof.dr. M.W. De Vries; Co-supervisor: dr. N.A. Nicolson.

Robbert C. Maatman. Anterior cutaneous nerve entrapment syndrome (acnes): an analysis of various subtypes and alternative treatment modalities. Supervisor: prof.dr. M. van Kleef; Cosupervisors: dr. R.M.H. Roumen, dr. M.R.M. Scheltinga.

Mari Elshout. Neovascular Age-Related Macular Degeneration in the Era of Value-Based Health Care. Supervisor: prof.dr. C.A.B. Webers; Co-supervisor: dr. J.S.A.G. Schouten.

Jeroen Deenik. Thinking inside the box; Changing lifestyle to improve the health status of inpatients with severe mental illness. Supervisor: prof.dr. P.N. Harten; Co-supervisors: dr. D.E. Tenback; dr. I.J.M. Hendriksen.

Thomas Draak. Peripheral Neuropathy outcome measures Standardisation (PeriNomS) studypart 3: Capturing the Patient's Voice. Supervisor: prof.dr. C.G. Faber; Co-supervisor: dr. I.S.J. Merkies.

Ana Luisa Gil Martínez. Neuroprotection in neurodegenerative processes associated with Parkinsonism and aging. Correlation between dopaminergic neuronal death and glial activation. Supervisor: prof.dr. H.W.M. Steinbusch, prof.dr. Maria-Trinidad Herrero Ezquerro, University of Murcia.

Bernice J.A. Gulpers. Anxiety in older adults; Correlates, comorbidities and prognosis with lifespan perspectives. Supervisor: prof.dr. F.R.J. Verhey, prof.dr. R.C. Oude Voshaar; Cosupervisor: dr. S. Köhler.

Elke Devocht. Combining a cochlear implant and a hearing aid in opposite ears: Thebest of both worlds. Supervisor: prof.dr. H. Kingma; co- supervisor: dr. E.I.J. George.

Gillian Townend. Rett Syndrome: Recognising the Communication Challenges, Needs and Potential of Individuals Living with a Rare Disease. Supervisor: prof.dr. L.M.G. Curfs; cosupervisor: dr. P.B. Marschik, Med. University of Graz, Austria.

Takashi Koizumi. Genetic and neuroinflammatory components of familial and sporadic cerebral Small Vessel Disease. Supervisor: prof.dr. H. Steinbusch, prof.dr. T. Mizuno,Japan;co-supervisor: dr. S. Foulquier.

Muhammad Ali. Integrative network-based approaches for modelling Human disease.Supervisor: prof.dr. J. Kleinjans; co-supervisor: dr. D. van den Hove; dr. E. Pishva. 
Guillaume Durand. The adaptive side of psychopathy. Investigating adaptive characteristics associated with the psychopathic personality. Supervisor: prof.dr. B. Rutten; co-supervisor: dr J. Lobbestael.

Darius C. Henatsch. Honey: A Novel Treatment in Chronic Ear Infections. Supervisor:prof.dr. R.J. Stokroos; UMC Utrecht/UM; co-supervisor: dr. J.J. Briedé.

Reinhilde J. Melles. Vaginal penetration: pain or pleasure? The role of fear and sexual arousal. Supervisor: prof.dr. M.L. Peters; co-supervisor: dr. M. ter Kuile, LUMC, dr. M. Dewitte.

Raul Felipe Abella Antón. Cardiac Surgery Biochemical Monitoring in Congenital Heart Diseases Infants. Supervisors: prof. dr. D. Gazzolo, prof. dr. L.J.I. Zimmermann, prof. dr. J.S.H. Vles, cosupervisor; dr. A.W.D. Gavilanes.

Francesca M. Snoeijen-Schouwenaars. Diagnostic, neuropsychiatric and therapeutic considerations in epilepsy and intellectual disability - medical perspectives -. Supervisor:prof.dr. A.P. Aldenkamp, co-supervisors: dr. H.J. Schelhaas, SEIN Zwolle; dr. J.G.M. Hendriksen, Kempenhaeghe, Heeze.

Mariëlle H.J. Pruppers. Peripheral Neuropathies: Standardizing Functional Assessment. Supervisors: prof.dr. C.G. Faber; prof.dr. N.C. Notermans, UU; dr. I.S.J. Merkies, ius promovendi.

Shenghua Zong. Autoantibodies in disorders of the brain: expanding the spectrum. Supervisor: prof.dr. P. Marinez; co-supervisor: dr. M. Losen; dr. R. Rouhl.

Jan-Willem Kallewaard. Diagnosis and minimally invasive treatment of chronic discogenic low back pain. Supervisor: prof.dr. M. van Kleef; co-supervisors: prof.dr. H. van Santbrink; dr. P. Willems.

Simone M. Crivelli. Sphingolipid metabolism in the pathophysiology and treatment of Alzheimer's disease. Supervisors: prof.dr. P. Martinez-Martinez; prof.dr. E. deVries, VUmc. Co-supervisors: dr. M. Losen; dr. M. Mulder, Rotterdam.

Natasha Pahuja. Etiopathogenesis, advanced imaging and treatment outcomes in Asian Indians with keratoconus. Supervisor: prof.dr. R. Nuijts, co-supervisor: dr. R.Shetty, Bengaluru.

Pooja Khamar Mayur Raksha. Clinical, Molecular and Biomechanical outcomes of SMILE (small incision lenticule extraction) and other refractive surgery techniques. Supervisor: prof.dr. R. Nuijts, co-supervisor: dr. R. Shetty, Bengaluru. 
Niels Janssen. Patterns and pathways. Indicators for potential improvements of dementia care. Supervisors: prof.dr. F. Verhey; prof.dr.mr. S. Evers; Co-supervisor: dr. R.Handels.

Giovanni Mansueto. Childhood adversities and Psychosis: investigation of the potential aetiopathogenetic mechanisms. Supervisor: prof.dr. K. Schruers; co-supervisors: prof.dr. F. Cosci, University of Florence, It; prof.dr. R. van Winkel, KU Leuven.

Joke Debruyne. Cochlear implantation in adults with early-onset deafness. Supervisors:prof.dr. B. Kremer; prof.dr.ir. T. Francart, KU Leuven; Co-supervisor: dr.ir. J. Brokx.

Koenraad Meuwissen, Burst Spinal Cord Stimulation in a Rat Model of Chronic Neuropathic Pain: Spinal and Supraspinal Mechanisms. Supervisors: prof. dr. E.A.J.Joosten; prof. dr. M. van Kleef.

Lisa Schmiedek, Episodic memory in ageing and AD: a possible target for electrical stimulation ? Supervisors: prof. dr. F.R.J. Verhey; prof. dr. A.T. Sack; co-supervisor: dr. H.I.L. Jacobs

Paolo Maino, Implantable Intrathecal Drug Delivery in Treatment of Chronic Intractable Pain and Spasticity: Improvement of Safety and the Use of Imaging Techniques. Supervisors: prof. dr. E.A. Joosten; prof. dr. M. van Kleef.

José Geurts, Chronic Pain; Impact of Chronic Pain on a Societal, Personal, and Treatment Level. Supervisors: prof. dr. C.D. Dirksen; prof.dr. M. van Kleef; co-supervisor:dr. P.C. Willems.

Brigitte Brouwer, Painful Small Fiber Neuropathy; Symptoms, assessments and interventions. Supervisor: prof. dr. C.F. Faber; co-supervisors: dr. I.S.J. Merkies, Willemstad, Curaçao; dr. J.G.J. Hoeijmakers.

Ruth Gussenhoven, Antenatal inflammatory insults and preterm brain injury: Pathophysiology and therapeutic strategies. Supervisors: prof. dr. B.W. Kramer; prof. dr. L.J.I. Zimmermann; dr. T.G.A.M. Wolffs.

Adriana (Janine) Collet, Specific Care on the Interface of Mental health and Nursing home "SpeCIMeN". Supervisors: prof. dr. M.E. de Vugt; prof. dr. J.M.G.A. Schols; pProf. dr. F.R.J. Verhey.

Fares Nigim, Glioblastoma and Meningioma Biology, Targeted Therapy and Oncolytic Virus Therapy. Supervisors: prof. dr. Y. Temel; prof. dr. S.D. Rabkin, Harvard; co- supervisors: dr. H. Wakimoto, Harvard; dr. L. Ackermans.

Leonie Banning, Neuropsychiatric symptoms in Alzheimer's disease; Associations withbiomarkers. Supervisor: prof. dr. F.R.J. Verhey; co-supervisors: dr. P. Aalten; dr. I.H.G.B.Ramakers. 
Johan Haumann, Prevalence and pharmacological treatment of pain in patients with cancer; The role of opioids with and without NMDA receptor affinity. Supervisor: prof.dr. E.A. Joosten; cosupervisors: prof.dr. M.H.J. van den Beuken-van Everdingen; dr. S.M.J. Van Kuijk.

Joost Riphagen, Vascular matters in aging and dementia. Supervisor: prof.dr. F.R.J.Verhey; cosupervisor: dr. H.I.L. Jacobs.

Nikos Priovoulos, Structural and functional imaging of the locus coeruleus at 7T: from methodological to clinical application. Supervisor: prof.dr. F.R.J. Verhey; co-supervisors:dr. H.I.L. Jacobs; dr. B.A. Poser.

Simone Verhagen, The power of individual landscapes; A clinical exploration of personal experience sampling and new horizons. Supervisors: prof.dr. P.A.E.G. Delespaul; prof.dr. J.J. van Os, UM/UU; co-supervisor: dr. C.J.P. Simons.

Nagy Youssef, Epigenetics, resilience and brain stimulation: advances in the mechanistic and therapeutic utility in patients with affective (PTSD and mood) disorders. Supervisor:prof.dr. B.P.F. Rutten; co-supervisor: prof. dr. P. Sienaert, KU Leuven.

Abhishek Appaji, Retinal vascular features as a biomarker for psychiatric disorders. Supervisor: prof. dr. C.A.B. Webers; co-supervisor: dr. T.T.J.M. Berendschot, dr. Naren P.Rao.

Koos Hovinga, Angiogenesis Inhibition in Glioblastoma. Supervisor: prof. dr. Y. Temel; cosupervisor: Prof. V. Tabar, New York, USA.

Gerhard Drenthen, Myelin and networks, Magnetic Resonance Imaging in Epilepsy. Supervisors: prof.dr.ir. W.H. Backes; prof.dr. A.P. Aldenkamp; co-supervisor: dr. J.F.A.Jansen.

Anna Gorlova, Understanding the Molecular Mechanisms of Aggression in BALB/C and TPH2Deficient Mice. Supervisor: prof.dr. K. Lesch, Universitätsklinikum Würzburg, co- supervisors: dr. T. Strekalova; prof.dr. L. Bettendorff, University of Liège.

Ekaterina Veniaminova, The impact of the 'Western Diet' on Emotional, Social and Cognitive Behaviours as revealed by a study on conventional and serotonin Transporter-Deficient Mice. Supervisor: prof.dr. K. Lesch, Universitätsklinikum Würzburg, co-supervisors: dr. T. Strekalova; prof. D.C. Anthony, Oxford.

Dmitrii Pavlov, The contribution of CNS inflammation and Glycogen Synthase Kinase-3 (GSK-3)cascades on adverse memory learning on mouse models of emotional stress. Supervisor: prof.dr. K. Lesch, Universitätsklinikum Würzburg, co-supervisors: dr. T.Strekalova; prof.dr. L. Bettendorff, University of Liège. 
Eric Fonseca Wald, Absence Epilepsy and Panayiotopoulos Synrome: Neurocognition and Brain Development. Supervisor: prof.dr. R.J. Vermeulen; co-supervisors: dr. S. Klinkenberg; dr. M.J.A. Debeij-van Hall; dr. J.G.M. Hendriksen, Epilepsiecentrum Kempenhaeghe.

Kimberley S. Noij, Cervical vestibular evoked myogenic potentials; Toward optimizing clinical use. Supervisors: prof.dr. H. Kingma; prof. S.D. Rauch, MD, Massachusetts Eye and Ear, Harvard; cosupervisor: dr. R. van de Berg.

Mark J. van Tilburg, Advancement in CVEMP's. Supervisors: prof.dr. H. Kingma; prof.dr. S. Rauch, Harvard; co-supervisors: dr. R. van de Berg; dr. B. Herrmann, Boston.

Nalini Atcharayam, Duchenne Muscular Dystrophy: The NIMHANS Experience. Supervisors: prof.dr. T. Delhaas; prof.dr. B.W. Kramer.

Murat L Atagün, Cognitive neurophsysiology and neurochemistry in bipolar disorder. Supervisor: prof. dr. Therese van Amelsvoort; co-supervisors: dr. Sinan Guloksuz; dr. Marian Drukker.

Majed Aldehri, Deep brain stimulation, memory functions and mechanisms. Supervisor: prof. dr. Y. Temel; co-supervisors: dr. S. Hescham; dr. A. Jahanshahianvar.

Printha Kentheeswaran-Wijesinghe, Age-related cytoskeletal pathologies: A study on elderly brains to investigate the extent of neuropathological and cerebrovascular changes at death ad their risk factors. Supervisor: prof. dr. H. Steinbusch, prof. dr. R. De Silva - (University of Sri Jayewardenepura), prof. dr. D. Shankar - (NIMHANS Bangalore).

Mahmoud Elbatrik, Network pharmacology for mechanistically redefined comorbidities. Supervisor: prof. dr. H.H.H.W. Schmidt; co-supervisors: dr. A.I. Casas Guijarro.

Alexander Grønning, Big Data Analytics in Bioinformatics. Supervisors: prof. dr. J. Baumbach (University of Southern, Denmark), prof. dr. H.H.H.W. Schmidt; co-supervisor:dr. R. Röttger.

Britta Nijsse, Cognition after stroke; various perspectives. Supervisors: prof. dr. C.M. vanHeugten, prof. dr. J.M.A. Visser/Meily, prof. dr. J.M. Spikman; co-supervisor: dr. P.L.M. deKort.

Eva Koetsier. Dorsal Root Ganglion Stimulation for Pain Relief in Painful Polyneuropathy: Efficacy and Mechanism of Action. Supervisors: prof. dr. E.A.J Joosten,prof. dr. J.A.M. van Zundert; cosupervisor: dr. S.M.J. van Kuijk.

Youssef Yakkioui, Molecular biomarkers in skull base chordoma. Supervisors: prof. dr. Y.Temel, prof. dr. M. van Engeland. 
Sascha Meyer, Visual Associative Learning in Alzheimer's Disease and Performance Validity. Supervisor: prof. dr. R.W.H.M. Ponds; co-supervisor: dr. J.F.M. de Jonghe.

Daniël Verberne, Psychosocial outcome after stroke and traumatic brain injury - Longitudinal perspectives and recommendations for aftercare. Supervisors: prof. dr. C.M. van Heugten, prof. dr. R.W.H.M. Ponds; co-supervisor: dr. M.E.A.L. Kroese.

Britt van Hagen, Improving Pattern Separation and Cognition: Effects of Pharmacological Interventions on Rodent Behavior and Neuroplasticity. Supervisors: prof. dr. J. Prickaerts, prof. dr. H. Schmidt.

Sara Bartels, Monitoring Everyday Life in Aging \& Dementia - Perspectives from Experience Sampling and Technology Use. Supervisors: prof. dr. F.R.J. Verhey, prof. dr. M.E. de Vugt; cosupervisors: dr. R.J.M. van Knippenberg, dr. C. Malinowsky -(Karolinska Institutet, Sweden).

Roel van Reij, Genetic Risk Factors in prediction and tratment of Chronic Post-Surgical Pain. Supervisor: prof. dr. E.A.J. Joosten; co-supervisor: dr. N.J. van den Hoogen.

Hannah Christie, The Implementation of EHealth in Dementia Care: Lessons Learned.Supervisors: prof. dr. M.E. de Vugt, prof. dr. F.R.J. Verhey; co-supervisor: dr. H.J. Tange.

Antoine Bernas, Resting-state fMRI neurodynamics in neuropsychiatric disorders. Supervisors: prof.dr. A.P. Aldenkamp dr. ir. S. Zinger, TUE.

Gwendoline Montes Diaz, Immune regulation by mimethyl fumarate (DMF) in relapsing-remitting multiple sclerosis patients. Supervisors: prof.dr. R. Hupperts, prof.dr. V. Somers, Hasselt, dr. J. Fraussen, Hasselt.

Anne Mulders, Monitoring and Modulating Neuropsychiatric Symptoms in Parkinson's disease and obsessive-compulsive disorder. Supervisors: prof.dr. A.F.G. Leentjens, prof.dr. Y. Temel.

Mayke Oosterloo, Controversies and pitfalls in diagnosing Huntington's Disease. Supervisors: prof. dr. R.A.C. Roos (LUMC, Leiden), prof. dr. C.E.M. de Die-Smulders; co-supervisor: dr. E.K. Bijlsma (LUMC, Leiden).

Franciscus Bennis, Machine learning in medicine - Big picture require small, but crucialstrokes. Supervisors: prof.dr. T. Delhaas, prof.dr. B.W. Kramer; co-supervisor: dr. P. Andriessen (Máxima Medisch Centrum Veldhoven). 
Umut Kirli, Exploring Psychotic Experiences in the Context of Multidimensional Psychopathology: A Longitudinal Community-based Approach. Supervisor: prof.dr. J.van Os; co-supervisors: dr. M. Drukker, dr. T. Binbay, Dokuz Eylul University, Turkey.

Ricky Kamps, Resolving the Role of Genetic Defects and mtDNA Copy Number in Mitochondrial Disease and Development. Supervisor: prof. dr. H.J.M. Smeets; co- supervisor: dr. F.H.J. van Tienen.

Juliette Hoevenaars, Knowledge, adherence and outcome in glaucoma. Supervisor: prof.dr. C.A.B. Webers; co-supervisor: dr. J.S.A.G. Schouten, CWZ, Nijmegen.

Glenn Franken, Neuromodulation of the Dorsal Root Ganglion in Experimental Chronic Neuropathic Pain: Efficacy and Mechanisms of Action. Supervisors: prof. dr. E.A.J. Joosten, prof. dr. J.H.M. van Zundert; co-supervisors: dr. A.L. Liem, MC Jan van Goyen, Amsterdam.

Raghu Raghu, Automated seizure detection for remote monitoring. Supervisors: prof. dr. Y. Temel, prof. dr. N. Sriraam, Karnataka, India; co-supervisors: dr. P.L. Kubben, dr. E.D.Gommer.

Jonas Weijers, Mentalization and psychosis Trying to understand the "un- understandable". Supervisors: prof. dr. J.P.C.J. Selten, prof. dr. E.H.M. Eurelings-Bontekoe, Universiteit Leiden; cosupervisor: dr. W. Viechtbauer.

Jan Wouter Brunings, Concerning Assumptions in Laryngology. Supervisor: prof. dr. B.Kremer; cosupervisors: dr. L.W.J. Baijens, dr. A.E.W. Hamaekers.

Suzanne van Bronswijk, Personalized treatment strategies for depression. Supervisors: prof. dr. F.P.M.L. Peeters, prof. dr. M.J.H. Huibers, VU Amsterdam; co-supervisor: dr. L.H.J.M. Lemmens.

Lotta-Katrin Pries, The interplay between the genome and the exposome in psychosisspectrum. Supervisors: prof. dr. B.P.F. Rutten, prof. dr. J. van Os; co-supervisor: dr. S. Guloksuz.

Suat Kucukgoncu, Metabolic Disturbances in Mental Illness. Supervisor: prof. dr. B.P.F.Rutten; cosupervisor: dr. S. Gölöksüz.

Raffael Pazinatto Aguiar, Synthesis and biological evaluation of new tyrosol-salicylatederivatives as potential antiinflammatory agents. Supervisor: prof dr. J. Prickaerts; co-supervisor: dr Rúbia Maria Weffort de Oliveira, State University of Maringá Maringá, Paraná, Brazil.

Marina Damas, Autoantibodies in the nervous system: pathophysiology and new therapeutic strategies. Supervisor: prof. dr. P. Martinez; co-supervisors: dr. M. Losen, dr. R.P.W. Rouhl. 
Angélique Gruters, Neuropsychological Assessment at the Memory Clinic: Innovations in communicating neuropsychological test results in the diagnostics of dementia. Supervisor: prof. dr. V.C.G. Tjan-Heijnen; co-supervisors: dr. LE.M. Duijm, CanisiusNijmegen, dr. A.C. Voogd.

Clara Snijders, Post-traumatic stress disorder epigenetic signatures of differential susceptibility to combat trauma. Supervisor: prof. dr. B.P.F. Rutten; co-supervisors: dr. L. de Nijs, dr. G. Kenis.

Raoul Stevens, Unboxing the Brain; Development of Technologies for Non- Invasive Assessment of Cerebral Pathologies. Supervisor: prof. dr. T. Delhaas, prof.dDr. W.H. Mess; co-supervisors: dr.ir. W. Huberts.

Talakad Narasappa Sathyaprabha, Cardiovascular autonomic regulation in health and neurological disorders. Supervisors: prof.dr. B.W.W. Kramer, prof.dr. H.W.M. Steinbusch; cosupervisors: dr. T.R. Raju, Bangalore. 
